\title{
Building \\ a National \\ Culture of Health
}

\author{
BACKGROUND, \\ ACTION FRAMEWORK, \\ MEASURES, \\ $A N D$ \\ NEXT STEPS
}

Anita Chandra, Joie D. Acosta, Katherine Grace Carman, Tamara Dubowitz, Laura Leviton, Laurie T. Martin, Carolyn Miller, Christopher Nelson, Tracy Orleans, Margaret Tait, Matthew D. Trujillo, Vivian L. Towe, Douglas Yeung, Alonzo L. Plough 
For more information on this publication, visit www.rand.org/t/rr1199

Library of Congress Cataloging-in-Publication Data

is available for this publication.

ISBN: 978-0-8330-9294-6

Published by the RAND Corporation, Santa Monica, Calif.

(C) Copyright 2016 RAND Corporation

RAND $^{\circledR}$ is a registered trademark.

\section{Limited Print and Electronic Distribution Rights}

This document and trademark(s) contained herein are protected by law. This representation of RAND intellectual property is provided for noncommercial use only. Unauthorized posting of this publication online is prohibited. Permission is given to duplicate this document for personal use only, as long as it is unaltered and complete. Permission is required from RAND to reproduce, or reuse in another form, any of its research documents for commercial use. For information on reprint and linking permissions, please visit www.rand.org/pubs/permissions.html.

The RAND Corporation is a research organization that develops solutions to public policy challenges to help make communities throughout the world safer and more secure, healthier and more prosperous. RAND is nonprofit, nonpartisan, and committed to the public interest.

RAND's publications do not necessarily reflect the opinions of its research clients and sponsors.

Support RAND

Make a tax-deductible charitable contribution at www.rand.org/giving/contribute

www.rand.org 
Since 2013, the Robert Wood Johnson Foundation (RWJF) has embarked on a pioneering effort to advance a Culture of Health. The Culture of Health action framework is founded on a vision in which "everyone in our diverse society leads healthier lives now and for generations to come." To put this vision into action, RWJF asked the RAND Corporation to support the development of an action framework and measurement strategy. This report provides background on the development of this action framework, with attention to action areas and drivers. Action areas are the core areas in which investment and activity are needed to activate and cultivate a Culture of Health broadly, while drivers indicate where the nation needs to accelerate change within those action areas specifically. The report also documents the first phase in developing the Culture of Health measurement strategy and serves as a complement to other reports, including the RWJF publication From Vision to Action: A Framework and Measures to Mobilize a Culture of Health (Plough et al., 2015) and the RAND report Stakeholder Perspectives on a Culture of Health: Key Findings (Acosta et al., 2015). The report draws on an environmental scan, diverse stakeholder engagement, and a series of inputs from core RWJF partners. Additional information about the Culture of Health, including detail on measures, can be found at www.cultureofhealth.org.

This research was conducted jointly by researchers from RAND and RWJF and is intended for individuals and organizations interested in understanding and advancing the Culture of Health action framework. Given that RWJF is focused on using the action framework and measures to catalyze a national dialogue about content and investments to improve population health and well-being, the report should be beneficial to a range of national, state, and local leaders across a variety of sectors that contribute to health.

This research was sponsored by the Robert Wood Johnson Foundation and conducted within RAND Health. A profile of RAND Health, abstracts of its publications, and ordering information can be found at www.rand.org/health. 


\section{Contents}

Preface

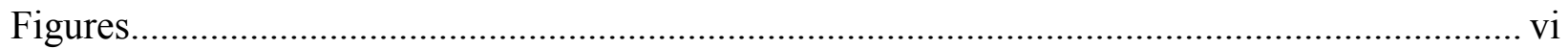

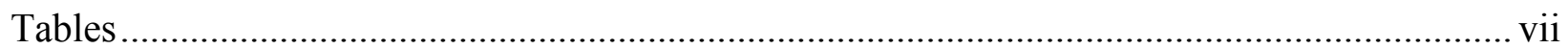

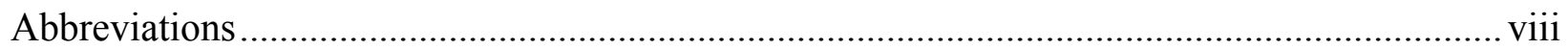

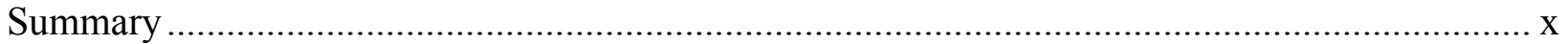

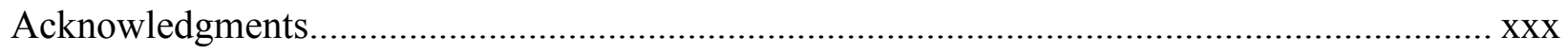

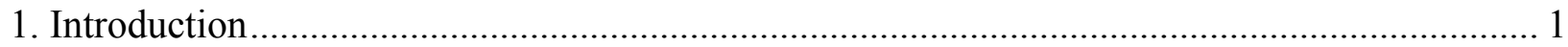

Defining Culture

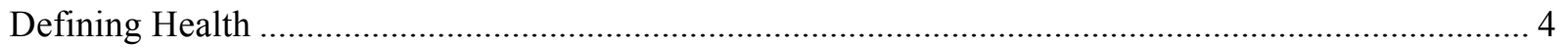

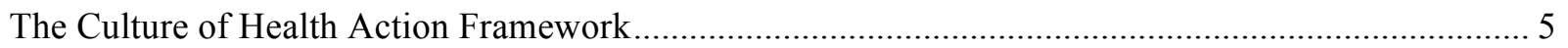

Methods Employed for Action Framework Development …....................................................... 7

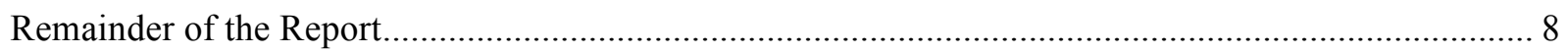

2. Developing the Action Framework and Drivers .......................................................... 10

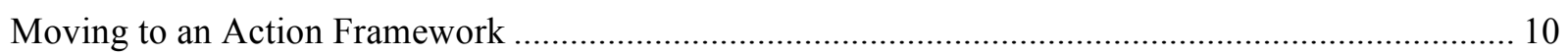

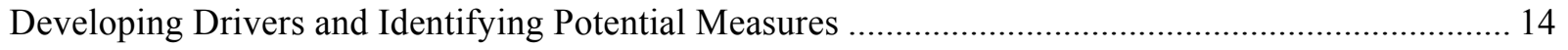

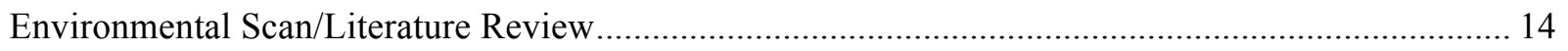

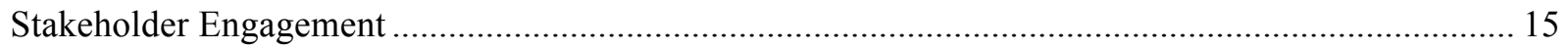

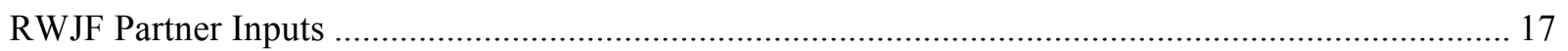

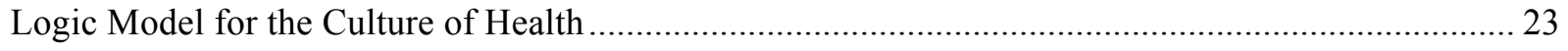

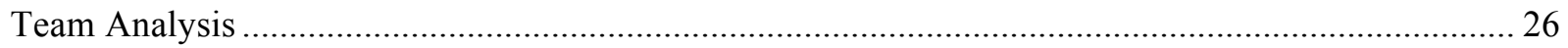

3. Toward a Strategy for Measuring a Culture of Health .................................................. 27

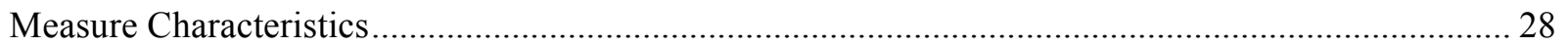

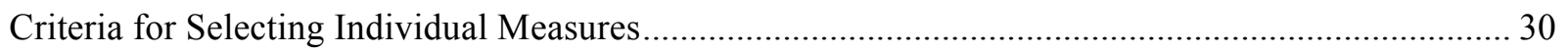

4. Stakeholder Engagement to Inform the Culture of Health Action Framework...................... 37

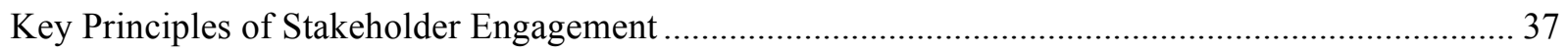

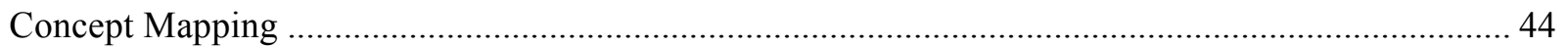

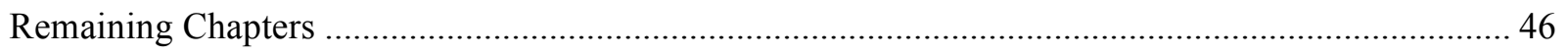

5. Making Health a Shared Value ................................................................................. 48

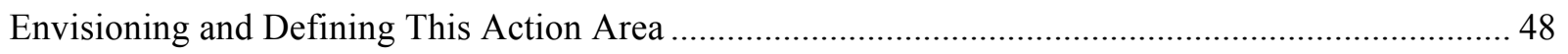

Drivers in the Action Area of Making Health a Shared Value........................................................... 49

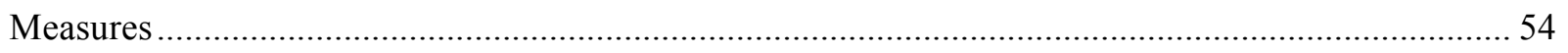

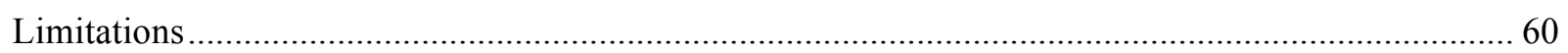

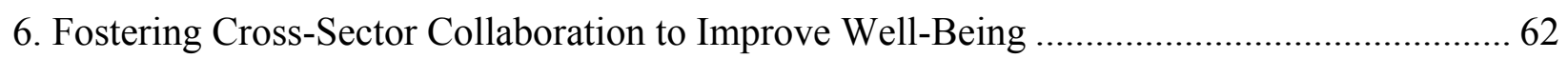

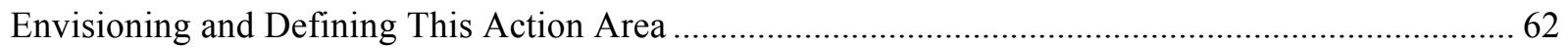

Drivers in the Action Area of Fostering Cross-Sector Collaboration to Improve Well-Being .............. 64

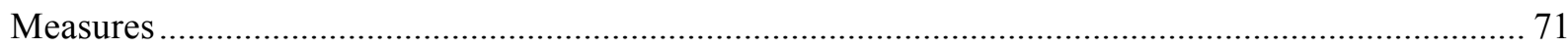

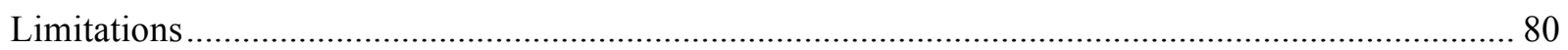


7. Creating Healthier, More Equitable Communities ........................................................... 82

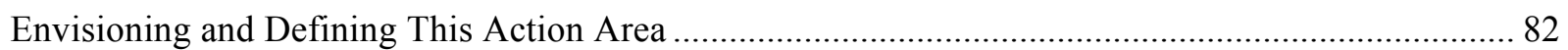

Drivers in the Action Area of Creating Healthier, More Equitable Communities ................................ 84

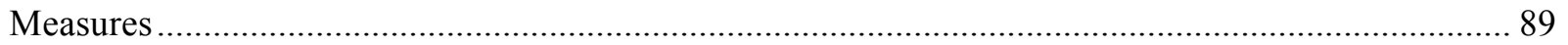

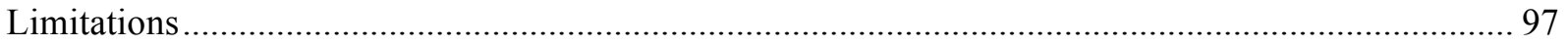

8. Strengthening Integration of Health Services and Systems .............................................. 99

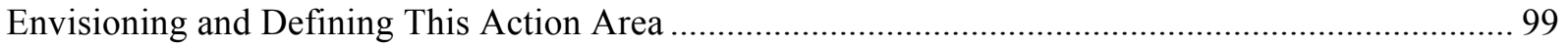

Drivers in the Action Area of Strengthening Integration of Health Services and Systems................. 102

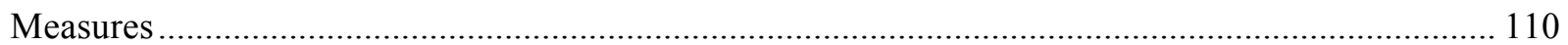

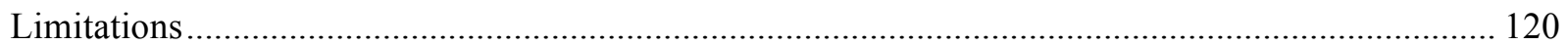

9. Improving Population Health, Well-Being, and Equity................................................. 122

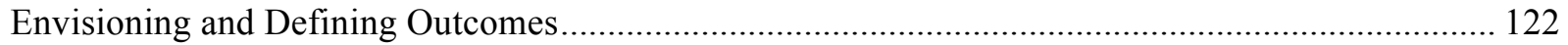

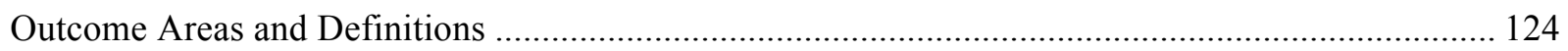

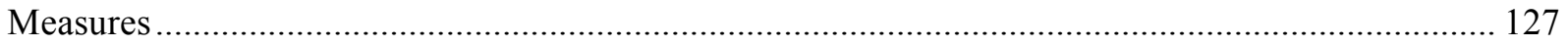

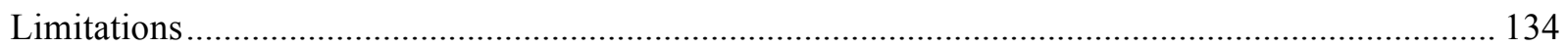

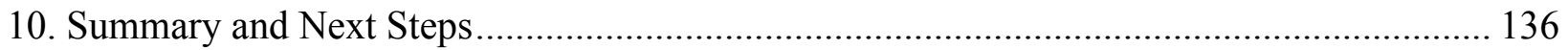

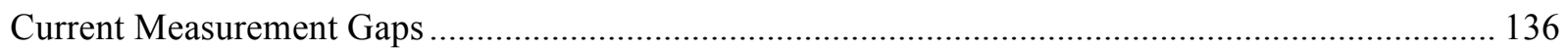

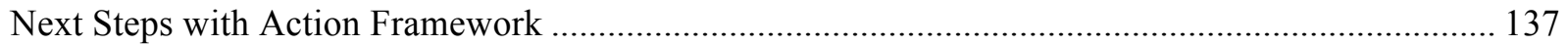

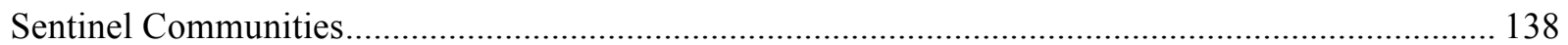

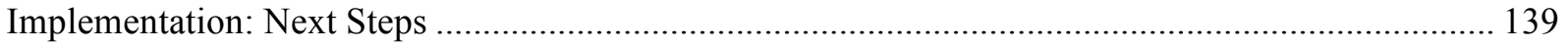

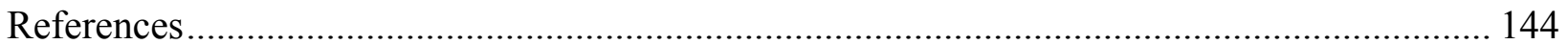

Appendix A. Frequently Asked Questions About the Culture of Health ............................... 170

Appendix B. Sentinel Community Overview and Frequently Asked Questions...................... 178 
Figures

Figure S.1. Culture of Health Action Framework....................................................................iii

Figure S.2. Process for Action Framework Development ....................................................... xiv

Figure 1.1. Culture of Health Action Framework ............................................................. 6

Figure 1.2. RWJF and RAND Collaboration to Develop Action Framework............................. 7

Figure 1.3. Process for Action Framework Development ..................................................... 8

Figure 2.1. Logic Model for Assessing Culture of Health Progress ....................................... 25

Figure 3.1. Reviewing Culture of Health Measures .............................................................. 32

Figure 4.1. Culture of Health Pilot Concept Map ................................................................. 46

Figure 10.1. Learning Process for Culture of Health Measures ........................................... 141 
Table 2.1. Map of Culture of Health Principles to Action Framework Action Areas 11

Table 2.2. HERO Core Elements of Culture That Are Critical for Establishing a Culture of

Health in the Workplace ......................................................................................... 20

Table 3.1. Characteristics of Culture of Health Measures ...................................................... 29

Table 3.2. Primary Measures Review Criteria..................................................................... 30

Table 3.3. Measures by Action and Outcome Area ........................................................... 31

Table 4.1. Community Approaches to Building a Culture of Health ....................................... 42

Table 5.1. Factors Influencing Mindset and Expectations for Health .................................... 51

Table 5.2. Factors Influencing Sense of Community ........................................................... 52

Table 5.3. Factors Influencing Civic Engagement............................................................ 54

Table 5.4. Making Health a Shared Value: Measures by Driver ............................................... 59

Table 6.1. Factors Influencing Partnership Development and Quality.................................... 68

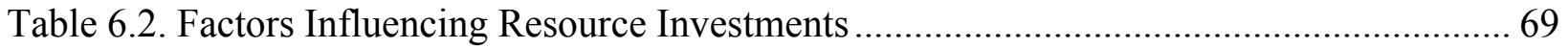

Table 6.3. Fostering Cross-Sector Collaboration to Improve Well-Being: Measures

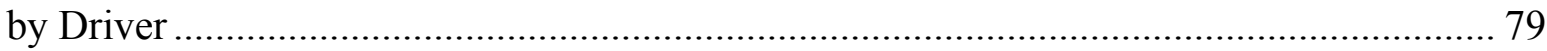

Table 7.1. Factors Influencing the Built Environment and Physical Conditions....................... 86

Table 7.2. Factors Influencing the Social and Economic Environment ................................. 87

Table 7.3. Factors Influencing Policy and Governance...................................................... 89

Table 7.4. Creating Healthier, More Equitable Communities: Measures by Driver .................. 96

Table 8.1. Factors Influencing Access .................................................................................. 104

Table 8.2. Factors Influencing Consumer Experience and Quality ....................................... 107

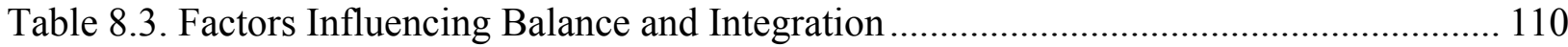

Table 8.4. Strengthening Integration of Health Services and Systems: Measures by Driver.... 119

Table 9.1. Population Health, Well-Being, and Equity: Measures by Outcome Area .............. 133 
ABCD asset-based community development

ACA

Affordable Care Act

$\mathrm{ACE}$

adverse child experience

$\mathrm{ACO}$

accountable care organization

AHRQ

Agency for Healthcare Research and Quality

BLI

Better Life Index

BMI

body mass index

BRFSS Behavioral Risk Factor Surveillance System

CAHPS Consumer Assessment of Healthcare Providers and Systems

$\mathrm{CDC}$

Centers for Disease Control and Prevention

CECP

Committee Encouraging Corporate Philanthropy

CFBAI Children's Food and Beverage Advertising Initiative

CHIP community health improvement plan

CHNA community health needs assessment

CHR County Health Ranking

DALY disability-adjusted life year

EHR electronic health record

EMR electronic medical record

FMLA Family and Medical Leave Act

HERO Health Enhancement Research Organization

HHS U.S. Department of Health and Human Services

HIT health information technology

HRET Health Research \& Educational Trust

HRSA Health Resources and Services Administration

IHME Institute for Health Metrics and Evaluation

LHD local health department

NACCHO National Association of County and City Health Officials

NEA

National Education Association

NIEER National Institute for Early Education Research

NP nurse practitioner

NRDC Natural Resources Defense Council

NSDUH National Survey on Drug Use and Health 
OECD Organisation for Economic Co-operation and Development

PCMH patient-centered medical home

RWJF Robert Wood Johnson Foundation

SAMHSA Substance Abuse and Mental Health Services Administration

SBHC school-based health center

$\mathrm{SCI}$

Sense of Community Index

SNAP Supplemental Nutrition Assistance Program

SUPPORT Study to Understand Prognosis and Preferences for Outcomes and Risks of Treatment

USDA U.S. Department of Agriculture

WHO World Health Organization

WIC Women, Infants, and Children 


\section{Summary}

Since 2013, the Robert Wood Johnson Foundation (RWJF) has embarked on a pioneering effort to advance a Culture of Health, framed by ten core principles. RWJF asked the RAND Corporation to support the Foundation's development of an action framework and measurement strategy for a Culture of Health, building on these ten core principles. This report provides background on the development of this action framework, particularly the drivers and illustrative measures. The report also documents the first phase in developing the Culture of Health measurement strategy and complements other reports, including the RWJF publication From Vision to Action: A Framework and Measures to Mobilize a Culture of Health (Plough et al., 2015) and the RAND report Stakeholder Perspectives on a Culture of Health: Key Findings (Acosta et al., 2015). It draws on an environmental scan, diverse stakeholder engagement, and a series of inputs from core RWJF partners (e.g., analyses on health equity by Prevention Institute, business roles in workplace health by Health Enhancement Research Organization [HERO]).

\section{Initial Motivation for the Culture of Health}

Health and health care are at a crossroads in the United States (Davis et al., 2014; OECD, 2013). The country spends more on health care than any other nation. But Americans are not getting their money's worth. For example, the United States ranks only 26th in life expectancy among 40 of the most developed countries (Bauer et al., 2014; Galea et al., 2011; Link and Phelan, 1995; Mokdad et al., 2004; OECD, 2013). It has become increasingly clear that solutions to U.S. health problems must encompass more than reforms to health care systems. Health is a function of more than medical care. Health behaviors and the social and physical environment in which individuals live and work have a stronger influence on well-being than clinical services alone (Braveman, 2014; Galea et al., 2011).

This emerging understanding and the changing landscape of our nation's health suggest a need to rethink the relationships among all sectors of activity and their roles in health. This includes reimagining the health sector as one that balances health, public health, and social service systems to yield the highest value to individuals and communities. Some of this change in orientation to a broader view of health has been required as demographics shift (e.g., 
population aging, increase in immigration) (Pol and Thomas, 2000; U.S. Census Bureau, 2010). Coordinated efforts to promote wellness and prevent diseases are proliferating among a diverse set of stakeholders, including organizations that have not traditionally focused on health (Galea et al., 2011; Link and Phelan, 1995; Mokdad et al., 2004; OECD, 2013; University of Wisconsin Population Health Institute, 2014). These shifts have helped catalyze a national movement that demands and supports a widely shared, multifaceted vision for a Culture of Health.

In this context, RWJF laid out the ten core principles for a Culture of Health, initially delineated in Building a Culture of Health (Lavizzo-Mourey, 2014):

1. Optimal health and well-being flourish across geographic, demographic, and social sectors.

2. Opportunities to be healthy and stay healthy are valued and accessible to everyone across the entire society.

3. Individuals and families have the means and opportunities to make choices that lead to healthy lifestyles and optimal well-being and functioning.

4. Business, government, individuals, and organizations work together to foster healthy communities and lifestyles.

5. Everyone has access to affordable, high-quality health care - both preventive and remedial.

6. No one is excluded.

7. Health care is efficient and equitable.

8. The economy is less burdened by excessive and unwarranted health care spending.

9. The health of the population guides public and private decisionmaking.

10. Americans understand that we are all in this together.

\section{Development of the Action Framework}

While the ten guiding RWJF principles provided the critical foundation for describing what constitutes the end state of a Culture of Health, RWJF did not yet provide the operational or action framework for how the nation could begin to work toward achieving these outcomes. Since late 2013, RWJF has worked collaboratively with RAND to develop an action framework for the Culture of Health. The ten principles underlying the Culture of Health vision created a starting point for this action framework. 
The goal of the framework (Figure S.1) is to convey a holistic, integrated perspective on what it takes to achieve population-level health and well-being. The Culture of Health action framework is designed around four action areas and one outcome area. The action areas include (1) making health a shared value; (2) fostering cross-sector collaboration to improve well-being; (3) creating healthier, more equitable communities; and (4) strengthening integration of health services and systems. The outcome area —improved population health, well-being, and equityis intended to serve as the key result of activities in each action area. While not represented in the figure, each action area contains a set of drivers indicating where the United States needs to accelerate change. The drivers provide a set of investment priorities that are core to sustained improvement in health over time and maintained progress in the action area, and, thus, they do not change over time. Each action area is also accompanied by a set of measures to illustrate progress in an action area. These measures are meant to invite dialogue among stakeholders to discuss key areas for progress in the action area (e.g., a measure on library access not only engages this sector but starts conversations about well-being amenities in communities) but are not intended to holistically capture all of the aspects to measure in a given action area. In other words, the measures serve as a starting point, but we recognize that various stakeholder groups may identify other aspects that merit monitoring or tracking for a given action area. 
Figure S.1. Culture of Health Action Framework

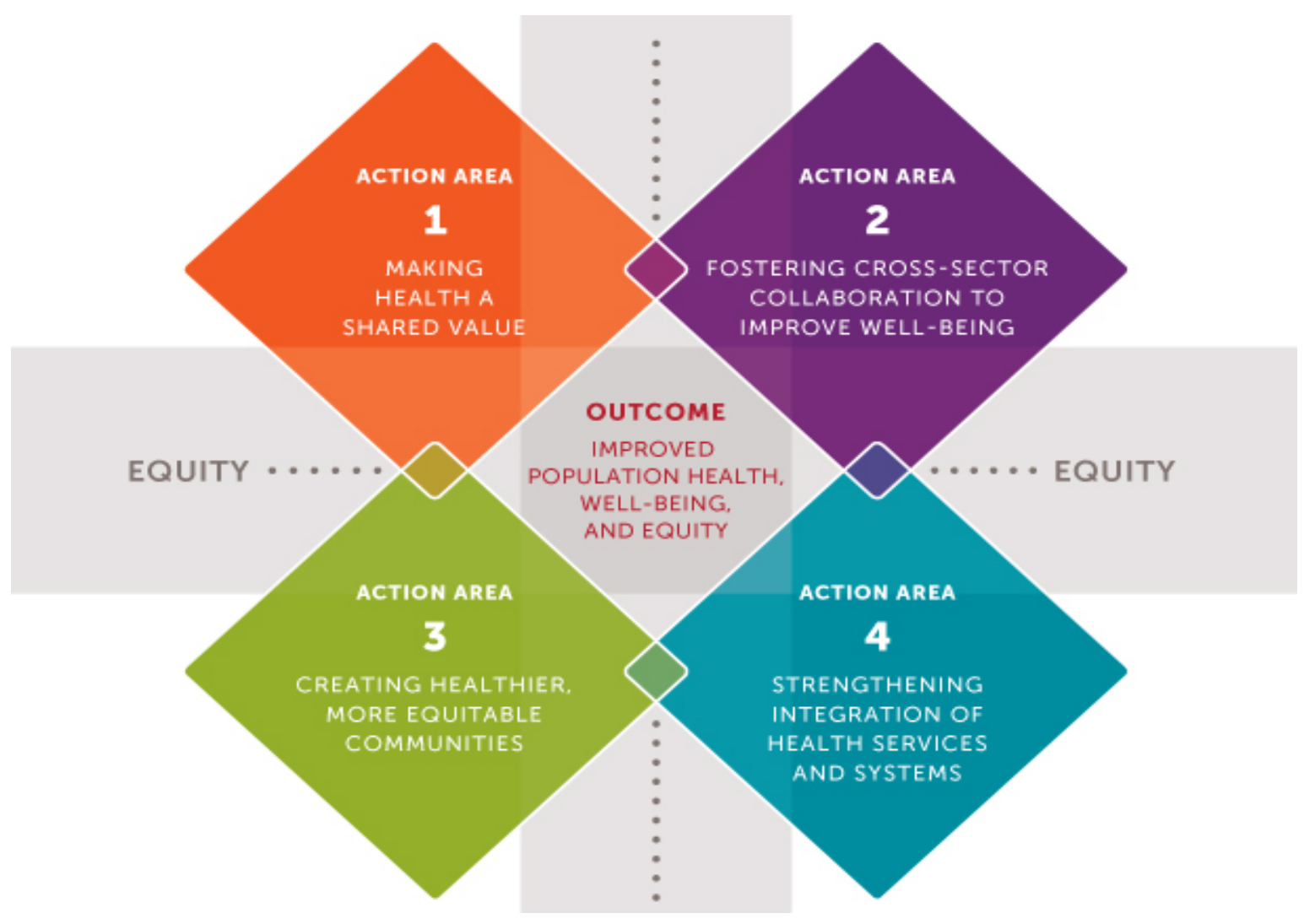

Our process for developing the action framework and translating the conceptual frame of the Culture of Health into something operational leveraged a schema used by members of the study team in developing both community resilience and civic well-being operational frameworks (Chandra et al., 2011; City of Santa Monica, 2015). We used a combination of literature review, structured discussion with experts, and other stakeholder engagement to guide development of the operational action framework (Figure S.2). 
Figure S.2. Process for Action Framework Development

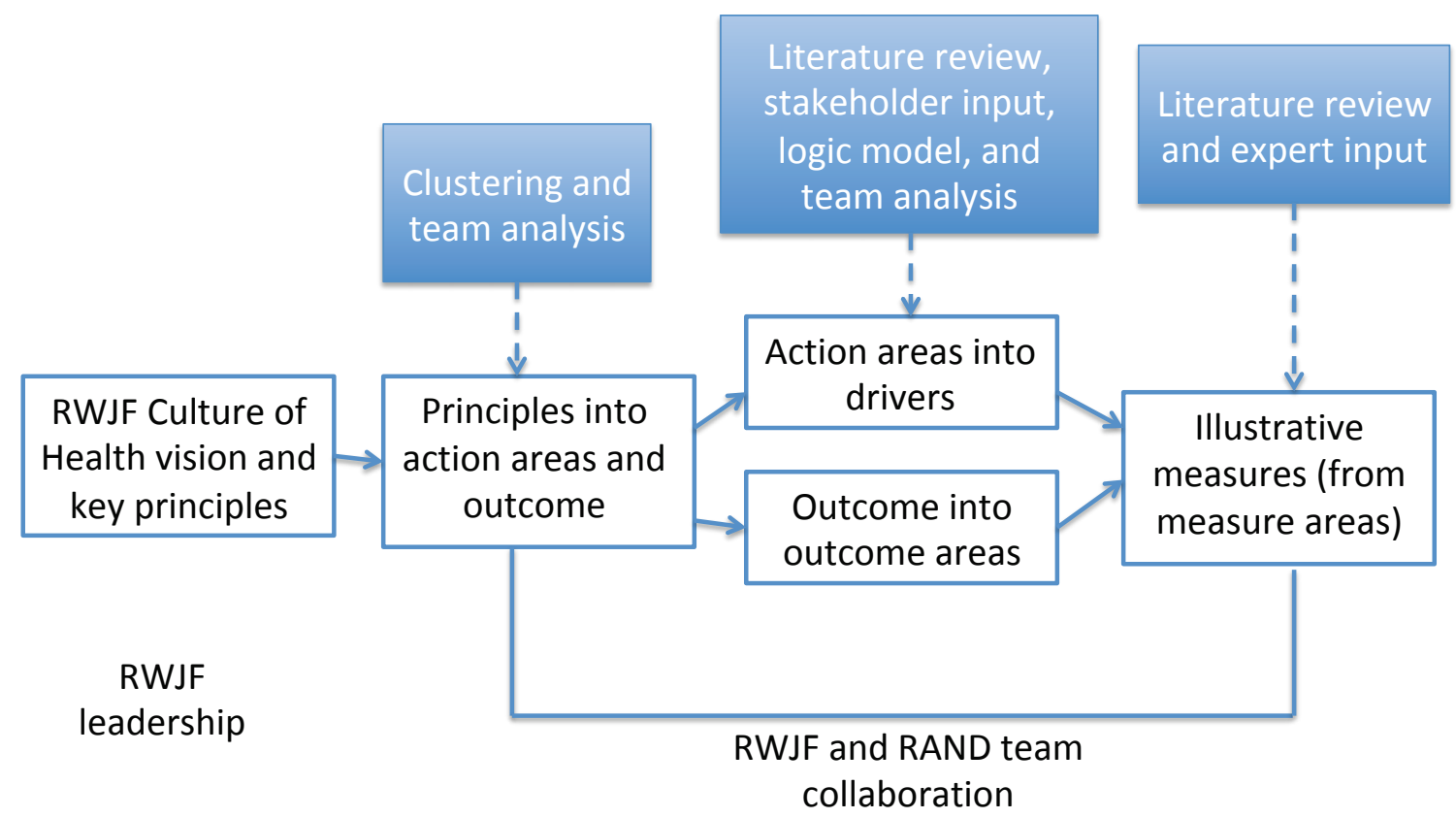

Inputs that informed

development stage

Three analytic steps were used to move from the ten Culture of Health principles to the action framework. First, we examined how principles may cluster together. Team members independently organized the principles into clusters, then iterated as a team to review and name these clusters or action areas. After the principles were grouped, the team created notional logic models to determine how action areas would link together to drive or influence a Culture of Health. The exercise ensured that our choice of groupings aligned with available evidence (e.g., outputs and outcomes related to the evidence of what success may look like in an action area) and were conceptually clear. Finally, as part of a concept mapping effort with stakeholders, we checked our assumptions about the action areas - specifically, to determine whether the conceptual groupings we had recommended were useful and understood by a diverse set of stakeholders. 


\section{Development of Drivers and Illustrative Measures}

To develop drivers and measures for each area in the action framework, the team conducted an environmental scan (literature review), completed a stakeholder engagement process that included input from RWJF partners, developed logic models and other graphics (e.g., creation of figures that illustrated how drivers and measures may relate, narratives of how drivers may work together using community case study examples), and reviewed all progress via team analysis. These four inputs were conducted over a period of approximately one year and employed an intensive, iterative process whereby the RAND and RWJF collaborative team engaged at least once per week by phone and met in person every two or three months.

\section{Environmental Scan/Literature Review}

The team, primarily led by RAND, conducted an environmental scan, which included a review of websites, the peer-reviewed and gray literature (e.g., reports, proceedings), and other materials that summarize issues related to culture, health, and the action areas in the Culture of Health action framework. The scan primarily focused on these topic areas:

1. What is culture?

2. What is health?

3. What are influences for shared values and health, cross-sectoral collaboration, health environment, and health care system effectiveness and efficiency?

4. What are key factors related to complementary areas for a Culture of Health, such as well-being, resilience, etc.?

The scan included a bibliographic search of published and gray literature using such computerized databases as Web of Science, Google Scholar, PubMed, Scopus, Embase, the Cumulative Index to Nursing and Allied Health Literature, and the New York Academy of Medicine's Gray Literature Report. The results of this environmental scan provided insight into the key drivers in the action framework by helping to consider what elements are most relevant to developing a Culture of Health within the action areas, which drivers were most critical for each action area, and what measures require the most progress to achieve the outcomes in RWJF's vision (in short, whether improvement in a measure would have significant impacts on the action area and/or outcome of improved population health and well-being). An initial set of drivers was identified based on the following criteria: 
- relevance and alignment with the action area definition

- indication of an aspect of the action area that needs attention, investment, and/or improvement in order to cultivate or develop a Culture of Health

- ability of the driver to be used by or to be relevant to diverse stakeholder groups that need to engage to activate or cultivate a Culture of Health

- potential link between the action in a driver and available ways to measure change (the linkage could also provide an opportunity for dialogue among key sectors needed for health improvement)

- consistency with relevant or aspirational models or frameworks from other countries conducting work in population health and well-being.

We used the linkages among drivers within and across action areas to further parse the outcome of improved population health and well-being into three outcome areas. In short, we examined what ways change in the driver would lead to change in the outcome (e.g., whether it would change cost or well-being).

While the environmental scan was important for informing the drivers and measures development process, it was not the only input that informed the selection of drivers and measures. We describe the other inputs in the following sections.

\section{Stakeholder Engagement}

The stakeholder engagement process was primarily used for review and vetting of the drivers for the action framework. Stakeholders also provided some input on the measures to consider, but the weight of stakeholder analysis was used more for action framework review. There were four primary steps of stakeholder engagement:

1. review by the RWJF Board of Trustees subcommittee developed for this work

2. consideration by the RWJF network, which includes a diverse leadership network and presentations with relevant professional associations

3. discussion with national and global stakeholders

4. additional expert review.

The RWJF board subcommittee included five board members who represented the health, business, government, and academic sectors—Julio Frenk, Patricia Gabow, Jeffrey Koplan, Robert Litterman, and Peter Orszag. These subcommittee members were engaged throughout the 
entire action framework, drivers, and measures development process. Every quarter, over more than a year, the team presented progress on the action framework and solicited input on the action areas, proposed drivers, and, ultimately, illustrative measures. The board members helped the team to review driver candidates (there were usually about six to eight options per action area) to facilitate selection of the final three for each action area (or outcome area for the outcomes). ${ }^{1}$ In addition to the RWJF board review, we shared the action framework and considerations about drivers with members of the RWJF professional leadership network, a diverse online community of RWJF key partners representing research, practice, and policy fields. The third set of inputs came from stakeholder input outside of RWJF, primarily through focus groups and key informant interviews. We used those stakeholder inputs at the start of the process to review the action framework and action areas, and then throughout the first six to eight months of the effort to ensure that our consideration of drivers was aligned with public priorities. While we also solicited ideas for measurement from them, the stakeholders were primarily used for action framework and driver input.

Finally, we used additional expert review in two ways. First, we had a team of five wellestablished peer reviewers for the work: Nancy Adler, Sandro Galea, David Kindig, Michael Rodriguez, and Stephen Shortell. These experts, who reviewed the initial action framework and provided extensive comment on drivers and measures, were selected because they represented public health and clinical fields, as well as academic and practice experience. Additionally, we engaged several of RWJF's partners to inform the development of the action framework and drivers.

In addition to the literature review and stakeholder engagement, the team utilized the inputs from other research summaries commissioned by RWJF for this effort. These inputs were used in driver and measures selection.

\section{Logic Model Development}

The action framework development process also was guided by understanding the ways in which the drivers and then the final set of Culture of Health measures would catalyze, guide, and assess progress toward achieving a national Culture of Health. Developing a logic model helped

\footnotetext{
${ }^{1}$ We targeted three based on practical feasibility and RWJF communication expertise suggesting that people cannot remember too many items - specifically, no more than three.
} 
the team explore how the drivers and potential illustrative measures may relate to each other (i.e., inputs, outputs, outcomes).

\section{Team Analysis}

The RWJF and RAND team used all of the inputs from the literature review/environmental scan, stakeholder inputs, expert review, and logic model creation to develop and fully inform the action framework, action areas, selected outcome, and associated drivers. The team used collaborative tools (e.g., online sharing sites, web presentations that allow comments in real time) to discuss action areas and drivers, initially dividing RWJF-RAND teams by action areas, and then coming together every week (and in person every few months) to present to each other. These team analysis sessions were critical for ensuring that we had thoroughly discussed the literature and expert inputs and identified priority drivers. In some cases, structured listing and sorting activities and independent team member prioritization processes aided us. Because the team intentionally included diverse representation by expertise (e.g., health services, epidemiology, economics, clinical, public health, social sciences), we were vetting drivers and, ultimately, measures through purposeful, multidisciplinary lenses.

\section{Measures Development}

The action framework provided a launching point for framing and informing RWJF's Culture of Health measurement strategy and measures. The measures were intended to capture key aspects of the action areas and drivers identified in the prior phase. While the core momentum for building a Culture of Health is derived from the action framework, the measures illustrate progress and spark dialogue about the many factors that influence and improve health. The measures are not meant to delineate every indicator of population health but, rather, to represent key elements of possible change.

Before we approached specific measures selection, we defined a measurement strategy and the principles for deciding which measures to include. One of our overarching goals in articulating a measurement strategy was to ensure that the ultimate measurement set represented each of the five parts of the action framework and the specific complementary drivers of interest within each action area. Several goals guided the overarching measurement strategy: 
- Measures needed to represent constructs that possessed demonstrated (or highly plausible) links to important health and well-being outcomes and also had some evidence about the types of interventions and resources needed to drive change in these measures. For example, we considered both the evidence linking complete street policies (i.e., every transportation project should make the street network better and safer for drivers, transit users, pedestrians, and cyclists) to health and well-being outcomes and the evidence of feasible strategies/interventions for implementing complete street policies. These steps were critical to finding actionable measures with the potential to change health and wellbeing within the next five, ten, or 20 years.

- Second, we set the goal of selecting six to ten measures for each action area, roughly distributed across each of the three drivers in each action area to generate a total of 30 to 40 specific measures. This number was selected based on other national measurement efforts suggesting that more than 40 measures would be prohibitive and difficult to track and remember, and six to ten measures would reflect balance among the action areas.

- Third, each measure had to apply at a national level or reflect national-level norms, interventions, or results. For instance, county-level measures of walkability in all 50 states could be aggregated to achieve a nationally representative measure of walkability and could be applied at county or organizational levels as well.

- Finally, the measurement strategy needed to address issues of equity, both in how the strategy would subsequently be implemented as well as in the selection of measures to represent progress to address long-standing disparities. In the context of this work, equity meant two things. First, measures needed to allow for examination by race/ethnicity, income, and so forth. Second, the team included some measures that addressed social policy issues that had historically impeded health progress (e.g., residential segregation).

\section{Measure Characteristics}

What measure characteristics best reflect this strategy? First, measures had to be appropriate for use in diverse geographies, cultures, and ages (context and populations). Second, taken together, the grouping of measures had to reflect a mix of drivers of change and the actual indicators of change (i.e., outcomes). Third, the grouping of measures had to represent outcomes that were immediately achievable and those that were more aspirational to be achieved over a 
generation. Finally, the measures had to be placed in data monitoring and use contexts so that measures could be convincingly linked to data sources now or in the future and could be monitored over time.

\section{Selection and Development of Measures}

Based on the measurement strategy, the team undertook a process to identify associated measures. Our criteria for evaluating individual measures (i.e., "individual-level criteria") included such factors as action area relevance and validity, and criteria to evaluate the full set of measures (i.e., "portfolio-level criteria") included factors such as balance across drivers and accessibility to diverse stakeholders and sectors.

The team created spreadsheets for each action area, sorting prospective measures by drivers and listing information on each measure's source and prior use. We moved through several steps of review, first within action area teams, then as a full project team. We also assigned action area team members to review the measures from action areas other than their own in order to assess the lists and potential selections. We convened as a full project team weekly to present measures and candidate selections. We also conducted a series of exercises to review the measures by action areas and then as a full portfolio. This included various scenario reviews of how measures connected together, narrative creation (i.e., identifying the story of the measures together and how it maps to the action framework), and whiteboarding activities (e.g., putting the measures on cards and then documenting logic flows of how measures in action areas would lead to change in outcome areas).

The remaining sections of this summary describe each action area or outcome area, with attention to definition of the action area, brief discussion of drivers, and a list of the ultimate measure areas. More detail on selected measures within those measure areas are offered in the respective chapters.

\section{Action Area: Making Health a Shared Value}

This action area focuses on the degree to which health is a shared value among individuals and the extent to which individuals feel a sense of interdependence. In this context, shared values mean that individuals and whole communities prioritize health and that health informs and drives local decisionmaking; that communities have high expectations for their environment, health 
system, and supporting services; and that people understand that their health influences and affects others, and vice versa. Accordingly, people are called to action to make decisions that promote not only their own health but also the health of those in their communities. Shared values among individuals can influence shared values among organizations, so making health a shared value is a multilevel process. It includes relationships among neighbors and family members, among organizations, and between individuals and organizations. The research guiding this action area relies heavily upon concepts related to social networks, community resilience, well-being, behavioral economics, and asset-based community development.

Three drivers are critical for ensuring that health is a shared value: mindset and expectations, sense of community, and civic engagement. The sentiment that a shared value of health (i.e., prioritizing health, thinking that it is something to be valued) is a prerequisite for building a Culture of Health was a theme common across the environmental scan, stakeholder input, and RWJF partners. To create a shared value of health, stakeholders and RWJF partners described the need to be able to communicate clearly and improve general population understanding that health is a priority. Creating this awareness would then create the demand for healthy communities, leading to changes that begin to shift the momentum toward a Culture of Health. To fully capture the drivers in this area, we needed to ensure that we had drivers that targeted the understanding of the general population (i.e., mindset and expectations), as well as the ability of a community to mobilize or organize around a demand signal to create health communities (i.e., civic engagement) and/or be influenced by a demand signal (i.e., membership and shared emotion connection created by a sense of community).

The mindset and expectations driver covers the perspectives and views about health held by individuals, families, and organizations. Understanding where community members start in their mindset and expectations will inform where community engagement and information processes may need to start in order to catalyze community health action (Cornish et al., 2014). A strong sense of community is critical for advancing a Culture of Health for two reasons. First, communities that can organize and connect effectively are better able to identify needs and promote action (Morrow, 2001). Second, communities that are strongly connected and have trust in structures, people, and processes can create a foundation that supports a culture (or cultures) of health and recognizes cultural values, norms, and traditions (Kawachi, Subramanian, and Kim, 2008). Relatedly, civic engagement processes in which community members feel that they can 
influence change, and want to influence change, are important to creating shared values for health (Anheier, 2004; Oakley, 1999). Civic engagement is a critical driver toward creating health demand and cultivating complementary plans and incentives that lead to shared results.

Given these drivers, we identified the following measure areas, within which specific, illustrative measures were identified:

- value on health interdependence

- value on well-being

- public discussion on health promotion and well-being

- sense of community

- social support

- voter turnout

- volunteer engagement.

\section{Action Area: Fostering Cross-Sector Collaboration to Improve Well-Being}

The purpose of cross-sector collaboration to improve health and well-being is to optimize the contributions of multiple sectors by linking more traditionally health-focused sectors (e.g., public health, health care, social services) with sectors whose contributions to health and well-being have come into focus more recently (e.g., business, education, faith, housing, law, transportation, zoning). The quality and extent of these partnerships, as well as the nature and extent of investments in these partnerships, should be considered. Cross-sector collaboration holds unmet potential as an engine for aligning the assets, policies, and practices of multiple sectors to (1) improve the health and well-being of organizations, communities, and the nation; (2) improve health equity across diverse racial/ethnic and socioeconomic groups and populations; (3) help entities across sectors achieve their individual goals more effectively; and (4) find novel (and possibly more effective) strategies for fostering population health and well-being.

The view that cross-sector collaboration must be augmented and strengthened was another critical theme common across the environmental scan, stakeholder input, and RWJF partners. Three drivers are critical for ensuring that cross-sector collaboration is achieved: number and quality of partnerships, resources investments across sectors, and policies that support collaboration. These were laid out in a three-part sequence, whereby sectors would know their 
roles and contributions, work effectively together, and have the resources and policies to support ongoing collaboration.

For number and quality of partnerships, we asserted that in order to strengthen cross-sector collaboration, we should focus on the number and extent of collaborative partnerships between health and non-health sectors (number and extent of collaborative partnerships) (BaezcondeGarbanati et al., 2006; Pant et al., 2008). In short, it is important both to enumerate specific partnerships between the health sector and other sectors and to document the quality of these partnerships. This driver would also focus on delineating what contributions each sector can bring to a Culture of Health. The second driver focuses on the resource investments across sectors. This includes resources committed to drive partnership activities that produce positive health and well-being outcomes (Wholey, Gregg, and Moscovice, 2009). The third driver examines policy supports for productive cross-sector collaborations, ensuring that there is a structure that will maintain and develop partnerships over time (Barrett et al., 2005).

Given these drivers, we identified the following measure areas, within which specific, illustrative measures were identified:

- local health department collaboration

- opportunities to improve health for youth in schools

- business support for workplace health promotion and a Culture of Health

- U.S. corporate giving

- federal allocations for health investments related to nutrition and indoor and outdoor physical activity

- community relations and policing

- youth exposure to advertising for healthy and unhealthy food and beverage products

- climate adaptation and mitigation

- health in all policies (e.g., support for working families).

\section{Action Area: Creating Healthier, More Equitable Communities}

The vision for this action area is to ensure that the physical, social, and economic environments in which people live, work, and play support the healthy choice being the easy choice for all residents, including those in historically disadvantaged populations — particularly 
minority racial/ethnic populations and those in low-income areas, which generally provide limited resources and opportunities for healthy choices. Recent work has reinforced the importance of addressing structural drivers of risk for poor health outcomes (Braveman, 2014). Some work has identified racial residential segregation in the United States as perhaps the most fundamental determinant of differences in access to social and economic resources, from schools to economic retail to services and activities that promote health behaviors (Acevedo-Garcia and Lochner, 2003; Acevedo-Garcia et al., 2003; Williams and Collins, 1995).

Social and physical aspects of neighborhoods, including maintained sidewalks for walking, well-designed and well-run programs at parks, and quality after-school programs, can encourage residents to engage in everyday physical activity and "active transport," such as walking or cycling to work. Research has found associations between greater distance to parks and less engagement in physical activity and sports activities (Boone-Heinonen et al., 2010), as well as mental health outcomes (Sturm and Cohen, 2014). Given the importance of such large-scale investments and resources, assuring the opportunity for healthy choices for all in our diverse nation requires efforts at the community and organizational levels that recognize how the health of individuals is influenced by "upstream" factors, which represent many non-health sectors, including (but not limited to) housing, transportation, employment, and education.

Based on the literature review and stakeholder analysis, we identified three primary drivers for creating healthier, more equitable communities: (1) the built environment and physical living conditions, (2) the social and economic environment, and (3) policy and governance. We finalized these three as primary drivers because the literature review and stakeholder input noted that more investment and progress needed to be made in the physical, social, and economic aspects of community and that decisionmaking structures to effectively catalyze and maintain progress of each aspect were often missing. The first two drivers focus on the extent to which health-promoting physical, social, and economic environments are in place and equitably distributed. The third driver spotlights policies aimed at creating healthy environments, with an emphasis on collaboration between residents and large government and corporate institutions.

Given these drivers, we identified the following measure areas, within which specific, illustrative measures were identified:

- housing affordability

- access to healthy foods 
- youth safety

- residential segregation

- early childhood education

- public libraries

- complete street policies

- air quality.

\section{Action Area: Strengthening Integration of Health Services and Systems}

The vision for this action area is a coordinated, overall health system that would balance and integrate health care, public health, and social services and systems. This system would be patient- and family-centered, assessment-driven, evidence-based, and team-based to meet the needs of all ages to improve health and well-being while minimizing the caregiving burden and enhancing the caregiving capacity of families and other support persons. It would address interrelated medical, social, developmental, behavioral, educational, and financial needs to achieve optimal health and wellness outcomes provided in the context of a health team (real or virtual) that has established working relationships with families, clinicians, community partners, and other professionals (Antonelli, McAllister, and Popp, 2009). The importance of social services (and the lack of integration with the health care system to date) may help to explain why the United States spends more on health care than other Organisation for Economic Co-operation and Development (OECD) countries, spends relatively less on social services, and has worse health outcomes (Bradley and Taylor, 2013).

We identified three drivers for this action area: access, consumer experience and quality, and balance and integration. We finalized these three as primary drivers because the literature review and stakeholder input noted that these three areas were key to improving early use of health care services that are preventive, driving more balance in health care costs (a key outcome area), and maintaining the country's focus on high-quality health care. Further, the balance and integration driver was specifically included to prioritize interest in balance between health care, public health, and social services, a feature also being examined vigorously worldwide. Access refers to the ease of engaging in health and related ancillary social services. It was selected as a driver because it has been consistently linked to decreased morbidity/chronic disease burden, mortality, and health inequities; better quality of life; and overall cost savings (Institute of Medicine, 2001, 
2002). Consumer experience and quality relates to the ease with which consumers can move within and across systems; have clear communication about health, costs, processes, and procedures; and receive care that is high-quality, respectful, and responsive to individual preferences (American Hospital Association, 2013; Institute of Medicine Committee on the Future Health Care Workforce for Older Americans, 2008). Balance and integration refers to integration across public health, mental/behavioral health, social service, and health care systems (e.g., data and information sharing, cost/financing models, shared risk) (Martin and Luoto, 2015; Institute of Medicine, 2014b). This driver highlights the extent to which the country values or prioritizes health, as opposed to an emphasis on recovering from illness, and the extent to which these traditionally separate health-related sectors are operating as a unified health system.

Given these drivers, we identified the following measure areas, within which specific, illustrative measures were identified:

- access to public health

- access to stable health insurance

- access to mental health services

- dental visit in past year

- consumer experience

- population covered by an accountable care organization

- electronic medical record linkages

- hospital partnerships

- practice laws for nurse practitioners

- social spending relative to health expenditure.

\section{Outcome: Improved Population Health, Well-Being, and Equity}

Based on the environmental scan of the nation's progress in terms of achieving RWJF's ten principles of a Culture of Health, we identified three outcome areas (enhanced individual and community well-being, managed chronic disease and reduced toxic stress, and reduced health care costs) within the Culture of Health outcome of improved population health, well-being, and equity. We used these outcome areas to track observed impact if progress is made in the four 
action areas and the related drivers. Given that the drivers are in the action areas, we use the term "outcome areas" to distinguish from "drivers."

The action framework for establishing a Culture of Health emphasizes well-being; thus enhanced individual and community well-being is one of the three outcome areas. The establishment of a Culture of Health is, by definition, a transformation in the way society thinks about health, a result that can only happen when complex, system-level change occurs. Therefore, looking at only traditional health outcomes, particularly ones that track specific disease conditions, is not comprehensive enough in scope (Kahneman et al., 2004; Ward, Schiller, and Goodman, 2014). Second, well-being acknowledges imperfect health-that people with chronic conditions or other illnesses can thrive and be healthy. Managed chronic disease and reduced toxic stress (or traumas that affect brain architecture or chemistry) is the second outcome area. Chronic disease is the leading cause of mortality in the world, and as the population ages, the complexity of those chronic conditions only magnifies (Yach et al. 2004). The human and economic toll of chronic disease on society is significant, including lost productivity, quality of life, and health care costs (Centers for Disease Control and Prevention, 2010). The action framework focuses on reducing preventable disease but also on improving the care and management of existing disease, knowing that many are burdened. Reduced health care costs is the final outcome area. Rising U.S. health care costs affect nearly everyone because costs are borne by employers, governments, and individuals alike. As such, progress in this outcome area will require improving efficiency in care delivery, changing payment, and fostering an individual-level impetus to improve health behaviors (Mokdad et al., 2004; OECD, 2013).

Given these outcome areas, we identified the following measure areas, which contain specific, illustrative measures:

- well-being rating

- caregiver burden

- adverse child experiences

- disability associated with chronic conditions

- family health care costs

- potentially preventable hospitalization rates

- annual end-of-life care expenditures. 


\section{Implementation and Next Steps}

This report provides foundational content for a vision of a Culture of Health, as well as the four action areas of the Culture of Health action framework. The report offers background to outline the conceptual underpinnings of the four action areas, the outcome area, how each is defined, and the drivers in each action area. The initial efforts to define and frame each of the action areas to advance the ten principles of a Culture of Health illustrated opportunities and gaps in current measurement to achieve that end. Given the groundbreaking nature of RWJF's initiative, the team anticipated that there would be limitations in the availability of measures that track progress in such areas as health promotion, community capacity for health, cross-sectoral collaboration, shared values, and well-being development. The environmental scan and first phase of stakeholder engagement underscore this challenge.

Over the next phase of the project, surveillance of sentinel communities will complement national monitoring of the Culture of Health by offering insight into how development of a Culture of Health evolves locally. The sentinel community phase of this project started in late 2015, with data collection lead by RTI International. This phase will be critical to understanding how communities are developing (or not developing) a Culture of Health, what data they use to track that development, and how certain Culture of Health measure data could be collected and analyzed as the national effort moves forward. Sentinel communities allow the team to complement national Culture of Health measures with more granularity on cultural change processes (or barriers), cross-sectoral engagement, and individual and community efforts to change the context in which health policies are developed and healthier conditions are created. The hope is to surface drivers of change and likely signals that allow assessment of how communities move toward a Culture of Health. The sentinel communities are not demonstration or place-based communities, but rather sites for naturalistically testing local Culture of Health measurement and overall progress (see www.cultureofhealth.org for site list).

The team also will work to evolve the measures and build out the larger measurement strategy for the action framework. The measurement strategy includes guidance on how measures are tested and validated (e.g., processes for measures prototyping), instructions on how measures may apply to national and/or local context (e.g., factors to consider in local use or consumption), and insights for how measures are framed and communicated for wider, national consumption (e.g., core audience, key messages). This measurement strategy will need to 
acknowledge structural variables that support measures implementation and long-term use. This includes factors related to sectoral roles and engagement, governance structure and alignment to local processes, and coordination and integration with shared accountability structures nationally and locally. 


\section{Acknowledgments}

The authors are greatly appreciative for the time and insights of the many contributors to this report, including reviewers Ramya Chari of RAND and Matthew Trowbridge of the University of Virginia. We are grateful for the contributions of our RAND team members, Linnea Warren May, Jennifer Sloan, and Sarah Weilant. We also extend appreciation to the wide variety of stakeholders who provided their inputs and perspectives that guided development of the action framework and selection of measures. 


\section{Introduction}

Health and health care are at a crossroads in the United States (Davis et al., 2014; OECD, 2013). This country spends more on health care than any other nation. But Americans are not getting their money's worth. For example, the United States ranks only 26th in life expectancy among 40 of the most developed countries (Bauer et al., 2014; Galea et al., 2011; Link and Phelan, 1995; Mokdad et al., 2004; OECD, 2013). It has become increasingly clear that solutions to U.S. health problems must encompass more than reforms to health care systems. Health is a function of more than medical care. Health behaviors and the social and physical environment in which individuals live and work have a stronger influence on well-being than clinical services alone (Braveman, 2014; Galea et al., 2011).

This emerging understanding and the changing landscape of our nation's health suggest a need to rethink the relationships among all sectors of activity and their role in

\section{What Is a Culture of Health?}

A Culture of Health exists when expectations about the high value of health are shared across sectors and when individuals and organizations have the capacity to

- promote individual and community wellbeing (as defined by physical, social, spiritual, and mental health)

- create physical and social environments that prioritize health

- $\quad$ support access to opportunities for healthy lifestyles and high-quality health care for everyone.

health. This includes reimagining the health sector as one that balances health, public health, and social service systems to yield the highest value to individuals and communities. Such a holistic approach requires a coordinated and integrated health sector in which the full spectrum of care is considered. The definition of health also must be reframed from a negative view-in which health is defined as not needing to seek health care - to a positive view, integrating all aspects of life to support healthy living.

Now, communities are working to redefine what it means to get and stay healthy by addressing the social and physical spaces and conditions in which people live, learn, work, and play - the social, environmental, and economic determinants of health (Acevedo-Garcia et al., 2004; Brownson, Haire-Joshu, and Luke, 2006; Littman, 2010; Thomson, Petticrew, and Douglas, 2003). Recent analyses of the relative drivers of health outcomes (Robert Wood 
Johnson Foundation [RWJF], 2014) underscore the need for investments in nonclinical primary prevention and not simply health care. For example, the RWJF review found that nearly onethird or more of the contributions to mortality and morbidity are driven by behavior (e.g., tobacco use, overweight/obesity), suggesting that a fundamental shift in how individuals and communities prioritize supports for healthier behavior remains critical.

Some of this change in orientation to a broader view of health has been required as demographics shift (e.g., population aging, increase in immigration) (Pol and Thomas, 2000; U.S. Census Bureau, 2010). Putting greater priority on early childhood investments is needed to affect health over the life course, while the burden of an aging population must be acknowledged (Fox, Levitt, and Nelson, 2010). Coordinated efforts to promote wellness and prevent diseases are proliferating among a diverse set of stakeholders, including organizations that are traditionally not focused on health (Bauer et al., 2014; Galea et al., 2011; Link and Phelan, 1995; Mokdad et al., 2004; OECD, 2013; University of Wisconsin Population Health Institute, 2014). And big data — large, varied data sets that are commercially available in real or almost-real time - make it possible to analyze health patterns in unprecedented ways to pinpoint actionable determinants, trends, and outcomes of health and well-being (Abbasi et al., 2012).

These shifts not only signify societal transformation but also provide a platform to disrupt standard practice with respect to health and health care and offer an opportunity for an equitybased approach to address disparities in health and well-being. These shifts can also help catalyze a national movement that demands and supports a widely shared, multifaceted vision for a Culture of Health.

In this context, RWJF laid out a vision for a Culture of Health in which there is a shared value of health, demand for investment in health from all Americans, and solutions for improving health that result from cross-sectoral engagement. This vision resulted in ten core principles, initially delineated in Building a Culture of Health (Lavizzo-Mourey, 2014):

1. Optimal health and well-being flourish across geographic, demographic, and social sectors.

2. Opportunities to be healthy and stay healthy are valued and accessible to everyone across the entire society.

3. Individuals and families have the means and opportunities to make choices that lead to healthy lifestyles and optimal well-being and functioning. 
4. Business, government, individuals, and organizations work together to foster healthy communities and lifestyles.

5. Everyone has access to affordable, high-quality health care-both preventive and remedial.

6. No one is excluded.

7. Health care is efficient and equitable.

8. The economy is less burdened by excessive and unwarranted health care spending.

9. The health of the population guides public and private decisionmaking.

10. Americans understand that we are all in this together.

In the context of these Culture of Health principles, it was important to step back and define both culture and health. By fully examining these two concepts, we were then better able to articulate a framework for operationalizing a Culture of Health. In the next sections, we describe how culture and health are defined.

\section{Defining Culture}

Over the last century, anthropologists, sociologists, and other social scientists have defined culture in various ways (Benedict, 1934; Geertz, 1973; Kroeber and Kluckhohn, 1952; White, 1949). Drawing from this literature, we define culture as the sharing and alignment of beliefs, attitudes, values, and actions across a set of individuals, organizations, and decision environments (e.g., where policies or laws are made). It follows, then, that a Culture of Health is a set of social ideas and practices that promote healthy individuals, households, neighborhoods, communities, states, and nations. These shared ideas and practices are key factors for whether and how sectors work together to improve health environments. We should note that there is not a single, common culture. Rather, cultures can develop in communities, as defined by geographic boundaries and demographic characteristics.

In communities with a strong Culture of Health, we expect to observe individuals valuing good health and making healthy choices about their physical, mental, spiritual, and social wellbeing. In such communities, we also would expect to see such organizations as households, schools, workplaces, businesses, government institutions, and nongovernmental institutions valuing and prioritizing health and the health of the individuals that they might influence. 
Further, we anticipate more opportunities for people to make healthy choices, as well as physical and social decision environments that favor healthy choices over unhealthy ones (e.g., using product choice, pricing, promotion, and placement that favor healthy over unhealthy food choices in grocery stores and restaurant chains). But while a Culture of Health may be commonly defined by the priority that the culture places on health and the existence of coordinated actions to support health, it does not mean that the exact strategies for achieving that Culture of Health in a given community are the same.

A brief review of successful, large-scale social change (e.g., drastic reductions in highway deaths and smoking, increases in recycling) suggests that these changes were not the result of any single intervention or even a small set of interventions. Instead, these shifts arose from different kinds of actors at all levels of society pulling the levers that were available to them because each actor had a vested interest in contributing to the desired outcomes.

\section{Defining Health}

In the preamble to its constitution, the World Health Organization (WHO) defined health as "a complete state of physical, mental and social well-being and not merely the absence of disease or infirmity" (WHO, 1948). On a community or population level, definitions have encompassed the health outcomes of a group of individuals, including the distribution of such outcomes within the group. Kindig and Stoddart (2003) have argued that the field of population health includes health outcomes, patterns of health determinants, and policies and interventions that link the two. Stoto (2013a) noted that "population health is fundamentally about measuring health outcomes and their upstream determinants and using these measures to coordinate the efforts of public health agencies, the health care delivery system, and many other entities in the community to improve health."

Improving population health has generally focused on three components: (1) efforts to address social and environmental conditions that are the primary determinants of health, (2) health care services directed to individuals, and (3) public health activities operating at the population level to address health behaviors and exposures as well as social environments. Epidemiologist Geoffrey Rose (1992) noted that "the primary determinants of disease are mainly economic and social and therefore its remedies must also be economic and social. Medicine and politics cannot and should not be kept apart." The Institute for Health Improvement proposed a 
model that summarizes many of these core elements or determinants of population health (Stoto, 2013b, p. 3). The model describes the interactions among upstream factors (e.g., socioeconomic factors, physical environment), individual elements (e.g., genetics), and health-promoting interventions in not only influencing the likelihood of disease and injury but also the development of well-being. Equity is included and refers to fair access to health care and healthpromoting interventions.

Recently, definitions of what constitutes health and, more critically, population health have focused on the process of health promotion. The Ottawa Charter for Health Promotion defined health promotion as the process of enabling people to increase control over and improve their health (WHO, n.d.). Health is seen as a resource for everyday life, not simply the objective of living. The charter summarized fundamental conditions and resources needed for good health, including peace, shelter, education, food, income, and a stable ecosystem.

\section{The Culture of Health Action Framework}

The Culture of Health action framework, which is the focus of this report, is illustrated in Figure 1.1. 
Figure 1.1. Culture of Health Action Framework

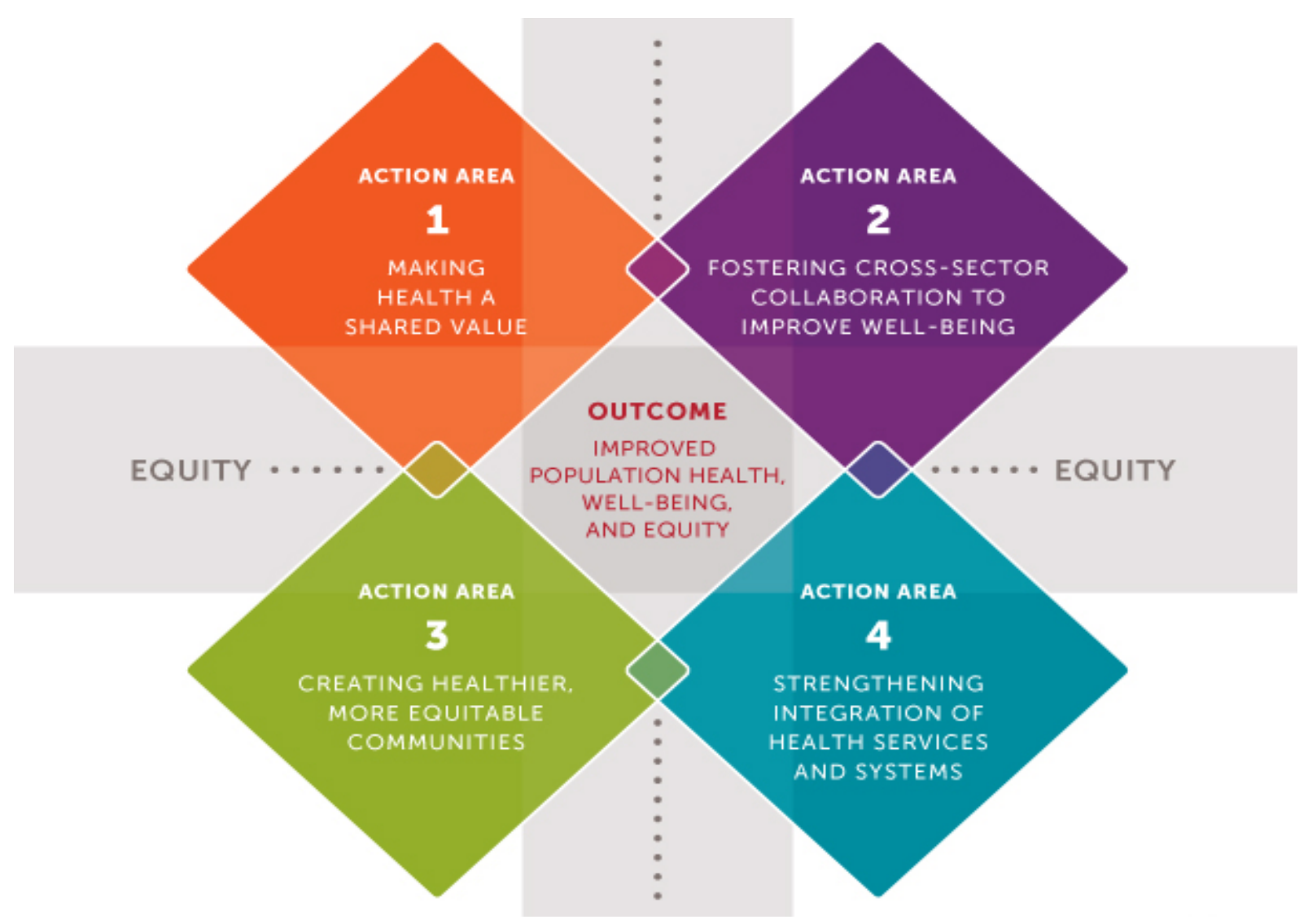

While the remaining chapters will dive more deeply into the development of the action areas and associated drivers and measure areas, we briefly summarize the framework here. For more information about the framework, please see From Vision to Action: A Framework and Measures to Mobilize a Culture of Health (Plough et al., 2015).

The Culture of Health action framework is designed around four action areas and one outcome area. The action areas include (1) making health a shared value; (2) fostering crosssector collaboration to improve well-being; (3) creating healthier, more equitable communities; and (4) strengthening integration of health services and systems. The outcome area-improved population health, well-being, and equity - is intended to serve as the key result of activities in each action area. While not represented in the figure, each action area contains a set of drivers indicating where the United States needs to accelerate change. The drivers provide a set of investment priorities that are deemed critical areas for activity in the action area, which will be needed consistently for sustained improvement in health and well-being. In short, these will not 
change, as the nation will need activity to support the social environment or to improve the mindset and expectations that the public has about health and well-being, for example, over many years. Each action area is also accompanied by a set of measures to illustrate progress in an action area. The measures are meant to offer opportunities for discussion among sectors that need to contribute to health and activities in the action area and to track progress in an action area-and within drivers, specifically. However, they are not intended to holistically capture all of the aspects to measure in a given action area. Chapter Two describes the motivation for each action area.

RAND began its collaboration with RWJF in late 2013 to develop the action framework and ultimately the drivers and illustrative measures. Figure 1.2 illustrates the collaboration and the purpose of the action framework. It should be noted that a Culture of Health is considered a generational or iterative process that will take at least 20 years to develop.

Figure 1.2. RWJF and RAND Collaboration to Develop Action Framework

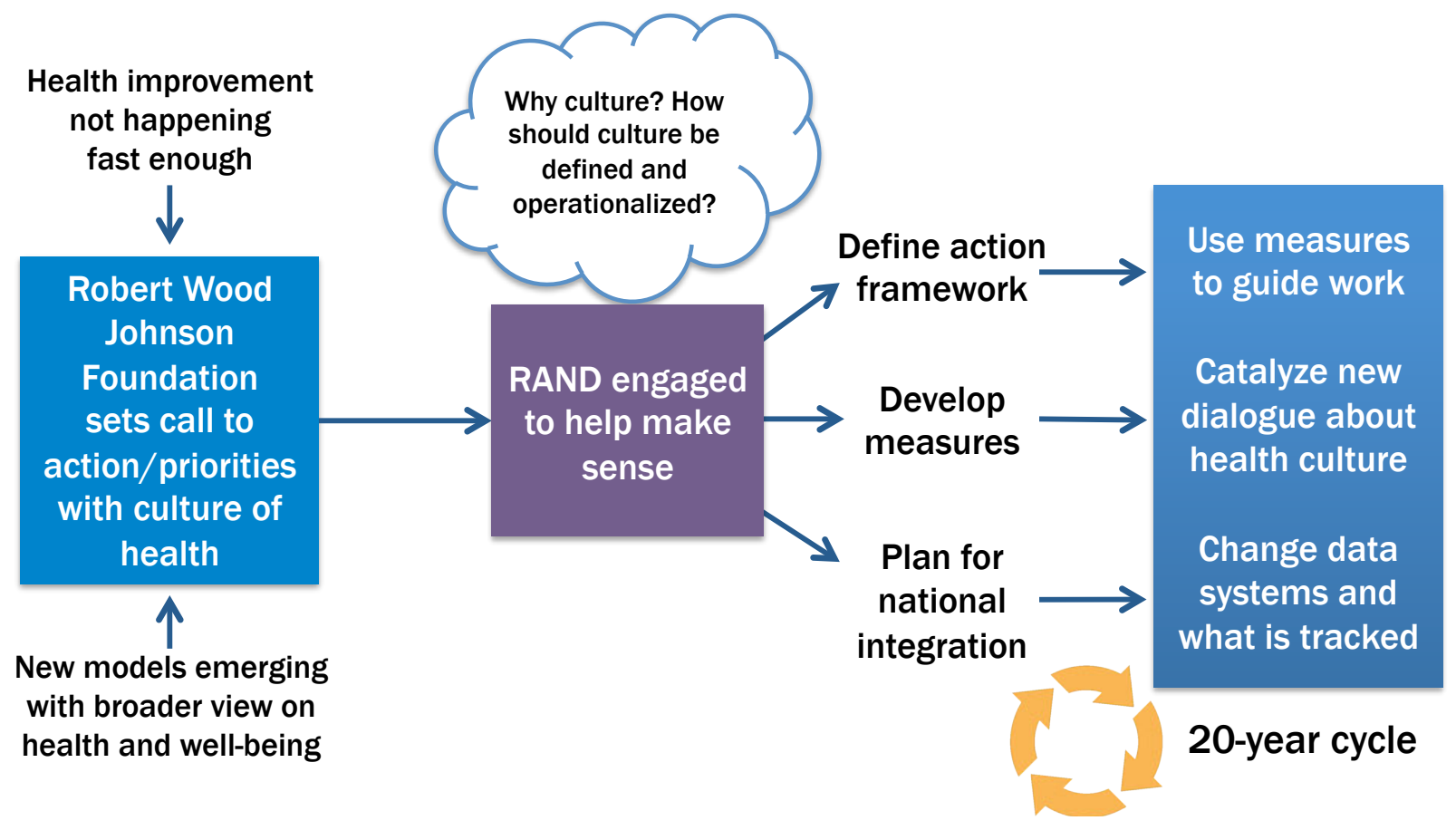

\section{Methods Employed for Action Framework Development}

Our process for developing the action framework and translating the conceptual frame of the Culture of Health into something operational leveraged a schema used by members of the study 
team in developing both community resilience and civic well-being operational frameworks (Chandra et al., 2011; City of Santa Monica, 2015). In short, we used the ten principles of the Culture of Health to guide development of action areas, drivers, measure areas, and illustrative measures. We used a combination of literature review, structured discussion with experts, and other stakeholder engagement to guide development of the operational action framework. This path and these inputs are described in Chapters Two through Four. Figure 1.3 shows how the process unfolded.

Figure 1.3. Process for Action Framework Development

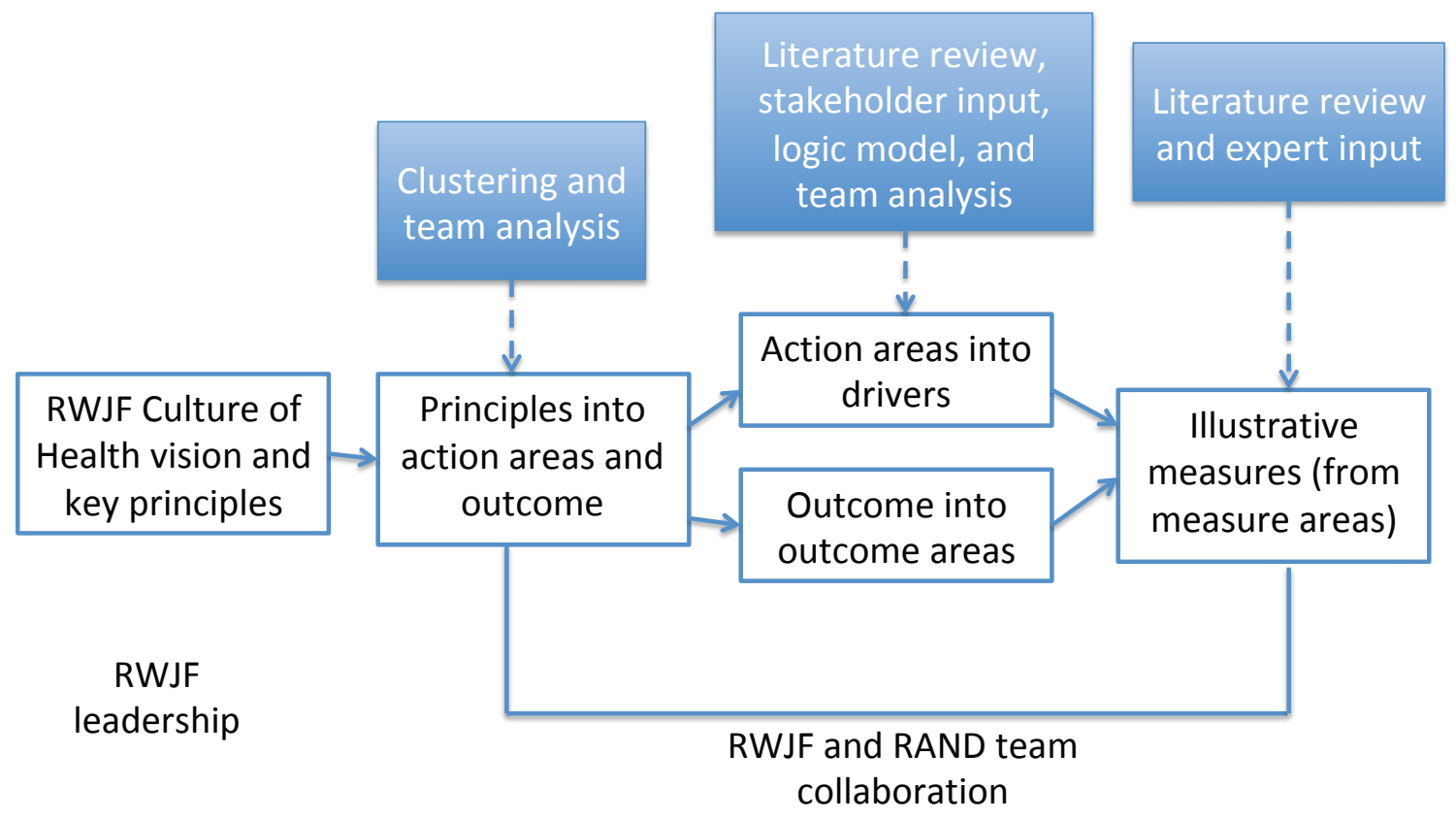

Inputs that informed

development stage

\section{Remainder of the Report}

This report serves as the first phase in developing the Culture of Health action framework, including drivers and illustrative measures. The rest of this report offers additional context for each of the four action areas and the outcomes area, as well as the rationale for the drivers underlying each action area. These drivers are critical because they point to target areas or strategies for advancing a Culture of Health. Each action area chapter also offers the final set of 
measures for each action area. These measures are meant to be illustrative and to signal change, not to serve as a composite Culture of Health index. The report concludes with next steps for the larger measurement strategy development to enable the tracking of Culture of Health development for the next 20 years. 


\section{Developing the Action Framework and Drivers}

This chapter describes the development of the Culture of Health action framework, with attention to the development of the action areas and respective drivers and outcome areas. The next sections detail how the team moved from Culture of Health principles to the action areas, as well as how the team used literature review and stakeholder analysis to inform driver and measures selection. Note that further detail about the stakeholder engagement activities is offered in Chapter Four and also in the longer report on stakeholder efforts (Acosta et al., 2015).

\section{Moving to an Action Framework}

While the definition of a Culture of Health and the ten guiding RWJF principles provided the critical foundation for describing what constitutes the end state of a Culture of Health, RWJF had not yet provided the operational or action framework for how the nation could begin to work toward achieving these outcomes. Since late 2013, RWJF has worked with RAND to develop an action framework for the Culture of Health initiative (shown in Figure 1.2). The ten principles underlying the Culture of Health vision created a starting point for this action framework. The framework consists of clusters of principles organized to show their relationships to each other and to potential actions. The goal of the framework is to convey a holistic, integrated perspective on what it takes to achieve population-level health and well-being. The clusters-which we refer to as "action areas," suggesting broad categories of action-are complex and may act on each other. This framework shows how the action areas influence each other and, ultimately, drive the improvement of population health and well-being. We describe this framework in further detail in the next sections, but first we describe the process used to get to the framework.

\section{Principles to Action Areas in the Action Framework}

Three analytic steps were used to move from the ten Culture of Health principles to the action framework. First, we examined how principles may cluster together. Team members independently organized the principles into clusters, then iterated as a team to review and name these clusters or action areas. Table 2.1 shows how principles mapped to action areas. 
Table 2.1. Map of Culture of Health Principles to Action Framework Action Areas

\begin{tabular}{|c|c|}
\hline Principles & Action Area \\
\hline $\begin{array}{l}\text { Opportunities to be healthy and stay healthy are valued and } \\
\text { accessible to everyone across the entire society }(\# 2) \text {. } \\
\text { Americans understand that we are all in this together (\#10). }\end{array}$ & Making health a shared value \\
\hline $\begin{array}{l}\text { Business, government, individuals, and organizations work together } \\
\text { to foster healthy communities and lifestyles (\#4). } \\
\text { The health of the population guides public and private } \\
\text { decisionmaking (\#9). }\end{array}$ & $\begin{array}{l}\text { Fostering cross-sector collaboration to } \\
\text { improve well-being }\end{array}$ \\
\hline $\begin{array}{l}\text { Individuals and families have the means and opportunities to make } \\
\text { choices that lead to healthy lifestyles and optimal well-being and } \\
\text { functioning (\#3). }\end{array}$ & $\begin{array}{l}\text { Creating healthier, more equitable } \\
\text { communities }\end{array}$ \\
\hline Health care is efficient and equitable (\#7). & $\begin{array}{l}\text { Strengthening integration of health services } \\
\text { and systems }\end{array}$ \\
\hline $\begin{array}{l}\text { Optimal health and well-being flourish across geographic, } \\
\text { demographic, and social sectors }(\# 1) \text {. } \\
\text { No one is excluded (\#6). } \\
\text { Everyone has access to affordable, high-quality health care-both } \\
\text { preventive and remedial (\#5). } \\
\text { The economy is less burdened by excessive and unwarranted health } \\
\text { care spending (\#8). }\end{array}$ & $\begin{array}{l}\text { Improved population health, well-being, and } \\
\text { equity }\end{array}$ \\
\hline
\end{tabular}

After the principles were grouped, the team created notional logic models to determine how action areas would link together to drive or influence a Culture of Health. Detailed models delineated inputs, outputs, short-term outcomes, and long-term outcomes (those logic models are not provided here). Ultimately, we collapsed those models to create the single action framework, illustrated in Figure 1.2. The exercise ensured that our choices of groupings were conceptually clear. Finally, as part of the concept mapping effort with stakeholders (see Chapter Four for details), we checked our assumptions about the action areas, specifically to determine whether the conceptual groupings we had recommended were useful and understood by a diverse set of stakeholders. The stakeholders helped us ensure that our action area names made sense. The stakeholders did not suggest new ways to group the concepts or significant changes to wording of the action areas.

\section{Action Framework Action Areas}

The analytic process described in the previous sections confirmed that the action areas identified in the Culture of Health action framework captured key aspects for advancing a 
Culture of Health in which the outcomes are improved population health, well-being, and equity. The four action areas constitute priority areas of work for the nation and for RWJF, both now and over at least the next generation (i.e., 20 years).

The first action area involves building on shared values of society to forge a common cause around a Culture of Health. It places our nation's values and expectations about health front and center. It emphasizes the importance of achieving, maintaining, and reclaiming health as a shared priority, defined in different ways by different people and communities. Achievements in this action area will fuel a greater sense of community, an increased demand for healthy places and practices, and a stronger belief that individual actions can make a difference in the well-being of others. This action area is seen as foundational to the framework, yet it is the one area that has limited evidence to make informed policy and programmatic decisions.

The second action area concerns working across sectors to improve health. It seeks true collaboration and integration of assets to promote health across traditional health, social, business, economic, and environmental infrastructures. This action area also involves understanding how the systems that support individual and population health operate and how they could be improved and coordinated to operate more effectively. Of course, collaboration and partnerships alone may not be sufficient to change some of the systems that perpetuate poor health; for example, employment may not increase, despite all our partnership efforts. Nevertheless, a community can change a relative portion of social service spending, which ultimately can address or mitigate the challenges of economic conditions.

The third action area involves ensuring equitable opportunities for health and well-being in a wide range of communities and organizational environments. It aims to support residents of all communities to reach their best possible health potential by leveraging the resources of the social and physical environments in which they live, learn, work, and play. By drawing on the value placed on health (action area 1) and on cross-sector partnerships for well-being (action area 2), strategies in this action area will capitalize on people's opportunities for healthy choices, which can reinforce their expectations for what health can be and what well-being is. Ultimately, these changes should produce greater demand for health (as distinct from illness care).

The fourth action area focuses on strengthening integration of health services and systems. This action area encompasses a commitment to equity that ensures individuals' access to highquality, efficient, and integrated systems of public health, health care, and social services that are 
capable of meeting the health needs of a diverse U.S. population across the life span and across the "health span" (i.e., from sick to well). This action area emphasizes general access to and equal opportunity for health care, public health, and social services as essential co-contributors to health and well-being. Further, it addresses the system-level integration and changes that must occur in public health and health care to create an efficient, interdependent system of health and social services.

The fifth area reflects the population health and well-being outcomes of the four action areas. We expect to see improvements in access to care and population health outcomes, economic benefits, and indicators that well-being and productivity are flourishing within all demographic, social, and geographic populations. As a result, we also expect that changes in these outcomes will reinforce the value of health and health care, increasing the value that people place on health for all Americans, and the importance of multi-sector partnerships and changes to achieve the value proposition. In this sense, the action and outcome areas of the Culture of Health action framework are fully interactive.

The previous analysis of what defines culture and health undergirds the Culture of Health action framework as well. For each action area, we considered the levels (individual, organization, policy context) for change to ensure that action was moving toward a culture that is shared and aligned. For example, making health a shared value incorporates the potential for society to build common ideals and expectations around health and its promotion. This action area can encompass population-level or community-level concepts of health, such as sustainability and "green" design on an ecological level, and emotional, social, and physical wellness and productivity on the individual level. Cross-sector collaboration explicitly calls for communication across sectors to integrate and align the promotion of health across organizations. Improved and equitable opportunities for healthy choices and environments underscore the value and importance of choice environments in shaping and sustaining healthy individual and organizational behavior. Strengthened integration of health services and systems will reinforce their roles in improving individual and population health. Thus, activities in each of the four action areas define multiple interacting drivers of a Culture of Health and serve as a theoretical grounding for RWJF's Culture of Health measurement strategy. 


\section{Developing Drivers and Identifying Potential Measures}

To identify drivers and measures for each area in the action framework, the team conducted an environmental scan (literature review), stakeholder engagement inclusive of RWJF partner inputs, development of logic models and other graphic development (e.g., creation of images to show the flow of how drivers and measures may relate, narratives of how drivers may work together using community case study examples), and team analysis. We describe each briefly in the next sections, but our measures development and stakeholder engagement are also detailed further in Chapters Three and Four, respectively. These four inputs were conducted over a period of approximately one year and employed an intensive, iterative process whereby the RAND and RWJF collaborative team engaged at least once per week by phone and met in person every two or three months.

\section{Environmental Scan/Literature Review}

To develop drivers and measures for each area in the action framework, the team, primarily led by RAND, conducted an environmental scan, which included a review of websites, the peerreviewed and gray literature (e.g., reports, proceedings), and other materials that summarize issues related to culture, health, and the action areas in the Culture of Health action framework. The scan primarily focused on these topic areas:

1. What is culture?

2. What is health?

3. What are influences for shared values and health, cross-sectoral collaboration, the health environment, and health care system effectiveness and efficiency?

4. What are key factors related to complementary areas for a Culture of Health, such as well-being, resilience, etc.?

The scan included a bibliographic search of published and gray literature using computerized databases such as Web of Science, Google Scholar, PubMed, Scopus, Embase, the Cumulative Index to Nursing and Allied Health Literature, and the New York Academy of Medicine's Grey Literature Report. Data from relevant articles and websites were abstracted to inform this brief report. Key search terms included cultural change/movement, health, resilience, norm setting, well-being, community health, values, wellness, community action, behavioral change/economics, integrated systems, multi-sector collaboration, and various health drivers. 
The results of this environmental scan provided insight into the key drivers in the action framework by helping the team consider what areas were most relevant to developing a Culture of Health, which drivers were most critical for each action area, and what measures required the most progress to achieve the outcomes in RWJF's vision.

The literature review or environmental scan was summarized in brief descriptions of what should constitute the action area, given the action area definitions described earlier. The full RAND and RWJF team reviewed these summaries, discussed them, and used them as a starting point to identify drivers. An initial set of drivers was identified based on the following criteria:

- relevance and alignment with the action area definition

- ability of the driver to be used by or relevant to diverse stakeholder groups that need to engage to activate or cultivate a Culture of Health

- potential link between the action in a driver and available ways to measure change; the linkage could also provide an opportunity for dialogue among key sectors needed for health improvement

- consistency with relevant or aspirational models or frameworks from other countries conducting work in population health and well-being.

We used the linkages among drivers within and across action areas to further parse the outcome of improved population health and well-being into three outcome areas. In short, we examined what ways change in the driver would lead to change in the outcome (e.g., whether it would change cost or well-being).

While the environmental scan was important for informing the drivers and measures development process, it was not the only input that informed the selection of drivers and measures. We describe the other inputs in the following sections.

\section{Stakeholder Engagement}

The stakeholder engagement process was primarily used for review and vetting of the drivers for the action framework. Stakeholders also provided some input on the measures to consider, but stakeholder analysis was used more for action framework review. We briefly describe the steps of stakeholder engagement here, with additional detail in Chapter Four and Stakeholder Perspectives on a Culture of Health: Key Findings (Acosta et al., 2015). There were four primary steps of stakeholder engagement: 
1. review by the RWJF Board of Trustees subcommittee developed for this work

2. consideration by the RWJF network, which includes a diverse leadership network and presentations with relevant professional associations

3. discussion with national and global stakeholders

4. additional expert review.

The RWJF board subcommittee included five board members who represented the health, business, government, and academic sectors_-Julio Frenk, Patricia Gabow, Jeffrey Koplan, Robert Litterman, and Peter Orszag. These subcommittee members were engaged throughout the entire action framework, drivers, and measures development process. Every quarter, over more than a year, the team presented progress on the action framework and solicited input on the action areas, proposed drivers, and, ultimately, illustrative measures. The board members helped the team to review driver candidates (there were usually about six to eight options per action area) to facilitate selection of the final three for each action area (or outcome area for the outcomes). We decided to identify three items based on practical feasibility and RWJF communication expertise, citing that people cannot remember too many items - specifically, no more than three.

In addition to the RWJF board review, we shared the action framework and considerations about drivers with members of the RWJF professional leadership network, a diverse online community of RWJF key partners representing research, practice, and policy fields.

The third method for generating input was stakeholder engagement, primarily through focus groups and key informant interviews. This included leaders of community-based organizations, governmental agencies, and the private sector and included those focused on health and those in other sectors (e.g., transportation) whose work influences health outcomes. We used those stakeholder inputs at the start of the process to review the action framework and action areas, and then throughout the first six to eight months of the effort to ensure that our consideration of drivers was aligned with public priorities. While we also solicited ideas for measurement, the stakeholders were primarily used for action framework and driver input.

Finally, we used additional expert review in two ways. First, we assembled a team of five well-established peer reviewers-Nancy Adler, Sandro Galea, David Kindig, Michael Rodriguez, and Stephen Shortell. These experts reviewed the initial action framework and provided extensive comment on drivers and measures. These experts were selected because they 
represented public health and clinical fields, as well as academic and practice experience. Additionally, we invited several of RWJF's partners to inform the development of the action framework and drivers. These partner activities, described in the next sections, offered complementary analyses and reports that were used as inputs into the process.

\section{RWJF Partner Inputs}

In addition to the literature review and stakeholder engagement, the team utilized the inputs from other research summaries commissioned by RWJF for this effort. These inputs were used in driver and measures selection.

RWJF convened a group of cultural sociologists to discuss a Culture of Health. In addition, RWJF requested literature reviews on the role of the business community developed by the Vitality Institute and by the Health Enhancement Research Organization (HERO); an analysis of asset-based community development created by Northwestern University researchers; a review of hospital roles in supporting a Culture of Health, including the benefits of community health needs assessment, developed by the Hospital Research and Education Trust (a partner of the American Hospital Association); and a review of the social determinants of health and issues of equity developed by Prevention Institute. We briefly summarize the outputs of each effort in the next sections, though the full reports are provided by those organizations elsewhere (either via RWJF and/or linked to www.cultureofhealth.org). Each input is integrated into action area analysis summarized in Chapters Five through Nine.

\section{Convening Cultural Sociologists}

In May 2014, RWJF convened a meeting of experts in the concept of culture. Chaired by Mabel Berezin, Ph.D., professor of sociology at Cornell University, and Michèle Lamont, Ph.D., Robert I. Goldman professor of European studies at Harvard University, the meeting brought together nine sociologists of culture - academics who have addressed the issues related to culture in areas not necessarily pertaining to health — to discuss their findings with RWJF staff and others supporting the Culture of Health work. In designing the meeting, Berezin and Lamont raised three question areas for discussion:

- How do we build a sense of mutual commitment? How do we create and foster the kinds of social relations that build social solidarities around health? 
- How do we mobilize a response? How do we encourage creative collective engagement in the desire to have healthy communities?

- What messages are needed to communicate that health is a social value?

Meeting attendees prepared background papers on these questions; the papers will be published separately. Key findings include the following:

- RWJF needs to think of multiple cultures, not a single culture, when considering the Culture of Health effort.

- Engagement of the public in a Culture of Health requires information that is easy to understand, transparent, and conducive to dialogue.

- RWJF needs to ensure that it does not focus solely on the elite segments of society. It is important to build trust across social classes for a Culture of Health.

- It will be important for RWJF to build models for change in a Culture of Health based on strength rather than vulnerability.

\section{The Health Enhancement Research Organization}

Both HERO and the Vitality Institute provided keen insights on the role of business investment in Culture of Health development.

HERO is a long-standing, member-driven organization whose members include businesses, consultants, and vendors for employee health, as well as researchers. While HERO has mostly focused on research and resources to improve workplace wellness and employee health management, it recently developed a new workgroup for employer-community health, which informs the RWJF effort. HERO participant-collaborators include the Centers for Disease Control and Prevention, the Samueli Institute, and the Clinton Foundation. RWJF awarded two overlapping grants to HERO to identify cutting-edge tools and strategies for motivating and assisting employers to participate more effectively in community health improvement initiatives. The tactic is based on growing awareness that businesses often offer health and wellness support internally to their employees but have had more limited involvement in external community collaborations and programs to improve the health of the population. This work includes an extended dialogue session with business leaders and experts, a website, a network of engaged leaders, and a summary report built on a scan that HERO conducted in collaboration with the Institute of Medicine. HERO will also implement a "pulse survey" designed to elicit information 
about businesses' community-oriented activities and constructively engage the employer community in community-based health initiatives.

HERO found, and recommended, that the concept of a Culture of Health in the workplace can be measured through (1) environmental audits (i.e., assessment of workplace conditions and features that promote health), (2) self-reported perceptions and satisfaction regarding health programming, and (3) outcomes associated with a Culture of Health (see Table 2.2 for core cultural elements that are important for creating a Culture of Health). HERO leaders recommended environmental audits and self-reported perception surveys to be implemented in employer settings, with outcomes related to a Culture of Health, such as measures of well-being, to be assessed in community settings. Environmental audits would help leaders assess factors that contribute to a Culture of Health, and leaders then could use this assessment to develop strategies for change. Measuring the perception and satisfaction of individuals affected or influenced by a Culture of Health is an important component to the overall measure of the concept. While the environmental audit can gauge the current state of the Culture of Health in the eyes of organizational leaders or human resources stakeholders, the self-reported perception measures determine the success of organizational change efforts. Typically, Culture of Health efforts have been implemented and measured in a very defined employer or organization setting, but measures may be needed to assess the broad population outcomes that can be impacted by Culture of Health efforts. In recent years, measures of well-being have been developed to assess and monitor the health and overall well-being of entire populations, such as happiness or quality of life. 
Table 2.2. HERO Core Elements of Culture That Are Critical for Establishing a Culture of Health in the Workplace

\begin{tabular}{|c|c|c|}
\hline Cor & & \\
\hline $\begin{array}{ll}\text { - } & \text { Norms } \\
\text { - } & \text { Peer support } \\
\text { - } & \text { Shared values } \\
\text { - } & \text { Pushback } \\
\text { - } & \text { Executive leadership } \\
\text { - } & \text { Sense of community } \\
\text { - } & \text { Organizational leadership } \\
\text { - } & \text { Shared vision and mission } \\
\text { - } & \text { Communications }\end{array}$ & 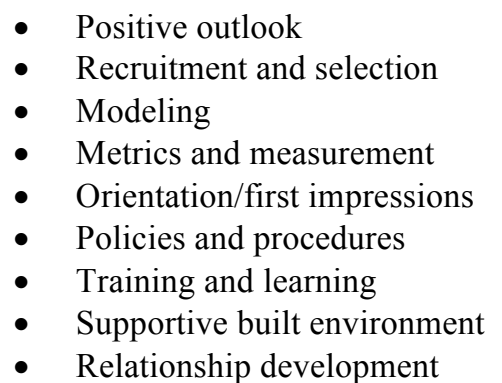 & $\begin{array}{ll}\text { - } & \begin{array}{l}\text { Allocation and commitment } \\
\text { of organizational resources }\end{array} \\
\text { - } & \text { Traditions and symbols } \\
\text { - } & \text { Employee } \\
\text { involvement/empowerment } \\
\text { - } & \text { Internal customer orientation } \\
\text { - } & \text { Rewards and recognition } \\
\text { - } & \text { External community } \\
\text { connections }\end{array}$ \\
\hline
\end{tabular}

\section{The Vitality Institute}

In August 2014, RWJF awarded a nine-month grant to the Vitality Institute with the longterm goal of promoting and incentivizing employer investment in evidence-based community health interventions. The principal aims of this grant were to (1) describe and quantify the business case for employer investment in community health, (2) analyze the current landscape and cost-effectiveness of community evidence-based health interventions led by businesses, and (3) create and disseminate an inventory of best practice interventions that deliver a business return on community investment.

To achieve these aims, the Vitality Institute reviewed the existing research on businesses' return on investment in workplace and community health, interviewed key researchers in the field to expand calculations of the cost-effectiveness of public-sector health promotion interventions, and described and quantified the business case for employer investment in community health. Vitality Institute staff collaborated with consultants from MATRIX Public Health Solutions, Health Partners, Partnership for Prevention, and HERO to review the global literature on the cost-effectiveness of employer-led community health promotion interventions and identify interventions that deliver a business return on community investment. Project deliverables include a report describing the business case for employer investment in community health, an inventory of cost-effective employer-led interventions, and a public event to present these results to business leaders and investors in private and public development and to outline next steps for action (as of the time of this writing [February 2016], the results have not been finalized). 


\section{Asset-Based Community Development}

Researchers from Northwestern University who are leaders in asset-based community development $(\mathrm{ABCD})$ explored different aspects of Culture of Health measurement. $\mathrm{ABCD}$ is a strategy for sustainable community-driven development that emphasizes leveraging grassroots approaches to link micro-assets to the macro environment. RWJF was interested in integrating $\mathrm{ABCD}$ principles into its Culture of Health efforts but first wanted to understand how communities and funders were considering these frameworks. The researchers developed case studies with communities that have employed $\mathrm{ABCD}$ models and with funders that were using $\mathrm{ABCD}$ principles. Key themes from these case studies and interviews were relevant to the action framework, including the development of shared values and social cohesion for health.

Using community case study analyses, the researchers noted that ABCD work must be undertaken in and by the community for real health investments to be realized. Community members need to understand from the beginning that they are not the only ones responsible for implementation and that they will be more effective if they can work with the community, not on behalf of the community. Community members must also have some deeper understanding of the community, including the origins of perspectives about health, which are a key aspect of activating or cultivating a Culture of Health. The research also raised critical questions regarding what entity "owns" health in a community and how shared engagement and accountability can be created.

From funders, researchers found that employing $\mathrm{ABCD}$ values and principles represents a fundamental shift in philosophy, rather than an add-on to an existing programmatic approach. This shift in philosophy also changes how the funder interprets community engagement. Further, the motivation for adopting an $\mathrm{ABCD}$ philosophy has come from funders not seeing the desired level or type of return on investment for health activities in communities despite significant financial resources. As such, funders are using $\mathrm{ABCD}$ frameworks to create a shared accountability model, working in partnership with community leaders.

Community case studies and funder interviews underscored the types of considerations that RWJF needed to employ to support Culture of Health change in communities. Taken together, the $A B C D$ analyses suggested that community development orientations could be useful for considering what will support the advancement of a Culture of Health and how people and institutions can bolster this development. These themes were useful for considering factors that 
influence civic engagement (making health a shared value) and equity in the action framework and for informing elements of the measurement strategy (see Chapter Ten).

\section{Prevention Institute: Disparity Metrics and Health Equity}

Prevention Institute reviewed the literature on health disparities and social determinants of health. Their analysis resulted in several outputs that were important to the development of the action framework, drivers, and measures areas. First, the institute created a model entitled "Community Clusters and Factors Related to Health, Safety, and Health Equity." This model included three clusters - people, place, and equitable opportunity — and 12 structural drivers of health. For people, there were three drivers: (1) social networks and trust, (2) participation and willingness to act for the common good, and (3) norms and culture. For place, there were seven drivers: (1) what is sold and promoted; (2) the look, feel, and safety of the community; (3) parks and open space; (4) ways and means of getting around; (5) housing; (6) air, water, and soil; and (7) arts and cultural expression. Finally, the third element, equitable opportunity, was driven by education and living wages/local wealth.

This rubric informed the action framework overall and considerations about equity in the Culture of Health measures. Prevention Institute also conducted a literature review that informed the action areas of making health a shared value and creating healthier, more equitable communities.

\section{Health Research \& Educational Trust (in partnership with American Hospital Association)}

Another RWJF partner, the Health Research \& Educational Trust (HRET), provided insights into how hospitals are creating a Culture of Health. Hospitals play an essential role in the Culture of Health development process. Many hospitals and care systems are partnering with community stakeholders to identify shared goals and develop innovative approaches to address both health status and the socioeconomic correlates of health. HRET evaluated a broad base of existing literature and internal resources, including survey data, research reports, various community and quality award program applications, and case studies; conducted interviews with leaders of innovative hospitals; and pursued an analysis of almost 200 community health needs assessments (CHNAs) and community health improvement plans (CHIPs). 
HRET leaders found that hospitals assume different roles in advancing a Culture of Health depending on their mission, leadership, how they approach targeted versus broad-based interventions, and the degree of collaboration with community partners. CHNA and CHIP content analysis demonstrated that the top health drivers associated with community needs include access to care, preventive/screening services, and chronic condition management. Issues of socioeconomic security, insurance, or health literacy were key but less frequently cited in plans. Health conditions related to chronic disease and physical health problems were most frequently targeted by hospitals in their implementation strategies, likely because they have existing expertise in those areas. Some hospitals were beginning to address societal factors to have a broader, upstream impact on health. However, many of these issues were not addressed because of a lack of resources, an inability to make a substantial impact, conflict with other organizations already addressing the concern, or an incompatibility with existing hospital strategies.

\section{Logic Model for the Culture of Health}

The action framework development process also was guided by understanding the ways in which the drivers and then the final set of Culture of Health measures would catalyze, guide, and assess progress toward achieving a national Culture of Health (see the logic model in Figure 2.1). Logic model refinement may evolve from sentinel communities (see From Vision to Action: A Framework and Measures to Mobilize a Culture of Health [Plough et al., 2015] and Chapter Ten in this report), baseline data collection, and ongoing efforts to further the use and development of measures as a result of lessons learned in initial implementation. However, this logic model served as the foundation for the action framework, drivers, and illustrative measures. Developing a logic model helped the team explore the mechanisms by which action areas, drivers, measure areas, and measures may relate or function, as well as how the Culture of Health approach may roll out over time. The team also developed logic models for each action area but ultimately deemed it easier to combine these into one logic model to guide the work.

The goal is for the Culture of Health action framework to reframe the discussion on health and health care nationally, enhance partnerships to support health (both with and without RWJF), and stimulate both local and national action for health and well-being. As noted in the first column of the logic model, called inputs, the drivers and illustrative measures were informed by 
RWJF's Culture of Health vision, the action framework, and the literature review. Key stakeholders included a range of RWJF staff, advisors, and partners. The core activities going forward would include implementation of this action framework by key partners and stakeholders. The initial realization of this work would be framed in the form of outputs, including work in sentinel communities (described in Plough et al., 2015), ongoing communication, and use of the action framework nationally and by key stakeholders. Over time, the drivers and illustrative measures would guide and signal progress in each of the action areas (see the short- and medium-term outcomes columns within the logic model). Ultimately, the action framework should guide advancements in population health and well-being and result in a significant shift in the national orientation for a Culture of Health (as shown in the long-term impacts column of the logic model). 
Figure 2.1. Logic Model for Assessing Culture of Health Progress
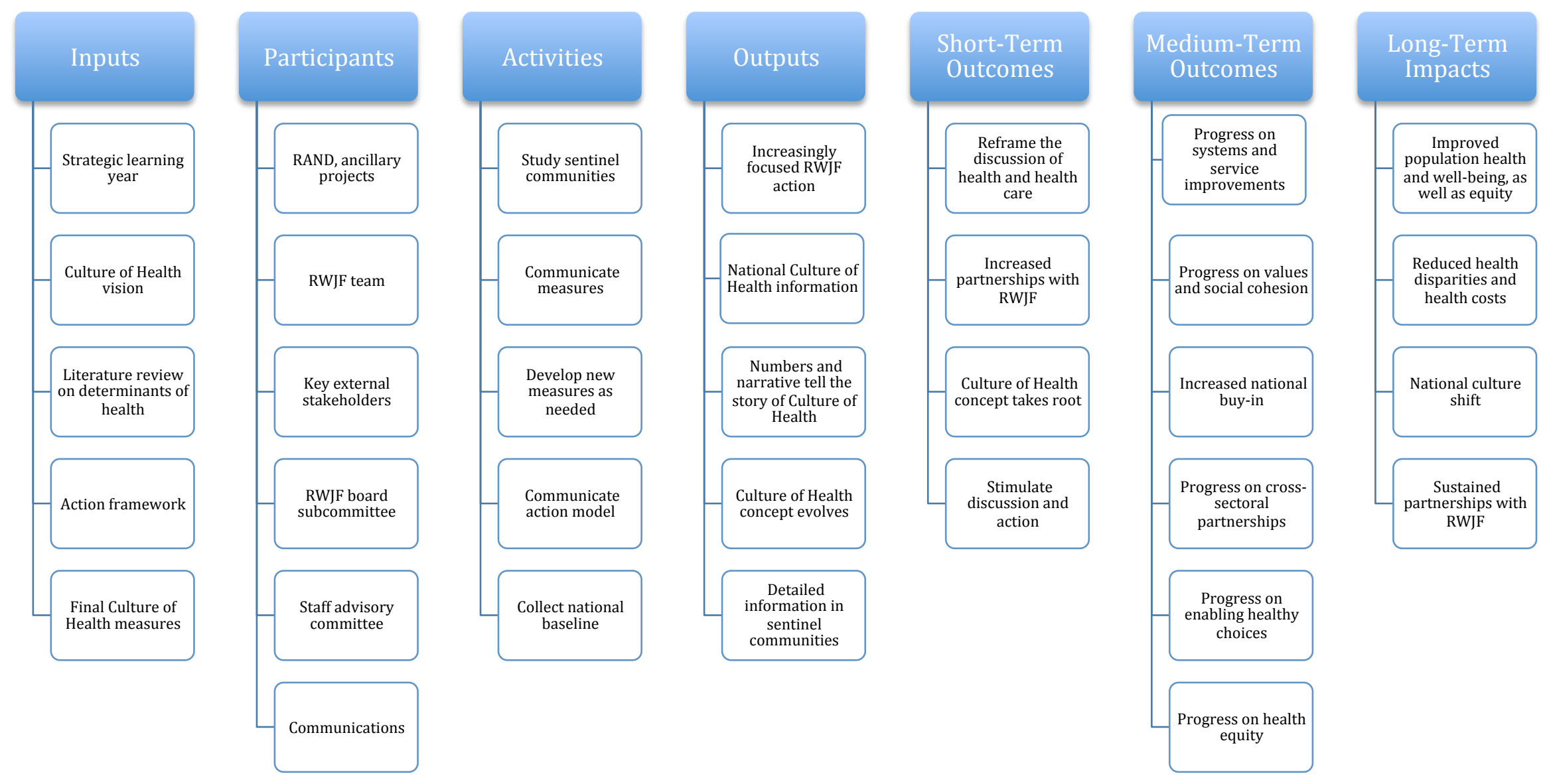


\section{Team Analysis}

The RWJF and RAND team used all of the inputs from the literature review/environmental scan, stakeholder inputs, expert review, and logic model development to inform the action framework, action areas, selected outcome, and associated drivers. Details about the methods employed for measures identification are described in Chapter Three. However, generally, the team used collaborative tools (e.g., online sharing sites, web presentations that allow comments in real time) to discuss action areas and drivers, initially dividing RWJF-RAND teams by action areas and then coming together every week (and in person every few months) to present to each other. These team analysis sessions were critical for ensuring that we had thoroughly discussed the literature and expert inputs and identified priority drivers. In some cases, structured listing and sorting activities and independent team member prioritization processes were employed. Because the team intentionally included diverse representation by expertise (e.g., health services, epidemiology, economics, clinical, public health, social sciences), we were vetting drivers and, ultimately, measures through multidisciplinary lenses.

The next chapters offer more detail on measures identification and stakeholder engagement processes. We then move into action area descriptions by chapter. 


\section{Toward a Strategy for Measuring a Culture of Health}

The action framework (Figure 1.2) provided a launching point for framing and informing RWJF's Culture of Health measurement strategy and measures. In this chapter, we outline the approach to developing this strategy and the selection of the initial set of measures. The measures are intended to capture modifiable drivers of a Culture of Health that link key aspects of each of the four action areas to the health, well-being, and economic outcomes represented in the outcomes area. The measures are not meant to delineate every indicator of population health but, rather, to represent key elements of possible change. In this chapter, we also describe the criteria and process used to guide the selection of the measures that comprise the initial set of Culture of Health metrics (see Plough et al., 2015, and www.cultureofhealth.org for additional measures detail).

\section{Measurement Strategy}

Before we approached specific measures selection, we defined a measurement strategy and the principles for deciding which measures to include. One of our overarching goals in articulating a measurement strategy was to ensure that the ultimate measurement set represented each of the five parts of the action framework, as well as the specific complementary drivers of interest within each action area. Drivers were defined as those components that explained the action area, were selected as points of focus where national emphasis is needed, and offered a grouping structure for identifying measures. We detail each driver in Chapters Five through Nine.

Several goals guided the overarching measurement strategy.

- Measures needed to represent constructs that possess demonstrated (or highly plausible) links to important health and well-being outcomes, as well as some evidence about the types of interventions and resources needed to drive change in these measures. For example, we considered both the evidence linking complete street policies (i.e., every transportation project should make the street network better and safer for drivers, transit users, pedestrians, and cyclists) to health and well-being outcomes, as well as the evidence of feasible strategies/interventions for implementing complete street policies. 
These steps were critical to finding actionable measures with the potential to change health and well-being within the next five, ten, or 20 years.

- Second, we set the goal of selecting six to ten measures for each action area, roughly distributed across each of the three drivers in each action area, to generate a total of 30 to 40 specific measures. This number was selected based on other national measurement efforts suggesting that more than 40 measures would be prohibitive and difficult to track or remember, and six to ten measures would reflect balance among the action areas.

- Third, each measure had to apply at a national level or reflect national-level norms, interventions, or results. For instance, county-level measures of walkability in all 50 states could be aggregated to achieve a nationally representative measure of walkability and could be applied at county or organizational levels as well.

- Finally, the measurement strategy needed to address issues of equity, both in how the strategy was to be subsequently implemented and in the selection of measures to represent progress to address long-standing disparities. In the context of this work, equity meant two things. First, measures needed to allow for examination by race/ethnicity, income, and so forth. Second, the team included some measures that addressed social policy issues that had historically impeded health progress (e.g., residential segregation).

\section{Measure Characteristics}

What measure characteristics best reflect this strategy? First, measures had to represent diverse geographies, cultures, and ages (context and populations). Second, taken together, the grouping of measures had to reflect a mix of change and the actual indicators of change (i.e., outcomes). Third, the grouping of measures should link to outcomes that were immediately achievable, as well as outcomes that were more aspirational to be achieved over a generation. (See Table 3.1 for summary.) 
Table 3.1. Characteristics of Culture of Health Measures

\begin{tabular}{|c|c|}
\hline Measure & Characteristics \\
\hline Context and populations & $\begin{array}{l}\text { - Incorporate aspects of equity and broad sociodemographic and geographic } \\
\text { diversity. } \\
\text { - Cut across varied sectors that influence health. } \\
\text { - Encompass all stages of the life span and health span. }\end{array}$ \\
\hline Drivers and outcomes & $\begin{array}{l}\text { - Address varied known drivers of preventable disease/disability and social } \\
\text { determinants of health, as well as health care quality and public health/health } \\
\text { care/social services coordination. } \\
\text { - Address drivers and outcomes at varying units of analysis or levels of change } \\
\text { (national, state, local, organizational). } \\
\text { - Include a combination of health status outcomes and adoptions of norms, } \\
\text { cultures, and practices. }\end{array}$ \\
\hline Orientation and mix & $\begin{array}{l}\text { - Include those that are used now, developmental, and future/aspirational. } \\
\text { - Be susceptible to change through policy and/or programmatic intervention, } \\
\text { including change in the short and long term. } \\
\text { - Be actionable and meaningful for key actors (users and audiences), including } \\
\text { RWJF staff, grantees, and partners. } \\
\text { - Inspire action and engagement by the general public. }\end{array}$ \\
\hline Data monitoring and use & $\begin{array}{l}\text { - Include accessible and valid data sources. (Note that some measures will be } \\
\text { future-leaning, so validity may be far into the future.) } \\
\text { - Include regular updates (perhaps annually). } \\
\text { - Signal change ("feed-forward") and aspects of confirmed progress } \\
\text { ("feedback"). Feed-forward indicates that a process is in place and working as } \\
\text { intended in terms of trying to address health; feedback indicates whether a } \\
\text { health outcome has been realized. }\end{array}$ \\
\hline
\end{tabular}

It may be helpful to consider measures selection in the context of users and uses of the measures. Users include both national- and community-level leaders across multiple sectors. They include those familiar and utterly unfamiliar with the concept of a Culture of Health, as well as those already invested in the notion. The measures must have strong face validity for a broad range of audiences and must be accompanied by clear, simple, and compelling narrative explanations of their relation to a Culture of Health. In terms of intended uses, the measures are intended to (1) promote understanding of a Culture of Health (enlightenment-oriented uses); (2) inform innovation, strategic thinking, and planning about how to improve the Culture of Health (developmental uses); (3) provide ongoing feedback and support midcourse corrections about efforts to improve the Culture of Health (formative uses); and (4) provide a basis for assessing progress, gaps, and opportunities (summative uses). In short, the measures should clearly support action, not just thinking. 


\section{Criteria for Selecting Individual Measures}

The following section outlines our iterative process for measures review, which included criteria for evaluating individual measures (i.e., individual-level criteria) and the full set of measures (i.e., portfolio-level criteria). Table 3.2 summarizes the priority criteria. The next sections describe the evaluation criteria and steps for applying these criteria in a review process.

Table 3.2. Primary Measures Review Criteria

\begin{tabular}{|c|c|}
\hline Criteria Type & Criteria \\
\hline \multirow[t]{4}{*}{$\begin{array}{l}\text { Criteria for evaluating } \\
\text { individual measures }\end{array}$} & $\begin{array}{l}\text { Action area relevance. Measure can apply to one or more Culture of Health } \\
\text { action areas. Candidate measures not relevant to any action areas were rated } \\
\text { lowest, while those relevant to multiple action areas were rated highest. }\end{array}$ \\
\hline & Validity. Measure is tested or at least has good face and content validity. \\
\hline & $\begin{array}{l}\text { Signal value/catalytic and/or feed-forward value. Measure would provide good } \\
\text { information on national and/or local Culture of Health progress to inform } \\
\text { intervention/improvement processes. }\end{array}$ \\
\hline & $\begin{array}{l}\text { Potential for collaboration. Measure would support or galvanize partnership } \\
\text { between at least two or more sectors (with RWJF where relevant), moving activity } \\
\text { in a sector on behalf of a Culture of Health. }\end{array}$ \\
\hline \multirow[t]{3}{*}{$\begin{array}{l}\text { Criteria for evaluating the } \\
\text { portfolio of measures }\end{array}$} & $\begin{array}{l}\text { Accessible/understandable by a range of audiences. Measures, individually and } \\
\text { as a group, can be explained to and readily understood by users. The measures will } \\
\text { resonate with audiences. }\end{array}$ \\
\hline & $\begin{array}{l}\text { Balance across outcomes, drivers, and (perhaps) important contextual } \\
\text { factors. Measures represent balance across the influencers and the results of } \\
\text { action. }\end{array}$ \\
\hline & $\begin{array}{l}\text { Mixture of risk and protective factors. Measures reflect Culture of Health } \\
\text { vulnerabilities and assets. }\end{array}$ \\
\hline
\end{tabular}

\section{Identifying Culture of Health Measures}

Our process of identifying Culture of Health measures used the action framework and drivers to guide review and ultimate selection (see Chapter Two). As noted earlier, this included extensive literature review, using key words related to the action area and drivers, along with stakeholder analysis (Acosta et al., 2015)

We created spreadsheets for each action area, sorting the initial measures by drivers and listing information on each measure's source and prior use. We then scored these measures 
against the measures criteria summarized above. There was no priority weight given to one criterion over another in the initial inclusion in spreadsheets, though, as noted later, considerations of multiple stakeholder benefits and other criteria ultimately gave certain measures higher ratings in the final analysis (i.e., when the team had to reduce the list).

Those measures that were deemed to have met at least five out of the seven criteria (i.e., to have met the majority of the criteria) were then transferred to the measures repository used by the project team (both RAND and RWJF staff worked collaboratively) for continued review.

Table 3.3 lists the number of measures by action area/outcome area to which serious consideration was given (those in repository):

Table 3.3. Measures by Action and Outcome Area

\begin{tabular}{|l|c|}
\hline Area & $\begin{array}{l}\text { Number of Measures Reviewed } \\
\text { (those transferred to repository) }\end{array}$ \\
\hline Action Area & 68 \\
\hline Making health a shared value & 34 \\
\hline $\begin{array}{l}\text { Fostering cross-sector } \\
\text { collaboration }\end{array}$ & 189 \\
\hline $\begin{array}{l}\text { Creating healthier, more } \\
\text { equitable communities }\end{array}$ & 110 \\
\hline $\begin{array}{l}\text { Strengthening integration of } \\
\text { health services and systems }\end{array}$ & \\
\hline Outcome Area & 74 \\
\hline $\begin{array}{l}\text { Improved population health, } \\
\text { well-being, and equity }\end{array}$ & \\
\hline
\end{tabular}

We moved through several steps of review, first within action area teams, then as a full project team. We also assigned non-action-area team members to review the measures from other action areas in order to assess the lists and potential selections. We convened as a full project team weekly to present measures and candidate selections. We also conducted a series of exercises to review the measures by action areas and then as a full portfolio (described in the next sections in more detail). This included various scenario reviews of how measures connected together, narrative creation (i.e., defining the story of the measures together and mapping it to the action framework), and whiteboarding activities (e.g., putting the measures on cards and then 
documenting logic flows of how measures in action areas would lead to change in outcome areas).

Reviewing Culture of Health Measures

Applying criteria to define and motivate measures review and selection involved several steps, which are outlined in the following flow chart (Figure 3.1).

Figure 3.1. Reviewing Culture of Health Measures

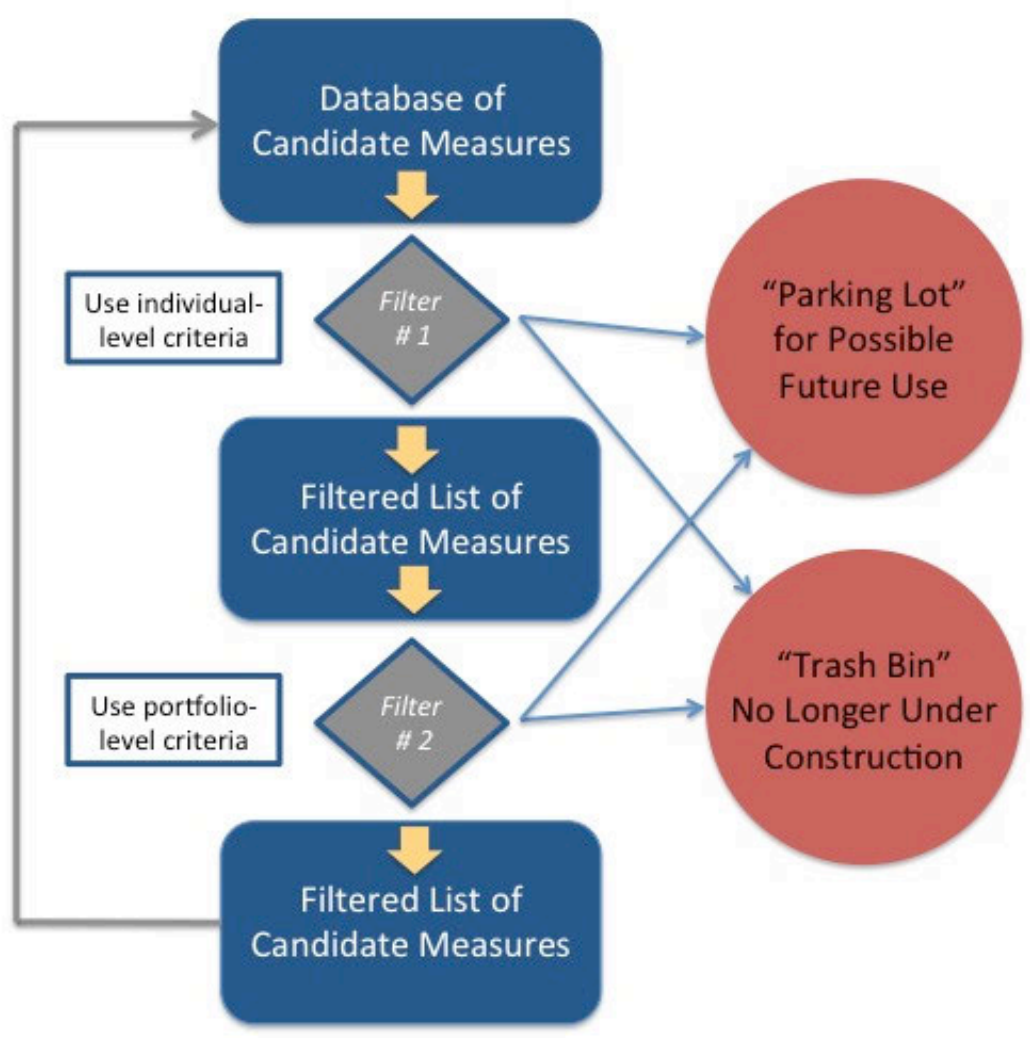

Using the basic steps outlined in the figure, we proceeded through a measures review process. Our ultimate goal was to arrive at a repository of measures that could provide a national picture of a Culture of Health.

Step 1: Initial review of individual measures. The first step of the review involved evaluating each of the candidate measures that were selected for inclusion in a measures repository that was initially developed and maintained by RAND. We identified measures that fit the action areas in 
the action framework as well as the drivers within each action area. Before entering measures information into the measures repository, the team created grids by action area, which delineated the drivers, measure areas, and possible measures within those areas.

After the grid was completed, the list of measures was catalogued in the repository. Each measure was then given a rating of 1 to 5 on each criterion (see next sections for detail) by two raters. If there was disagreement, raters discussed the scoring, though the scores generally aligned well, with only three instances where disagreements were addressed by a third rater. Scores on each criterion, along with an aggregation of scores on all criteria, were then logged in the repository and saved for future use. As summarized in Table 3.2, the criteria included the following:

- Action area relevance. Candidate measures not relevant to any action areas were rated lowest, while those relevant to multiple action areas were rated highest. For example, a civic engagement measure may address multiple action areas, whereas a walkability measure may be too narrow to have wide action area appeal.

- Validity evidence and/or logic-based linkage to Culture of Health action areas. We also determined the extent to which there was evidence linking the measure to Culture of Health action areas. For example, a measure was rated highly if there was plausible evidence (or at least a logic-based argument) that the metric captured (1) the broad construct of a Culture of Health (i.e., the full concept of a Culture of Health), (2) a driver of a Culture of Health (e.g., mindset and expectations), or (3) an important contextual condition that we believed must be present to support development of a Culture of Health (e.g., adequate local/state funds for sidewalk/roadway modifications).

- Developmental progression. The team rated measures on the extent to which they assessed actions that create conditions for future progress (e.g., presence of indoor air quality laws by state). We also sought to select measures that change over time as Culture of Health conditions change or improve and are thus "developmental" with the expectation that measures within each action area of the action framework would evolve over time. For example, we may capture stability of health insurance now, when the Affordable Care Act has been recently established, but if uninsurance or insurance stability becomes less of an issue over time (because we have achieved expansive coverage), we may capture a more nuanced aspect of insurance quality. 
- Signal value or mutability. We also favored measures that link specific actions to specific users (extra consideration was given to measures that had relevance to users in multiple sectors). This criterion was intended to include the notion of "mutability," since this is part of "actionability" for the measurement strategy. In short, a measure area that could change and benefit from intervention was favored higher than a measure area that did not have that value. For example, a measure area such as preventive care use may be more immediately mutable via action steps than, perhaps, altering the state of poverty.

- Potential collaboration. We also rated Culture of Health measures for their distinctiveness from other health measurement efforts by highly rating those measures that could inspire and support partnership for health across sectors. The value of a measure that by its placement in the list can invite conversation and potential action on behalf of two or more sectors was rated more highly (e.g., inviting business and schools to work together).

The team also evaluated measures based on two other practical considerations:

- Current availability of data. We determined that if data were available or could be available, these measures might be most usable for this first set of Culture of Health measures. We also wanted data that allowed for both nationally representative and community-level views, if possible. Measures for which there were both national and community-level data and for multiple years were given the highest rating. Measures for which there were only national data and/or that were available only at a single point in time were given lower ratings. Measures without a plausible data source were given the lowest rating. Measures were also rated on the existence of multiple years of data and the likelihood that the data would continue to be available in the future. Note that while data availability is very important, we did not limit our search and rating to this condition; we considered measures that might help spark or catalyze conversation about the need to collect certain types of information nationally and/or locally.

- Existence of clear and defensible targets/thresholds. Measures were also rated favorably if we were able to define potential good progress (i.e., positive for health outcomes), poor progress, or otherwise (in short, there were established benchmarks). Not all measures were required to have such thresholds, but having them helps users to determine which aspects of a Culture of Health merit attention and action. This would allow RWJF to chart 
change over time, as there may be an ideal state or goal to which a community or the nation can progress over time within the next generation (e.g., with change expected over two to five years, five to ten years, or 20 years).

Step 2: Synthesis of initial review. After we completed step 1, we put measures into one of the following bins, using our cutpoint of meeting five out of seven criteria noted earlier:

- For additional consideration for the first set of measures. Measures that have enough promise to warrant continued consideration for inclusion in the first set of the measurement system.

- "Parking lot" for later consideration. Measures that did not merit further consideration for the first set could be promising candidates for future versions. These may include measures that score high on most criteria but for which existing data sources are weak. A measure's inclusion in the parking lot indicates that it is worth additional efforts and investment in the coming months and years.

- Trash bin. Measures that do not have enough promise to warrant further consideration, either now or in the future.

Note that because the literature review revealed less data on action areas 1 and 2 (e.g., views on health as shared value), the team also considered development of measures where none existed. This resulted in our team developing three new measures (two for action area 1 and one for the outcome area on community well-being within the caregiving burden measure area), which were then fielded in the RWJF National Survey of Health Attitudes (for more information, see www.cultureofhealth.org).

Step 3: Review of portfolio of measures. The team then evaluated the entire portfolio of measures using the following criteria:

- Resonance and meaning for a range of stakeholders. Taken together, the measures needed to tell a story on what mattered for the development of a Culture of Health. Not only is this critical for measurement cohesion, but such a narrative will be important if the general public and community leaders are to build on a shared understanding of what a Culture of Health is and identify and take actions needed to advance that culture. We engaged national advisors for the project in this review and vetting.

- Balance across outcomes, drivers, and (perhaps) important contextual factors. The measures as a whole needed to represent the range of drivers that underlie the action 
areas and the full range of outcome measures that signify improvements in population health and well-being. Given that the measures sought to support developmental, formative, and summative uses, it was useful to have a mixture of measures covering outcomes versus drivers of outcomes. Outcomes were generally more helpful in assessing progress (summative), while drivers can be helpful in guiding innovation (developmental and summative).

- Mixture of risk and protective factors. The measures also included a range of factors that assessed assets, capabilities, and vulnerabilities for development of a Culture of Health. By including a mixture, RWJF and its partners were able to determine targets for investment.

Step 4: Identification of gaps in the current portfolio of measures and targeted searches for new candidate measures that may fill gaps. The final step was a gap analysis, whereby the team repeated the process until it was unable to fill remaining gaps in measurement and the total number of measures was reasonable (i.e., 30-40). The final set consisted of 41 measures.

\section{Summary of Measures Process}

The measures selection process described in the previous sections ultimately included review of nearly 700 measures, of which 475 were included for additional review in the repository. It took 18 months to complete and solidify the final set. The measures review process consisted of several steps, including literature review (described earlier) and stakeholder analysis (described in the next chapter). A more in-depth summary of the stakeholder analysis is also offered in Stakeholder Perspectives on a Culture of Health: Key Findings (Acosta et al., 2015). 


\section{Stakeholder Engagement to Inform the Culture of Health Action Framework}

As described in Chapter Two, several inputs informed the framework and drivers, including stakeholder input from national and global leaders on the action framework, action areas, and drivers. While the full findings from stakeholder engagement are summarized in Stakeholder Perspectives on a Culture of Health: Key Findings (Acosta et al., 2015), we briefly describe key themes here that informed the action areas and the selection of drivers.

Stakeholder engagement served three primary purposes: (1) to support the conceptual development of the Culture of Health framework, including selection of drivers; (2) to support the planning for the naturalistic observations of sentinel communities during the Culture of Health implementation processes (described in Chapter Ten); and (3) to support the identification, development, and use of Culture of Health measures. Stakeholder engagement activities included

- interviews with RWJF partner communities in the United States

- interviews in U.S. communities that were not necessarily RWJF partners, as well as in non-U.S. communities

- "concept mapping" (a technique to delineate the underlying constructs of a given concept) within communities and within health and non-health sectors.

In the next sections, we describe key findings from the interviews and the concept-mapping activities from communities.

\section{Key Principles of Stakeholder Engagement}

Stakeholder engagement efforts were guided by two key methods. First, we utilized both literature and RWJF staff reviews and experience to inform the development of all protocols guiding stakeholder engagement. This included building on the expertise that RWJF's staff and consultants have developed through participation in similar initiatives (e.g., County Health Rankings). Second, the team balanced an evidence-informed perspective and a creative and free elicitation process to promote open dialogue with stakeholders. To operationalize this balance, we used stakeholder engagement activities that began with the definition of Culture of Health 
(see Chapter One), as well as free elicitation activities that were guided by open-ended questions. These guiding principles helped us ensure that stakeholder engagement activities were evidenceinformed, yet flexible enough to encourage open dialogue.

\section{Types of Stakeholders}

We strategically convened stakeholders across the continuum of perspectives, from stakeholders who may be new to concepts related to the Culture of Health to stakeholders who may be leading the country in implementing cutting-edge Culture of Health initiatives. We focused on three types of stakeholders (shown in order of the continuum from most cutting edge to new to the Culture of Health concepts):

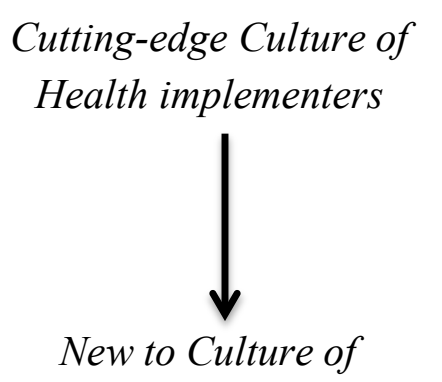

Health concepts
(1) community leaders representing foundations or other wellbeing and resilience groups that were implementing cutting-edge Culture of Health initiatives across a community (2) innovative organization leaders who were implementing cutting-edge Culture of Health initiatives (e.g., linking economic development with health promotion) within their organization (3) organizational representatives from relevant sectors.

Interviews were conducted with stakeholders in 13 communities: Boston, Mass.; Denver, Colo.; Detroit, Mich.; Kansas City, Kan., and Kansas City, Mo.; Los Angeles, Calif.; Louisville, Ky.; Minneapolis, Minn.; New Orleans, La.; Oakland, Calif.; Portland, Oreg.; Sacramento, Calif.; Seattle, Wash.; and Washington, D.C. These communities were chosen because they had organizations engaged in multiple and diverse Culture of Health initiatives, included a mix of health-related community rankings (e.g., high- and low-rated communities according to health outcomes and health factors outlined in RWJF's county health rankings, high- and low-rated communities according to the American Fitness Index), and represented diverse geographic regions.

Initially, snowball sampling was used to identify stakeholders. As a starting point, RWJF provided a table of all the foundation's current and former grantees since 2009. The RAND team narrowed that list down to grantees who were part of national RWJF grant programs, such as Communities Creating Healthy Environments, Leadership for Healthy Communities, Response 
to Economic Hard Times, Active Living by Design, Healthy Kids Healthy Communities, Aligning Forces for Quality, and Roadmaps to Health. In addition, the team considered grantees from other national Culture of Health-type programs, such as the California Endowment's Building Healthy Communities program, and reached out to experts within RAND for suggestions, resulting in a list of 95 communities that were associated with at least one of these initiatives. RAND and RWJF staff reviewed these communities to narrow the list to a smaller set of communities that reflected broad geographic representation, as well as social and demographic diversity. In addition, the research team sought to include communities that were engaged in multiple health-supporting initiatives in order to attract stakeholders with a broad range of experiences. This process was used to identify stakeholders from 12 of the communities. For the 13th community, Washington, D.C., a researcher attended three Mayor's Health in All Policies task force meetings and held individual discussions with key stakeholders participating in the task force. From this list, the research team selected three organizations from each community for initial interviews. The team selected organizations and programs that represented a broad diversity of initiatives, organization types, and populations served and whose work included efforts that addressed building a Culture of Health.

The team also included different organization types and content areas from those covered with the first 13 community stakeholder communities. Organizations in which a team member had a direct contact were prioritized because of a higher level of confidence that the organization's work related to the Culture of Health and the greater likelihood of completing interviews with these organizations within the time frame for data collection. Of note, 53 percent of stakeholders came from the western United States, which may have been a limitation of our snowball sampling approach. We do not believe that unnecessary bias factored into the review based on the geographic distribution, but we did ensure that we included other expert input (e.g., from the RWJF professional network described earlier) from the eastern and southern regions. Further, concept-mapping activities were disproportionately conducted in those regions to balance out the stakeholder interviews.

\section{International Stakeholders}

These stakeholders were purposively sampled from a larger list of organizations generated by the RAND team. This larger list consisted of organizations identified by (1) nominations from 
the team of health-focused and/or community-serving organizations gleaned from their personal experience and professional networks, (2) referrals and nominations by non-RAND researchers based in the geographic regions of interest (e.g., we solicited ideas from researchers who focused on health and well-being in other nations, using our study team networks), and (3) Internet searches for organizations using the keywords "Culture of Health," "health in all policies," "well-being," and "resilience." From this list, the research team then selected a sample for each region (e.g., Global South, Asia), aiming for diversity across these factors:

- organization type (e.g., community-based nonprofits, clinics, foundations)

- geographic focus (international, national, regional, local, neighborhood)

- health and well-being target (e.g., social determinants; a specific disease topic, such as cancer or HIV; or a specific approach, such as increasing access to care or improving quality of care)

- $\quad$ population served (e.g., children, women, or racial and ethnic minorities)

- geographic location

- coverage of the four Culture of Health action areas.

Key Themes from Interviews and Focus Groups

While more detail on stakeholder themes is provided in Acosta et al., 2015, we briefly summarize key themes here. All four Culture of Health action areas were well represented in the work pursued in many of the communities reviewed (see examples in Table 4.1). Some key themes are noted below.

- Making health a shared value: Stakeholders were taking advantage of existing enthusiasm in their communities toward promoting health in diverse ways and embedding "health in all policies." They also used a variety of organizing and community-building strategies to increase a sense of social cohesion and to support Culture of Health initiatives and programs. They noted, however, that community expectations about the value of health varied widely, and community participation in advancing well-being was important, yet often lacking.

- Cross-sector collaboration to improve well-being: In multiple communities, local and state governments were leading efforts to build a Culture of Health. Also, stakeholders were collaborating with partners from diverse sectors, and partnerships among health care centers, local businesses and business organizations, academic institutions, nonprofits, 
and faith-based organizations were leading to creative approaches for dealing with health issues. Stakeholders noted that it was not the number but the quality of partnerships that mattered. They also highlighted that policies that supported maintenance of collaboration were needed.

- Creating healthier, more equitable communities: Most communities were home to at least one program aiming to improve access to healthy eating and physical activity, with many such programs focusing on community and school gardens, as well as walking and biking. Stakeholders noted that the social, economic, and physical environment had to be designed collectively to promote health, but also that communities needed policy and governance structures to sustain health in all policies.

- Strengthening integration of health services and systems: In addition to striving to extend high-quality health care to all individuals within a community, stakeholders were breaking ground in this action area by bringing new topics into the clinical arena, such as assessing patients' social connectedness and educating consumers about health care using innovative metrics and web-based tools. Stakeholders described that the balance and integration of health and human services had to be recognized alongside well-established measures of health care quality.

While most stakeholders felt that evaluating Culture of Health initiatives presented a significant challenge, they discussed them using a variety of process and outcome measures in their initiatives:

- Process measures for community partnerships included such activities as establishing and maintaining memoranda of understanding with partner organizations, with measures focused on the quality and types of community partnerships. When the goal was to change the perception of a health issue, some stakeholders measured community perceptions around the issue and studied how an issue was framed in the media.

- Outcome measures included long-standing health care systems measures, such as the Healthcare Effectiveness Data and Information Set (HEDIS). In the area of community health, some were using newer measures and tools, such as the Health Impact Assessment, Community Healthy Living Index (CHILI) Equity Profiles, and the Healthy Communities Framework, to gain a comprehensive understanding. 
Stakeholders expressed opinions about measurement in general. Some wanted less emphasis on measurement and more emphasis on "boots on the ground" implementation, while other stakeholders, particularly those working in areas outside the traditional boundaries of public health (e.g., the built environment) noted that their fields should emphasize measurement more and build more evaluation. Several stakeholders affirmed that Culture of Health measurement should use an appropriate time frame for change, such as the recommended generational approach that RWJF is pursuing, given that it takes a long time to change the policies that impact health.

Table 4.1. Community Approaches to Building a Culture of Health

\begin{tabular}{|l|l|l|}
\hline Community & $\begin{array}{l}\text { Action Area } \\
\text { Being Used }\end{array}$ & Summary of Relevant Activities \\
\hline $\begin{array}{l}\text { Boston, } \\
\text { Mass. }\end{array}$ & $\begin{array}{l}\text { Strengthening } \\
\text { integration of } \\
\text { health services } \\
\text { and systems }\end{array}$ & $\begin{array}{l}\text { Contact organizations have addressed the equitable distribution of high- } \\
\text { quality health services. Dimock Community Health Center, a federally } \\
\text { funded health clinic, has integrated various approaches to serve mostly } \\
\text { Hispanic and African American patients. Programs included an adult } \\
\text { GED program, an eye clinic to identify diabetics who may need vision } \\
\text { care, and residential behavioral health care. }\end{array}$ \\
\hline Denver, Colo. & $\begin{array}{l}\text { Creating } \\
\text { healthier, more } \\
\text { equitable } \\
\text { communities }\end{array}$ & $\begin{array}{l}\text { Contact organizations worked on increasing opportunities for healthy } \\
\text { lifestyles and access to quality education. The Center for Human } \\
\text { Nutrition and the Stapleton Foundation created the Active Living } \\
\text { Partnership of Greater Stapleton with the Denver Health and Hospital } \\
\text { Authority. This project provides children opportunities to play, walk, } \\
\text { bike, and eat healthy food in lower-income neighborhoods in southwest } \\
\text { Denver. Padres Unidos is a community-based organization that focuses } \\
\text { on education equity, immigrant student rights, and health justice in } \\
\text { schools. }\end{array}$ \\
\hline $\begin{array}{l}\text { Detroit, } \\
\text { Mich. }\end{array}$ & $\begin{array}{l}\text { Strengthening } \\
\text { integration of } \\
\text { health services } \\
\text { and systems }\end{array}$ & $\begin{array}{l}\text { The Greater Detroit Area Health Council educated individuals about the } \\
\text { spending that goes into health care versus social determinants of health. } \\
\text { It also promoted a healthy economy and healthy people by reducing } \\
\text { investments in health care and increasing them in social determinants of } \\
\text { health. }\end{array}$ \\
\hline
\end{tabular}




\begin{tabular}{|c|c|c|}
\hline Community & $\begin{array}{l}\text { Action Area } \\
\text { Being Used }\end{array}$ & Summary of Relevant Activities \\
\hline $\begin{array}{l}\text { Kansas City, } \\
\text { Kan., and } \\
\text { Kansas City, } \\
\text { Mo. }\end{array}$ & $\begin{array}{l}\text { Fostering cross- } \\
\text { sector } \\
\text { collaboration to } \\
\text { improve well- } \\
\text { being }\end{array}$ & $\begin{array}{l}\text { Stakeholders and partners from diverse sectors thought creatively about } \\
\text { how to increase access to preventive care for residents. Collaboration } \\
\text { between health care facilities, universities, the Chamber of Commerce, } \\
\text { nonprofits, and faith-based organizations created a Culture of Health in } \\
\text { Kansas City. For example, the United Way partnered with the fire } \\
\text { department to start an innovative and now widely recognized program in } \\
\text { which first responders connect community members to preventive health } \\
\text { and social service resources (in addition to immediate health needs) when } \\
\text { they respond to } 911 \text { calls. }\end{array}$ \\
\hline $\begin{array}{l}\text { Los Angeles, } \\
\text { Calif. }\end{array}$ & $\begin{array}{l}\text { Creating } \\
\text { healthier, more } \\
\text { equitable } \\
\text { communities }\end{array}$ & $\begin{array}{l}\text { Los Angeles has a number of ongoing health projects that address the } \\
\text { equitable distribution of health care resources and involve collaborations } \\
\text { with multiple sectors. For example, Building Healthy Communities in } \\
\text { South Los Angeles is a ten-year effort of the California Endowment, now } \\
\text { at the midway point. It is devoted to creating a place-based focus in } \\
\text { which organizations work together-some with a long public health } \\
\text { history, others more focused on social justice, advocacy, etc. }\end{array}$ \\
\hline $\begin{array}{l}\text { Louisville, } \\
\text { Ky. }\end{array}$ & $\begin{array}{l}\text { Making health } \\
\text { a shared value }\end{array}$ & $\begin{array}{l}\text { Stakeholders described strong collaboration around and commitment to } \\
\text { building a Culture of Health in Louisville. The city took an innovative } \\
\text { step for a local government in creating a Center for Health Equity, which } \\
\text { works toward reducing impact of social determinants on health through } \\
\text { projects like corner store conversion and after-school programming. The } \\
\text { mayor has also emphasized health in all policies as a priority. This } \\
\text { leadership around Culture of Health-type strategies contributed to what } \\
\text { stakeholders described as a vibrant attitude of collaboration around health } \\
\text { issues in Louisville. }\end{array}$ \\
\hline $\begin{array}{l}\text { Minneapolis, } \\
\text { Minn. }\end{array}$ & $\begin{array}{l}\text { Strengthening } \\
\text { integration of } \\
\text { health services } \\
\text { and systems }\end{array}$ & $\begin{array}{l}\text { Innovative work around health care quality and access has taken place } \\
\text { through City Health Department programs and metrics and evaluation } \\
\text { work at MN Community Measurement. The governor provided } \\
\text { leadership in the form of Healthy Minnesota 2020, a statewide health } \\
\text { improvement framework. }\end{array}$ \\
\hline $\begin{array}{l}\text { New Orleans, } \\
\text { La. }\end{array}$ & $\begin{array}{l}\text { Strengthening } \\
\text { integration of } \\
\text { health services } \\
\text { and systems }\end{array}$ & $\begin{array}{l}\text { New Orleans Insight Center was built on the premise that the pathway to } \\
\text { sustainable growth lies in strengthening the most vulnerable and } \\
\text { economically detached populations. Its work includes research and } \\
\text { analysis, communications, capacity-building, advocacy, and network- } \\
\text { building based on the pillars of inclusiveness, respect, integrity, and } \\
\text { collaboration. }\end{array}$ \\
\hline $\begin{array}{l}\text { Oakland, } \\
\text { Calif. }\end{array}$ & $\begin{array}{l}\text { Making health } \\
\text { a shared value }\end{array}$ & $\begin{array}{l}\text { Organizations with focuses as divergent as environmental toxins and } \\
\text { criminal justice all have an eye toward broader community health and } \\
\text { well-being, and their initiatives are bringing new topics under the } \\
\text { umbrella of health, such as mass incarceration, community resilience } \\
\text { against the effects of climate change, and closing the racial wealth gap. }\end{array}$ \\
\hline
\end{tabular}




\begin{tabular}{|c|c|c|}
\hline Community & $\begin{array}{l}\text { Action Area } \\
\text { Being Used }\end{array}$ & Summary of Relevant Activities \\
\hline $\begin{array}{l}\text { Portland, } \\
\text { Oreg. }\end{array}$ & $\begin{array}{l}\text { Fostering cross- } \\
\text { sector } \\
\text { collaboration to } \\
\text { improve well- } \\
\text { being }\end{array}$ & $\begin{array}{l}\text { A number of projects built connections among organizations from } \\
\text { different sectors, including education, food, health care, and public } \\
\text { health. For example, the Healthiest State initiative in Oregon is pulling } \\
\text { together many leaders to create a Blue Zone-type project for the state. } \\
\text { The idea is to focus on a geographical region or sector (e.g., schools, } \\
\text { food industry) and build momentum for healthy change within it. The } \\
\text { effort aims to embed equity and disparities at the start so that everyone } \\
\text { benefits, not just some people or communities. The partnership was } \\
\text { expected to launch in fall } 2015 \text {. }\end{array}$ \\
\hline $\begin{array}{l}\text { Sacramento, } \\
\text { Calif. }\end{array}$ & $\begin{array}{l}\text { Creating } \\
\text { healthier, more } \\
\text { equitable } \\
\text { communities }\end{array}$ & $\begin{array}{l}\text { The California Endowment-funded Building Healthy Communities } \\
\text { initiative has leveraged partnerships with many local organizations to } \\
\text { address the problem of food deserts (which have very limited access to } \\
\text { full-service grocery stores and an abundance of convenience stores that } \\
\text { do not stock healthy foods); it is promoting gardening in empty lots and } \\
\text { front yards in neighborhoods across the city, along with other strategies. } \\
\text { WALK Sacramento has made substantial progress promoting walking } \\
\text { and biking by collaborating with developers, local districts, officials, and } \\
\text { the community directly. }\end{array}$ \\
\hline $\begin{array}{l}\text { Seattle, } \\
\text { Wash. }\end{array}$ & $\begin{array}{l}\text { Making health } \\
\text { a shared value }\end{array}$ & $\begin{array}{l}\text { A strong community organizing approach to health promotion is taking } \\
\text { place through Alliance for a Just Society, a national network of } 14 \text { racial } \\
\text { and economic justice organizations coordinating to address economic, } \\
\text { racial, and social inequality, and Washington CAN, a } 40,000 \text {-member } \\
\text { grassroots organization that provides advocacy for low- and moderate- } \\
\text { income residents on a variety of health, economic, and social issues. }\end{array}$ \\
\hline $\begin{array}{l}\text { Washington, } \\
\text { D.C. }\end{array}$ & $\begin{array}{l}\text { Fostering cross- } \\
\text { sector } \\
\text { collaboration to } \\
\text { improve well- } \\
\text { being }\end{array}$ & $\begin{array}{l}\text { The Mayor's Health in All Policies Task Force represented a unique } \\
\text { collaboration of district agencies, including the Department of Health, } \\
\text { Department of Environment, Office of the Deputy Mayor for Health and } \\
\text { the Environment, Office of the Deputy Mayor for Education, Department } \\
\text { of Behavioral Health, Department of General Services, Department of } \\
\text { Parks and Recreation, Department of Transportation, Office on Aging, } \\
\text { Office of Planning, and Public Schools. }\end{array}$ \\
\hline
\end{tabular}

\section{Concept Mapping}

To gather stakeholder input on the Culture of Health initiative, the RWJF-RAND research team also worked with Concept Systems to conduct a unique process called concept mapping. The purpose was to gather inputs about what stakeholders consider important to promoting a Culture of Health. Concept mapping brings together multiple ideas and perspectives on a single topic and helps to identify defining themes and priorities for investment or measurement, using 
cluster analysis and multi-action-area scaling (Trochim et al., 2004). For the Culture of Health initiative, concept mapping provided insight into whether the proposed action framework fully encompassed the factors that may influence the development of a Culture of Health (a fuller report is provided by Concept Systems; contact them for more information). It also provided additional support for the priority drivers used in the action framework.

\section{Concept Mapping Phase One}

As a first step, we conducted a small-scale pilot to quickly gather critical inputs from four communities that are considered innovators in concepts related to a Culture of Health: Nashville, Tenn.; Pittsburgh, Penn.; San Antonio, Tex.; and the state of Delaware. The pilot was conducted in two phases. First, stakeholders in each community participated in a brainstorming process using a single, focused prompt: "In a community where people share the view that health is important for everyone, community leaders and decisionmakers of all kinds would ..." (Phase 1). Feedback gathered using this prompt was then consolidated into a single list of unique ideas by RAND and Concept Systems teams. This list of ideas was then sorted and rated by the same stakeholders on importance for promoting a Culture of Health and presence, or whether the idea had materialized in their community (Phase 2). These ratings were then used to cluster ideas into a completed concept map (Figure 4.1). Findings were shared with stakeholders from participating communities via webinar. While concept mapping findings are not presented here in detail, the effort informed (and ultimately affirmed) the selections and groupings of the action areas in the Culture of Health action framework. For example, the concept mapping work showed how shared values align (action area 1), how partnerships link together (action area 2), and how decisionmaking is a part of social determinants (action area 3 and corresponding driver policy and governance). The colors of the clusters do not denote anything specific, but the relative sizes of the clusters signify the frequency with which each theme was cited in group discussions. Clusters with related concepts are located near each other. 
Figure 4.1. Culture of Health Pilot Concept Map

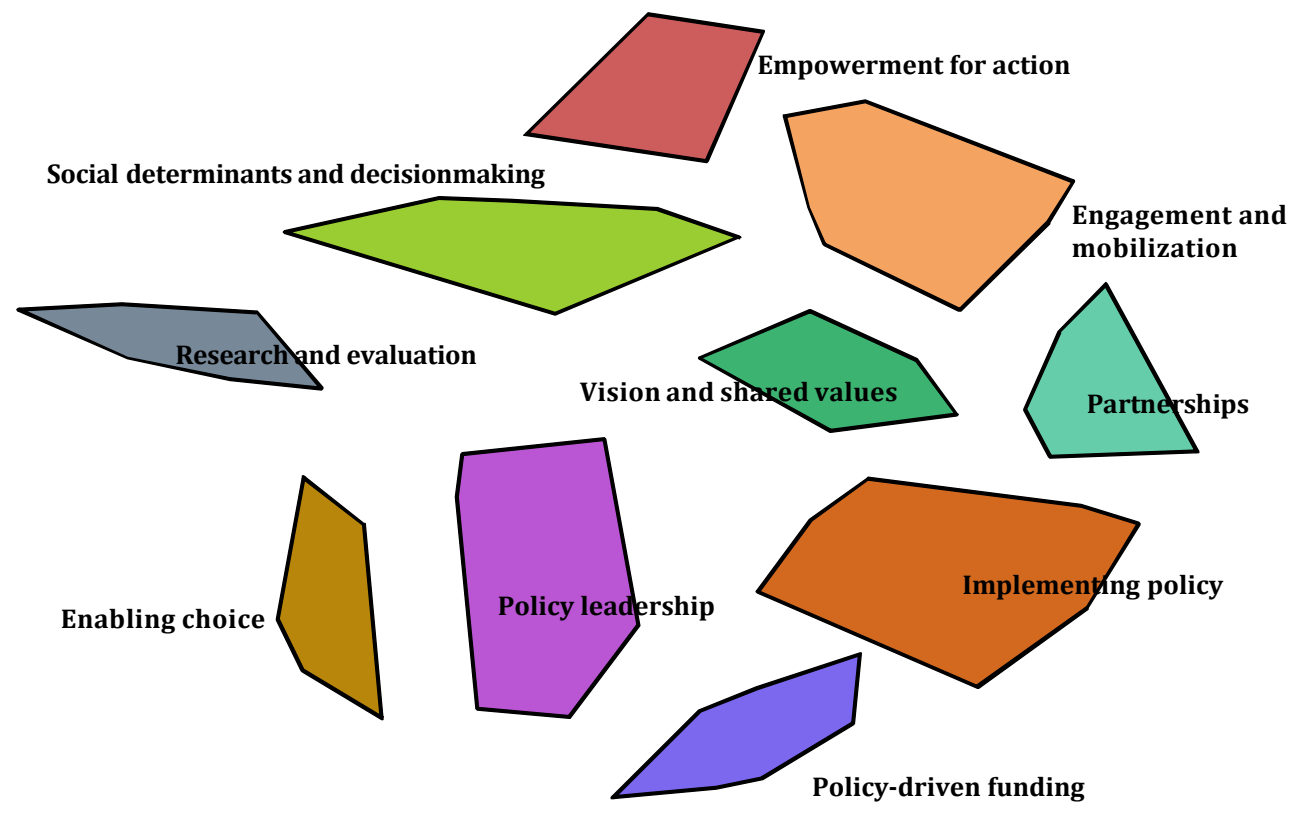

\section{Concept Mapping Phase Two}

The second phase of concept mapping was expanded to include a greater focus on sector analysis. This approach allowed the team to capture feedback from professionals, as well as those who serve specific needs in communities related to a Culture of Health. The Concept Systems team conducted concept mapping using professional association networks (e.g., social service providers, city planners) to bring together single-sector and mixed-sector groups. These groups explored the action framework and offered ideas for advancing a Culture of Health. This approach allowed the team to further vet measures that could be used by one or more sectors. While the report for this phase has not been finalized by Concept Systems, the early findings support some of the illustrative measures identified for a Culture of Health.

\section{Remaining Chapters}

The next chapters summarize the development of each action area and identify core drivers and illustrative measures. As noted earlier, this report summarizes the background review that informed the first phase of the Culture of Health effort. However, there are ongoing efforts to evaluate use of the action framework, progress in driver and measures areas, and use of new approaches and new methods to deepen implementation of the Culture of Health locally and nationally. For example, RWJF is working with other grantees and partners to facilitate aspects 
of measures development, including developing partnerships with leaders in big data/machine learning analysis to determine what measures can be derived from this mode. RWJF is also collaborating with key leaders in chronic disease burden measurement to help with localized assessment of a Culture of Health. Finally, RWJF, in partnership with RAND, will convene technical experts to further inform and refine Culture of Health measures. 


\section{Making Health a Shared Value}

This chapter provides an overview of the action area of making health a shared value. This action area emphasizes transforming individual and community-level mindsets and expectations regarding health and well-being to ensure that populations value and prioritize health and recognize the links between personal health decisions, community well-being investments, and community health outcomes. This chapter begins with the vision for the action area and how it is defined, the respective drivers and the arguments for those drivers, and the first set of Culture of Health measures, as well as gaps in measurement. In particular, we draw on findings from the environmental scan, the inputs from RWJF partners (e.g., ABCD, the meeting of cultural sociologists that we convened), and feedback from stakeholders.

\section{Envisioning and Defining This Action Area}

This action area focuses on the degree to which health is a shared value among individuals and the extent to which individuals feel a sense of interdependence. In this context, shared values mean that individuals and whole communities prioritize health and that health informs and drives local decisionmaking; that communities have high expectations for their environment, health system, and supporting services; and that people understand that their health influences and affects others, and vice versa. Accordingly, people are called to action to make decisions that not only promote their own health but also the health of those in their communities.

Shared values among individuals can influence shared values among organizations, so the process of making health a shared value has multiple levels. It includes relationships among neighbors and family members, among organizations, and between individuals and organizations. Making health a shared value does not mean that there is a single Culture of Health, but rather cultures of health characterized by some alignment in values or community expectations and actions in ways that promote health and well-being (e.g., culture defined by geography, ethnicity).

The research guiding this action area relies heavily upon concepts related to social networks, community resilience, well-being, behavioral economics, and asset-based community development. 


\section{Drivers in the Action Area of Making Health a Shared Value}

Three drivers are critical for ensuring that health is a shared value: mindset and expectations, sense of community, and civic engagement. The sentiment that a shared value of health (i.e., prioritizing health, thinking that it is something to be valued) was a prerequisite for building a Culture of Health was a theme common across the environmental scan, stakeholder input, and RWJF partners. To create a shared value of health, stakeholders and RWJF partners described the need to communicate clearly and improve general population understanding that health is a priority. Creating this awareness would then create the demand for healthy communities, leading to changes that begin to shift the momentum toward a Culture of Health. To fully capture the drivers in this area, we needed to ensure that we had drivers that targeted the understanding of the general population (i.e., mindset and expectations), as well as the ability of a community to mobilize or organize around a demand signal to create health communities (i.e., civic engagement) and/or to be influenced by a demand signal (i.e., membership and shared emotion connection created by a sense of community). As noted in Chapter Two, we winnowed the number of drivers down to these final three, using various forms of stakeholder and expert input.

\section{Mindset and Expectations}

This driver covers the perspectives and views that individuals, families, and organizations hold about health. It encompasses the factors that people indicate will contribute to a culture or cultures of health, the extent to which people and organizations prioritize health in their planning and processes, and general views of the influence of health decisions on community well-being.

Understanding where community members start in their mindset and expectations will inform where community engagement and information processes may need to start in order to catalyze community health action. For example, the W.K. Kellogg Foundation (2007) observed that collective leadership in a community can be achieved when residents are motivated by a common purpose and start to build relationships to co-construct their shared purpose and work. This attitude transformation has four stages: understanding the power of place, developing collective leadership, developing the individual, and making the change.

Building a clear case for the importance of health is intrinsic to a Culture of Health. Without this clarity, it is not only difficult to change attitudes about health but also challenging to galvanize a social movement to actively promote health. According to Swidler (1986), "values 
remain the major link between culture and action." As noted in Chapter One, culture is bounded, aligned, and shared. For that alignment to occur, populations and communities require a better understanding of health to frame community and national plans. This does not mean that the value proposition of health is exactly the same across cultures and communities. Rather, the value of health can honor diverse community members' perspectives, narratives, and cultural meanings of what constitutes health and integrate them into policies, practices, and strategies for improving health (Swidler, 1986).

Mindset and expectations may also be guided by such factors as feelings of altruism or compassion. Altruism and compassion link to the notion that health is interdependent and can inform people's attitudes about the health and welfare of their fellow community members (Maben, Cornwell, and Sweeney, 2010). Life meaning and individual sense of purpose, which typically inform notions of personal spirituality, may also drive a common community purpose for health (Baumeister, 1991). Without these emotional states, it may be difficult to activate community interest in and expectations about creating a shared, aligned Culture of Health.

Empowerment is also a part of the mindset and expectations for a Culture of Health. People and organizations must feel empowered in order to change their expectations of health and increase demand for better health. In both developed and developing countries, empowerment has led to better health outcomes. Laverack (2006) identified key factors for empowerment as (1) a sense of participation, (2) the presence of community-based organizations, (3) local leadership, (4) resource mobilization, (5) community activities that allow the asking of "why," and (6) constant assessment of problems within a community.

Finally, in the convening of cultural sociologists led by RWJF (described in Chapter Two), these research leaders noted that communicating about health is part of "social values." They noted that the cultural environment (e.g., how family is viewed, how tradition is viewed) supports how people feel about health as well, and that individuals live in many cultural spaces that influence their perspectives and orientation to health. As such, the factors that characterize cultural environment also influence attitudes about health.

Based on the literature review, several key factors influence mindset and expectations for health at the individual, organization/community, and decision environment levels. We summarize those in Table 5.1. 
Table 5.1. Factors Influencing Mindset and Expectations for Health

\begin{tabular}{|l|l|}
\hline Level & Factors \\
\hline Individual & - $\begin{array}{l}\text { Positive attitudes about health and well-being } \\
\text { Preference for engaging in healthy behavior }\end{array}$ \\
\hline Organization/community & - $\begin{array}{l}\text { Organizational prioritization of health and advocacy for health } \\
\text { Community norms, prioritization of health }\end{array}$ \\
\hline $\begin{array}{l}\text { Decision environment/ } \\
\text { policies }\end{array}$ & - $\begin{array}{l}\text { Policies that prioritize the value of community health } \\
\text { - Integration of information that demonstrates higher expectations for health as } \\
\text { part of local or national decisionmaking }\end{array}$ \\
\hline
\end{tabular}

\section{Sense of Community}

A strong sense of community is critical to advancing a Culture of Health for two reasons. First, communities that can connect and organize effectively are better able to identify needs and promote action. In short, "having a stake in the local community gives people self-respect and makes them feel better" (Morrow, 2001). Second, communities that are strongly connected and trust local structures, people, and processes can create a foundation that supports a culture (or cultures) of health and recognizes cultural values, norms, and traditions. A sense of community has been central to collective action work, and it will be key to advancing change and having communities own their health decisionmaking.

Given the links between sense of community and social connectedness, it is important to define the latter. Social connectedness refers to the personal (e.g., family, friend, neighbor) and professional (e.g., service provider, community leader) relationships among community residents. Relationships can vary in closeness (acquaintance versus close friend) and can be with individuals who are similar in status (i.e., horizontal or parallel) or with individuals of varying status and power (i.e., vertical or hierarchical). When residents have relationships with other members of their community, it increases their attachment to the community, access to real and perceived social support, and social capital (Kawachi, Subramanian, and Kim, 2008), as defined by the feelings of trust and norms of reciprocity that develop as a result of relationships (Putnam, 2000). This connectedness also promotes a sense of community, or the feeling of belonging that members have - that is, whether they matter to one another and to the group and whether they hold a shared faith that members' needs will be met through their commitment to be together (McMillan and Chavis, 1986). Individuals who live in communities with these characteristics 
have better psychological, physical, and behavioral health (Varda et al., 2009) and are fundamentally better able to thrive. In addition, people with a greater sense of community are more concerned with maintaining their connections to the community when stressful situations occur (Yong-Chan and Jinae, 2010).

Further, an understanding at the individual, organizational, and community levels that health is interdependent helps to support the creation of shared values and cohesion about health. If community members do not see their health as influencing the health and well-being of their colleagues, neighbors, and peers, they are less inclined to engage in health-promoting behavior for themselves and their communities. If this interdependence is framed in compelling ways, it may lead to increased demand for affordable and high-quality health care, neighborhoods that are healthy, and policies that promote health. At an organizational and community level, Kramer and Hogue (2009) define shared value as the policies and operating practices that enhance a company's competitiveness while advancing economic and social conditions in the communities in which it operates. Creating a shared value of health could employ a similar multilevel approach by identifying and expanding the connections between social and economic progress.

In summary, the factors in Table 5.2 influence sense of community. One might consider how these factors can be supported through intervention and policy and examine the association between relative sense of community and the comparative strength of a Culture of Health.

Table 5.2. Factors Influencing Sense of Community

\begin{tabular}{|l|l|}
\hline Level & Factors \\
\hline Individual & - $\begin{array}{l}\text { Views about value of collaborating with neighbors/community members, } \\
\text { reliance on neighbors for support }\end{array}$ \\
\hline Organization/community & - $\begin{array}{l}\text { Pngagement in social activities, participation in social organizations } \\
\text { ("community spirit") }\end{array}$ \\
\hline $\begin{array}{l}\text { Decision environment/ } \\
\text { policies }\end{array}$ & - Organizational collaboration for health and community development \\
\hline
\end{tabular}




\section{Civic Engagement}

Civic engagement processes in which community members feel that they can - and want to-influence change are important to creating shared values. Civic engagement is the process of "working to make a difference in the civic life of communities and developing the combination of knowledge, skills, values, and motivation to make that difference. It means promoting the quality of life in a community, through both political and non-political processes" (Ehrlich, 2000). A civically engaged person accepts moral and civic responsibility for himself or herself and recognizes his or her place in the larger social fabric. The person can accept social problems as partly his or her own and is able to understand larger social issues and take action when appropriate.

Civic engagement is a critical driver toward creating health demand and cultivating complementary plans and incentives that lead to shared results. If all parts of a community are not effectively engaged, equity may be compromised (Oakley, 1999). For example, how does social participation contribute to a community's ability to advance a Culture of Health? The scale and scope of social participation (size, composition, sources of support) and the values, norms, and cultural expectations it represents are important predictors of the impact that social and community-based organizations will have on promoting citizen voice, policymaking, and service provision. The legal and political context also influence both the structure and values of these organizations and mediate the organization's impact (Anheier, 2004).

Another component of civic engagement is awareness of the health assets that exist in a community, which can move the needle from "preventing disease" to "promoting a Culture of Health." Civically engaged communities can begin to move to asset-based models for health and determine ways to build on those strengths (Dorfman, 1998). Health assets include the presence of active community-based organizations or social connectedness among residents. As noted earlier, social capital has also been considered a key asset because it drives social influence in positive and negative ways (Portes, 1998). While little research has linked asset mapping to a Culture of Health, similar associations have been articulated in the related fields of community resilience and community well-being (Norris et al., 2008).

Civic engagement also lies in the ability of businesses, community organizations, and other anchor institutions to support that community engagement. The Initiative for a Competitive Inner City noted that understanding and measuring the shared value of anchor institution strategies- 
via the positive impact on the community and the positive returns to the anchor - might strengthen the investment that these organizations make to engender civic engagement (Initiative for a Competitive Inner City, 2011).

Reviews of civic engagement strategies have revealed the following qualities of what contribute to success: a commonly accepted broker or keeper of the vision in a community or organization; clear and well-defined roles and responsibilities of civic institutions; and the leadership, staff capacity, and ability to turn data into usable information across the community for civic health promotion or broader advocacy (Chavis, 2006).

Given these findings, civic engagement is influenced by the factors in Table 5.3. These factors provide potential levels of influence or change that can affect whether civic engagement for health and well-being decisionmaking is achieved.

\section{Table 5.3. Factors Influencing Civic Engagement}

\begin{tabular}{|c|c|}
\hline Level & Factors \\
\hline Individual & $\begin{array}{l}\text { - Interest in civic participation or advocacy } \\
\text { - } \quad \text { Sense that civic participation matters and is valued }\end{array}$ \\
\hline Organization/community & $\begin{array}{l}\text { - Organizational support of civic engagement activities } \\
\text { - } \quad \text { Community orientation to civic participation in decisionmaking } \\
\text { - Knowledge of community assets }\end{array}$ \\
\hline $\begin{array}{l}\text { Decision environment/ } \\
\text { policies }\end{array}$ & $\begin{array}{l}\text { - } \quad \text { Policies that require or at least support civic participation in decisions } \\
\text { - Governance structures that are inclusive of civic engagement across } \\
\text { individuals and organizations }\end{array}$ \\
\hline
\end{tabular}

\section{Measures}

Several measures were identified as illustrative of each driver. We describe each measure in further detail below, and then summarize them in Table 5.4. For a summary of the broader measures selection process, see Chapter Three. Note that more detail on how each measure is operationalized is provided on the Culture of Health website (see www.cultureofhealth.org).

\section{Mindset and Expectations Measures}

We identified three measures to capture changes in mindset and expectations regarding a Culture of Health.

Proportion of Twitter mentions discussing health promotion and well-being to Twitter mentions discussing acute medical care. This measure takes advantage of social media to explore 
attitudes related to health and well-being. The amount of discussion (e.g., number of Twitter mentions) that Americans have about wellness or well-being-oriented activities, such as healthy eating, physical activity, or stress management, offers one insight into public sentiment about a Culture of Health. This measure addresses several aspects of this driver, mainly via identifying health attitudes and how those are being expressed online. This analysis demonstrates the varying sense of community or civic engagement around specific topics beyond wellness or acute care broadly, such as physical activity and healthy eating.

Health-related discourse generally reflects predominant attitudes and norms and the public's understanding about health issues and their potential influence on public perceptions, attitudes, and social norms (Lawrence, 2004; Lupton, 1992; Van Dijk, 1993). Further, performing healthpromoting behaviors is influenced by attitudes and social norms about prevention and wellness (Ajzen and Timko, 1986). Extensive research in the general area of social media analysis suggests that relative frequencies of various categories of word usage can illustrate health attitudes, behaviors, and outcomes (Chung and Pennebaker, 2007; Eichstaedt et al., 2015; Mitchell et al., 2013; Schwartz et al., 2014). More specifically, RAND pilot research on these topics has shown that these indicators vary by geographic region, demographics, and social influence.

Social media research is still relatively nascent, but researchers in this area assert that frequent usage is related to general interest in a topic (Chung, 2014; Chung and Pennebaker, 2007; Mitchell et al., 2013), particularly when considering text and sentiment analysis.

Percentage of adults in strong agreement that health is influenced by peers, neighborhood, and the broader community. Historically, we have not elevated the importance of health relationships. This measure uniquely demonstrates whether and how Americans feel that their health is related to that of their peers, neighbors, and other community members. This relates to a Culture of Health because culture is not created individually but interdependently. If people prioritize and value health, they will expect that their community peers will not only do the same but will also influence the actions of that community to promote health. Additionally, as summarized earlier in this chapter, understanding where community members start in their attitudes and expectations will inform where to catalyze community health action. Altruism and compassion link back to the notion that health is interdependent (Maben, Cornwell, and Sweeney, 2010), and factors that influence a community purpose for health (Baumeister, 1991) 
require a network of connections to move toward shared understanding. Note that, as described in previous chapters, this and the next measure were developed specifically as part of this effort and fielded in the RWJF Survey of National Health Attitudes.

Percentage of adults interested in how their community invests in well-being, signaling a broader expectation for well-being. Well-being comprises both individual and community action areas, as well as subjective and objective measurement. There has been more focus in research on individual well-being, defined as the extent to which people experience happiness and satisfaction and are realizing their full potential. Yet, aspects of community well-beingincluding the levels of community health, economic resilience, educational capacity, and environmental adaptation (Diener and Chan, 2011) — are key to a Culture of Health. Measuring well-being and understanding the drivers of well-being and how they interact can help to create a more holistic and informed policymaking approach that influences whether community wellbeing investments are made. Emerging evidence suggests that well-being and happiness are related to many community outcomes of health and social development, including stronger marriages, increased educational and work-related accomplishments, community involvement, and better health outcomes (Howell, Kern, and Lyubomirsky, 2007; Lyubomirsky, Sheldon, and Schkade, 2005; Pressman and Cohen, 2005). As such, community views on well-being investments, including how government chooses to increase well-being, can enhance programs that support the health and well-being of community members, who, in turn, are more productive and supportive of their community.

\section{Sense of Community Measures}

We identified two measures to capture a sense of community that can help create the conditions for a shared value of health.

Emotional connection to and membership in community. A strong sense of community is critical for advancing a Culture of Health. As noted earlier, communities that can organize and connect effectively are better able to identify needs and promote action. The Sense of Community Index (SCI) demonstrates how people feel about their community — whether they believe that they belong and can count on their peers. The SCI incorporates McMillan and Chavis' (1986) elements of a sense of community, including shared emotional connections, membership, influence, and whether the community is meeting residents' needs. For this 
measure, we focus on two subscales. The first is emotional connection to community, such as connection to other community members and expected tenure in the community. The second is the membership subscale, which includes sentiments of trust in fellow community members. Taken together, these two subscales offer keen insight into whether a community has the foundational elements needed for creating shared value and meaning regarding health.

Percentage of adults noting that they have adequate social support from their partner, family, and friends. Positive social support is a critical component of making health a shared value. It can have behavioral, psychosocial, and physiological influences on individuals (Umberson and Montez, 2010) and plays a large role in a person's ability to make healthier choices. For example, emotionally, social support and social integration can serve as a source of motivation and social pressure to engage in healthy behaviors (S. Cohen, 2004). Physically, social support systems can provide rides to medical appointments or access to healthier meals.

Social support also can help protect community members from negative health outcomes and influences. Having a spouse, family member, or friend on whom one can rely during difficult times can help individuals come through these times with better health and with more resilience. Furthermore, individuals who are isolated from social supports have been shown to have worse health overall (more likely to develop disease and die). Social support is an indicator that a community has an important asset (i.e., social relationships) needed to create a shared value of health. When residents have relationships with other members of their community, it increases

their attachment to the community, access to real and perceived social support, and social capital. The benefits of social capital, defined as the capacity to secure benefits through membership in social networks, may include the ability of community to leverage health assets to actively enhance health on an ongoing and continuous basis (Kawachi et al., 1997).

Traditionally, the Behavior Risk Factor Survey, conducted by the Centers for Disease Control and Prevention (CDC), captures experience of adequate social support. However, the item is not currently systematically assessed across all states, so it was implemented in the RWJF National Survey of Health Attitudes.

\section{Civic Engagement Measures}

The team identified two measures as indicators of civic engagement. However, these measures assess neither the full range of civic engagement processes, including local voting and 
community organizing, nor the quality or impact of engagement. Those activities will be better captured using community-level data. Instead, the two measures noted here illustrate national behaviors related to civic participation.

Percentage of eligible voters who vote in general elections. Voter turnout is an indicator of strong citizen engagement in important issues in the nation and in a community. High voter participation has been a key feature in communities where residents share the belief that they can influence important issues and where inequality and health disparities are minimized. Furthermore, voting demonstrates that members feel connected to their countries and communities and care about influencing change. This is another hallmark that a community or state may have a strong foundation to create a shared value of health. Voting allows citizens in different communities across the United States to influence the political issues that they care about (Verba, Nie, and Kim, 1978). Emerging research is now linking voting and mental health. In 2001, Blakely noted that disparities in political participation across socioeconomic status affect health. States with the greatest inequality in voting and income also have the lowest selfrated health. Finally, Crompton and Kasser (2010) noted that being politically active is linked with greater well-being and life satisfaction.

Percentage of adults and young people reporting volunteering (national and by state). Volunteering is a critical hallmark of citizen engagement. It has been key to supporting the resilience of a community during emergencies because communities that can access volunteers as part of local capacity are better able to respond and recover. Furthermore, volunteering demonstrates that community members care about community outcomes and want to effect change.

Studies now show that those who volunteer feel better, both physically and emotionally. Some surveys (see UnitedHealth Group, 2013) note that more than three-fourths of those who volunteer indicate that it makes them feel healthier, and nearly 80 percent reported that volunteering helps to reduce stress levels. Herzog et al. (1998) and Harlow and Cantor (1996) noted that participation in community service was strongly associated with life satisfaction among older adults. Arnstein et al. (2002) noted that individuals with disability or chronic pain were able to mitigate their depression and intensity when serving as volunteers. The Corporation for National and Community Service and local organizations have often increased volunteering through employer engagement, incentives, and working with neighborhood groups. Programs 
that support volunteering and reward that behavior in some way can motivate changes in overall volunteer rates.

Table 5.4 summarizes the measures described in the preceding sections. As noted, these measures illustrate the three drivers for this action area.

Table 5.4. Making Health a Shared Value: Measures by Driver

\begin{tabular}{|c|c|c|}
\hline Driver & Measure & $\begin{array}{l}\text { What It Means to the Action Area/ } \\
\text { Culture of Health Overall }\end{array}$ \\
\hline $\begin{array}{l}\text { Mindset and } \\
\text { expectations }\end{array}$ & $\begin{array}{l}\text { Proportion of Twitter } \\
\text { mentions discussing health } \\
\text { promotion and well-being } \\
\text { to Twitter mentions } \\
\text { discussing acute medical } \\
\text { care }\end{array}$ & $\begin{array}{l}\text { Indicates shift in consumer behavior and } \\
\text { sentiment around health promotion, not just } \\
\text { treatment of disease }\end{array}$ \\
\hline $\begin{array}{l}\text { Mindset and } \\
\text { expectations }\end{array}$ & $\begin{array}{l}\text { Percentage of adults in } \\
\text { strong agreement that their } \\
\text { health is influenced by } \\
\text { peers, neighborhood, and } \\
\text { the broader community }\end{array}$ & $\begin{array}{l}\text { Indicates the extent to which there is } \\
\text { understanding that health is interdependent and } \\
\text { that individual behaviors influence community } \\
\text { well-being, and vice versa }\end{array}$ \\
\hline $\begin{array}{l}\text { Mindset and } \\
\text { expectations }\end{array}$ & $\begin{array}{l}\text { Percentage of adults } \\
\text { interested in how their } \\
\text { community invests in well- } \\
\text { being }\end{array}$ & $\begin{array}{l}\text { Offers insight into individual interest and } \\
\text { priority placed on investments to advance a } \\
\text { Culture of Health and signals a broader } \\
\text { expectation for well-being }\end{array}$ \\
\hline Sense of community & $\begin{array}{l}\text { Aggregate score on two } \\
\text { subscales of Sense of } \\
\text { Community Index: } \\
\text { emotional connection (to } \\
\text { community) and } \\
\text { membership (sense of } \\
\text { belonging to community) }\end{array}$ & $\begin{array}{l}\text { Conveys the relative sense of cohesion, which is } \\
\text { integral to advancing a Culture of Health and } \\
\text { shared engagement and values }\end{array}$ \\
\hline Sense of community & $\begin{array}{l}\text { Percentage of adults noting } \\
\text { that they have adequate } \\
\text { social support from partner, } \\
\text { family, and friends }\end{array}$ & $\begin{array}{l}\text { Indicates both the sense of connectedness } \\
\text { around health and well-being and the extent to } \\
\text { which community members are providing } \\
\text { supportive, healthy environments }\end{array}$ \\
\hline Civic engagement & $\begin{array}{l}\text { Percentage of eligible } \\
\text { voters who reported voting } \\
\text { in general election }\end{array}$ & $\begin{array}{l}\text { Offers one indicator of civic participation, which } \\
\text { is a key indicator of whether a Culture of Health } \\
\text { can thrive and enable community members to } \\
\text { work collectively toward health and well-being }\end{array}$ \\
\hline Civic engagement & $\begin{array}{l}\text { Percentage of adults and } \\
\text { young people who report } \\
\text { volunteering }\end{array}$ & $\begin{array}{l}\text { Offers two indicators: another aspect of civic } \\
\text { participation and a measure of pro-social, } \\
\text { altruistic behavior-key in a Culture of Health }\end{array}$ \\
\hline
\end{tabular}




\section{Limitations}

Although these measures are feasible options for assessing some aspects of the development of shared values for health, there are some gaps in what is measurable now and areas where measurement needs to be further developed and advanced.

There are few measures that fully assess community collaboration for health and the voice of traditionally marginalized populations in health. While the measures that are available for sense of community and civic engagement begin to provide insight into such areas as general engagement in social and political groups, there are limitations in current measurement to determine the extent or nature of the level of civic participation, as well as the quality of how community members come together to create shared community values. For example, national survey measures may not fully capture the civic engagement processes across groups in a community, particularly those whose voice in civic decisionmaking tends to be limited. It will be important for Culture of Health measurement to identify unique measures and data collection modes to obtain information from these often disenfranchised groups. In addition, aside from survey measures about perceived social support and neighborhood cohesion, very little data exist on how a sense of community is cultivated or fostered or whether and how it differs by region.

There are few measures on the activation of community processes to advance health. "We're all in this together" is a key principle for RWJF's Culture of Health effort, but to achieve this state, it is important to have measures that assess progress toward community members experiencing and elevating the discussion of health collectively. Toward that end, we will explore measures to identify points of transition or change in a community, such as when general civic engagement results in a true sense of community about health and in full participation in local or national health decisionmaking. Culture of Health measures may need to change over time to determine progress toward greater community involvement in health promotion. For example, the first measure may be about general civic participation, but if progress is made, later measures should assess civic leadership to plan for health or shared accountability for health decisions.

Finally, there are few measures on broad community investment in health and well-being. Given that Culture of Health outcomes are not simply the absence of disease and the efficiencies gained for the health care system but, rather, broader well-being, Culture of Health measures should include items that track investment in community resources promoting well-being. This 
may include determining the extent to which a community has activities and practices that promote well-being or the extent to which individuals understand the personal inputs required not to merely stay healthy, but to thrive and flourish. To date, few national measures exist to track these investments, and while more information may exist at the community level, these data are not systematically collected and used. 


\section{Fostering Cross-Sector Collaboration to Improve Well-Being}

This chapter provides an overview of the action area related to fostering cross-sector collaboration to improve well-being. Over the past decade, U.S. population health improvement efforts increasingly have focused on strengthening relationships between health care and public health sectors, as well as between these sectors and others in which health is seen as a secondary mission (e.g., education, housing, social services). Developing partnerships between health sectors and those not traditionally considered to be health-related (e.g., business, economic development, media, law enforcement) is a newer approach. The shift to include these nontraditional sectors has been driven by mounting evidence regarding the social, policy, and environmental determinants that drive health and well-being at organizational, community, and national levels.

In this chapter, we discuss cross-sector partnerships, collaborations, and investments to improve health and well-being, building on the initiatives to expand "health in all policies" (Leppo et al., 2013). We draw on the study team's broad environmental scan of cross-sector collaborations for health and well-being and several complementary exploratory projects commissioned by RWJF, including (1) a concept mapping (Trochim and Linton, 1986) community development project conducted by Concept Systems and (2) projects examining current and potential roles of the business sector and affordable care organizational efforts conducted by HERO (2015a) and HRET (2014), respectively.

\section{Envisioning and Defining This Action Area}

The purpose of cross-sector collaboration to improve health and well-being is to optimize the contributions of multiple sectors by linking more traditionally health-focused sectors (e.g., public health, health care, social services) with sectors whose contributions to health and well-being have come into focus more recently (e.g., business, education, faith, housing, law, transportation, zoning). The quality and extent of these partnerships and the nature and extent of investments in these partnerships should be considered. Cross-sector collaboration holds unmet potential as an engine for aligning the assets, policies, and practices of multiple sectors to (1) improve the health and well-being of organizations, communities, and the nation; (2) improve health equity across 
diverse racial/ethnic and socioeconomic groups and populations; (3) help entities across sectors achieve their individual goals more effectively; and (4) find novel (and possibly more effective) strategies for fostering population health and well-being. Examples of such cross-sector collaborations already exist, including the Fitness Improvement Training Zones initiative sponsored by the East Palo Alto Police Department, which identifies the most dangerous neighborhoods through crime data and then implements health-related programs in public spaces so that residents can reclaim areas that had been overtaken by gang members.

In a review of health in all policies, a report by the Finnish government (Leppo et al., 2013) cited numerous examples of how this approach has improved health and well-being. For example, the Natural Resources Defense Council (NRDC) spent years trying to raise awareness with the public and the government about key environmental issues. In 2007, NRDC partnered with legal experts to win a historic Supreme Court ruling that classified global warming emissions as "pollutants" under the Clean Air Act and granted authority and responsibility for regulating those pollutants to the Environmental Protection Agency.

Another key area that can be addressed by health in all policies is worker health. Conditions of employment may be a powerful determinant of health for the more than 3 billion people globally who spend one-third of their adult life working. Employment conditions vary greatly across countries, and work policies that target health can make a big difference to workers. For example, the State Council of China's National Occupational Disease Control Programme (2009-2015) has objectives to increase occupational health and safety measures and coverage significantly across workplaces in China. Specific targets include the monitoring of hazardous exposures in 70 percent of workplaces, monitoring the health of 60 percent of workers in hazardous jobs, and providing 90-percent coverage of accident insurance for workers.

In another example of the impact of employers on worker health, 84 percent of 583 large U.S. employers offered on-site influenza vaccination to employees, which may improve the low influenza vaccination rates among working-age individuals (Graves et al., 2014). Additionally, a U.S. trial of an intervention of best practices to prevent chronic disease in the workplace showed that employers in the intervention demonstrated greater improvement in implementing best practices and communications than those in the control group (Hannon et al., 2012).

There are numerous examples of cross-sectoral initiatives that are centered in nontraditional health sectors but are influencing population health and well-being outcomes. For example, 
Acevedo-Garcia et al. (2004) and Thomson, Petticrew, and Douglas (2003) demonstrated the multiple health benefits of investments in housing. Research has long exposed the negative effects of poor housing on early childhood development, injuries, mental health, and chronic disease incidence and management. The primary method of combating these negative outcomes has been through enforcing housing codes, creating healthy homes, and providing collaboration and cross-sector planning (Krieger and Higgins, 2002). Housing unit location and quality can significantly impact child stress and adult health outcomes.

Similarly, Littman (2010) evaluated how public transportation affects health directly and indirectly, using such tools as the Active Transport Quantification Tool and the Physical Inactivity Cost Calculator. In the business sector, Chu et al. (2000) and Harris et al. (2014) have documented the pervasive and beneficial effects of workplace health promotion programs on employee health and well-being and on the health of the surrounding community, and there is growing evidence that these interventions improve business profits (Pronk et al., 2015). Similarly, a comprehensive review by Brownson et al. (2006) found that collaborations among urban planning, transportation policy, and school-based physical education can change health by creating environments and decision prompts (e.g., signs next to elevators, labeling on buses) that promote physical activity and help to prevent or reduce obesity at the population level.

\section{Drivers in the Action Area of Fostering Cross-Sector Collaboration to Improve Well-Being}

The view that cross-sector collaboration must be augmented and strengthened was a critical theme common across the environmental scan, stakeholder input, and RWJF partners.

Three drivers are critical for ensuring that cross-sector collaboration is achieved: number and quality of partnerships, resource investments across sectors, and policies that support collaboration. They were developed based on a proposed theory of change in which cross-sector partnerships are initiated in order to expand the impact of health care sector efforts. However, significant expansion of cross-sector collaborations will need supports, such as policies and investment in these emerging structures. Public policies supporting cross-sector collaborations are ideally tailored to the needs of the community, which can be large or small jurisdictions, and should be based on evidence. Investments in such collaboration need similar guidance. As jurisdictions begin to implement their own Culture of Health efforts, data collected as part of 
those efforts (e.g., sentinel surveillance communities) can be used to inform local policy development and investment.

Efforts around these drivers were laid out in a three-part sequence to enable sectors to know their roles and contributions, work effectively together, and have the resources and policies to support ongoing collaboration. For number and quality of partnerships, we asserted that in order to strengthen cross-sector collaboration, we should focus on the number and extent of collaborative partnerships between health and non-health sectors (Baezconde-Garbanati et al., 2006; Pant et al., 2008). In short, it is important both to enumerate specific partnerships between the health sector and other sectors and to document the quality of these partnerships. This driver would also focus on delineating what contributions each sector can bring to a Culture of Health. The second driver focuses on the resource investments across sectors. This includes resources committed to drive partnership activities that produce positive health and well-being outcomes (Wholey, Gregg, and Moscovice, 2009). The third driver examines policy supports for productive cross-sector collaborations, ensuring that there is a structure that will maintain and develop partnerships over time (Barrett et al., 2005; Ribisl et al., 2003).

\section{Number and Quality of Partnerships}

Cross-sector partnerships that influence health and well-being are proliferating. Several organizations (e.g., National Association of County and City Health Officials, American Heart Association) track and report information on the occurrence and nature of partnerships with health-sector organizations. These data have been used to assess the number of sectors to which health agencies are connected in communities (Willard et al., 2012). The literature also provides ample evidence that government and nongovernmental organizations working together is a key factor in building a Culture of Health. Communities with strong cross-sector integration and engagement are better equipped to support a community's response to different types of stressors (Baezconde-Garbanati et al., 2006; Pant et al., 2008), in part because they can engage a variety of nongovernmental organizations and local groups with vital assets (Stewart, Kolluru, and Smith, 2009). The emphasis of this driver is to concretize such partnerships (with funding when available), rather than maintaining informal networks, so that they can be counted and evaluated (Chandra et al., 2013). 
Bringing together entities across sectors increases the likelihood that policies and practices that provide access to health and well-being resources (e.g., green spaces) are equitably distributed across the community and responsive to population diversity and changing community demographics. For example, growing evidence that the built environment affects physical activity and health (Frank and Kavage, 2009; Handy et al., 2002) has inspired public health experts, urban planners, architects, and public- and private-sector decisionmakers to work together to develop and implement healthy community design principles (Dannenberg et al., 2003).

The mere existence of partnerships does not guarantee that the activities generated will improve community health outcomes. New models of effective partnerships (e.g., RWJF's County Health Rankings \& Roadmap) provide guidelines for assessing the strength and quality of partnerships along a continuum from minimal interaction (e.g., sharing information at meetings) to coordinated activities that involve sharing resources to achieve a specific health outcome. Partnership efforts range from simply updating each partner on activities (lower quality) to collaborations in which partners share significant time and resources to achieve common goals (higher quality). As a result, the contributions of cross-sector partnerships to health and well-being depend on the quality of the partnership.

Community-wide expectations and incentives are important for sustaining cross-sector collaborations for health. As community stakeholders determine each sector's role in supporting broader and more integrated health planning and decisionmaking, shared accountability and incentive structures that value these collaborations are an essential driver in whether partnerships are robust and how they can persist over years. Without that attention, partnerships that are created based on grant guidance or initial interest may struggle to achieve outcomes without long-term sustainability (Zahner and Corrado, 2004). An emerging alternative model that should be considered is policy that designates a "backbone organization" for collaborative health initiatives. Backbone organizations maximize the collective impact of cross-sector collaboration by developing a guided vision and strategy, supporting aligned activities, establishing shared measurement practices, engaging the public, advancing policy, and mobilizing funding (Turner et al., 2012). Local health departments are a logical choice to serve as backbone organizations to spearhead cross-sector health efforts in communities. 
Another driver of quality collaborations is having members who work well together with shared goals for far-reaching community benefits that can strengthen the community's capacity to address health issues. For example, the Community Preventive Services Task Force recommends the implementation and maintenance of school-based health centers (SBHCs) in low-income communities based on evidence of their effectiveness in improving educational and health outcomes for children. However, policymakers are now exploring how SBHCs can serve not just students but also their families by enhancing actual and perceived access to resources for health and well-being and opportunities for personal engagement in community transformation.

Factors that are important to high-quality, productive partnerships include having a clearly defined shared goal; high relevance, legitimacy, or credibility among partnership organizations; and mutual respect, trust, and shared commitment (Kapucu, Arslan, and Demiroz, 2010; Petersen et al., 2005). There are many examples that illustrate the impact of strong partnerships. Green and Klein (2011) reviewed the effectiveness of the Columbus Healthy Places program, which integrates or promotes active transportation characteristics in community design. The study identified qualities that contribute to success in multi-sector collaborations, including strong relationships with partner agencies, program maintenance through regular contact with partner agencies, and active defense of the public health role in urban development. Three studies led by Lasker (Lasker and Weiss, 2003a, 2003b; Lasker, Weiss, and Miller, 2001) found that building relationships among partners is the most challenging aspect of multi-sector collaboration, and that leadership is particularly important for creating partnership synergy. Similarly, the community engagement model, at the core of asset-based community development, has proven more effective than the lead agency model (O’Mara-Eves et al., 2013). Further, a high-quality collaboration is one that addresses complex problems and engages stakeholders throughout the process, in part by providing feedback on progress toward shared goals. Data systems at the city, county, state, and national levels inform efforts to improve population health across multiple sectors (e.g., interoperable data systems across sectors that can track outcomes over time and enhance the longevity and robustness of cross-sectoral collaborations).

Based on the research, Table 6.1 presents key factors that shape partnership development and quality across levels and domains of influence. 
Table 6.1. Factors Influencing Partnership Development and Quality

\begin{tabular}{|l|l|}
\hline Level & Factors \\
\hline Individual & $\bullet \quad$ Individual leadership styles \\
\hline Organization/community & $\bullet \quad$ Organizational reputation, history of collaboration \\
& $\bullet \quad$ Resource quality and need from each organization \\
& $\bullet \quad$ Trust in organization role \\
\hline $\begin{array}{l}\text { Decision environment/ } \\
\text { policies }\end{array}$ & $\bullet \quad$ Policies that evaluate/require specific collaboration quality \\
\hline
\end{tabular}

\section{Resource Investments Across Sectors}

Community investments in cross-sector initiatives with a health focus are important to track because the quality and nature of collaborations tend to relate closely to the magnitude of funds and resources invested in them. Similarly, resource investments indicate the value placed on cross-sector collaboration for health and well-being. Investments can grow as new evidence of the impact of cross-sector collaborations becomes available.

There are myriad ways in which entities in non-health sectors directly affect the health of individuals through investment (Adler and Newman, 2002; Stoto, Abel, and Dievler, 1996). Education-sector investments in such programs as the National School Lunch Program have a direct impact on children's health through improved nutrition (USDA Food and Nutrition Service, 2013a, 2013b). Investments in urban planning can improve residents' access to safe places to walk, bike, and play, as well as to health care centers, healthy food options, and healthrelated social services. Workplace health promotion programs provide resources and opportunity to improve diet, physical activity, and routine preventive screening and care (Roussos and Fawcett, 2000).

In addition to these investments, non-health sectors may invest resources that indirectly influence health. For example, communities may decide to improve public transportation to relieve traffic congestion and lower commuting times. This decision may include little to no collaboration with health sector entities in the planning phases, yet there are many opportunities for the health and transportation sectors to work together to promote the health of individuals (Sallis et al., 2004). For example, health and transportation providers could collaborate on education campaigns about the health and employment benefits of improved public 
transportation. They could also collaborate on research to identify the direct and indirect health benefits of public transportation on communities and the mechanisms of those benefits (e.g., civic engagement, access to affordable housing, community action groups and organizations, faith-based groups). The U.S. Department of Transportation and CDC have developed the Transportation and Health Tool to help communities better understand the health impacts of transportation systems (U.S. Department of Transportation, 2015).

Before sectors can invest, however, they must understand their roles and responsibilities. Cross-sector awareness of how each sector contributes to health is critical. Without clarity on roles, responsibilities, and expectations, it is difficult to determine how each sector (e.g., housing, childhood development) can best support the development or production of health (Wholey, Gregg, and Moscovice, 2009). The partnership process refers to the method in which cross-sector entities understand and define their contribution to health promotion, work within and across sectors to coordinate health policy development and national and community action, and align activities to meet sector-specific and shared outcomes for optimal health (Lasker and Weiss, 2003a, 2003b). Without purposive cross-sector engagement, health strategies and health promotion efforts could ignore the potential contributions of other sectors, potentially resulting in misaligned or poorly coordinated health promotion activities within communities.

Based on the research, Table 6.2 shares key factors that shape resource investments, across levels of influence:

Table 6.2. Factors Influencing Resource Investments

\begin{tabular}{|c|c|}
\hline Level & Factors \\
\hline Individual & - Expectations that sectors are contributing to health \\
\hline Organization/community & $\begin{array}{l}\text { - Sector contribution level and type, as well as link to health } \\
\text { - Sector interests and prioritization of health outcomes (for employees, } \\
\text { constituents, and community members) }\end{array}$ \\
\hline $\begin{array}{l}\text { Decision environment/ } \\
\text { policies }\end{array}$ & $\begin{array}{l}\text { - Incentives or rewards for sector investment in health } \\
\text { - } \quad \text { Policies that outline sector investment strategies }\end{array}$ \\
\hline
\end{tabular}

\section{Policies That Reflect and Encourage Collaboration Across Sectors}

Public policies at the local, state, and federal levels can play a role by encouraging, incentivizing, or sustaining collaborations across sectors that contribute to health. This third 
driver represents an important indicator of progress toward stronger cross-sector collaboration for well-being. Public policy can also be used to raise support for and awareness of the impact of different sectors on health and the capacity of those sectors to improve health (Lasker and Weiss, 2003a).

Several recent policy efforts support cross-sector collaboration between health and other sectors. For example, the Affordable Care Act (ACA) created new opportunities and incentives for hospital organizations, numerous governmental public health agencies, and other stakeholders to accelerate community health improvement by conducting triennial CHNAs and adopting related implementation strategies to address priority health needs (CDC, 2013). Under the ACA, hospital organizations satisfy their annual community benefit obligation by meeting those new requirements. CHNAs have guided the development of cross-sector collaborations between hospitals and with local public health agencies not only to conduct CHNAs (e.g., assessment, planning, investment, implementation, and evaluation) but also to launch promising community-wide programs designed to address the health needs of high-risk populations and to support research on system partnerships. In its analysis for the RWJF effort, HRET noted that ACA-guided activity at multiple levels of user engagement (e.g., at the patient/family, health care team, organization, and community levels) by different health systems resulted in better patient outcomes, improved consumer satisfaction, and higher return on health care investment (see the summary of HERO work in Chapter Two and HERO, 2015b; note that the HERO report is not yet publicly available as of February 2016).

There are many other examples of how sectors are using organizational policy to advance health, particularly in measurement to support quality improvement in health management programs (Barrett et al., 2005; Ribisl et al., 2003). For example, in the business community, HERO uses a scorecard to offer employers feedback on best practices and ways to improve health management programs in their corporations and in the communities where their employees live. The Dow Chemical Company uses a similar approach to guide health and safety for its employees.

Given that this driver is principally about the policy environment (one of the levels in the factors table), we do not provide a table of the factors that shape those policies across all three levels as we have in the preceding sections. 


\section{Measures}

The following measures were identified as illustrative of each driver. We describe each measure in further detail below and then summarize in Table 6.3.

\section{Number and Quality of Partnerships Measures}

We identified three measures to capture the number and quality of partnerships.

Percentage of local health departments that collaborated with community organizations in at least four public health program areas during the past year. Community organizations (and, in this case, those whose missions do not focus specifically on health care) can serve as a gateway to communicating with otherwise hard-to-reach and vulnerable populations (e.g., populations that are non-English speaking, homeless, undocumented). Local health departments (LHDs) that partner with key community organizations can leverage the assets, activities, language skills, and cultural competence of those organizations to build trust and generate change in target communities. Measures of collaborations between LHDs and such community organizations reflect the extent to which LHDs in the United States are working to benefit hard-to-reach populations, especially since these groups very often have the lowest levels of health and wellbeing. Results from this measure can help LHDs understand their gaps with both connected communities and services for those communities.

A key role of a local health department is to provide leadership through partnerships to leverage the local public health system (NACCHO, 2009). Especially in a Culture of Health, where fostering collaboration is highlighted, LHDs are increasingly taking on the role of a backbone organization for collaborative health initiatives. As noted earlier, backbone organizations maximize the collective impact of cross-sector collaboration (Turner et al., 2012). As many LHDs shift to this model, this measure seeks to quantify the number of LHDs that collaborate with community groups across multiple health areas.

Although data exist over multiple years, this measure has not been utilized for trend analyses. However, the National Association of County and City Health Officials' (NACCHO's) 2008 National Profile of Local Health Departments (NACCHO, 2009) shows that LHD collaborations vary by location and by sector. Data analyses show that addressing differences in capacity can change the number and types of partnerships that are created. Additionally, the 2013 NACCHO profile (NACCHO, 2014) shows variability in collaboration among program areas (e.g., 
emergency preparedness, tobacco, food safety), indicating that efforts in specific program areas influence the presence of collaborations in those areas. Finally, the 2013 NACCHO profile (NACCHO, 2014) also indicates that LHDs exist along a spectrum of collaboration in different areas (from no partnership to coordination to collaboration), indicating that interim progress for this measure can be tracked as well.

Number of school-based health centers that provide primary care. SBHCs - partnerships between the education and health sectors - are key to improving primary care and health outcomes for children and families with limited resources. For children living in poverty, SBHCs are an important link to routine health services, including preventive services (e.g., vaccinations) and health education. SBHCs provide health services to $\mathrm{K}-12$ students and may be offered on site (i.e., via school-based centers) or off site (i.e., via school-linked centers).

This measure is a key indicator of collaboration between health and education sectors and aligns with an objective of Healthy People 2020 to institute school-based health programs (Inman et al., 2011). CDC has promoted the Whole School, Whole Community, Whole Child model to strengthen collaboration between schools and public health as a result of education, public health, and school health sectors calling for increased alignment with one another (CDC, 2014a). Because these sectors serve the same populations in the same settings, a collaborative approach results in more streamlined service delivery, more efficient use of resources, and shared responsibility for outcomes. In a Culture of Health, health will be prioritized across sectors, and people will be able to seek affordable professional care in environments that are accessible and integrated. SBHCs are emblematic of this goal.

CDC's Community Preventive Services Task Force recommends the implementation and maintenance of SBHCs in low-income communities, given the evidence for their effectiveness in improving educational and health outcomes. Improved educational outcomes include school performance, grade promotion, and high school completion, all of which contribute to lifelong health and well-being. Improved immediate health outcomes include greater delivery of vaccinations and other recommended preventive services, less asthma morbidity, fewer emergency department and hospital admissions, increased contraceptive use among females, improved rates of prenatal care, and higher birth weights (Lear, Eichner, and Koppelman, 1999; The Community Guide, 2015). 
Triennial census reports show that the number of SBHCs in the United States has consistently increased over the past decade (Schlitt et al., 2000; Juszczak et al., 2003; Juszczak, Schlitt, and Moore, 2007; Strozer, Juszczak, and Ammerman, 2010; Lofink et al., 2013). Legislation enacted between the 2007-2008 and 2010-2011 census data collection periods included the Children's Health Insurance Program Reauthorization Act of 2009, the Health Information Technology for Economic and Clinical Health Act, and the ACA. Each of these pieces of legislation resulted in grants to establish SBHCs or improve processes at SBHCs (Lofink et al., 2013). Moreover, research has shown that SBHCs continue to spread even in the absence of state funding or policies, likely due to support from local policies and third-party (private, university, or community-based) investment (Lear, Eichner, and Koppelman, 1999).

Index of employer health promotion and practices. Working-age adults spend a significant amount of their life working, so workplaces can have a significant impact on health (Leppo et al., 2013). Moreover, there is a strong business case for companies to invest in the health and wellbeing of their employees with respect to increased workforce well-being and productivity and reduced employer health care costs. A meta-analysis of workplace health promotion programs found that programs were generally successful in reducing absenteeism and increasing employees' productivity and work ability (Rongen et al., 2013). This measure reflects the presence of multiple policies and practices that encourage healthy behaviors (e.g., a tobacco-free workplace) and create healthy work environments (e.g., on-site locations for physical activity or stress management).

The CDC Community Preventive Services Task Force has recommended a number of evidence-based workplace health promotion strategies based on positive health impacts, including worksite signage to promote everyday physical activity and health risk assessments with feedback and access to a broad spectrum of health education programs to reduce behavioral health risks, such as tobacco use, excessive alcohol use, excessive dietary fat intake, and poor blood pressure control.

Current research indicates that workplace health promotion programs or employee health management programs can significantly improve multiple health outcomes. A systematic review conducted by $\mathrm{CDC}$ found that workplace health promotion and wellness programs increased awareness of health risks, detection of certain diseases with referral to medical professionals for high-risk employees, and the delivery of prevention and treatment programs tailored to the 
individual needs of the employee populations (CDC, 2007). The Annual Review of Public Health by Harris et al. (2014) documented that smaller employers with higher proportions of lowerincome and racial/ethnic minority employees and employees with lower levels of educational attainment are least likely to provide evidence-based worksite wellness programs and program components. These findings underscore the need for expanded workplace health promotion programs.

HERO has collected data on employer offerings of evidence-based workplace health promotion programs and policies consistent with a Culture of Health since 2009. The HERO scorecard covers the inclusion of health as part of a company's mission, goals, and values; policies related to employee health and well-being, including access to healthy food and physical activity opportunities; and the use of the physical environment to promote health. This index is new for HERO, so it has not yet tracked changes in these measures over time, but annual updates are expected to begin in 2015. Moreover, as the business case for employee health management programs continues to grow, employers will likely increase their support for these programs. In addition, future iterations of HERO's measures will assess business contributions to the health and well-being of the communities in which their employees reside.

\section{Resource Investments Across Sectors Measures}

We identified three measures to capture this driver.

Annual dollar amount of federal appropriation to selected community health initiatives. The federal government invests in numerous programs that support healthy behaviors at the community level (e.g., school nutrition programs, community transportation, walkability and green space development, food assistance for the poor, and Meals on Wheels for older adults living at home). Measuring how much of the federal budget is devoted to health initiatives and how much that changes annually is one way to gauge the federal government's investment in improving community health.

There is growing evidence that the Supplemental Nutrition Assistance Program (SNAP; formerly known as the Food Stamp Program) can dramatically improve the health of children. SNAP recipients have healthier foods (food energy, protein, vitamins) in their diets than do eligible nonparticipants (Devaney, Haines, and Moffitt, 1989), and SNAP can reduce the prevalence of child food insecurity, poor general health, obesity, and anemia (Kreider et al., 
2012). The U.S. Department of Agriculture (USDA) found that participation in the Special Supplemental Nutrition Program for Women, Infants, and Children (WIC) resulted in fewer pregnancies and premature births among recipients, fewer instances of low-birthweight babies, lower infant mortality, greater likelihood of prenatal care, and savings in health care costs within the first two months of birth (USDA Food and Nutrition Service, 2013b). Participation in WIC also has been shown to reduce racial disparities in the infant mortality rate between blacks and whites (Khanani et al., 2010). For seniors, programs such as Meals on Wheels have been shown to improve energy and nutrient intake (Roy and Payette, 2006).

Federal appropriations also can increase the physical activity levels of U.S. residents. There are promising findings on the relationship between overall access and/or quality of green space and physical activity (Coombes, Jones, and Hillsdon, 2010; Hillsdon et al., 2006; Maas et al., 2008), and there is strong evidence of green space's impact on the health of youth, elderly, and/or lower-income residents (Bell, Wilson, and Liu, 2008; Hume, Salmon, and Ball, 2005; Maas et al., 2006). Increased access to spaces for physical activity may be a way to reduce health inequalities (Mitchell and Popham, 2008). These findings are particularly important because funding for National Park Service projects depends on the fluctuating amounts that Congress appropriates annually to the Land and Water Conservation Fund.

Annual dollar amount of U.S. corporate giving to K-12 education, higher education, and community/economic development. With billions of dollars in donations of cash or services annually, corporations make substantial contributions to a Culture of Health through investments in the drivers of health and well-being. Tracking corporate giving for primary education, secondary education, and community and economic development — all of which are powerful determinants of health and well-being - will provide an index of corporate investments in initiatives that support population health and related economic outcomes.

Annual Committee Encouraging Corporate Philanthropy (CECP) investments in education and community development sectors were estimated at \$2.4 billion in 2014 (from a 2015 CECP custom analysis). Global research indicates that increasing education quality has positive impacts on income growth and mortality (Jamison, Jamison, and Hanushek, 2007). In the United States, numerous studies have made the link between such neighborhood factors as education and health outcomes (Pickett and Pearl, 2001). Moreover, the National Education Association (NEA) has conducted a number of studies that support increasing and distributing funding for education to 
enhance education quality (NEA, 2004a, 2004b, 2004c). Most of this research considers public education systems funded by public dollars, but corporate dollars could operate in a similar way.

Community development has been correlated with positive children's health outcomes (Komro et al., 2013) and population health broadly. Community development is particularly important to the improvement of well-being among low-income and other sociodemographically disadvantaged and high-risk populations (Erickson and Andrews, 2011). As government funding streams for education and community development fluctuate, philanthropic contributions often fill the gap. There is growing evidence to support the long-term community, regional, and national health and fiscal benefits from these investments (Erickson and Andrews, 2011).

Annual measure of children's exposure to TV ads for unhealthy and healthy foods/beverages. In 2006, the Institute of Medicine concluded that food marketing to children puts their current health and long-term health prospects at risk. Controlling such marketing will require a multipronged effort by government, media, food and beverage industries, restaurants, food stores, and the health sector to redirect the marketing resources of the food and beverage industries away from unhealthy foods. American children's diets are high in saturated fats, sugars, and sodium that add harmful excess non-nutritive calories to their diets and contribute to the current epidemic of childhood obesity, raising lifelong risks for Type 2 diabetes, heart disease, disability, and premature death. In 2011-2012, 22.8 percent and 34.2 percent of children ages 2-5 and 611 , respectively, were obese or overweight, with the highest prevalence among youth in racial/ethnic minority populations and disadvantaged low-income communities. Numerous studies have shown that overweight children are more likely to become overweight adults, especially if they have higher body mass indexes (BMIs) (Guo and Chumlea, 1999) or obese parents (Whitaker et al., 1997). Adolescent obesity is likely to continue into adulthood, although the persistence of higher BMIs into adulthood accounted for much of the association (Engeland, Tretli, and Bjørge, 2004).

The 2012 Federal Trade Commission report on food marketing to youth found that companies spent $\$ 1.79$ billion marketing food and beverages to youth, with just over $\$ 1$ billion directed at children ages 2-11-the largest proportion of which was spent on television advertising ( $\$ 632.7$ million, or 35.4 percent of marketing directed to that age group). Children see an average of 12-13 food-related ads per day, of which approximately eight or nine are for (non-restaurant) food and beverages. In 2006, the Council of Better Business Bureaus launched 
the Children's Food and Beverage Advertising Initiative (CFBAI), with food and beverage companies pledging to market healthy or "better-for-you" products to children. Despite the CFBAI, the vast majority of food and beverage product television advertisements seen by children on all programming ( 84 percent) and on children's programming ( 96 percent) continues to be for products that are high in saturated fat, sugar, or sodium. These ubiquitous exposures create and perpetuate unhealthy eating norms and preferences among U.S. children and their families and present a major impediment to building a Culture of Health around food and eating.

Progress in reducing unhealthy food marketing to children is occurring on many fronts, with measurable positive consequences. Sixteen of the largest U.S. consumer packaged goods companies reduced the number of (excess) calories (mainly from fats and added sugar) in their U.S. food and beverage sales by 6.4 trillion from 2005 to 2012 through changes in product formulation and marketing (National Collaborative on Childhood Obesity Research, 2014). These changes have both fueled and harvested growing consumer demand for healthier products, especially for children.

\section{Policies That Reflect and Encourage Collaboration Across Sectors Measures}

We identified three measures to capture this driver.

Percentage of full-time sworn law enforcement personnel who have served as community policing or community relations officers or were designated to engage regularly in community policing activities in the last year. Community-oriented policing refers to a national movement in policing in which the community is a partner with police in achieving public safety. A national community policing measure is important because it reflects a transition from traditional policing - primarily built on reactive strategies in response to crimes - to a model that fosters a positive relationship between the public and police and aims to restore legitimacy to law enforcement in the eyes of marginalized groups (Weisburd et al., 2012). Community-oriented policing affects crime rates or safety outcomes over time. Two recent Campbell Collaboration systematic reviews indicate that (1) restoring legitimacy of law enforcement in the eyes of communities does prevent crime; and (2) problem-oriented policing, which is often a part of community-oriented policing, directly prevents crime (Harris et al., 2014). Weisburd and Eck (2004) found in an early review of the literature that community meetings, foot patrols, and providing information about crime to the public had little effect on crime, but that door-to-door 
visits reduced crime and fear of crime, and general improvements in police-community interactions also reduced fear and concern about crime. Observing increases in communityoriented policing approaches through this measure would provide evidence of social change between law enforcement and the communities they serve, as an essential step on the pathway to healthier outcomes for many communities of color.

Annual percentage of states with climate action plans. Changes in the world's climate can seriously affect Americans' health through increasing the frequency and severity of weather events (e.g., heat waves), increasing severity of air pollution, and intensifying (through increased temperatures) the spread of infectious diseases. Climate effects are the most common factors influencing challenging weather events and are likely to increase the frequency, severity, and length of heat waves, which, among other things, can lead to stroke and dehydration. Climate effects will likely increase the frequency and intensity of extreme weather events, which can threaten food, water, communication, and health care supplies and services and contribute to injuries, death, and post-traumatic stress disorder (Ebi, Sussman, and Wilbanks, 2008; United States Global Change Research Program, 2009).

The process of developing a state climate action plan is an important initial step toward improving climate resilience and is an indicator that states have an interest, are mobilized, and have engaged multiple stakeholders to advise and agree on concrete actions. These stakeholders include the energy sector, transportation, car manufacturers, agriculture, and many other industries. To date, climate action plans have been published by 34 states and the District of Columbia.

Annual percentage of families with parents who are eligible for Family and Medical Leave Act coverage and can afford it, nationally and by state. The Family and Medical Leave Act (FMLA) entitles eligible employees of covered employers to take unpaid, job-protected leave for specified family and medical reasons. Complex health problems are influenced by many factors that cut across multiple sectors, such as socioeconomic policies, business involvement and support, education, and access to resources. A measure of FMLA access is important for understanding how the broader policy landscape can impact the health of Americans. It also signifies the role that business can play in advocating for policies that support employees.

Research indicates that parents' ability to participate in their children's health care is associated with better child developmental outcomes, including faster recovery periods. Yet 
access to family and sick leave through employer policies is unequal. Low-skill, low-wage, racial/ethnic minority, female, and immigrant workers are significantly more likely to have fewer employer-provided benefits, such as health insurance and less time for leave. Some of these populations are also more vulnerable to health conditions, which means that they may be the most in need of, but the least likely to access, worker benefits that can help ameliorate health issues.

New estimates show that 57 percent of all working fathers are eligible for FMLA leave, although only 42 percent earn enough to potentially take the unpaid leave. Black and Hispanic fathers face the dual challenge of eligibility and affordability, with only 25 percent of Hispanic fathers and 37 percent of black fathers being both eligible and able to afford FMLA leave (i.e., being able to maintain needed earning level if leave is taken) (Joshi et al., 2015). Across racial/ethnic groups, just over 50 percent of working parents are eligible for FMLA leave. Hispanic parents have the lowest eligibility rate (diversitydatakids.org, n.d.). States such as California, Rhode Island, and New Jersey have passed legislation to make family leave more affordable (diversitydatakids.org, n.d.) (e.g., provide some income to those who take unpaid leave).

The following table summarizes the measures described in the preceding sections. As noted earlier, these measures illustrate the three drivers for this action area (Table 6.3).

Table 6.3. Fostering Cross-Sector Collaboration to Improve Well-Being: Measures by Driver

\begin{tabular}{|l|l|l|}
\hline \multicolumn{1}{|c|}{ Driver } & \multicolumn{1}{|c|}{ Measure } & \multicolumn{1}{|c|}{$\begin{array}{c}\text { What It Means to the Action Area/ } \\
\text { Culture of Health Overall }\end{array}$} \\
\hline $\begin{array}{l}\text { Number and quality of } \\
\text { partnerships }\end{array}$ & $\begin{array}{l}\text { Percentage of local health } \\
\text { departments that collaborated with } \\
\text { community organizations (that do not } \\
\text { typically deal in health care) in at } \\
\text { least four public health program areas } \\
\text { in the last year }\end{array}$ & $\begin{array}{l}\text { Demonstrates that (1) public health is } \\
\text { influencing and influenced by different } \\
\text { sectors, and (2) public health prioritizes } \\
\text { reaching all community groups, even } \\
\text { those that are hard to reach }\end{array}$ \\
\hline $\begin{array}{l}\text { Number and quality of } \\
\text { partnerships }\end{array}$ & $\begin{array}{l}\text { Number of school-based health } \\
\text { centers that provide primary care }\end{array}$ & $\begin{array}{l}\text { Describes progress in integrating } \\
\text { education and health sectors; examines } \\
\text { opportunities for schools to impact the } \\
\text { health of community members beyond } \\
\text { schoolchildren }\end{array}$ \\
\hline $\begin{array}{l}\text { Number and quality of } \\
\text { partnerships }\end{array}$ & $\begin{array}{l}\text { Index of employer health promotion } \\
\text { and practices }\end{array}$ & $\begin{array}{l}\text { Provides insight regarding for-profit } \\
\text { sector commitment to health promotion }\end{array}$ \\
\hline
\end{tabular}




\begin{tabular}{|c|c|c|}
\hline Driver & Measure & $\begin{array}{l}\text { What It Means to the Action Area/ } \\
\text { Culture of Health Overall }\end{array}$ \\
\hline $\begin{array}{l}\text { Resource investment } \\
\text { across sectors }\end{array}$ & $\begin{array}{l}\text { Annual dollar amount of federal } \\
\text { appropriation to select health } \\
\text { initiatives }\end{array}$ & $\begin{array}{l}\text { Demonstrates the federal government's } \\
\text { commitment to improving the health of } \\
\text { U.S. residents }\end{array}$ \\
\hline $\begin{array}{l}\text { Resource investment } \\
\text { across sectors }\end{array}$ & $\begin{array}{l}\text { Annual dollar amount of U.S. } \\
\text { corporate giving to K-12 education, } \\
\text { higher education, and } \\
\text { community/economic development }\end{array}$ & $\begin{array}{l}\text { Provides scope or scale of corporate } \\
\text { investment in key sectors that may have } \\
\text { significant impact on the well-being } \\
\text { and future of communities }\end{array}$ \\
\hline $\begin{array}{l}\text { Resource investment } \\
\text { across sectors }\end{array}$ & $\begin{array}{l}\text { Annual measure of children's } \\
\text { exposure to TV ads for unhealthy and } \\
\text { healthy foods/beverages }\end{array}$ & $\begin{array}{l}\text { Describes the progress that media and } \\
\text { the food and beverage industry are } \\
\text { making with regard to improving } \\
\text { children's health }\end{array}$ \\
\hline $\begin{array}{l}\text { Policies that reflect and } \\
\text { encourage collaboration } \\
\text { across sectors }\end{array}$ & $\begin{array}{l}\text { Percentage of full-time sworn } \\
\text { personnel who have served as } \\
\text { community policing or community } \\
\text { relations officers or were designated } \\
\text { to engage regularly in community } \\
\text { policing activities in the last year }\end{array}$ & $\begin{array}{l}\text { Tracks the commitment of law } \\
\text { enforcement to achieving safe and } \\
\text { healthy communities, regardless of race } \\
\text { and income of residents }\end{array}$ \\
\hline $\begin{array}{l}\text { Policies that reflect and } \\
\text { encourage collaboration } \\
\text { across sectors }\end{array}$ & $\begin{array}{l}\text { Annual percentage of states with } \\
\text { climate action plans }\end{array}$ & $\begin{array}{l}\text { Reflects the progress of climate policy } \\
\text { in a state developed with input from } \\
\text { many sectors toward creating a } \\
\text { healthier physical environment in } \\
\text { which their residents can live }\end{array}$ \\
\hline $\begin{array}{l}\text { Policies that reflect and } \\
\text { encourage collaboration } \\
\text { across sectors }\end{array}$ & $\begin{array}{l}\text { Annual percentage of families with } \\
\text { parents who are eligible for FMLA } \\
\text { coverage and can afford it, nationally } \\
\text { and by state }\end{array}$ & $\begin{array}{l}\text { Describes a changing policy landscape } \\
\text { in which the health of workers and their } \\
\text { households is encouraged and valued }\end{array}$ \\
\hline
\end{tabular}

\section{Limitations}

The table above provides potential measures for evaluating progress in this action area, but gaps still exist in how we measure cross-sector collaboration.

\section{There are few available measures that assess the existence of relationships among non-}

health sectors. The Culture of Health measurement strategy will utilize measures of how sectors in which health is not the primary mission contribute to health and well-being. Those measures are at best novel and have not been rigorously tested. Further, there are few established measures regarding the quality of those collaborations. Quality might be measured by the strength of interactions among partners (e.g., trust, reciprocity, value), by the structure of the partnership (e.g., all participating as equals), or by the extent of the partnerships (e.g., resource-sharing, collaborations that require a significant time investment). But these quality measures do not 
systematically exist at the community level and are not distributed well enough for national aggregation.

Current efforts to track cross-sector investments are limited. This chapter recommends going beyond assessing the prevalence of partnerships to obtain critical information about how and how much cross-sectoral partners are investing in health. Such investment data are not consistently gathered in national databases or surveillance systems. The Culture of Health measurement strategy will need to motivate this rigorous tracking of philanthropic or nonprofit investments and the quality and nature of these investment data. Relatedly, future measurement for cross-sector collaborations needs to link fiscal and non-fiscal resource investment with actual population health outcomes. 


\section{Creating Healthier, More Equitable Communities}

This chapter provides an overview of the action area related to improved and equitable opportunity for healthy choices and environments. The goal of this action area is to advance health and well-being for all by improving the environments in which people live, learn, work, and play. This action area builds on and broadens RWJF's longstanding commitment to addressing the effects of the built, social, and policy environments on population health and health equity.

As with the other action areas, issues of health equity are woven into this area's fabric. We adopt Braveman's (2014) description of health equity as "striving for the highest possible standard of health for all people and giving special attention to the needs of those at greatest risk of poor health based on social conditions." Improving equitable opportunities and conditions for healthy choices and behaviors includes efforts to address disparities in access to resources that enable, foster, and support individual and population health and well-being. These resources reside in the built and physical environment, the social and economic environment, and the policies and governance that shape local contexts.

Substantial research shows persistent, significant, and inequitable differences in the conditions that create health and the opportunities to be healthy (Acevedo-Garcia and Lochner, 2003; Acevedo-Garcia et al., 2003; Williams and Collins, 1995). During the past two decades, a compelling body of evidence has helped to quantify the role of social factors, apart from medical care, in shaping health across a wide range of health indicators and across the life span. Just how social factors are related to health, however, is complicated, and there are varying degrees of evidence as to the strength and directionality. Yet, strong links between poverty and health have been observed for centuries (Foege, 2010; Hamlin, 1998; Rosen, 1993). In this chapter, we present the vision and action area definition, its primary drivers, and the measures chosen to catalyze and assess progress in this action area.

\section{Envisioning and Defining This Action Area}

The vision for this action area is to ensure that the physical, social, and economic environments in which people live, work, and play make it easier for them to make healthy 
choices, particularly minority racial/ethnic populations and those in low-income areas, where resources and healthy options are often limited. In fact, there is mounting evidence that residential ZIP codes can predict lifelong health and mortality.

Recent work has reinforced the importance of addressing structural drivers of risk (Braveman, 2014). Some work has identified racial residential segregation in the United States as perhaps the most fundamental determinant of differences in access to social and economic resources, from schools to economic retail to services and activities that promote health behaviors (Acevedo-Garcia and Lochner, 2003; Acevedo-Garcia et al., 2003; Williams and Collins, 1995). One measure of the health and well-being of children and the overall health of a community is infant mortality_infant death before age one. It reflects the status of maternal health that was likely influenced prior to conception, the accessibility and quality of primary health care, and the availability of supportive services in the community that extends throughout the life course into late adulthood. Geographic variation in infant mortality has been recognized and studied for decades (Kleinman, Feldman, and Mugge, 1976): evidence illustrates the clear racial disparities. Black infants in the United States are nearly three times more likely to die before their first birthday than are non-Hispanic white infants (Lu and Halfon, 2003; Mathews and MacDorman, 2010). While specific causes of infant death are likely complex, women's exposure to stressful life events prior to conception is associated with very low birth weight, and this association has been shown to be strongest in disadvantaged neighborhoods (Witt et al., 2015).

Social and physical aspects of neighborhoods, including streets where residents feel safe, maintained sidewalks for walking, well-designed and well-run programs at parks, and quality after-school programs, can encourage residents to engage in everyday physical activity and "active transport," such as walking or cycling to work. Research has found associations between greater distance to parks and lower levels of engagement in physical activity and sports activities (Boone-Heinonen et al., 2010), as well as mental health outcomes (Sturm and Cohen, 2014). Communities that provide easily accessible and affordable nutritious food make it easier to adopt and maintain healthy eating habits. Similarly, people are more likely to receive recommended medical care if facilities are accessible, either because they are located nearby or because safe, convenient transportation is available. 
Given the importance of such large-scale investments and resources, ensuring the opportunity for healthy choices for all in our diverse nation requires efforts at the community and organizational levels that recognize how the health of individuals is influenced by "upstream" factors, which represent many non-health sectors-including (but not limited to) housing, transportation, employment, and education.

\section{Drivers in the Action Area of Creating Healthier, More Equitable Communities}

Based on the literature review and stakeholder analysis, we identified three primary drivers for creating healthier, more equitable communities: (1) the built environment and physical living conditions, (2) the social and economic environment, and (3) policy and governance. We finalized these three as primary drivers because the literature review and stakeholder input noted that more investment and progress needed to be made in the physical, social, and economic aspects of community, and that decisionmaking structures that could effectively catalyze and maintain progress in each aspect were often missing. The first two drivers focus on the extent to which health-promoting physical, social, and economic environments are in place and equitably distributed. The third driver spotlights policies aimed at creating healthy environments, with an emphasis on collaboration between residents and large government and corporate institutions.

\section{Built Environment and Physical Conditions}

There is strong and growing evidence that the built environment - the physical space in which we live, learn, work, and play - affects a community's health and well-being by shaping its residents' abilities to make healthy choices and engage in healthy behaviors (Jackson et al., 2000). Many evidence-based strategies exist for increasing population-level physical activity, from urban design and land-use policies that address proximity of residential areas to stores, jobs, schools, and recreation areas to measures that improve street lighting, landscaping, and traffic. For example, sidewalks that are in good condition, marked crosswalks, and bicycle lanes are all features of the physical environment that can encourage active transportation (National Complete Streets Coalition, 2015).

Increased access to recreational facilities may promote exercise (Ding et al., 2011), which is associated with less risk of chronic disease and better mental health (Kruk, 2007; Peluso and Andrade, 2005). Similarly, increased access to healthy food sources may make a healthy diet 
more attainable (Bell et al., 2013). Just as importantly, food retail investments in areas that need access to healthy options may improve residents' satisfaction with their neighborhood as a place to live and promote additional economic investment at a community and neighborhood level (Dubowitz et al., 2015). At the same time, perceived and actual safety issues, such as blight, lack of street lighting, vandalism, and poorly maintained landscaping, prevent residents from taking advantage of opportunities within their neighborhoods (National Complete Streets Coalition, 2015).

In addition to promoting healthy choices, research has suggested that the physical environment affects social interaction and community connectivity (Carver, Timperio, and Crawford, 2008; Chang and Christakis, 2005; Kuo, 2001; Kuo and Sullivan, 2001), which can impact individual and population-level health and well-being (Lund, 2002, 2003; Talen, 1999). One review of 32 quantitative studies of the relationship between the neighborhood physical environment and early child health and development found "strong empirical evidence that neighborhoods which are safe from traffic and which have green spaces (i.e., nature, public open space, parks, playgrounds) are associated with behaviors (i.e., outdoor play and physical activity) that facilitate early child health and development," including social competence, social and emotional functioning, and pro-social behavior (Christian et al., 2015). In the same vein, there is growing evidence that the design, condition, and maintenance of the buildings and facilities where we live and work influence comfort, productivity, and well-being (Fisk, 2002).

Efforts to improve physical living conditions can include programming within local parks and green spaces, improving perceptions of neighborhood safety, and local commitment to address or adapt to climate change. For example, municipalities or communities that have plans to address changes in climate, such as from rising sea levels or changes in precipitation (which can include adaptations, such as flood plain management, or use of green or environmentally aware approaches to construction), are often better equipped to respond to evolving demographic and economic conditions and thus are better able to withstand a range of stressors (Uscher-Pines, Chandra, and Acosta, 2013).

The factors shown in Table 7.1 summarize how the built environment and physical conditions at the individual, organization/community, and policy environment levels can be shaped. 
Table 7.1. Factors Influencing the Built Environment and Physical Conditions

\begin{tabular}{|l|l|}
\hline Level & Factors \\
\hline Individual & - $\begin{array}{l}\text { Preference for healthy options or location } \\
\text { - Attitudes toward the physical environment (e.g., pride in place, use of } \\
\text { services) }\end{array}$ \\
\hline Organization/community & - $\begin{array}{l}\text { Investment in community design and planning } \\
\text { - Advocacy for policies that support healthier options }\end{array}$ \\
\hline $\begin{array}{l}\text { Decision environment/ } \\
\text { policies }\end{array}$ & - Availability of options for employees or constituents \\
\hline
\end{tabular}

\section{Social and Economic Environment}

One's social environment includes social relationships and cultural milieus within which groups of people function and interact, as well as the residential demographics of where people live. How people interact with these spaces and how they are managed can determine their health behaviors (alcohol consumption, tobacco use, physical activity, diet), frequency of illness, receipt of medical treatment, and even their odds of premature death (Sleddens et al., 2015). Economic and educational opportunities, affordable housing, opportunities for cultural and political expression, and other positive social determinants can all influence one's health and well-being. Other components of the social environment include (but are not limited to) labor markets; wealth; social, human, and health services; and religious institutions and practices.

Strong links between poverty and health have been observed and documented over centuries (Braveman and Gruskin, 2003; Galea and Vlahov, 2005). In the United States, especially, neighborhoods can be segregated by race/ethnicity or socioeconomic status, which influences many social features, such as social trust and social cohesion. In the United States, racial and socioeconomic segregation in residential areas has been linked to outcomes including low birth weight (O’Campo et al., 1997), illness-related disability (Shouls, Congdon, and Curtis, 1996), and overall mortality (Bird and Bauman, 1998; Hertz, Hebert, and Landon, 1994; LeClere, Rogers, and Peters, 1997; Polednak, 1996).

Much research has focused on two aspects of the economic environment: job opportunities and land-use mix (e.g., the proximity of residential and commercial districts). With respect to job opportunities, we know that unstable or short-term employment is associated with poor health outcomes (Artazcoz et al., 2005; Kim et al., 2006; Kivimäki et al., 2003). In terms of land-use 
mix, residents in neighborhoods and communities that lack diverse land use tend to have higher BMIs (Frank, Andresen, and Schmid, 2004), use less active transit modes, and are exposed to higher levels of air pollutants (Frank et al., 2006). One recent review found that mixed land use, as well as several other aspects of community development, was associated with increased physical activity. Specifically, mixed land use, diverse housing types, housing density, compact development patterns, and open space were generally associated with higher levels of physical activity (Durand et al., 2011).

Economic vitality also is essential to a Culture of Health and can include such indicators as involuntary unemployment or productivity. A number of groups are vulnerable based on life circumstances (e.g., a lack of economic, cultural, or social resources), and these experiences can impede well-being (Cutter, Mitchell, and Scott, 2000; Mechanic and Tanner, 2007; Norris et al., 2008). When these populations are not reaping the benefits of economic resources and productivity, it becomes more difficult for the entire community to develop and maintain resilience in the face of any stress (Norris et al., 2008). For example, communities that do not have strong economic structures have difficulty supporting any type of recovery; bringing jobs back to the community; or returning to a normally functioning, viable workforce. To build and maintain health, communities must engage in economic development and reduce social and economic inequities. According to Pfefferbaum et al. (2005), resilience and, ultimately, community health and well-being depend on ongoing investments in physical resources, such as schools, health facilities, job training programs, and neighborhood development.

Table 7.2 lists factors that influence the social and economic environment.

Table 7.2. Factors Influencing the Social and Economic Environment

\begin{tabular}{|c|c|}
\hline Level & Factors \\
\hline Individual & $\begin{array}{l}\text { - Participation in social and health-promoting activities, employment training } \\
\text { programs, or economic development programs }\end{array}$ \\
\hline Organization/community & $\begin{array}{l}\text { - Supportive wellness policies } \\
\text { - } \quad \text { Availability of social options that promote health/avoid health risk }\end{array}$ \\
\hline $\begin{array}{l}\text { Decision environment/ } \\
\text { policies }\end{array}$ & $\begin{array}{l}\text { - Policies that reduce health risk behaviors (e.g., anti-smoking legislation) } \\
\text { - Job training, other employment policies } \\
\text { - } \quad \text { Earned income tax credit policies }\end{array}$ \\
\hline
\end{tabular}




\section{Policy and Governance}

The policy and governance driver emphasizes the influence on populations from both government and private entities. Policies can range from worksite promotion of healthy living (e.g., parental leave policies, on-site healthy food options) to large efforts to reduce racial and socioeconomic residential segregation, such as deconcentrating housing choice vouchers from the central city to the suburbs or allowing housing choice voucher holders to move to resourceabundant (instead of resource-challenged) neighborhoods with high-quality services and schools (Anderson et al., 2002). Smoke-free policies and indoor air acts, cigarette taxes, healthy school environment policies, and even incentives from programs, such as WIC, to purchase fruits and vegetables are examples of higher-level actions that can shape environments and behaviors.

In a systematic review and meta-analysis of North American and European studies, smokefree legislations were linked to reductions in preterm birth and hospital attendances for asthma (Been et al., 2014). Another U.S. study investigating associations of state-specific smoke-free laws found significantly lower rates of hospital admissions or deaths for coronary events, other heart disease risk, cerebrovascular accidents, and respiratory disease. Additionally, more comprehensive laws were further associated with larger decreases in risk (Tan and Glantz, 2012).

One large-scale example is complete streets policies focused on improving transportation infrastructure for all users, including pedestrians, drivers, and riders of public transit. Such policies typically focus on bike lanes, traffic-calming measures, and infrastructure for pedestrians to promote safe access for all (Moreland-Russell et al., 2013).

For many of these efforts (including complete streets policies), enactment and implementation tend to rely on access to resources for implementation (including monitoring or evaluation), a civically engaged community, or champions and advocates of specific policies (Brain, 2005; Coaffee and Healey, 2003; Docherty, Goodlad, and Paddison, 2001; Podobnik, 2002; Taylor, 2000). For example, Smart Growth America identifies the day-to-day decisions that a transportation agency and community leaders make in funding, planning, design, maintenance, and operations as some of the most important determinants of whether complete streets policies are implemented. These actions need to align with the goals of the adopted policy. The National Complete Streets Coalition has identified five activities required to reorient a transportation agency's work to fully and consistently consider the safety of all users: (1) assessing all current procedures and activities and planning for the implementation of complete 
Streets; (2) updating documents, plans, and processes used in transportation decisionmaking, from scoping to funding, and creating new ones if necessary; (3) updating or adopting new design guidance and standards to reflect current best practices in providing multimodal mobility; (4) providing ongoing support to transportation professionals, other relevant agency staff, community leaders, and the public so they understand the complete streets approach, the new processes and partnerships it requires, and the potential new outcomes from the transportation system; and (5) creating or modifying existing metrics to measure success in accommodating all users on the project and network levels.

Many health-supporting policies and investments involve sectors not traditionally viewed as health sectors, such as transportation, education, and urban design, so it is important to examine whether communities have governance processes in place to provide venues for discussion of health and equitable access to health and health-related services and community conditions. These factors overlap with drivers in other action areas, particularly shared values (civic engagement) and multi-sector collaboration (McQueen et al., 2012).

Factors that influence policy and governance on the individual, organization/community, and decision environment levels are shown in Table 7.3.

Table 7.3. Factors Influencing Policy and Governance

\begin{tabular}{|c|c|}
\hline Level & Factors \\
\hline Individual & $\begin{array}{l}\text { Endorsement of health-promoting policies, investment choices for community } \\
\text { health and well-being }\end{array}$ \\
\hline Organization/community & - Organizational participation in health-promoting policy development \\
\hline $\begin{array}{l}\text { Decision environment/ } \\
\text { policies }\end{array}$ & $\begin{array}{l}\text { - Inclusive governance policies for citizen voices about health } \\
\text { - } \quad \text { Creation of community councils to review health policy trade-offs }\end{array}$ \\
\hline
\end{tabular}

\section{Measures}

The following measures were identified as capturing key aspects of each driver. We describe each measure in further detail below and then summarize them in Table 7.4. Note that more detail on how each measure is operationalized is provided on the Culture of Health website (see www.cultureofhealth.org). 


\section{Built Environment and Physical Conditions Measures}

We identified three measures to capture this driver.

Percentage of families spending excessive levels of monthly income on housing costs for either rent or mortgage. Housing is important to health in many ways, from exposure to toxins to the safety of where people live. When households struggle with housing costs, they are less able to buy food, use transportation to get to work, and afford regular health care (Cohen, 2011). The stress of not meeting housing payments or having housing instability can also have negative mental health consequences (Krieger and Higgins, 2002). A Culture of Health values the creation of conditions to help people have safe and secure homes and spend their resources on activities that promote well-being rather than simply keeping a roof over their heads.

Affordable housing can affect health directly and indirectly. Physical conditions of homes, social and physical conditions in neighborhoods surrounding homes, and the costs of housing shape both home and neighborhood conditions and the ability of families to make healthy choices. A shortage of affordable housing limits families' and individuals' choices about where they live, often relegating lower-income families to substandard housing in neighborhoods that may have higher rates of poverty, crime, and density and fewer parks, bike paths, recreation centers, and other activities. The financial burden of unaffordable housing can prevent families, particularly low-income households, from meeting other basic needs, including nutrition and health care (Dunn, 2000; Pollack, Griffin, and Lynch, 2010). Lower-income populations typically spend a higher percentage of their income on housing than their wealthier peers, making housing affordability fundamentally an issue of equity.

The data for this driver are related to income status and economic disparity. The U.S. Department of Housing and Urban Development estimates that 12 million renters and homeowners spend more than 50 percent of their annual income on housing. In addition, it reports that "a family with one full-time worker earning the minimum wage cannot afford the local fair-market rent for a two-bedroom apartment anywhere in the United States" (U.S. Department of Housing and Urban Development, n.d.[a]).

The Moving to Opportunity for Fair Housing Demonstration Project, a randomized controlled experiment in five cities, was designed to test long-term effects on well-being and health associated with moving from high-poverty areas to private-market housing in lowerpoverty neighborhoods through the use of vouchers. While early findings suggested favorable 
outcomes for families, the longer-term effects varied by the age and sex of the participants (U.S. Department of Housing and Urban Development, n.d.[b]). Compared with families that had similar resources and characteristics but did not receive the vouchers, adults who received vouchers and were able to obtain housing in low-poverty areas experienced significant improvements in neighborhood satisfaction and safety, prevalence of psychological distress and depression, and obesity. Among teenagers, girls experienced improved mental health and reported fewer risky behaviors; boys, however, actually experienced adverse outcomes, including more delinquency and risky behaviors (Isaacs, 2012), which some have speculated could be due to the stresses of moving - and specifically of moving to areas where most peers were doing better.

Housing policies that have shown positive impacts on health include subsidies to low-income families that enable them to rent in the private sector and public housing design that promotes health. Identifying, monitoring, and evaluating housing policies and their implementation throughout the United States would allow documenting and measuring the growth and effect of such policies.

Percentage of U.S. population with limited access to healthy foods. Access to healthy food is important to community health, but in some communities it is difficult to obtain fresh food, and fresh produce in particular. As a result, some communities must rely on limited or poor-quality food, which impedes nutrition and ultimately hampers the development of positive health outcomes.

Although the research is mixed as to whether living closer to a supermarket is associated with a healthier diet (An et al., 2013; Lee, 2012), limited access to healthy food options has important health and economic implications. We know that in-store marketing, price, and the variety and quality of healthy foods available are also important factors (Andreyeva, Long, and Brownell, 2010; Robert Wood Johnson Foundation, 2011). Data on food variety and quality are limited, so the proxy for access to healthy food is limited to the best available data based on 2010 estimates compiled by the USDA (Ver Ploeg, 2012) (the USDA definition of low access is living more than one mile from a supermarket, supercenter, or large grocery store in an urban area, or living more than ten miles from a supermarket in a rural area; USDA, 2013).

The economic impact of food access in low-income geographic areas, often represented by census tracts or small, relatively stable geographic units, frequently used for the presentation of 
statistical data, is also valuable: Supermarkets provide opportunities for employment, bring in revenue to the neighborhood (the Reinvestment Fund, 2013), and may incentivize other economic infusions. In addition, studies have shown that the presence of supermarkets increases property values and spurs community development (Econsult Corporation, 2013).

Although the number of census tracts with low access to healthy foods increased from 2006 to 2010 , most of this is due to an increase in low-income census tracts. The number of lowaccess tracts grew from 27,776 to 28,328 , while low-income tracts grew by a larger margin, from 26,099 to 29,134 . Data demonstrate that counties with higher proportions of blacks and/or Hispanics have higher shares of people living in low-access, low-income census tracts than counties with higher proportions of whites (USDA, 2013). Expansion and renewal of the Healthy Food Financing Initiative, which supports projects that increase access to healthy, affordable food in communities that currently lack these options, offered new policy supports for farmers markets and expanded WIC and SNAP benefits.

Percentage of middle and high school students reporting feeling safe in their communities and schools. Feeling safe influences trust, the motivation to go to school, and the motivation to be active outside. In schools, perceived safety affects truancy, attendance, and performance for both in-school and extracurricular activities (Henrich et al., 2004). In communities, perceived safety affects outdoor physical activity and recreation. In individuals, negative safety perceptions can increase acute or chronic stress and/or anxiety (Burdick-Will, 2013). Overall, feeling unsafe in one's community can create numerous barriers to healthy living. A study of 306 residents in New Jersey found that improving perceived safety requires improving neighborhood schools, controlling unwanted land use, improving neighborhood conditions, and improving relations between the community and such authorities as local officials, investors, or police (Greenberg, 1999).

\section{Social and Economic Environment}

We identified three measures to capture this driver.

Evenness with which racial/ethnic groups are distributed across metropolitan areas to measure dissimilarity and diversity exposure. Racial residential segregation divides people into separate neighborhoods that often do not have the same economic or educational opportunities. Evenness - or the differential distribution of a population across neighborhoods in a given 
metropolitan region - reflects this spatial separation in housing markets (Lofink et al., 2013). Physical separation of communities by race can impact access to quality education, services, and socioeconomic mobility across the life course and can result in concentrated wealth and poverty. A recent review showed that residential segregation is associated with black mortality, black infant mortality, black homicide rates, teenage childbearing, tuberculosis, cardiovascular disease, availability of healthy food, and exposure to toxic air pollutants (Kramer and Hogue, 2009).

National trends show a slow but steady decline in black/white segregation from 1980 to 2010 (a 19-percent decline). The decline has been larger in areas with small black populations, such as Portland, Ore., but is occurring steadily in areas with moderate and large black populations (Iceland and Sharp, 2013).

While there is limited strong evidence on how to modify residential segregation, certain types of voucher programs have had modest effects: rental voucher programs, the Moving to Opportunity voucher and counseling program, and a program in Chicago that provided vouchers that enabled residents in highly segregated neighborhoods to live in racially integrated neighborhoods (Kramer and Hogue, 2009).

Number of states in which 60 percent or more of 3- and 4-year-olds are enrolled in preschool. There is a positive relationship between access to early childhood educational opportunities and child development, and there are both direct and indirect pathways between early childhood education and health. For example, children who attend preschool are 9 percent more likely to be "school-ready" than children who do not attend (Isaacs, 2012), and school readiness at an early age is linked to better success in grade school and lower high school dropout rates (Duncan et al., 2007). Especially for children in poverty, social and cognitive support is important to health and developmental outcomes. Early education can also improve access to health services, including vaccinations, health and dental screenings, and appointments with social workers and psychologists (Friedman-Krauss and Barnett, 2013).

Early childhood education is a core and lifelong equity issue. The Early Childhood Longitudinal Study found that just 48 percent of poor children were ready for school at age 5 , compared with 75 percent of children from families with moderate and high incomes (Isaacs, 2012). With such longitudinal studies, there is now evidence of the lifelong benefits of early childhood programming and learning, such as better health, higher earnings, lower likelihood of being on public assistance, and lower likelihood of committing a crime (Barnett, 2011). Investing 
in our youngest children is one of the three core Culture of Health strategies proposed by RWJF's Commission for a Healthier America. The others are creating stronger quality standards for early childhood education and guaranteeing access to certified programs for all low-income children under 5.

A 2004 National Institute for Early Education Research (NIEER) analysis found that while 78 percent of 3- and 4-year-olds from families with incomes over $\$ 100,000$ attended preschool, less than half of children from families with incomes below \$50,000 did so. Among Hispanics, just 23 percent of 3-year-olds and 50 percent of 4-year-olds attend preschool, according to national enrollment data. Black children attend preschool in roughly the same percentages as their white peers (about 45 percent of 3-year-olds and 70 percent of 4-year-olds) (NIEER, 2004).

Number of library outlets per 100,000 people. Public libraries serve as information hubs and free, safe spaces for social interaction, which positions them to support collective action to address social problems. Libraries also provide information important for voting and offer business resources, especially for small local businesses. In addition, public libraries promote community and inclusion through language and other resources for immigrants; non-English speakers; lesbian, gay, bisexual, transgender, and queer communities; and those with disabilities (Edwards, Rauseo, and Unger, 2013). A Pew survey found that "ninety-six percent of [respondents] agreed that public libraries are important because they provide tech resources and access to materials, and the same number found public libraries valuable because they promote literacy and a love of reading" (Zickuhr et al., 2013). In addition, the ACA calls for a partnership with public libraries to help residents understand their health care options and to register online for health insurance, thus playing a central role in health literacy (American Library Association, 2014).

As recent New York Times articles have noted (Kimmelman, 2013), libraries are emerging as critical to the revitalization of struggling or depressed communities (e.g., Chicago, Ill.; Hudson, Ohio; and Putney, Vt., where libraries transformed into the hubs of neighborhoods). They also often serve as a refuge during a disaster, which was apparent during the 1995 heat wave in Chicago, when some poor neighborhoods fared better than others because they had libraries or other cool places to go to escape the heat (Seville, 2009). Thus, libraries are important partners in sustainability and serve as a resource and a safe space for the aging population in communities. Lastly, they also often promote the arts and child development through programming. 


\section{Policy and Governance}

We identified two measures to capture this driver.

Number of jurisdictions with complete streets policies. Complete streets policies reflect the local policy environment around integrating multimodal transportation and improving pedestrian walkability and safety, and they reflect the integration of community and population health into transportation. Complete streets policies support roads that are safe, comfortable, and convenient for people of all ages and abilities to walk, bike, ride public transportation, and drive on. Policies aim to transform streets from a public health barrier to an asset by addressing roadway injuries and promoting walking and biking. There are also economic benefits. For example, the transformation of Eighth Street SE in Washington, D.C., sparked the opening of 32 new businesses and an additional $\$ 80,000$ in sales taxes annually. Complete streets policies can help curb transportation-related air pollution — responsible for almost one-third of all greenhouse gas emissions - by reducing traffic congestion and the number of trips made by car (National Complete Streets Coalition, 2015).

In 2013, more than 90 jurisdictions adopted a complete streets policy. The policies address fundamental issues of equity, as people of color, older adults, children, and low-income people suffer disproportionately from poor street design because of an increased likelihood of living in neighborhoods that do not attain air quality standards and of not getting enough physical activity. For those with disabilities, a prevalence of "incomplete" streets increases the likelihood of being isolated and dependent (National Complete Streets Coalition, 2015).

Complete streets policies transform streets from a public health barrier to an asset, enabling a greater return on the investment of public dollars. Such policies help address roadway injuries (2.5 million people each year [CDC, 2015b]), driving fatalities (motor vehicle crashes are the leading cause of death for U.S. teens, accounting for more than one-third of fatalities [CDC 2015a]), motor vehicle accident expenses (about \$164 billion annually in property damage and injuries), and obesity. Complete streets policies can help curb transportation-related air pollution, which is a trigger for asthma (4,000 die each year from asthma-related causes) and a huge cost (an estimated $\$ 40$ billion to $\$ 64$ billion annually in associated health issues) (U.S. Department of Transportation, 2009). Complete streets policies can also improve access to important facilities (almost one-third of the U.S. population is "transportation disadvantaged"), thus boosting the economy, and they can increase fuel efficiency by reducing traffic congestion (fuel lost during 
traffic jams cost $\$ 78$ billion in 2007) and reducing trips by car (28 percent of all trips are one mile or less, and 72 percent of the shortest trips are now made by automobile) (Smart Growth America, 2016).

Percentage of population covered by comprehensive smoke-free indoor air laws by state. Maintaining air quality is a key aspect of creating healthy environments. A 2006 study by the U.S. Surgeon General found that secondhand smoke increased the risk of disease, including lung cancer, in nonsmokers. Further, the children of parents who smoke have a higher likelihood of developing respiratory infections (U.S. Department of Health and Human Services, 2006). Since then, 38 states have passed smoke-free air laws that typically target workplaces, restaurants, and bars. These laws have not only been successful in separating nonsmokers and smokers, but they have also decreased the overall number of people smoking in workplaces. The laws have been linked to improved quality of life and increased profits for restaurants and other venues. Smokefree laws have also been linked to lower numbers of asthma incidents at the county level (Kabir, Arora, and Alpert, 2011).

Table 7.4 displays the measures selected for each of the three drivers in this action area.

Table 7.4. Creating Healthier, More Equitable Communities: Measures by Driver

\begin{tabular}{|l|l|l|}
\hline \multicolumn{1}{|c|}{ Driver } & \multicolumn{1}{|c|}{ Measure } & \multicolumn{1}{c|}{$\begin{array}{c}\text { What It Means to the Action Area/ } \\
\text { Culture of Health Overall }\end{array}$} \\
\hline $\begin{array}{l}\text { Built environment } \\
\text { and physical } \\
\text { conditions }\end{array}$ & $\begin{array}{l}\text { Housing affordability } \\
\text { (percentage of families } \\
\text { spending 50 percent or more } \\
\text { of monthly income on rent or } \\
\text { mortgage) }\end{array}$ & $\begin{array}{l}\text { Offers an indicator of housing challenges because } \\
\text { affordability can shape health, from individual } \\
\text { exposure to toxins to the safety of the } \\
\text { neighborhood/community. }\end{array}$ \\
\hline $\begin{array}{l}\text { Built environment } \\
\text { and physical } \\
\text { conditions }\end{array}$ & $\begin{array}{l}\text { Access to healthy foods } \\
\text { (percentage of U.S. population } \\
\text { with limited access to healthy } \\
\text { foods) }\end{array}$ & $\begin{array}{l}\text { Offers an indicator of community health as well as } \\
\text { equity. Low-income and racial/ethnic minority } \\
\text { populations disproportionately live in food deserts } \\
\text { with very limited access to full-service grocery } \\
\text { stores and an abundance of convenience stores that } \\
\text { do not stock healthy foods. }\end{array}$ \\
\hline $\begin{array}{l}\text { Built environment } \\
\text { and physical } \\
\text { conditions }\end{array}$ & $\begin{array}{l}\text { Youth safety (percentage of } \\
\text { middle and high school } \\
\text { students reporting feeling safe } \\
\text { in their communities and } \\
\text { schools) }\end{array}$ & $\begin{array}{l}\text { Provides an indicator of perceived safety. Feeling } \\
\text { unsafe influences trust in others, motivation to go } \\
\text { to school, and motivation to be active outside. } \\
\text { Perceived safety in schools also affects truancy, } \\
\text { attendance, and performance. }\end{array}$ \\
\hline $\begin{array}{l}\text { Social and } \\
\text { economic } \\
\text { environment }\end{array}$ & $\begin{array}{l}\text { Public libraries (number of } \\
\text { library outlets per 100,000 } \\
\text { people, nationally and by } \\
\text { state) }\end{array}$ & $\begin{array}{l}\text { Provides an indicator of a well-being asset, as } \\
\text { libraries represent investment in communities, } \\
\text { facilitate access to services, and are a linchpin to } \\
\text { literacy-a critical factor in health. }\end{array}$ \\
\hline
\end{tabular}




\begin{tabular}{|l|l|l|}
\hline \multicolumn{1}{|c|}{ Driver } & \multicolumn{1}{|c|}{ Measure } & \multicolumn{1}{c|}{$\begin{array}{c}\text { What It Means to the Action Area/ } \\
\text { Culture of Health Overall }\end{array}$} \\
\hline $\begin{array}{l}\text { Social and } \\
\text { economic } \\
\text { environment }\end{array}$ & $\begin{array}{l}\text { Early childhood education } \\
\text { (number of states in which } 60 \\
\text { percent or more of 3- and 4- } \\
\text { year-olds are enrolled in } \\
\text { preschool) }\end{array}$ & $\begin{array}{l}\text { Offers an indicator of lifelong healthy cognitive } \\
\text { and social emotional development through school } \\
\text { readiness and early learning gains that increase } \\
\text { high school graduation, reduce crime, and reduce } \\
\text { teen pregnancy rates. }\end{array}$ \\
\hline $\begin{array}{l}\text { Social and } \\
\text { economic } \\
\text { environment }\end{array}$ & $\begin{array}{l}\text { Residential segregation } \\
\text { (evenness with which } \\
\text { racial/ethnic groups are } \\
\text { distributed across } \\
\text { communities, both diversity } \\
\text { exposure and index of } \\
\text { dissimilarity) }\end{array}$ & $\begin{array}{l}\text { Offers a clear marker of equity, as physical } \\
\text { separation of communities by race affects access } \\
\text { to quality education, services, and socioeconomic } \\
\text { mobility across the life course and can result in } \\
\text { concentrated wealth and poverty. This can } \\
\text { ultimately influence access to health-promoting } \\
\text { behaviors and health care services. }\end{array}$ \\
\hline $\begin{array}{l}\text { Policy and } \\
\text { governance }\end{array}$ & $\begin{array}{l}\text { Complete streets policies } \\
\text { (number of jurisdictions with } \\
\text { these policies in place) }\end{array}$ & $\begin{array}{l}\text { Demonstrates community advocacy for and } \\
\text { investment in creating healthy physical } \\
\text { environments. For example, multimodal } \\
\text { transportation improves pedestrian walkability and } \\
\text { safety and reflects the integration of community } \\
\text { and population health into transportation. }\end{array}$ \\
\hline $\begin{array}{l}\text { Policy and } \\
\text { governance }\end{array}$ & $\begin{array}{l}\text { Air quality (percentage of } \\
\text { population covered by } \\
\text { comprehensive smoke-free } \\
\text { indoor air laws, by state) }\end{array}$ & $\begin{array}{l}\text { Shows community action on behalf of health and } \\
\text { integration of business policies. Smoke-free laws } \\
\text { significantly reduce tobacco-related asthma } \\
\text { incidence, cardiovascular disease, and sudden } \\
\text { cardiac events and deaths; deter smoking initiation } \\
\text { and promote cessation; and have been found to } \\
\text { boost business and revenues for bars and } \\
\text { restaurants. }\end{array}$ \\
\hline
\end{tabular}

\section{Limitations}

Each driver and its associated measures represent important areas to track over time, but there are limitations.

\section{Some of the evidence on the association between the built environment and health is}

tenuous. As one example, the data are mixed as to whether increased access to healthy food choices changes diet. More work to capture and describe the retail food environment (e.g., whether it is possible to capture variability from supermarket to supermarket or the marketing and promotion of less healthy versus healthy choices) and understanding how individuals engage and are affected by the services and stimuli around them could help uncover the relationships between built environment and health. As another example, retail food indexes do not account for variation in quality from store to store, by neighborhood, and by region, and we do not know whether and how residents engage with their closest stores. To track progress in this action area, the measurement strategy should determine ways to accurately capture built environment 
variability by region (e.g., rural/urban, other) and to gain an understanding of whether and how individuals engage with their built environment.

The existence of such policies as complete streets does not capture either their implementation or their impact on active transport. Having a policy in place does not ensure that it is fully implemented and is having an impact. Data on implementation and more research on impact that allow us to take these metrics one step further would better represent the extent to which change and/or action may occur.

Access to early childhood education represents enrollment but does not distinguish quality of educational experience. Similarly, the number of libraries per 100,000 individuals does not measure facility conditions or hours that the library is open. Some of these data are currently available and can be mined for next-generation Culture of Health metrics.

Changes in the built and social environment also make for measurement challenges. Renovation and development may cause changes that are seemingly small and difficult to capture (e.g., two-way streets may become one-way streets, new streets may be added, and others may be eliminated). This can have significant local impact but is very difficult to capture on a national level. New technologies, tools, and data collection methods are starting to capture complex interplays among the built and social environment, but scaling up to national aggregate statistics may be difficult. 


\section{Strengthening Integration of Health Services and Systems}

This chapter provides an overview of the action area related to strengthening integration of health services and systems. As in the other chapters, we provide a vision and definition for this action area, discuss factors that will move the health system toward this vision, delineate the drivers selected to represent this action area and rationale for each, and provide insight into gaps and progress toward achieving improvements in this action area. In short, this action area seeks to reimagine and redesign the health sector to place more focus on three areas: (1) improving access within and across key systems, (2) improving consumer experience and quality as consumers interact and move among systems, and (3) drawing more attention to the need for balance and integration of public health, health care, and social service systems. This chapter draws on the inputs from the environmental scan, interviews about health care system integration, and discussions with experts on issues related to this action area.

\section{Envisioning and Defining This Action Area}

The nation has a fragmented health care delivery system in which patient health care is uncoordinated across providers and pharmacies; patients access emergency departments for nonurgent care; many emergency department visits and hospitalizations are avoidable; medical services are often duplicated among various providers; medication prescribing, usage, and adherence are inconsistent; and discrepancies exist between treatment and services and evidencebased guidelines. In all, uncoordinated care costs the system five times more than coordinated patient care (Young, Saunders, and Olsen, 2010).

The vision for this action area is a coordinated, overall health system that would balance and integrate health care, public health, and social services and systems. This system would be patient- and family-centered, assessment-driven, evidence-based, and team-based to meet the needs of all ages to improve health and well-being while minimizing the caregiving burden and enhancing the caregiving capacity of families and other support persons. It would address interrelated medical, social, developmental, behavioral, educational, and financial needs to achieve optimal health and wellness outcomes provided in the context of a health team (real or virtual) that has established working relationships with families, clinicians, community partners, 
and other professionals (Hong, Siegel, and Ferris, 2014). The importance of social services (and the lack of integration with the health care system to date) may help to explain why the United States spends more on health care than other Organisation for Economic Co-operation and Development (OECD) countries but has worse outcomes (Bradley and Taylor, 2013).

Central to this vision is an expansion of how we perceive the health sector, which has traditionally been limited to provider offices, hospitals, and pharmacies. A number of societal factors point to the need to reframe this conceptualization.

First, preventable chronic conditions have skyrocketed. As of 2012, about half of all Americans lived with one or more chronic conditions, and more than a quarter had two or more chronic conditions (Centers for Disease Control and Prevention and ASCD, 2014; Ward, Schiller, and Goodman, 2014). There is a need for integrated care and delivery systems that include public health, social service, and other community organizations to support chronic disease management and prevention efforts. Examples of evidence-based practice redesign models, such as the chronic care model (Coleman et al., 2009; Wagner, Austin, and Von Korff, 1996), are moving in this direction and attempting to formalize connections among providers, patients, and their support systems (e.g., caregivers); health systems; and the larger community to increase providers' expertise and skill, educate and support patients, make care delivery more team-based and planned, and more effectively use registry-based information systems (Coleman et al., 2009). The expanded chronic care model includes elements of promoting population health so that broad-based prevention efforts, recognition of the social determinants of health, and enhanced community participation can also be part of health system teams' work on chronic disease issues (Barr et al., 2003). A number of behavioral lifestyle interventions, such as the Diabetes Prevention Program (Diabetes Prevention Program Research Group, 2002, 2009; Whittemore, 2000), are also moving toward this comprehensive approach to health by providing opportunities for communities to engage in chronic disease prevention and risk factor reduction.

Second, the population is aging. By 2060, 24 percent of Americans will be 65 and olderdouble the current older adult population (Colby and Ortman, 2015). New systematic models of healthy aging and care are needed to link transitional supports to a range of medical and community settings, such as assisted living, nursing homes, or hospice care (Institute of Medicine Committee on the Future Health Care Workforce for Older Americans, 2008). The aging U.S. population has also raised the issue of caregivers, who provide a substantial amount 
of informal health-related services in the home or other community contexts, and the burden they face as they try to balance the care and management of their parents and their children. Just under 40 million Americans (16.6 percent of U.S. adults) provide unpaid care for an adult (National Alliance for Caregiving and AARP Public Policy Institute, 2015). Unpaid caregivers provide an estimated 90 percent of long-term care, and research shows that caregiving can result in deleterious mental and physical health; a stronger, more integrated health system may alleviate some of this burden (Feinberg et al., 2011; Institute of Medicine Committee on the Future Health Care Workforce for Older Americans, 2008).

Third, there is a growing recognition that many health-related services can be provided in nontraditional settings and systems, which can help to overcome social and structural barriers. To meet the health needs of children and youth, for example, we have seen the development and proliferation of health-related services (e.g., substance abuse counseling, mental health care, dental health care) in the public health and educational systems. SBHCs, which are collaborations between an educational system and a traditional health care provider, are a major component of the nation's health care safety net that not only enable children with acute or chronic illness to attend school but also provide health and wellness through health promotion and disease prevention activities (Keeton, Soleimanpour, and Brindis, 2012). Head Start and Early Head Start— preschool to millions of disadvantaged children — have performance standards related to physical health, mental and behavioral health, and oral health and work with children, families, and staff on a range of health-promotion activities.

Fourth, key national and federal agencies now acknowledge the importance of integrating health care, public health, and social services and systems and have made recommendations to support this integration. For example, the Institute of Medicine made several agency (Health Resources and Services Administration [HRSA], CDC) and departmental (U.S. Department of Health and Human Services [HHS]) recommendations for integrating primary care and public health to improve population health. Agency-level recommendations for HRSA and CDC included linking staff, funds, and data at the regional, state, and local levels; creating common research and learning networks to foster and support integration; and developing the workforce needed to support the integration. Departmental-level recommendations (HHS) included (but are not limited to) supporting pilots that better integrate primary care and public health (such as Capability Maturity Model Integration), using the Clinical and Translational Science Awards to 
develop and diffuse research advances to applications in the community (National Institutes of Health), developing a population measure to support the integration of community-level clinical and public health data (Office of the National Coordinator), and working to develop a national strategy and investment plan for creating a primary care and public health infrastructure strong enough and appropriately integrated to enable the agencies to further the nation's population health goals (HHS-wide) (Institute of Medicine, 2012).

\section{Drivers in the Action Area of Strengthening Integration of Health Services and Systems}

We identified three drivers for this action area: access, consumer experience and quality, and balance and integration. We finalized these three as primary drivers because the literature review and stakeholder input noted that these three areas were key to improving early use of health care services that are preventive, driving more balance in health care costs (a key outcome area), and maintaining the U.S. focus on high-quality health care. Further, the balance and integration driver was specifically included to prioritize interest in balance among health care, public health, and social services, a feature also being examined globally.

\section{Access}

Access to health services and systems has been consistently linked to decreased morbidity/chronic disease burden, mortality, and health inequities; better quality of life; and overall cost savings (Institute of Medicine, 2001, 2002). Though access does not guarantee utilization of needed services, it is a prerequisite. Here, access is defined as easy entry to appropriate care through the health care, public health, or social service systems. Care refers to a wide range of health services, including preventive, primary, mental health, behavioral health, oral health and vision, specialty, prenatal, hospital, hospice/palliative, and long-term and postacute care, as well as pharmacy support, care provided in skilled nursing facilities, home health, caregiving support, and residential care in the community.

Access was selected as a driver because it is imperative that, for an integrated system to be effective, all individuals must have access to it and must be able to access it via a wide range of entry points. This entry point might include physical or mental/behavioral health, public health, or social services - in effect, creating a "no wrong door" philosophy. Furthermore, access has 
been consistently linked to decreased morbidity/chronic disease burden, mortality, and health inequities; better quality of life; and overall cost savings. If consumer experience and quality and balance and integration of the system improve, but individuals cannot access the system, we are unlikely to see long-term changes in the health of our nation.

Several factors may influence access. At the individual level, health insurance affordability and coverage has perhaps the most direct impact. Individuals without insurance are less likely to access recommended care and more likely to receive poorer quality of care and have worse health outcomes than the insured, especially among individuals who are sicker or who have chronic conditions (Institute of Medicine, 2002; McWilliams, 2009). Cultural or linguistic preferences and health literacy play a role in access to both health-related information and one's ability to navigate within and across systems of the broader health sector to access services. Low health literacy tends to be more prevalent in certain minority groups and those with low incomes and less education (National Center for Education Statistics, 2006; Wolf, Gazmararian, and Baker, 2007).

As conceptualized by Aday and Andersen, patient population characteristics (e.g., health insurance, income, and language) and delivery system characteristics (e.g., the distribution, organization, and workload of the provider labor force) impact patients' ability to access health care (Aday and Andersen, 1974). One core factor involves the extent to which service capacity is well-distributed across populations and geographies, particularly for specialty care, such as mental or behavioral health (Tomlinson et al., 2013). In addition to the distribution of providers, the types of insurance plans that they accept impact access. Fewer providers, for example, accept Medicaid, making access more difficult for lower-income individuals even if there are numerous providers in the area. The availability of social and structural supports, such as transportation assistance and case management services, are important factors that may help to overcome more traditional barriers to accessing health care.

Finally, policies and the broader decision environment influence an individual's access to services and systems. The ACA, signed into law in March 2010, put in place comprehensive insurance reforms designed to improve access to health care, strengthen consumer protections, improve quality of care, and lower health care costs. Studies have also found that expanding Medicaid and free care (reduced cost-sharing) leads to improvements in hypertension, dental health, vision, and selected serious symptoms, especially among the sickest and poorest (RAND 
Health Insurance Experiment [Keeler et al., 1985]). Beyond insurance, establishing a regular source of care facilitates continuity of care, encourages early seeking of care, and reduces delays in care or inappropriate use of resources (Henry J. Kaiser Family Foundation, 2012), which in turn improves health and lowers health care costs.

Policies to support workforce diversification or independent practice laws can also help to address provider shortages and make health care more efficient and cost-effective. For example, nurse practitioners or physician assistants may be able to adequately handle routine, preventive, or common health care needs at less cost, leaving physicians to address more complex or challenging consumer health needs. Reimbursement and funding for flexible care delivery models (e.g., mobile units, after hours, retail clinics) also impact access and include policies that expand options for service delivery to enhance access and reach, particularly to underserved populations.

Key factors influencing access are summarized in Table 8.1.

Table 8.1. Factors Influencing Access

\begin{tabular}{|c|c|}
\hline Level & Factors \\
\hline Individual & $\begin{array}{l}\text { - } \quad \text { Affordability (e.g., insurance) } \\
\text { - } \quad \text { Cultural and linguistic preferences } \\
\text { - } \quad \text { Health literacy }\end{array}$ \\
\hline Organization/community & $\begin{array}{l}\text { - Availability (e.g., provider shortage, accepting new patients) } \\
\text { - Location } \\
\text { - Social and structural supports in the community (e.g., transportation, case } \\
\text { management) }\end{array}$ \\
\hline $\begin{array}{l}\text { Decision environment/ } \\
\text { policies }\end{array}$ & $\begin{array}{l}\text { - Universal health care coverage and/or national/publicly funded health system } \\
\text { - Policies to support workforce diversification, such as credentialing } \\
\text { nonphysicians to prescribe medications and handle most primary care visits } \\
\text { (retail clinics, nurses, pharmacists, etc.) } \\
\text { - Payment and reimbursement models }\end{array}$ \\
\hline
\end{tabular}

\section{Consumer Experience and Quality}

Consumer experience relates to the ease with which consumers can move within and across systems; have a clear understanding of health and the related costs, processes, and procedures; and receive care that is high quality, respectful, and responsive to individual preferences. Consumer experience was selected as a driver because it is increasingly recognized as a critical 
component of high-quality, integrated care, yet is an often-overlooked aspect of the health system. Further, quality is linked to timely and consistent use of preventive health services (Institute of Medicine, 2001). Most individuals can recall a health care experience for themselves or a loved one that they found frustrating, difficult, or confusing. Even though the care received may have ultimately been safe and effective, many hours may have been spent on the phone with providers and payers trying to understand the situation, information may not have felt as transparent as it should be, or the patient may have perceived that his or her questions or opinions were not taken seriously enough. Addressing these shortcomings will require a concerted effort. If consumers repeatedly have poor experiences, they may be less inclined to engage in primary, preventive, or nonemergent care over time, which may negatively impact their health. It is important to note that consumers include individuals seeking health care services, as well as caregivers and individuals in one's support network who help navigate systems, support health-related decisionmaking, and provide informal care at home or in nontraditional settings (Institute of Medicine Committee on the Future Health Care Workforce for Older Americans, 2008).

Coordination among providers, caregivers, and patients is a key element of improving the consumer experience. Both the American Hospital Association, which examined user engagement (American Hospital Association, 2013), and the Agency for Healthcare Research and Quality (AHRQ), which supported the development of the Consumer Assessment of Healthcare Providers and Systems (CAHPS) surveys, are helping to focus attention on the importance of the consumer experience for an optimal health sector.

Many of the same factors that impact access are relevant to consumer experience as well. At the individual level, cultural and linguistic preferences and views on health and health care impact an individual's satisfaction with the care he or she receives. If the services and courses of care and treatment align with these core values and beliefs, the consumer will have a more positive experience. It is also important to note that this action area is not limited to the traditional health sector. The consumer experience is not driven only by interactions with a health care provider, but also by interactions with public health and social service systems. Issues of access (e.g., ease of navigation, timeliness of services) and interaction (e.g., respect for one's time or beliefs) will also impact the experience. 
At the community and institutional levels, supports in the form of decision aids, community resources, and accessible and easy-to-understand information can help individuals make personalized care decisions. Such information can empower individuals by helping them feel more in control, which can result in a more positive consumer experience overall. Individuals who believe that they have not been part of the decisionmaking process, that providers do not value their opinions, or that providers made decisions on their behalf without taking into account their personal preferences are more likely to have a negative experience, even if the care was of high quality. The consumer experience is also likely to be driven in part by how well services are integrated. Individuals who experience a more holistic approach to their health-with an integrated team that addresses physical and mental health needs, as well as other core needs around public health and social services (e.g., housing, food)—will have a more positive experience than those who work independently with each of these services and sectors, which can require more time and duplication of effort. (This holistic approach is described in more detail in the last driver, balance and integration.)

Payment and service delivery models and structures also serve as key influencers of change within the health care system and enhance the consumer experience. The United States is moving away from fee-for-service models with short time frames to thinking about health outcomes and value over the longer term (Halfon and Conway, 2013). The patient-centered medical home $(\mathrm{PCMH})$ model places primary care clinicians at the center of providing and coordinating evidence-based and patient-centered health care. Better coordination means that resources can be put to their most appropriate use, which improves quality and outcomes of patient care while decreasing costs (Agency for Healthcare Research and Quality, 2013; Friedberg et al., 2009; Rittenhouse, Thom, and Schmittdiel, 2010; Stange et al., 2010). The accountable care organization (ACO) model, defined as a "provider-run organization in which the participating providers are collectively responsible for the care of an enrolled population, and also may share in any savings associated with improvements in the quality and efficiency of the care they provide" (Henry J. Kaiser Family Foundation, 2012), adds collective clinician accountability for the overall quality and cost of care and direct patient involvement into the ACO governance (Berenson and Burton, 2012; Isaacs, 2012; RTI International, 2011). Of note, the ACO model is relevant for the access driver as well because ACOs provide strong incentives to eliminate historical access barriers to specialty care for patients with chronic and multiple health 
conditions. While ACOs show significant promise, many unanswered questions remain, including whether they will be effective at achieving their anticipated benefits of improved care and healthier populations at reduced cost. However, as different models and approaches toward developing and implementing ACOs are studied, and their effectiveness examined over time, lessons learned and promising practices may be identified (McCarthy et al., 2014). These findings may help to strengthen the effectiveness of ACOs.

It is important to note that while this action area does not explicitly call out more traditional measures of high-quality care, they are viewed as fundamental. Care and health-related supports received within the public health, mental and behavioral, social service, and health care systems should incorporate existing frameworks of quality assessment with care that is safe, equitable, effective, accessible (e.g., hours of operation), timely and efficient, affordable, patient-centered, and culturally and/or linguistically responsive, regardless of setting (Institute of Medicine, 2001).

Key factors influencing the consumer experience and quality driver are listed in Table 8.2.

Table 8.2. Factors Influencing Consumer Experience and Quality

\begin{tabular}{|c|c|}
\hline Level & Factors \\
\hline Individual & $\begin{array}{l}\text { - } \quad \text { Cultural and linguistic preferences } \\
\text { - } \quad \text { Family views of health } \\
\text { - } \quad \text { Prior health system experiences }\end{array}$ \\
\hline Organization/community & $\begin{array}{l}\text { - } \quad \text { Patient decision aids } \\
\text { - } \quad \text { Inclusion in the decisionmaking process } \\
\text { - Holistic approach to health }\end{array}$ \\
\hline Decision environment/policies & - Models of health care delivery that emphasize quality and efficiency \\
\hline
\end{tabular}

\section{Balance and Integration}

Balance specifically refers to the balance between prevention/promotion and acute/chronic care services, and integration refers to integration across public health, mental/behavioral health, social service, and health care systems (e.g., data- and information-sharing, cost/financing models, shared risk). This includes integration of entities that fall within a given system (e.g., hospital, primary care, pharmacy in the health care system) but also entails integration across systems (e.g., public health and health care) and across jurisdictions or levels (e.g., local, state, national). 
Balance and integration were selected as a driver because they highlight the extent to which the country values or prioritizes health (as opposed to emphasizing recovery from illness) and the extent to which these traditionally separate health-related sectors are operating as a unified system. Balance and integration occur when all players involved in an individual's care (within and across systems, including the individual and his or her caregivers) have the motivation and means to work together to support that individual's health. Moving toward a more balanced and integrated health system is likely to result in improved population health because precursors to more serious illnesses are identified and addressed earlier, quality of care is improved (e.g., through real-time sharing of important health information), and cost savings are likely to result to individuals (e.g., addressing illness through lifestyle changes rather than invasive procedures) and sectors (e.g., through shared resources). This driver is also important from the perspective of payment reform in that provider groups are increasingly becoming responsible for the health of their patients. There is a growing recognition that this will require tackling many of the "upstream" causes of poor health that public health and social service sectors can help to address (Martin and Luoto, 2015). The integration of public health, social service, and health care systems can improve the efficiency and quality of care delivered and improve care transitions, which can lead to reduced hospital readmissions, decreased health costs, and improved consumer experiences (Institute of Medicine, 2012, 2014a). System-level linkages can support secondary and tertiary prevention (Patterson and Chambers, 1995) of diagnosed illness, help ensure continuity of care by providing case management services, and facilitate access by patients and/or their support network to health-related services in a variety of settings that are tailored to personal preferences and health needs.

There are several models of such linkages that can inform movement in this direction, including community health worker models — systems that use frontline public health workers who are trusted members of the community to serve as liaisons between health and social services and the community (definition adapted from American Public Health Association, 2009). Care management/hot-spotting models also provide insight into ways to achieve balance and integration. For example, the Camden Coalition of Healthcare Providers uses a health information exchange (shared clinical data among health care systems) to identify high utilizers of care and provide intensive social supports, which has resulted in better patient outcomes and system cost savings. Furthermore, Stakeholder Health (a group of 43 mission-driven health care 
systems) addresses nonclinical needs that are linked with health outcomes to prevent vulnerable patients from being readmitted to the hospital (Institute of Medicine, 2014b). Scalable interventions, such as the Care Transitions Model, teach complex patients self-management skills supported by a coach to ensure that patient needs are being met during the transition from hospital to home, resulting in reduced readmissions and cost savings (Coleman et al., 2006). In all, these programs and others (e.g., Health Leads) are trying to connect the health care system to social determinants of health to improve overall population health and well-being.

In addition to these various models of interaction between and across sectors, true integration will require infrastructure and data-sharing to the extent allowable by law. The proliferation of electronic health records (EHRs, sometimes called electronic medical records [EMRs]) and related technologies will be a key influence. Health information technology (HIT) has the potential to improve care quality and health outcomes as well as the consumer experience. Several studies have demonstrated the benefits of EHRs in clinical settings, noting cost savings, improved care, better patient outcomes, and high patient satisfaction (American Telemedicine Association, 2015). For example, studies have found that EHRs with clinical decision support reduce the number of adverse drug events by 30 to 84 percent (Ranji, Rennke, and Wachter, 2013). Other components of HIT that may substantially improve quality of care are clinical data exchange, which is the ability to exchange patient health information among health care organizations, and public health data exchange, which links public health with clinical data to improve ongoing community health surveillance. In a fragmented health system, information is often missing, and standards for these data exchanges are not implemented well. Personal health records may also incorporate patient decision aids in making critical health care decisions, taking into account their personal preferences (Rudin, 2010; Rudin et al., 2011). However, to ensure efficiency and equity of the health and social services systems, public health and health care must work together to bridge the digital divide for improved coordination and to support traditionally disadvantaged groups that may have less web access than others (Saleem et al., 2011). Related to this, the Institute of Medicine recently encouraged the incorporation of social and behavioral domains in EHRs (Institute of Medicine, 2014a).

Given this literature, key factors influencing this driver are identified in Table 8.3. 
Table 8.3. Factors Influencing Balance and Integration

\begin{tabular}{|c|c|}
\hline Level & Factors \\
\hline Individual & - $\quad$ Participation in EMR or HIT initiatives \\
\hline Organization/community & $\begin{array}{l}\text { - Global medical/health record that enables providers to know medical and } \\
\text { - } \quad \text { Focial needs } \\
\text { to support referrals } \\
\text { - Workforce and training of personnel (care management, navigators, liaisons) } \\
\text { who can link the systems }\end{array}$ \\
\hline $\begin{array}{l}\text { Decision environment/ } \\
\text { policies }\end{array}$ & $\begin{array}{l}\text { - Incentives for health care systems (and social service/public } \\
\text { - } \quad \text { Mealth/community-based organizations) to have integration } \\
\text { population health outcomes } \\
\text { - Policies that link staff, funds, and data at regional, state, and local levels }\end{array}$ \\
\hline
\end{tabular}

To achieve the vision of this action area, critical changes will be required across the broader health system. Namely, systems that afford linkage and sharing of information need to be in place. To improve access, new models of service delivery must be tested, including ways to use a broader and more diverse health care workforce. Further, opportunities to promote health and deliver health care will continue to extend well past traditional settings to mobile, home-based, and retail sites. These models need to be rigorously assessed and integrated into service delivery systems. To enhance consumer experience and create more balance, the linkage and coordination among the broader health care and public health systems need further emphasis, particularly among prevention, acute care, transitional care, and social services.

\section{Measures}

The following measures were identified as capturing key aspects of each driver. We describe each measure in further detail below and then summarize them in Table 8.4.

\section{Access Measures}

Four measures were selected to capture access to four key types of health care and services: public health services, traditional health care, mental health care, and dental care. Broad access to each of these is necessary to achieve a balanced system.

Percentage of population served by a comprehensive public health system. A strong public health system is critical to the health and safety of the residents it serves. Public health plays a 
number of key roles at the state and local levels, including assessment, policy development, assurance, and equity. A public health system is considered comprehensive in scope if it "generally perform[s] more than two-thirds of the activities in each of the three IOM [Institute of Medicine] domains of assessment, policy development, and assurance" (Mays et al., 2010). This measure helps to capture the adequacy of the nation's public health protections, which include activities to monitor community health status, investigate and control disease outbreaks, educate the public about health risks and prevention strategies, prepare for and respond to natural disasters and large-scale health emergencies, and enforce laws and regulations designed to protect health, such as those concerning tobacco exposure, food and water safety, and air quality (Institute of Medicine, 1998; Mays and Hogg, 2015).

Although the data suggest that the percentage of the population covered by a comprehensive public health system has been stable since 2006, it is important to note that there have been some changes over time to the sample to be more inclusive. The first wave included health departments serving 100,000 or more individuals; health departments serving smaller geographic areas were added in 2006 and 2012, which precludes direct comparisons across waves. However, trends over time for larger communities can be assessed, and it is expected that future data collection efforts will allow for comparisons over time for smaller communities as well.

Percentage of adults with stable health insurance. In the past, much attention has been placed on the fraction of the population that is uninsured, and this will likely continue to be tracked by many organizations. For a Culture of Health, we believe stability of insurance will be particularly important to maximize health and well-being. Due to changes in employment and income, many could lose coverage temporarily or have to change types of coverage. Both situations could hamper a person's ability to have continuous access to care and their ability to stay with their preferred provider over time.

This measure includes the percentage of the population ages 18-64 experiencing no change in type of health insurance coverage at any time in the last year. The measure excludes changes in health insurance within a particular type (for example, switching from one marketplace-based plan to a cheaper marketplace plan).

Literature from the past year suggests significant declines in those who are uninsured (Carman, Eibner, and Paddock, 2015; Collins, Rasmussen, and Doty, 2014; Long, Karpman, et al., 2014; Long, Kenney, et al., 2014; Sommers et al., 2014), but because the ACA leads to many 
different potential sources of insurance coverage (employer, marketplace, Medicaid), changes in income and employment during the year may still lead to changes in the type of insurance coverage or periods with no coverage. As noted, unstable coverage could hinder consistent access to the same provider, especially among those whose insurance policies have limited networks of providers.

Percentage of adults with a mental or substance use disorder who reported receiving treatment in the past year. Mental health and substance use disorders are among the top conditions that cause disability and carry a high burden of disease in United States, resulting in significant costs to families, employers, and publicly funded health systems. By 2020, mental and substance use disorders will surpass all physical diseases as a major cause of disability worldwide. The Substance Abuse and Mental Health Services Administration (SAMHSA) and its partners (the Institute of Medicine, the National Institute of Mental Health, and the National Research Council) have documented evidence-based practices to improve outcomes for these conditions, especially through prevention and early intervention. Despite the impact of mental/behavioral health conditions, people who suffer from mental and substance use disorders may not be getting treatment because of a lack of affordability of reimbursable services, stigma, or lack of awareness of these disorders.

The composite measure of mental and behavioral health needs that have been met provides information about the proportion of Americans who report alcohol and drug abuse or dependence and/or any mental health illness and who have received treatment for their condition. This measure is important to include because mental/behavioral health care needs are often underrecognized, stigmatized, and not always covered by health insurance (though this is changing with the Mental Health Parity and Addiction Equity Act and ACA provisions for requiring coverage of mental health and substance use disorders). In addition, individuals with untreated mental/behavioral conditions often account for a large proportion of health care costs (e.g., they are high utilizers of emergency department care). This measure also speaks to the need for integrating mental/behavioral health with primary care. In many cases, these conditions prevent individuals from functioning in or being productive members of society (e.g., going to school or work). This measure would be of interest not only to mental health providers, which include providers in social/human service agencies, public health departments, and health care institutions, but also employers, schools, and those in the criminal justice system. 
The measure includes the following indicators:

- percentage of adults 18 and older with a mental health illness or substance use disorder who reported receiving treatment in the past year

- past-year alcohol or drug treatment among those who reported past-year substance abuse or dependence only

- any mental illness treatment in the past year among those with mental illness only (the survey uses a prediction model to assess minimal, moderate, or serious mental illness). Substance abuse and dependence as well as mental illness are assessed using a series of questions that are predictive of these disorders. The survey used for this measure, the National Survey on Drug Use and Health (NSDUH), contains several series of questions to assess these disorders: Substance abuse and dependence are assessed using DSM-IV, while mental illness is assessed using a scale developed by Kott et al. (2013) for use in NSDUH based on the World Health Organization Disability Assessment Schedule (WHODAS) and Kessler-6 (K6).

With the health care environment changing around mental/behavioral health payment, the hope is that more people will have access to needed services. Health care reform and the Mental Health Parity and Addiction Equity Act both expand benefits and coverage for mental health and substance use disorders and should result in more people with these health needs being able to afford these services and get treatment. According to a 2013 ASPE Issue Brief (Assistant Secretary for Planning and Evaluation, 2013), 32.1 million Americans will gain access to coverage that includes mental health and/or substance use disorder benefits, and an additional 30.4 million Americans who currently have some mental health and substance abuse benefits will benefit from federal parity protections that seek to ensure that coverage for mental health and substance abuse care is on par with that for medical and surgical care. This will impact 62.5 million Americans.

There is evidence that increased access to mental/behavioral health services from parity laws results in increased treatment rates and follow-up, but there is also research on children that suggests that parity laws do not improve receipt of mental health services. A recent report by SAMHSA (2015) demonstrates an increase in insurance coverage for young adults since the ACA extended dependent care coverage. This increase in insurance coverage has led to higher rates of mental health treatment but has had little impact on substance use treatment for this age group. 
Percentage of adults who report a dental visit in the last year. Proper dental hygiene and regular dental visits are important to maintaining healthy teeth and gums. There is also a strong link between oral health and overall health. Good oral health and regular visits are particularly important during pregnancy because hormonal changes can increase the risk of gum disease, which can affect the health of the fetus. Additionally, those with lost, broken, or discolored teeth report shame and embarrassment, which can affect mental health, well-being, and economic opportunities and outcomes. Despite its importance, dental care often does not receive the same attention as other types of health care; we include it here to highlight its significance (Petersen et al., 2005; Sheiham and Watt, 2000).

This measure is a driver for health because regular dental visits can help to identify oral health problems early. Dental visits also help prevent many oral problems from developing and may detect other diseases, such as cancers in the mouth (Seymour, Preshaw, and Steele, 2002).

We foresee several possible channels to increase use of dental care. If educational campaigns and other efforts are successful at getting people to see a dentist, this measure will be able to capture that. This measure may also increase as dental coverage and the number of dental providers grow (e.g., in dental professional shortage areas).

It is expected that, across the United States, there will be some improvements in the number reporting regular dental visits in the short term and greater improvements in the medium term. In the long run, we expect reductions in disparities in dental visits, as well as linkages with reductions in avoidable dental problems.

\section{Consumer Experience and Quality Measures}

Two measures were included to capture consumer experience and quality. As stated above, we do not call out traditional measures of quality but rather focus on the availability of a particular type of quality care: ACOs. Consumer experience is measured using a newly developed comprehensive measure of consumer perceptions of their experience with providers.

CAHPS summary measure of consumer experience across ambulatory, hospital, and home health care settings at the state level. Improving consumers' experience of health care is a central goal of this action area. This measure provides a summary of the consumer experience at the state level across a variety of providers. Over the past 20 years, the CAHPS project funded by AHRQ has developed products that have become the national standard for collecting 
information, reporting, and evaluating patients' experience of care. CAHPS surveys are administered in different health care settings (e.g., primary care or specialty physician offices, hospitals, nursing homes, at home through home health services, hospices). This measure utilizes data from three CAHPS data sets to create a composite measure of the consumer experience of care in each state. Data are combined from HCAHPS (hospital), ACO CAHPS, and MCAHPS (Medicare), each of which is publicly available and representative of its target population. These data are aggregated at the state level.

The information that goes into the index comes from nationally representative surveys of the experience of adult patients in outpatient (Medicare CAHPS), inpatient (hospital CAHPS), and home health care settings (home health CAHPS). Multiple domains contribute to the assessment of experience in each setting, including but not limited to the timeliness of care, provider communication, and access to services. The index combines scores across all these domains into a single numeric summary of experience. According to the index score, each state is assigned a rating from 1 to 5 stars, with 1 indicating the poorest possible performance and 5 the best.

CAHPS measures have undergone extensive psychometric analyses (Price and Elliott, 2014). Development and validation of individual CAHPS surveys are available in the literature and from AHRQ. RAND has presented the methods for developing and validating the measure (Kovalchik et al., under review).

Percentage of population whose health care provider is part of an accountable care organization. Payment and service delivery models and structures such as ACOs and PCMHs that reward high-quality integrated care can also play a key role in improving consumers' experience. One of the main ways the ACA seeks to reduce health care costs and deliver better patient care is by encouraging doctors, hospitals, and other health care providers to form networks that coordinate patient care and become eligible for bonuses when providers deliver that care more efficiently (by keeping people healthy and out of the hospital). ACOs are one of the primary and most pervasive examples of this type of coordinated health care system, in which a network of doctors and hospitals share financial and medical responsibility for a patient's health. If successful, the U.S. health care sector should see lower health care costs, better patient care, fewer people with disabling or chronic medical conditions, and more people living longer with better quality of life. A high-performing health care delivery system, such as an ACO, will improve the quality of care that consumers receive, and more integrated networks 
will help to improve the experience, as consumers will not be responsible for the coordination of their care across diverse providers.

This measure is based on data collected by Leavitt Partners, which defines an ACO as "a group of health care providers that agree to oversee the provision of health care services to a defined population with responsibility to reach certain quality benchmarks and some financial responsibility for the total cost of care for the population" (Muhlestein, 2013). As of May 2014, 20.5 million people (6.5 percent of the U.S. population) were covered by ACOs, which have grown rapidly since 2010 . This measure is mainly a driver of health care redesign and reimagining. It may also be an outcome if ACOs are found to be the best model of health care delivery (Muhlestein, 2014). Provisions in the ACA encourage the growth of ACOs and other new payment and care delivery models that seek to tie provider reimbursements to quality metrics and reductions in the total cost of care for an assigned population of patients.

ACA provisions enable ACOs or new payment and care delivery models that seek to tie provider reimbursements to quality metrics and reductions in the total cost of care for an assigned population of patients. In other words, ACOs can facilitate and incentivize collaboration across various providers and organizations. The verdict on ACOs' impact varies, but this is the direction of health care reform efforts to meet the triple aim of improving care quality and health and lowering costs as described by Berwick, Nolan, and Whittington (2008).

We expect that in the short term, more individuals will receive care from high-performing ACOs. In the medium term, more individuals will receive care from a high-performing health care delivery system (which may not be limited to ACOs). In the long term, more people will receive care from a high-performing health delivery system, which would include such sectors as public health or social/human service agencies.

\section{Balance and Integration Measures}

Four measures were selected to reflect integration between medical care providers, integration between hospitals and other types of organizations with a focus on primary care, and balance between health care and social services.

Percentage of physicians who share data with other providers, including physicians and hospitals. Coordination of care between providers helps to ensure quality of care and safety for patients. One important way to do so is through the coordination and sharing of information in 
EHRs. This measure captures how well EHRs contribute to integrated care across providers. Development of infrastructure to support integration of care is necessary to achieving fully integrated care and could also spur integration.

O’Malley et al. (2010) conducted an extensive assessment of EHRs' impact on care coordination. They found that clinicians needed to help with communication between settings, that current records do not support care coordination planning, and that care coordination processes need to evolve. National policy has been pushing for the transition to EHRs for some time, and further coordination of care is central to the meaningful use Stage 2 criteria.

While we expect care coordination to grow over time because of meaningful use requirements, it is a relatively new concept, and the sensitivity to change is not known. However, Furukawa et al. (2014) found that 38.9 percent of physicians share information with other providers, suggesting room for improvement.

Percentage of hospitals that have a collaboration or alliance with one or more organizations in each of these categories: local government, state agencies, and other community-based agencies. Recognizing the paradigm shifts toward population health, hospitals and health care systems are increasing leadership engagement, collaborating with community partners, and expanding their scope of services to address nonmedical factors that influence the health status of their communities. Partnerships with various types of community organizations speak to a hospital's commitment to improving the health of its community in a balanced way.

This measure is a driver for greater integration of health and health care systems, which is likely to result in improved population health. "Financial and programmatic constraints . . . at all levels in the 1990s required health departments to aggressively seek partnerships, coalitions, and shared resources wherever possible to achieve objectives, rather than rely on hierarchical, bureaucratic approaches that may have worked in a different political and budget environment. Public school systems, governmental agencies in non-health areas (e.g., justice, welfare, housing), churches, hospitals, institutions of higher learning, and the wide array of communitybased nonprofit organizations exemplify the types of partners with which innovative [hospitals and governmental agencies] have collaborated" (Baker et al., 1994).

Number of states that have full practice laws and regulations for nurse practitioners. Nurses play an important role in advancing care. While the majority practice in acute care settings, primary care and prevention are central drivers in a transformed health care system. Nurses could 
play an even more vital role in improving the quality, accessibility, and value of health care and, ultimately, health in the community.

The current landscape also points to a need to focus on primary care. There is concern over an adequate supply of primary care providers, and demand for primary care is expected to grow as millions more Americans gain insurance through implementation of the ACA. Additionally, many ACA provisions focus on improving access to primary care, offering further opportunities for nurses to play a role in transforming the health care system and improving patient care. This points to the need to expand practice laws and regulations for nurse practitioners (NPs) (Institute of Medicine, 2010).

This measure reflects state practice and licensure laws that provide NPs with the authority to evaluate patients; diagnose, order, and interpret diagnostic tests; and initiate and manage treatments - including prescribe medications - under the exclusive licensure authority of the state board of nursing. In May 2015, 19 states had full practice laws, which the Institute of Medicine and the National Council of State Boards of Nursing recommend for all states.

Ratio of annual social spending to annual health expenditures in the United States. The "health care paradox" is that the United States is spending more per person on health care than any other industrialized country but ranks among the lowest in many action areas of health. The nation spends, as a percentage of gross domestic product, about half of what some industrialized counties spend on social services (e.g., housing, employment training, unemployment benefits, senior care, and family support services). The ratio of social spending to health spending in the United States is less than 1 to 1 , while the average among other OECD countries is 2 to 1.

The work of Bradley et al. (2011) indicates that, across countries, the ratio of social to health spending is significantly associated with better health outcomes: lower rates of infant mortality, low birth weight, and premature deaths, as well as longer life expectancy (nonsignificant for maternal mortality). A 2011 report by the National Research Council confirmed a large international "mortality gap" among adults age 50 and older. The U.S. health disadvantage cannot be attributed solely to the adverse health statuses of racial or ethnic minorities or poor people because recent studies suggest that even highly advantaged Americans may be in worse health than their counterparts in other countries. The Panel on Understanding Cross-National Health Differences Among High-Income Countries was charged with examining whether the U.S. health disadvantage exists across the life span, exploring potential explanations, and 
assessing the larger implications of the findings (National Research Council, 2011). The panel's analysis compared health outcomes in the United States with those of 16 comparable highincome or "peer" countries: Australia, Austria, Canada, Denmark, Finland, France, Germany, Italy, Japan, Norway, Portugal, Spain, Sweden, Switzerland, the Netherlands, and the United Kingdom. It uncovered a strikingly consistent and pervasive pattern of higher mortality and inferior health in the United States, beginning at birth.

Table 8.4 summarizes the measures that represent this part of the action framework.

Table 8.4. Strengthening Integration of Health Services and Systems: Measures by Driver

\begin{tabular}{|c|c|c|}
\hline Driver & Measure & $\begin{array}{c}\text { What It Means to the Action Area/ } \\
\text { Culture of Health Overall }\end{array}$ \\
\hline Access & $\begin{array}{l}\text { Percentage of population served } \\
\text { by a comprehensive public } \\
\text { health system }\end{array}$ & $\begin{array}{l}\text { Highlights the proportion of individuals who } \\
\text { reside within an area (often referred to as a } \\
\text { catchment area) and, as a result, have access to a } \\
\text { stronger, more comprehensive public health } \\
\text { department. Transforming health and health care } \\
\text { systems requires thinking about public health as a } \\
\text { core health resource for the community. However, } \\
\text { some public health departments are more } \\
\text { comprehensive in their activities than others. }\end{array}$ \\
\hline Access & $\begin{array}{l}\text { Percentage of adults with stable } \\
\text { health insurance }\end{array}$ & $\begin{array}{l}\text { Demonstrates continued access to health care and } \\
\text { is superior to measures of insurance coverage that } \\
\text { do not capture churn. Access to care for many } \\
\text { requires insurance, but changing insurance may } \\
\text { reduce individuals' ability to have access to the } \\
\text { same providers over time. Stable health insurance } \\
\text { supports long-term engagement with the health } \\
\text { system. }\end{array}$ \\
\hline Access & $\begin{array}{l}\text { Percentage of adults with a } \\
\text { mental or substance use } \\
\text { disorder who reported receiving } \\
\text { treatment in the past year }\end{array}$ & $\begin{array}{l}\text { Addresses utilization of behavioral health, but } \\
\text { access is a prerequisite. Getting a sense of } \\
\text { prevalence of these conditions and utilization of } \\
\text { treatment may provide insight into how many } \\
\text { people who need a mental or behavioral health } \\
\text { care service are actually seeking care. }\end{array}$ \\
\hline Access & $\begin{array}{l}\text { Percentage of adults who } \\
\text { reported a dental visit in the last } \\
\text { year }\end{array}$ & $\begin{array}{l}\text { Captures the percentage of the population who } \\
\text { have access to and are engaging with the oral } \\
\text { health sector on a fairly regular basis. Dentists are } \\
\text { an important part of a comprehensive health } \\
\text { sector. Though this measure is focused on } \\
\text { utilization, access is a key component of that. }\end{array}$ \\
\hline
\end{tabular}




\begin{tabular}{|c|c|c|}
\hline Driver & Measure & $\begin{array}{l}\text { What It Means to the Action Area/ } \\
\text { Culture of Health Overall }\end{array}$ \\
\hline $\begin{array}{l}\text { Consumer } \\
\text { experience and } \\
\text { quality }\end{array}$ & $\begin{array}{l}\text { CAHPS summary measure of } \\
\text { experience across ambulatory, } \\
\text { hospital, and home health care } \\
\text { settings at the state level }\end{array}$ & $\begin{array}{l}\text { Combines CAHPS data across multiple settings to } \\
\text { create a comprehensive measure of consumer } \\
\text { experience of care at the state level. Redesigning a } \\
\text { health sector must take into account consumer } \\
\text { experience within and across health systems. It is } \\
\text { an essential component of health care quality, and } \\
\text { CAHPS is the national standard for collecting } \\
\text { information, reporting, and evaluating patients' } \\
\text { experience of care. The surveys are administered } \\
\text { in different health care settings (i.e., ambulatory, } \\
\text { hospital, home health), providing an opportunity } \\
\text { to potentially examine consumer experience across } \\
\text { one or more settings. }\end{array}$ \\
\hline $\begin{array}{l}\text { Consumer } \\
\text { experience and } \\
\text { quality }\end{array}$ & $\begin{array}{l}\text { Percentage of population whose } \\
\text { health care provider is part of } \\
\text { an ACO }\end{array}$ & $\begin{array}{l}\text { Shows the extent to which populations are being } \\
\text { served by an ACO model focused on higher-value } \\
\text { care. ACOs provide access to new payment and } \\
\text { health care delivery models that are supposed to } \\
\text { provide better care at lower cost and result in } \\
\text { better health, though the evidence continues to } \\
\text { emerge. }\end{array}$ \\
\hline $\begin{array}{l}\text { Balance and } \\
\text { integration }\end{array}$ & $\begin{array}{l}\text { Percentage of physicians who } \\
\text { share data with other physicians } \\
\text { and hospitals }\end{array}$ & $\begin{array}{l}\text { Provides a first look at how well physicians } \\
\text { integrate care with other medical providers, using } \\
\text { EHR and health IT technology. }\end{array}$ \\
\hline $\begin{array}{l}\text { Balance and } \\
\text { integration }\end{array}$ & $\begin{array}{l}\text { Percentage of hospitals that } \\
\text { have a collaboration or alliance } \\
\text { with one or more organizations } \\
\text { in each of these categories: } \\
\text { local government, state } \\
\text { agencies, and other community- } \\
\text { based agencies }\end{array}$ & $\begin{array}{l}\text { Indicates the types of partners a hospital has. To } \\
\text { transform the health and health care systems, key } \\
\text { health entities, such as hospitals, should be } \\
\text { integrated with other community resources that } \\
\text { also impact health. }\end{array}$ \\
\hline $\begin{array}{l}\text { Balance and } \\
\text { integration }\end{array}$ & $\begin{array}{l}\text { Number of states that have full } \\
\text { practice laws and regulations } \\
\text { for nurse practitioners }\end{array}$ & $\begin{array}{l}\text { Removing scope-of-practice barriers is not only } \\
\text { expected to alleviate access barriers but also to } \\
\text { help balance points of care so that primary, } \\
\text { preventive, or routine medical needs may be able } \\
\text { to be met in a wider range of settings. }\end{array}$ \\
\hline $\begin{array}{l}\text { Balance and } \\
\text { integration }\end{array}$ & $\begin{array}{l}\text { Ratio of annual social spending } \\
\text { to annual health spending in the } \\
\text { United States }\end{array}$ & $\begin{array}{l}\text { Indicates the extent of the balance (or lack thereof) } \\
\text { between social service spending and health } \\
\text { spending. }\end{array}$ \\
\hline
\end{tabular}

\section{Limitations}

While these measures capture the essence of the drivers in this action area, there are important considerations moving forward that will likely alter the measurement approach over time. 


\section{Moving beyond traditional measures of quality, such as the IOM domains of quality}

assessment (IOM, 2001), to capture elements of consumer experience will not be easy. While the CAHPS consumer experience surveys are a start, it will be important to consider more comprehensive measures of consumer engagement in health care decisionmaking. The current CAHPS measure captures the consumer experience in the hospital, ambulatory, and home health care settings, but future iterations could reflect experience in additional settings. Consumer experience across the entire health system could signal advancement in understanding patient interaction and satisfaction.

Better understanding is needed of the value and benefits of new health care structure and delivery models, such as ACOs and PCMHs. Though our current measure captures the proportion of a population covered by an ACO, it is not yet clear whether the ACO is the "right" or "best" model to achieve high-quality, efficient care that results in good outcomes and a positive consumer experience. Our current measure assumes that better distribution of these models is beneficial, but we do not yet have the robust evidence base to support that assertion. Monitoring the growth and quality of these models, as well as where they confer value, will be important as we integrate these measures into the Culture of Health strategy.

Health information technology will be important for capturing integration, but our measure does not capture the signal we need for health care system efficiency. The current measure captures the extent to which data were shared with individuals inside or outside the organization. Ideally, however, the country will need to push beyond the mere presence or use of EHR or HIT to include meaningful use data. A more forward-leaning measure would assess the extent to which data are captured in the EHR on consumer lifestyle, social circumstances, and social service needs in order to facilitate linkages with other social systems and provide an individual with comprehensive care, though this is not currently captured in any systematic way.

Currently, measures of system linkages, coordination, or integration are lacking. Given the reciprocity implied in the Culture of Health model and the fluidity with which consumers use several systems to address health, it is essential to have a measure of coordination or integration with nontraditional health systems - particularly social services. However, this is a challenging measure to identify — in part, because there appear to be few, if any, measures of linkages across systems and none that have been well validated at the national level. For Culture of Health efforts, this measure may need to be derived locally at first. 


\section{Improving Population Health, Well-Being, and Equity}

This chapter presents an overview of the Culture of Health outcome measures, which are intended to track improvements in population health and well-being, as well as the pursuit of equity in achieving these outcomes. If there is progress in each of the four action areas of the Culture of Health framework, the result should be improvements in overall population health and well-being. The definition of health that we use is intentionally broad, and it is important that this definition be fully taken into account as measurement of a Culture of Health evolves. As such, the selected outcomes consider the full continuum of health and well-being, including the following outcome areas: (1) enhanced individual and community well-being, (2) managed chronic disease and reduced toxic stress, and (3) reduced health care costs. In the next sections, we describe the vision behind the outcome areas, define these areas, and offer illustrative measures for each one.

\section{Envisioning and Defining Outcomes}

The selected outcomes of a Culture of Health are intended to account for the broad definitions of health and well-being, so they require indicators that are more complex than the simple indicators that traditionally track health and clinical outcomes. The overall goal of the outcomes for the Culture of Health action framework is to capture system-level change and potentially complex interactions within and across the four action areas. As such, the outcome areas described in the next section are intended to summarize what a Culture of Health yields over time.

Widespread dissemination of a Culture of Health in the United States is expected to result in improvements in population health and well-being occurring equitably across communities. Health and well-being are interrelated, and sometimes inextricably so, as exemplified by the WHO definition of health: "a state of complete physical, mental and social well-being and not merely the absence of disease or infirmity" (World Health Organization, 1948). But some health initiatives, such as Healthy People 2020 and the HHS Strategic Plan, are now making the effort to distinguish well-being from health in their measures. The definition of well-being as linked to the establishment of a Culture of Health is the realization of a population that is flourishing. In 
their conceptual framework for defining well-being, Huppert and So (2013) describe flourishing as "the experience of life going well" and as something that can itself be nurtured and grown, as opposed to a state that is reached. Having greater levels of well-being is beneficial for both individuals and society overall. Well-being is associated with effective learning, productivity and creativity, good relationships, pro-social behavior, and good health and life expectancy (Chida and Steptoe, 2008; Diener et al., 2010; Dolan, Peasgood, and White, 2008; Huppert, 2009; Lyubomirsky, Sheldon, and Schkade, 2005).

A Culture of Health is also predicated on the idea that the health and well-being of individuals is interconnected, which suggests that community-level investments in health and well-being are a key mechanism to influence individual-level health. We should expect to see advancements in the attitudes and practices of individuals, organizations, and communities around health promotion and policy. Equally important in achieving equity in these outcomes is that the access to and use of health-related resources does not significantly differ due to income level, age, race/ethnicity, region, or culture. There are well-documented disparities along these factors, indicating that the United States has not yet achieved a Culture of Health. Low-resourced communities often have fewer parks that are safe to use (Babey, Hastert, and Brown, 2007; Sallis et al., 2011) and fewer venues for physical activity (Panter and Jones, 2008). Residence in lowincome neighborhoods is associated with lower levels of well-being than in wealthier neighborhoods (Ludwig et al., 2012). Research has shown that higher neighborhood walkability levels are associated with more walking/biking (Built Environment Health Research Group at Columbia University, n.d.), but because more than half of blacks live in poor neighborhoods (Bishaw, 2014), there are likely to be significant racial disparities in walkability. In an urban study using nontraditional walkability measures, poor communities were found to have fewer and less clean streets and more public safety issues than communities that were not poor (Neckerman et al., 2009). Looking at neighborhood racial distribution, predominantly black neighborhoods were more likely to have poor walkability indicators - uneven sidewalks, obstructions, and physical disorder - when compared to predominantly white neighborhoods (Kelly et al., 2007). 


\section{Outcome Areas and Definitions}

Based on the environmental scan of where the nation is in terms of achieving RWJF's ten principles of a Culture of Health, we identified three outcome areas in which we will track observed impact if progress is made in the four action areas and the related drivers. Given that the drivers are in the action areas, we use the term "outcome areas" to distinguish from "drivers."

\section{Enhanced Individual and Community Well-Being}

The action framework for establishing a Culture of Health is unique, given the emphasis on well-being. There are several reasons why this outcome area is included. First, the establishment of a Culture of Health is, by definition, a transformation in the way society thinks about health, a result that can only happen when complex, system-level change occurs. Therefore, looking at only traditional health outcomes, particularly ones that track specific disease conditions, is not comprehensive enough in scope. Second, well-being acknowledges imperfect health (or that illness can exist) - that people with chronic conditions or other illnesses can still thrive and still lead a healthy life. One of the anticipated goals of establishing a Culture of Health is to have a lower incidence of preventable disease. However, some conditions are not preventable, and about half of all Americans have at least one chronic condition (Ward et al., 2014). The Culture of Health action framework focuses on what is preventable and on improving care management of existing and nonpreventable conditions so that all Americans can live their best lives.

Ideas around measuring well-being have been advanced since the 1960s. The direct measurement of experienced well-being has made an important contribution to this area, with academics and think tanks calling for "National Accounts of Well-Being" (e.g., Kahneman et al., 2004; Shumacher Center for a New Economics, 2011). Early pioneers of well-being measurement include the Jacksonville Council on Citizen Involvement quality of life indicators project in Florida, which launched in 1985. More recently, policymakers are using well-being data to drive policy decisions. One example of a government beginning to integrate well-being into policymaking is the City of Santa Monica, which developed and launched its Wellbeing Index in April 2015 to assess the well-being of its residents, using survey, administrative, and social media data (City of Santa Monica, 2015). Santa Monica intends to use this information to set priorities and make decisions in ways that will improve residents' well-being. 
Research distinguishes between individual and community well-being because the measurement of each differs. Both subjective and objective measurements of well-being are possible. Individual well-being measures are generally operationalized through a range of action areas, including life satisfaction (Diener and Suh, 1997; Duncan, Spence, and Mummery, 2005; Seligman, 2002), happiness (Inglehart and Klingemann, 2000; Smith, 2004), and quality of life (which is more often used in the context of patients; Molassiotis et al., 2011; Zatzick et al., 2014). The Behavioral Risk Factor Surveillance System (BRFSS) conducted by CDC includes some items related to well-being, such as questions on life satisfaction (e.g., "In general, how satisfied are you with your life?") and health-related quality of life (e.g., "Would you say that in general your health is excellent/very good/good/fair/poor?") (BRFSS, 2013). The U.S. National Academy of Sciences has established a panel called Measuring Subjective Well-Being in a Policy Relevant Framework, which provides a template for how subjective data can be leveraged with these other data. Well-being research also focuses on certain key populations that are subject to high levels of stress. Examples include caregivers (Gitlin et al., 2003; Pinquart and Sörensen, 2003; Raina et al., 2005), the unemployed (Flatau, Galea, and Petridis, 2000; Friedland and Price, 2003; Warr, 1988), and neglected children (into adulthood) (Buehler et al., 2000; Carpenter and Clyman, 2004; National Survey of Child and Adolescent Well-Being, 2013; Whetten et al., 2009). Research has revealed disparities in well-being by race, sex, and income, which is particularly problematic because healthy individuals tend to be happy individuals and healthy communities tend to be happy communities (Subramanian, Kim, and Kawachi, 2005). Measuring well-being, and understanding the drivers of well-being and how they interact, can help create a more holistic and informed policymaking approach (Kahneman et al., 2004). Moreover, well-being measures can provide important insight for health policymaking beyond economic indicators (Diener and Seligman, 2004).

In terms of operationalizing the concept of community well-being in research, subjective measurement may be the aggregation of responses to questions about individual well-being up to the community level. However, objective community well-being measurement domains include community health, resilience, resource access, educational capacity, and environmental adaptation (Chandra et al., 2013; Wiseman and Brasher, 2008; World Health Organization, 2005). In urban settings, community well-being is nested in an extensive body of neighborhood research (Diez Roux and Mair, 2010; Galea and Vlahov, 2005; Sampson, 2003). It is also 
important to recognize and address differences in individual well-being over the life span and across communities and cultures (Deaton, 2007; United Nations Development Programme, 2014). It should therefore be important to consider how community well-being differs by context. For instance, life satisfaction declines with age more in poorer countries than in richer countries (Deaton, 2007).

\section{Managed Chronic Disease and Reduced Toxic Stress}

Chronic disease is the leading cause of mortality in the world, and as the population ages, the complexity of those chronic conditions only magnifies (Yach et al., 2004). Approximately half of all Americans suffer from one or more chronic diseases (Ward et al., 2014). In 2020, the number of people with chronic conditions is expected to grow to 157 million. By 2023, the number of people with chronic mental disorders is projected to increase from 30 million to 47 million (Ward et al., 2014). In addition, there are significant disparities, with the burden of chronic conditions disproportionately experienced by low-income people and ethnic minorities (Bodenheimer, Chen, and Bennett, 2009). The human and economic toll of chronic disease on society is significant, including lost productivity (DeVol et al., 2007), reduced quality of life (Alonso et al., 2004; Saarni et al., 2006; Sprangers et al., 2000), and increased health care costs (Schneider, O'Donnell, and Dean, 2009; Tinetti, Fried, and Boyd, 2012). The Culture of Health action framework focuses on reducing preventable disease but also on improving the care and management of existing disease, knowing that many are burdened. Observed improvement in chronic disease burden will likely occur through multiple mechanisms, including health care access, primary care, health education, and improvements in self-efficacy, all of which are integrated into the drivers noted in the action framework.

In addition to estimating the prevalence and burden of adult chronic disease, more research has focused on the life span production of disease and, in particular, the relationship between the toxic stress of childhood trauma (e.g., abuse, neglect; see Center on the Developing Child, 2010, 2016a, 2016b; National Scientific Council on the Developing Child, 2010, 2012) and the risk for physical and mental illness in adulthood. A study of BRFSS data in five states found that a sizeable 59 percent of respondents reported one or more adverse child experiences (ACEs) (Centers for Disease Control and Prevention, 2010). The Adverse Child Experiences study led by CDC and Kaiser Permanente (CDC, 2014b) identified a strong, graded relationship between the 
level of traumatic stress in childhood and poor physical, mental, and behavioral outcomes later in life (e.g., depression, liver disease) (Felitti et al., 1998). Almost two-thirds of the study participants reported at least one ACE, and more than one of five reported three or more ACEs. Given the significant and potentially lasting influence of ACEs on adult diseases over the life span, reducing these events during childhood is an important goal to achieve.

\section{Reduced Health Care Costs}

Rising U.S. health care costs affect virtually everyone because costs are borne by employers, governments, and individuals alike. As such, progress on this driver will require improving efficiency in care delivery, changing payment models (noted in more detail in Chapter Eight), and fostering an individual-level impetus to improve health behaviors. Although the growth in health care spending (rate of change) has slowed in recent years, it is unclear how much of this is due to changing economic conditions (recession) or to structural changes in the health care marketplace as a result of the ACA. Early evidence, however, suggests that changes in the delivery of care, for example, that focus on prevention and better care coordination may result in significant improvements in population health if those changes are able to reduce duplication of services and prevent avoidable hospitalizations. Some of these changes, however, may take time to yield cost savings. More broadly, policies and programs that encourage improvements in health behaviors at the individual level have the potential to yield significant long-term savings, including, for example, reducing tobacco use and obesity, both of which are linked to circulatory conditions that the United States spends nearly \$234 billion per year treating. Thus, reducing U.S. health care costs will require both improvements in our health care delivery system - some of which the ACA and other policies are aiming to do - and changes in individual health behaviors. As such, progress in the four action areas should result in reductions in unnecessary health care costs.

\section{Measures}

The following measures were identified as capturing key aspects of each outcome area. We describe each measure in further detail below and then summarize them in Table 9.1. 


\section{Enhanced Individual and Community Well-Being Measures}

Well-being rating in three areas: health, life satisfaction, and work-life balance. This measure focuses on three components of the OECD Better Life Index (BLI). Part of the mission of the BLI is to develop statistics to capture aspects of life that contribute to the quality of people's lives. OECD has identified 11 action areas as being essential to well-being: housing, income, jobs, community, education, environment, civic engagement, health, life satisfaction, safety, and work-life balance. OECD relied on best practices to build composite indicators from multiple sources of underlying data. For instance, the health component is based on the OECD Health Database, life satisfaction is based on the Gallup World Poll, and work-life balance is based on the American Time Use Survey. While the BLI is updated annually, the underlying data sources are not necessarily from the current or previous year. According to OECD, comparisons over time should not be made, given that the methodology is being revised and that the indicators may not change enough to show meaningful movement. The BLI is, however, available across multiple countries, which provides information on social inequalities, as well as useful comparisons as benchmarks of progress toward a Culture of Health in the United States. Wellbeing rating in the three OECD BLI areas selected for monitoring the Culture of Health action framework focus on indicators that capture whether populations are flourishing and the relationship of flourishing to health. Given the interest in capturing well-being outcomes and the interest in flourishing specifically as part of that well-being development, these three measures (health, life satisfaction, work-life balance) were chosen within the BLI. Furthermore, these indicators were considered to have population-level data that would be more responsive to progress in achieving a Culture of Health, in comparison to more static indicators, such as educational attainment or air pollution.

Average amount of out-of-pocket spending on caregiving; impact on caregiver financial and emotional health. Caregivers fill an essential need for many people in need of assistance, including the elderly, the chronically ill, children with special needs, and injured or wounded military personnel and veterans. Caregiving enables the recipients to live better-quality lives and can result in faster and better rehabilitation and recovery. Yet playing this role can impose a substantial physical, emotional, and financial toll on caregivers, potentially resulting in indirect health effects on the caregivers themselves and lower-quality care for the recipients. Using data from the 2008 panel of the National Health and Retirement Survey (HRS), the MetLife Study of 
Caregiving Costs to Working Caregivers (MetLife, 2011) has shown the following: "Adult children 50+ who work and provide care to a parent are more likely to have fair or poor health than those who do not provide care to their parents." Moreover, the impact of caregiving may also be disproportionate for those with fewer other resources. For instance, the MetLife study (MetLife, 2011) indicated that a larger amount of lost wages due to caregiving occurs for women than for men.

The caregiving burden may fall disproportionately on different population groups for several possible reasons. Informal caregiving may be more likely if formal caregiving is unaffordable. Some groups or communities may also prefer or call for caregiving (rather than formal health care) based on cultural expectations. Measuring the amount of burden or commitment for these caregivers can illustrate how much informal health care these caregivers shoulder as chronic disease patterns change in the United States. Policies and programs can reduce the cost of caregiving, but that research is evolving. For example, for seniors, the presence of senior villages or other age-friendly initiatives may reduce the burden on caregivers while improving health outcomes.

\section{Managed Chronic Disease and Reduced Toxic Stress Measures}

\section{Percentage of parents or guardians reporting that their child had two or more family-related} ACEs. Early life experiences that are stressful or traumatic can have continuing health consequences over the life span. ACEs include a broad range of traumatic or stressful experiences that can induce a toxic stress response, and, thus, they encompass a number of potential factors or drivers. ACEs are associated with premature mortality (Brown et al., 2009) and serious health conditions (e.g., ischemic heart disease, stroke, cancer, diabetes; Center for Youth Wellness, 2014). In addition, ACEs are associated with higher allostatic load (i.e., wear and tear on the body) for both men and women after adjustment for early life factors and childhood pathologies. Early psychosocial stress has an indirect but lasting impact on physiological wear and tear via health behaviors, BMI, and socioeconomic factors in adulthood (Solís-Vivanco et al., 2014). Individuals reporting low levels of love and affection and high levels of abuse in childhood report the highest multisystem risk in adulthood (Taylor, Doane, and Eisenberg, 2013). A number of studies show associations between ACEs and neurological conditions (Mehta and Partin, 2007; Teicher, Anderson, and Polcari, 2012). Measuring ACEs for 
children (as opposed to adults) allows us to target interventions and allocate mental health resources to children to improve the likelihood of a successful adulthood.

For this measure, we use a modified version of nine adverse childhood experiences: (1) socioeconomic hardship, (2) divorce/separation of parent, (3) death of parent, (4) parent served time in jail, (5) witness to domestic violence, (6) victim of neighborhood violence, (7) lived with someone who was mentally ill or suicidal, (8) lived with someone with alcohol/drug problem, and (9) was treated or judged unfairly due to race/ethnicity. A response of "somewhat often" or "very often" is coded as an adverse family experience.

There are a number of recommended interventions to prevent adverse childhood experiences and the trauma that may result. WHO recommends interventions to prevent child abuse and its mental health consequences, such as home visits, parent education, and school-based programs. These recommendations are based on evidence regarding the impact of these interventions on direct outcome measures, as well as risk factors (World Health Organization, 2006). The Institute of Medicine also describes similar, evidence-based components of successful interventions to prevent child abuse and neglect (Institute of Medicine, 2013).

Number of disability-adjusted life years (DALYS) of the top ten U.S. chronic diseases. DALYs are a universal metric that allows researchers to broadly compare health across multiple chronic diseases and potentially help decisionmakers and researchers to target funding and interventions (Institute for Health Metrics and Evaluation [IHME], 2016). This metric, first reported as part of the Global Burden of Diseases, Injuries, and Risk Factors Study 2010 (Feigin et al., 2014), stems from a worldwide project that employed cross-discipline researchers and techniques to produce comprehensive estimates and publicly available data visualization tools. IHME provides a list of published evidence using global burden of disease as an outcome for various health interventions (see IHME, 2016).

To compare burdens of disease, calculations of DALYs consider the age at death and the life expectancy of persons affected by each disease and take into account the degree of disability (e.g., discomfort, pain, or functional limitations) imposed by each condition on those who live with the disease. The selected measure describes disease burden from the ten chronic diseases that have the most serious consequences or impacts on the U.S. population in 2010. Understanding and projecting how the most onerous diseases affect people's lives can help 
determine where to make investments or conduct research that will have the greatest benefit to quality of life.

Average health care expenditure by family. Affordable and high-quality health care fosters positive child development, a healthy environment, and livelihoods that are not significantly jeopardized by health care costs. As its health care spending burden increases, a family has fewer available resources to devote to healthy behaviors.

This measure is intended to capture how increases in health care costs affect the finances of a typical American family. It combines data from multiple sources to depict the effects of rising health care costs on a median-income married couple with two children covered by employersponsored insurance (Auerbach and Kellermann, 2011). The measure contains four components: employee premium contribution, employer premium contribution (a hidden cost that indirectly reduces wages and, thus, family income), out-of-pocket spending, and taxes devoted to health care. Using this measure, Auerbach and Kellerman (2011) showed increases in family health care costs over time, comparing this growth to changes in income gains over the same period.

This measure is unique in describing health costs in terms of the household or family unit, as opposed to most measures that focus either on individuals or larger population groups. Further, this measure captures the impact of several types of health care costs, as opposed to others (e.g., the Milliman Medical Index) that inventory only insurance-related costs. The measure is calculated using several assumptions, such as using the same out-of-pocket costs for all individuals younger than 65 , even though out-of-pocket costs for children may be much lower, and using the same tax rate across geographic regions.

Overall U.S. hospitalization rates for chronic and acute conditions per 100,000 population, (potentially preventable hospitalization rates). Potentially preventable hospitalizations are hospital visits that could be avoided with better access to high-quality outpatient care, such as care provided in doctors' offices, clinics, or other settings outside the hospital. This measure includes rates of such hospitalizations related to the acute conditions of urinary infections, pneumonia, and dehydration and the chronic conditions of diabetes, congestive heart failure, hypertension, angina, chronic obstructive pulmonary disease, and asthma.

This measure can be used with hospital inpatient discharge data to describe the quality of health care for ambulatory care-sensitive conditions. These are conditions for which good 
outpatient care can potentially prevent the need for hospitalization or for which early intervention can prevent complications or more severe disease.

A study from AHRQ (Davies et al., 2009) evaluated the use of individual indicators but not of the composite measures. In this report, several interventions are listed that could reduce admission rates by area, provider, and payer. In addition to tracking quality of outpatient care, this measure can be considered a proxy for health (specifically, well-managed illness) among patients with acute and chronic conditions. Interventions targeting improved outpatient care might move the needle on these measures, which would be treated as outcomes.

This measure is particularly useful as a measure of health that focuses on individuals living with chronic disease. However, these measures are typically used for benchmarking hospital quality of care for ambulatory care-sensitive conditions and not explicitly intended as a national measure for health. Using the measure in this way is therefore novel, but further investigation of the primary factors influencing diversion away from hospitalization (e.g., better preventive care versus more lifestyle changes in diet and exercise) is still needed.

Annual average Medicare payment per decedent in the last year of life. Most people who die in America are elderly Medicare beneficiaries, and the program is grappling with its long-term financial viability. For Medicare, spending on care at the end of life is far greater than its spending on a typical beneficiary. The Centers for Medicare \& Medicaid Services estimate that over 25 percent of Medicare spending goes toward the 5 percent of beneficiaries who die each year (CMS, n.d.).

End-of-life care continues to be characterized by aggressive medical intervention and extremely high costs. In 1995, the Study to Understand Prognosis and Preferences for Outcomes and Risks of Treatment (SUPPORT) (Connors et al., 1995) found a pattern of end-of-life treatment decisions not based on timely discussion of the goals of care. Persons in their last year of life require Medicare payments that are six times greater than the cost for a survivor. In 2006, Medicare spent an average of $\$ 38,975$ per decedent, compared to $\$ 5,993$ for other beneficiaries (Riley and Lubitz, 2010). Teno et al. (2013) found that among Medicare beneficiaries who died in 2009 and 2005, compared with those who died in 2000, a lower proportion died in an acute care hospital, although both intensive care unit use and the rate of health care transitions increased in the last month of life. 
There is a great deal of room for improvement on closing the gap between people's wishes for how and where to die and where people actually end up dying. Earlier research (e.g., SUPPORT) indicates a disconnect between decisions on end-of-life care and previous discussions of care goals. However, between 1989 and 2007, there was a shift in the places where Americans die, with more people dying at home and fewer dying in institutional settings (Heath, Romero, and Reynolds, 2013). In addition, race and ethnicity have been identified as factors that affect end-of-life care and place of death. A much higher proportion of whites are dying at home or in a nursing home compared to all other races (Heath, Romero, and Reynolds, 2013).

Shifting end-of-life care away from hospitals and toward home or hospice may result in wellbeing benefits in the last year of life. This measure describes expenditures associated with annual end-of-life care using Medicare claims data. This measure only characterizes the cost of care for people in the last year of life but does not yet capture the quality of end-of-life care. The measure will evolve as the Culture of Health initiative progresses.

Table 9.1 summarizes the measures that have been identified for the outcome areas of the action framework. These measures demonstrate the underlying concepts of each outcome area.

Table 9.1. Population Health, Well-Being, and Equity: Measures by Outcome Area

\begin{tabular}{|l|l|l|}
\hline \multicolumn{1}{|c|}{ Outcome Area } & \multicolumn{1}{|c|}{ Measure } & \multicolumn{1}{|c|}{$\begin{array}{c}\text { What It Means for Outcomes/ } \\
\text { Culture of Health Overall }\end{array}$} \\
\hline $\begin{array}{l}\text { Enhanced individual and } \\
\text { community well-being }\end{array}$ & $\begin{array}{l}\text { Well-being rating on three sub- } \\
\text { action areas: health, life } \\
\text { satisfaction, work-life balance }\end{array}$ & $\begin{array}{l}\text { Indicates how much communities and } \\
\text { individuals are thriving and flourishing, a } \\
\text { key set of indicators for well-being }\end{array}$ \\
\hline $\begin{array}{l}\text { Enhanced individual and } \\
\text { community well-being }\end{array}$ & $\begin{array}{l}\text { Caregiving burden: Average } \\
\text { amount of out-of-pocket spending } \\
\text { on caregiving; (additional } \\
\text { measure) impact on caregiver } \\
\text { financial and emotional health }\end{array}$ & $\begin{array}{l}\text { Includes a substantial proportion of the } \\
\text { population that is not routinely considered } \\
\text { in health research. The well-being of } \\
\text { nonprofessional care providers is often } \\
\text { overlooked but also has implications for the } \\
\text { overall health and well-being of those for } \\
\text { whom they are caring }\end{array}$ \\
\hline $\begin{array}{l}\text { Managed chronic } \\
\text { disease and reduced } \\
\text { toxic stress }\end{array}$ & $\begin{array}{l}\text { Adverse childhood experiences: } \\
\text { Percentage of parents or guardians } \\
\text { reporting that their child had two } \\
\text { or more family-related ACEs }\end{array}$ & $\begin{array}{l}\text { Provides information about events during a } \\
\text { key period of life that may influence people } \\
\text { over their entire life span and increase the } \\
\text { chance of chronic disease and poor well- } \\
\text { being }\end{array}$ \\
\hline
\end{tabular}




\begin{tabular}{|l|l|l|}
\hline \multicolumn{1}{|c|}{ Outcome Area } & \multicolumn{1}{|c|}{ Measure } & \multicolumn{1}{|c|}{$\begin{array}{c}\text { What It Means for Outcomes/ } \\
\text { Culture of Health Overall }\end{array}$} \\
\hline $\begin{array}{l}\text { Managed chronic } \\
\text { disease and reduced } \\
\text { toxic stress }\end{array}$ & $\begin{array}{l}\text { Number of disability-adjusted life } \\
\text { years (DALYs) of the top ten } \\
\text { chronic diseases in the country }\end{array}$ & $\begin{array}{l}\text { Indicates burden of chronic disease (asthma, } \\
\text { cancer, chronic obstructive pulmonary } \\
\text { disease, cardiovascular disease, diabetes, } \\
\text { other) and the related quality of life }\end{array}$ \\
\hline $\begin{array}{l}\text { Reduced health care } \\
\text { costs }\end{array}$ & $\begin{array}{l}\text { Average health care expenditure } \\
\text { by family }\end{array}$ & $\begin{array}{l}\text { Offers a broader picture of how much } \\
\text { families spend on health, which should } \\
\text { reduce if progress is made }\end{array}$ \\
\hline $\begin{array}{l}\text { Reduced health care } \\
\text { costs }\end{array}$ & $\begin{array}{l}\text { Overall U.S. hospitalization rates } \\
\text { for potentially preventable chronic } \\
\text { and acute conditions per 100,000 } \\
\text { population }\end{array}$ & $\begin{array}{l}\text { Serves as a multilevel indicator of } \\
\text { improvements in health care, including } \\
\text { primary care, self-care, health education, } \\
\text { and quality of life }\end{array}$ \\
\hline $\begin{array}{l}\text { Reduced health care } \\
\text { costs }\end{array}$ & $\begin{array}{l}\text { End-of-life expenditures: annual } \\
\text { average Medicare payment per } \\
\text { decedent in the last year of life }\end{array}$ & $\begin{array}{l}\text { Tracks changes in the way people are dying } \\
\text { (e.g., inside versus outside a medical } \\
\text { setting, growth in end-of-life planning); a } \\
\text { wellness indicator }\end{array}$ \\
\hline
\end{tabular}

\section{Limitations}

While the measures described above represent some options in each outcome area, it is important to note a few measurement challenges in the outcomes action area.

There is very little systematic collection of data on well-being. While communities are starting to track such well-being indexes as sense of community, flourishing, and opportunity, this is not nearly common practice. As such, we are limited in the sources of survey or administrative data that capture core well-being concepts. The Culture of Health effort should provide an opportunity to advance that discussion and encourage those conducting national and local data collection efforts to incorporate more of these well-being measures. Taking advantage of quantifiable, naturalistic data sources, such as social media, mobile devices, or other digital outputs, can help move the measurement of well-being beyond reliance on self-reported information. For instance, social media (e.g., Twitter) data may be used to estimate populationlevel measures that include life satisfaction (Schwartz et al., 2014), happiness (Mitchell et al., 2013), and heart disease mortality (Eichstaedt et al., 2015).

Not all the outcomes in the framework are collected routinely. For example, BRFSS used to collect information about ACEs among adults, but it stopped asking about ACEs in 2012. The Youth Behavioral Risk Survey collects only a subset of ACE-related questions, mainly about risky behaviors. Our data source, the National Survey of Children's Health, is fielded every four 
years and will not capture accumulation of ACEs in the same panel or with the nuance of developmental transitions in ACE experience or exposure.

\section{Chronic disease analyses require more investigation of social network and community}

impacts. While Murray et al. (2012) and other researchers have advanced our understanding of the global burden of chronic disease, we are still missing analyses to demonstrate the impact of disease on the wider social network of an individual (e.g., caregiver burden) or social impacts in the broader community. These data are critical if we are able to advance national dialogue about the interdependency of health and the value of community-level health investments. To date, these data are difficult to capture across all communities. Further, if we include adverse childhood experiences and toxic stress among other childhood stressors that predict disease burden later in life, we need far more systematic use of these measures across nationally representative surveys. 


\section{Summary and Next Steps}

This report provides foundational content for a vision of a Culture of Health, as well as the four action areas of the Culture of Health action framework. The report offers background to outline the conceptual underpinnings of the four action areas, the outcome area, how each is defined, the drivers in each action area, and the measures. In this final chapter, we briefly summarize key themes from this analysis of action areas and current measurement gaps that cut across action areas. Then we provide an overview of the next steps in implementation of this action framework.

\section{Current Measurement Gaps}

The initial efforts to define and frame each of the action areas to advance the ten principles of a Culture of Health illustrated opportunities to achieve that end, as well as gaps in current measurement. Given the groundbreaking nature of RWJF's initiative, the team anticipated that there would be limitations in the availability of measures that track progress in such areas as health promotion, community capacity for health, cross-sectoral collaboration, shared values, and well-being development. The environmental scan and first phase of stakeholder engagement underscore this challenge. While each chapter provides more detailed description of challenges in measurement within action area, we briefly summarize themes that cut across action areas.

There are limitations in measures that represent Culture of Health capacity. In some areas, such as health care system access and quality, there are measures that capture the performance of actors and/or the system. In other action areas, such as multi-sector collaboration, there are measures that document progress toward achieving collaboration, including the number and, in some cases, the nature of partnerships. However, to determine national and community ability to promote a Culture of Health, we also need measures that assess the capacity of people, organizations, and systems to consistently build community and conduct activities that will promote policies and environments for health and well-being. A Culture of Health affords a unique opportunity to strengthen these capacity measures. For example, we could craft a measure that assesses community readiness to adopt health-promoting policies or community conditions to support health-promoting programs, but those measures do not exist widely. 
There are few measures that describe or assess the decision environment or the dynamic nature of Culture of Health development. In Chapter One, we outlined the characteristics of culture as bounded, aligned, shared, and dynamic. Further, we briefly discussed the interaction between individuals and organizations to support decisions that advance health. Yet, our current analysis did not yield many measures that monitor or evaluate this decision environment or the conditions for change. In addition, if cultural change requires alignment within and across sectors on behalf of health, we do not have robust or tested measures in this space. These measures may need to be constructed based on a few other measures (e.g., linking policy adoption with community or social characteristics). Even when measures can be developed, the availability of national data may be a challenge.

We have not yet identified measures that acknowledge change in the Culture of Health over time or across varying context. The Culture of Health and its measurement strategy are premised on the fact that cultural shifts take time. Further, there are multiple cultures of health that can be influenced by varying emphases on drivers within each action area. RWJF is dedicated to supporting a national dialogue over the short term and the long term (i.e., a generation, or 20 years). With that interest and investment, it will be important not to rely on static measures for a Culture of Health. For each action area in the action framework, the drivers will hold constant, but the actual measures can evolve over time as improvements or advancements in the Culture of Health are made and societal conditions change. It will be important to develop a flexible measurement model or framework that allows for progressive metrics, which can be adaptive to both time and diverse, local contexts.

\section{Next Steps with Action Framework}

This report was intended to summarize the first developmental phase and evidence base guiding the Culture of Health action framework (Figure 1.2). The report is supplemented with other materials, including more detail on measures and the final RWJF report, From Vision to Action: A Framework and Measures to Mobilize a Culture of Health (Plough et al., 2015). For more information on frequently asked questions related to the action framework, see Appendix A.

Over the next phase of the project, sentinel communities will complement national monitoring of the Culture of Health, described in the next section. We also describe 
considerations in implementation as the framework is used and as illustrative measures continue to be tested.

\section{Sentinel Communities}

The sentinel community phase of this project started in late 2015 and will be critical to understanding how communities are developing (or not developing) a Culture of Health, what data they use to track that development, and how certain Culture of Health measure data could be collected and analyzed as the national effort moves forward (see Appendix B). This design builds on WHO's model of sentinel surveillance to explore development of a Culture of Health through deliberate selection of sites, rather than through passive or population-based approaches. Sentinel design allows monitoring of the emergence or progression of a cultural shift. RTI International is working with RWJF and RAND on sentinel community data collection (see www.cultureofhealth.org for site list).

Sentinel communities allow the team to complement national Culture of Health measures with more granularity on cultural change processes (or barriers), cross-sectoral engagement, and individual and community efforts to change the context in which health policies are developed and healthier conditions are created. The hope is to identify drivers of change and likely signals that allow assessment of how communities move toward culture. The sentinel communities are not demonstration or place-based communities, but rather sites for naturalistically testing local Culture of Health measurement and overall progress. Objectives of sentinel communities are as follows:

- to determine how communities are developing a Culture of Health within and across the RWJF action framework action areas; what factors facilitate or impede the development of a Culture of Health; and what motivations, collaborations, and systems are key to supporting a Culture of Health

- to provide a rich and detailed narrative of community context and change through the collection of quantitative and qualitative data on each community

- to identify opportunities for additional measure development around community innovation.

Communities have been selected based on variation by demographic characteristics (population size and composition by income, race/ethnicity, age, other), geographic distribution 
(rural/urban, location in the United States), and community readiness for culture of change based on such factors as

- participation in community health initiatives

- political or social readiness, or other related factors

- engagement of sectors

- other community action policies (e.g., adoption of specific health policies).

Questions to be addressed in sentinel communities include the following:

1. What are the factors that influence development of a Culture of Health, and how do those vary by demographic, social system, and other characteristics?

2. What do communities employ now to develop a Culture of Health, how do they implement/expand their efforts for a Culture of Health, and in what ways?

3. How do communities sustain Culture of Health practices and policies and what factors support that maintenance, integration into routine functioning, and so on?

4. How do communities move along the Culture of Health continuum, and where do they get stuck in the process (within and across action areas)?

5. How do communities track progress on a Culture of Health with existing data? How might communities use the Culture of Health measures to help them track progress, as well as catalyze change?

6. How are communities learning from other communities? How are they sharing best practices?

\section{Implementation: Next Steps}

The team (RWJF and RAND) will work to evolve the measures and build out the larger measurement strategy for the action framework. Measurement strategy includes guidance on how measures are tested and validated (e.g., processes for measures prototyping), instructions on how measures may apply to national and/or local context (e.g., factors to consider in local use or consumption), and insights for how measures are framed and communicated for wider, national consumption (e.g., core audience, key messages). This measurement strategy will need to acknowledge structural variables that support measures implementation and long-term use. This includes factors related to sectoral roles and engagement, governance structure and alignment to local processes, and coordination and integration with shared accountability structures nationally 
and locally (Kickbusch and Gleicher, 2012). Further, the development of the measurement strategy for a Culture of Health also offers RWJF an opportunity to coordinate this discussion with other national conversations on complementary strategy in resilience, sustainability, and civic well-being measurement. These elements of the measurement strategy will be explored further in subsequent phases of the project.

This effort represents an attempt to define and disseminate measures in a new and emerging area in which the knowledge base, data sources, and technologies for accessing data are changing rapidly. The measures are intended not just as "thermometers" of change, but as catalysts or tools that engage and support communities in improving their Culture of Health. As such, the ultimate impact of the measures is not a foregone conclusion but depends considerably on how individuals and organizations use them in specific community contexts. In rapidly changing, context-sensitive enterprises such as this, an implementation plan should not be a script to be followed to the letter, but a framework for learning and adaption. This section sets out such a learning/adaptation framework to guide implementation and evolution of the Culture of Health measures in the coming years.

We anticipate several occurrences that might prompt changes and evolution in the measures. Each, in turn, defines a learning objective that must be addressed in the implementation plan:

1. Measures are "topped out," and additional improvement is not possible. In such situations, RWJF and its partners will need to identify metrics that represent later stages in the change process - e.g., from a measure focusing on the existence of a plan to a measure of its actual implementation or a measure of its quality.

2. New knowledge becomes available. For instance, new knowledge might emerge about the observable attributes of a Culture of Health or about the factors (e.g., investments, policies) that support or hinder its development and spread. In such instances, RWJF and its partners will need to find measures of these new attributes and factors.

3. New data sources become available. For instance, new questions addressing Culture of Health topics may become available, or new data sources and technologies for accessing and analyzing data (e.g., big data) on Culture of Health constructs may become available.

4. Improved understanding of how individuals and organizations use Culture of Health measures. Because Culture of Health measures are intended as catalysts, knowledge of 
how people use them is critical. Given the large number of sectors, partners, and communities involved, it is difficult to anticipate this up front.

With that in mind, the first measures initiate a continuous learning loop summarized in Figure 10.1. It includes (1) an ongoing review of evidence and data sources, (2) learning from use contexts, (3) synthesis of findings and identifying the need for new or revised measures, and (4) making updates to the Culture of Health measure set. The implementation cycle takes approximately one year, after which it repeats.

Figure 10.1. Learning Process for Culture of Health Measures

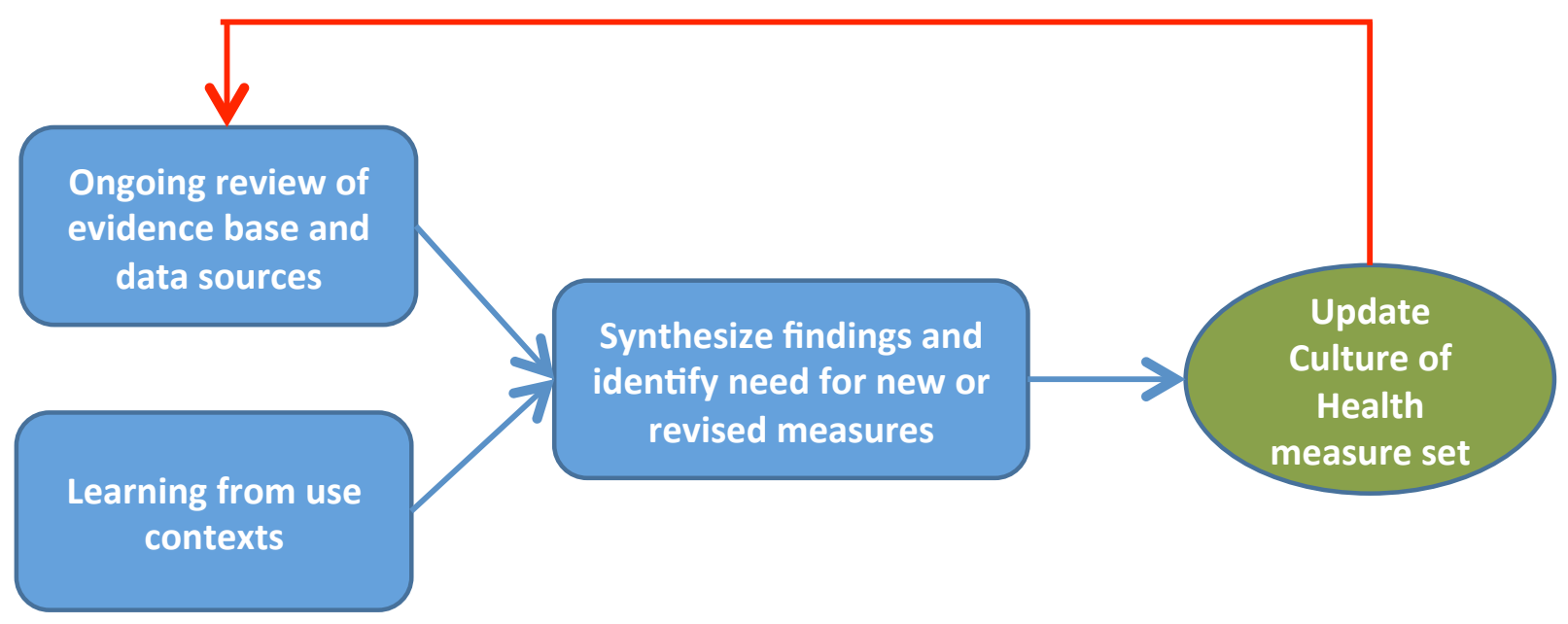

Continuous Review of Evidence and Data Sources

RWJF will work with key partners to stay abreast of important developments in the research literature on the Culture of Health and on relevant data sources. To facilitate this, RWJF will engage with subject matter experts and experts familiar with the literature on a given field; available data sources; and relevant approaches to analyzing, visualizing, and understanding the data used by the Culture of Health measures. It will ask the experts to comment on the measures, applying the measure criteria used to select the Culture of Health measures (described earlier in this report). Special attention will be paid to measures that are "topping out" - that is, reaching a point where additional improvements could not be captured — or are close to doing so. 


\section{Learning from Use Contexts}

The RWJF team with RAND will closely monitor findings from the sentinel communities and, as needed, commission special analyses or data collection designed to better understand how the Culture of Health metrics are being used by individuals and organizations. Specific questions may include (but are not limited to) the following:

- How do users become aware of Culture of Health metrics? What factors appear to account for variations in awareness?

- What specific activities do the Culture of Health measures inform? Examples might include framing discussions, agenda-setting, and decisionmaking. In some instances, the influence of a measure lies in the specific quantitative feedback it provides (e.g., the number or percentage of communities with a particular amenity), while in other cases the primary impact of the measure lies in capturing attention and stimulating key points of discussion.

- Do individuals and organizations use the v1.0 measures as inspiration for creating or adapting their own measures, or do they use the v1.0 measures "as is"?

- What contextual factors influence use? These might include governance structures, social networks/relationships among trusted partners, and past experience with similar initiatives.

- Are there important groups that are not aware of the measures or are not using them? What are the barriers?

The team will also look for opportunities to develop use case studies and/or simple logic models that illustrate in concrete terms how these various activities and contextual factors operate in conjunction.

\section{Review/Synthesis and Building the Next Version}

In the next year, the team will convene key partners to review key findings from the evidence/data review and the use case analysis. The group will identify a small number of measures that would most benefit from improvement or replacement. In most years, we expect the number of such measures to be relatively small, and the group will seek to balance the benefits of improving/replacing measures against the competing benefits of tracking the same 
measures over time. We expect a refreshed (or v2.0) list to be available in 2017, though the majority of measures will not change. 


\section{References}

Abbasi, M.-A., Chai, S.-K., Liu, H., \& Sagoo, K. (2012). Real-world behavior analysis through a social media lens. In Social Computing, Behavioral-Cultural Modeling and Prediction (pp. 18-26). Berlin: Springer.

Acevedo-Garcia, D., \& Lochner, K. A. (2003). Residential segregation and health. In I. Kawachi \& L. Berkman (eds.), Neighborhoods and Health (pp. 265-287). New York: Oxford University Press.

Acevedo-Garcia, D., Lochner, K. A., Osypuk, T. L., \& Subramanian, S. V. (2003). Future directions in residential segregation and health research: A multilevel approach. American Journal of Public Health, 93(2), 215-221. doi: 10.2105/AJPH.93.2.215

Acevedo-Garcia, D., Osypuk, T. L., Werbel, R. E., Meara, E. R., Cutler, D. M., \& Berkman, L. F. (2004). Does housing mobility policy improve health? Housing Policy Debate, 15(1), 49-98.

Acosta, J. D., Whitley, M. D., May, L. W., Dubowitz, T., Williams, M. V., \& Chandra, A. (2015). Stakeholder perspectives on a culture of health: Key findings (RR-1274-RWJ). Santa Monica, CA: RAND Corporation. As of February 25, 2016: http://www.rand.org/pubs/research_reports/RR1274.html

Aday, L. A., \& Andersen, R. (1974). A framework for the study of access to medical care. Health Services Research, 9(3), 208-220.

Adler, N. E., \& Newman, K. (2002). Socioeconomic disparities in health: Pathways and policies. Health Affairs, 21(2), 60-76.

Agency for Healthcare Research and Quality. (2013). Module 16: Introduction to the Care Model. Rockville, MD.

Ajzen, I., \& Timko, C. (1986). Correspondence between health attitudes and behavior. Basic and Applied Social Psychology 7(4), 259-276.

Alonso, J., Ferrer, M., Gandek, B., Ware Jr, J. E., Aaronson, N. K., Mosconi, P., . . Kaasa, S. (2004). Health-related quality of life associated with chronic conditions in eight countries: Results from the International Quality of Life Assessment (IQOLA) Project. Quality of Life Research, 13(2), 283-298. doi: 10.1023/B:QURE.0000018472.46236.05

American Hospital Association. (2013). Engaging health care users: A framework for healthy individuals and communities. Chicago: American Hospital Association.

American Library Association. (2014). Affordable Care Act (ACA). As of January 18, 2016 http://www.ala.org/tools/atoz/affordable-care-act

American Public Health Association. (2009). Support for community health workers to increase health access and to reduce health inequities: Policy number: 20091. As of January 20, 2016:

https://www.apha.org/policies-and-advocacy/public-health-policy-statements/ policy-database/2014/07/09/14/19/support-for-community-health-workers-to-increasehealth-access-and-to-reduce-health-inequities

American Telemedicine Association. (2015). Research outcomes: Telemedicine's impact on healthcase cost and quality. Washington, DC. 
An, R., Patel, D., Segal, D., \& Sturm, R. (2013). Eating better for less: A national discount program for healthy food purchases in South Africa. American Journal of Health Behavior, 37(1), 56-61.

Anderson, L. M., Shinn, C., St, C. J., Fullilove, M. T., Scrimshaw, S. C., Fielding, J. E., . . Richardson, T. (2002). Community interventions to promote healthy social environments: Early childhood development and family housing. A report on recommendations of the Task Force on Community Preventive Services. MMWR, 51(RR-1), 1-8.

Andreyeva, T., Long, M. W., \& Brownell, K. D. (2010). The impact of food prices on consumption: A systematic review of research on the price elasticity of demand for food. American Journal of Public Health, 100(2), 216-222.

Anheier, H. (2004). Civil society: Measurement, evaluation, policy. Sterling, VA: Earthscan.

Antonelli, R. C., McAllister, J. W., \& Popp, J. (2009). Making care coordination a critical component of the pediatric health system: A multidisciplinary framework. New York: Commonwealth Fund.

Arnstein, P., Vidal, M., Wells-Federman, C., Morgan, B., \& Caudill, M. (2002). From chronic pain patient to peer: Benefits and risks of volunteering. Pain Management Nursing, 3(3), 94-103.

Artazcoz, L., Benach, J., Borrell, C., \& Cortès, I. (2005). Social inequalities in the impact of flexible employment on different domains of psychosocial health. Journal of Epidemiology and Community Health, 59(9), 761-767.

Assistant Secretary for Planning and Evaluation. (2013, December 11). Health insurance marketplace: December enrollment report for the period: October 1-November 30. As of February 18, 2016: https://aspe.hhs.gov/sites/default/files/pdf/76726/ib_2013dec_enrollment.pdf

Auerbach, D. I., \& Kellermann, A. L. (2011). A decade of health care cost growth has wiped out real income gains for an average US family. Health Affairs (Millwood), 30(9), 16301636. doi: 10.1377/hlthaff.2011.0585

Babey, S. H., Hastert, T. A., \& Brown, E. R. (2007). Teens living in disadvantaged neighborhoods lack access to parks and get less physical activity. Health Policy Research Brief. Los Angeles: UCLA Center for Health Policy Research.

Baezconde-Garbanati, L., Unger, J., Portugal, C., Delgado, J. L., Falcon, A., \& Gaitan, M. (2006). Maximizing participation of Hispanic community-based/non-governmental organizations (NGOs) in emergency preparedness. International Quarterly of Community Health Education, 24(4), 289-317.

Baker, E. L., Melton, R. J., Stange, P. V., Fields, M. L., Koplan, J. P., Guerra, F. A., \& Satcher, D. (1994). Health reform and the health of the public: Forging community health partnerships. JAMA, 272(16), 1276-1282.

Barnett, W. S. (2011). Effectiveness of early educational intervention. Science, 333(6045), 975978. doi: 10.1126/science.1204534

Barr, V., Robinson, S., Marin-Link, B., Underhill, L., Dotts, A., Ravensdale, D., \& Salivaras, S. (2003). The expanded chronic care model. Hospital Quarterly, 7(1), 73-82.

Barrett, L., Plotnikoff, R. C., Raine, K., \& Anderson, D. (2005). Development of measures of organizational leadership for health promotion. Health Education \& Behavior, 32(2), 195-207.

Bauer, U. E., Briss, P. A., Goodman, R. A., \& Bowman, B. A. (2014). Prevention of chronic disease in the 21st century: Elimination of the leading preventable causes of premature 
death and disability in the USA. The Lancet, 384(9937), 45-52. doi: 10.1016/S01406736(14)60648-6

Baumeister, R. F. (1991). Meanings of life. New York: Guilford Press.

Been, J. V., Nurmatov, U. B., Cox, B., Nawrot, T. S., van Schayck, C. P., \& Sheikh, A. (2014). Effect of smoke-free legislation on perinatal and child health: A systematic review and meta-analysis. The Lancet, 383(9928), 1549-1560. doi: 10.1016/S0140-6736(14)60082-9

Behavioral Risk Factor Surveillance System. (2013, December 17). 2014 Behavioral Risk Factor Surveillance System Questionnaire. As of February 18, 2016: http://www.cdc.gov/brfss/questionnaires/pdf-ques/2014_brfss.pdf

Bell, J., Mora, G., Hagan, E., Rubin, V., \& Karpyn, A. (2013). Access to healthy food and why it matters: A review of the research. Philadelphia, PA: The Food Trust.

Bell, J. F., Wilson, J. S., \& Liu, G. C. (2008). Neighborhood greenness and 2-year changes in body mass index of children and youth. American Journal of Preventive Medicine, 35(6), 547-553. doi: 10.1016/j.amepre.2008.07.006

Benedict, R. (1934). Patterns of culture. Boston: Houghton Mifflin Company.

Berenson, R. A., \& Burton, R. A. (2012). Health policy brief: Next steps for ACOs. Health Affairs, 31, 1-6.

Berwick, D. M., Nolan, T. W., \& Whittington, J. (2008). The triple aim: Care, health, and cost. Health Affairs (Millwood), 27(3), 759-769. doi: 10.1377/hlthaff.27.3.759

Bird, S. T., \& Bauman, K. E. (1998). State-level infant, neonatal and postneonatal mortality: The contribution of selected structural socioeconomic variables. International Journal of Health Services, 28(1): 13-27

Bishaw, A. (2014). Changes in areas with concentrated poverty: 2000 to 2010. Washington, DC: United States Census Bureau

Bodenheimer, T., Chen, E., \& Bennett, H. D. (2009). Confronting the growing burden of chronic disease: Can the US health care workforce do the job? Health Affairs, 28(1), 64-74. doi: doi: $10.1377 /$ hlthaff.28.1.64

Boone-Heinonen, J., Casanova, K., Richardson, A. S., \& Gordon-Larsen, P. (2010). Where can they play? Outdoor spaces and physical activity among adolescents in US urbanized areas. Preventive Medicine, 51(3), 295-298.

Bradley, E., \& Taylor, L. (2013). The American health care paradox: Why spending more is getting us less. New York: PublicAffairs.

Bradley, E. H., Elkins, B. R., Herrin, J., \& Elbel, B. (2011). Health and social services expenditures: Associations with health outcomes. BMJ Quality \& Safety, 20, 826-831. doi: doi:10.1136/bmjqs.2010.048363

Brain, D. (2005). From good neighborhoods to sustainable cities: Social science and the social agenda of the new urbanism. International Regional Science Review, 28(2), 217-238.

Braveman, P. (2014). What are health disparities and health equity? We need to be clear. Public Health Reports, 129(Suppl 2), 5-8.

Braveman, P., \& Gruskin, S. (2003). Defining equity in health. Journal of Epidemiology and Community Health, 57(4), 254-258.

BRFSS—see Behavioral Risk Factor Surveillance System.

Brown, D. W., Anda, R. F., Tiemeier, H., Felitti, V. J., Edwards, V. J., Croft, J. B., \& Giles, W. H. (2009). Adverse childhood experiences and the risk of premature mortality. American Journal of Preventive Medicine, 37(5), 389-396. doi: 10.1016/j.amepre.2009.06.021 
Brownson, R. C., Haire-Joshu, D., \& Luke, D. A. (2006). Shaping the context of health: A review of environmental and policy approaches in the prevention of chronic diseases. Annual Review of Public Health, 27, 341-370. doi: 10.1146/annurev.publhealth.27.021405.102137

Buehler, C., Orme, J. G., Post, J., \& Patterson, D. A. (2000). The long-term correlates of family foster care. Children and Youth Services Review, 22(8), 595-625. doi: 10.1016/S01907409(00)00108-0

Built Environment Health Research Group at Columbia University) (n.d.). Neighborhood walkability. As of February 18, 2016: http://beh.columbia.edu/neighborhood-walkability/

Burdick-Will, J. (2013). School violent crime and academic achievement in Chicago. Sociology of education, 86(4). doi: 10.1177/0038040713494225

Carman, K. G., Eibner, C., \& Paddock, S. M. (2015). Trends in health insurance enrollment, 2013-15. Health Affairs, 34(6), 1044-1048. doi: 10.1377/hlthaff.2015.0266

Carpenter, S. C., \& Clyman, R. B. (2004). The long-term emotional and physical wellbeing of women who have lived in kinship care. Children and Youth Services Review, 26(7), 673686. doi: 10.1016/j.childyouth.2004.02.015

Carver, A., Timperio, A., \& Crawford, D. (2008). Playing it safe: The influence of neighbourhood safety on children's physical activity-A review. Health \& Place, 14(2), 217-227.

CDC-see Centers for Disease Control and Prevention.

Center on the Developing Child. (2010). The foundations of lifelong health are built in early childhood. Cambridge, MA: Harvard University. As of February 17, 2016:

http://developingchild.harvard.edu/wp-content/uploads/2010/05/

Foundations-of-Lifelong-Health.pdf

Center on the Developing Child. (2016a). InBrief: The impact of early adversity on children's development. As of February 17, 2016:

http://developingchild.harvard.edu/resources/

inbrief-the-impact-of-early-adversity-on-childrens-development-video

Center on the Developing Child. (2016b). Social and behavioral determinants of toxic stress. As of February 17, 2016:

http://developingchild.harvard.edu/resources/social-behavioral-determinants-toxic-stress/

Center for Youth Wellness. (2014). Homepage. As of January 21, 2016: http://www.centerforyouthwellness.org/

Centers for Disease Control and Prevention. (2007). Homepage. As of January 21, 2016: http://www.cdc.gov/

Centers for Disease Control and Prevention. (2010). Adverse childhood experiences reported by adults: Five states, 2009. MMWR, 59(49), 1609-1613.

Centers for Disease Control and Prevention. (September 2013). Resources for implementing the Community Health Needs Assessment process. As of August 31, 2014: http://www.cdc.gov/policy/chna/

Centers for Disease Control and Prevention \& ASCD. (2014a). Whole School, Whole Community, Whole Child: A collaborative approach to learning and health. Alexandria, VA: ASCD. 
Centers for Disease Control and Prevention. (2014b, May 13). ACE study. As of February 29, 2016:

http://www.cdc.gov/violenceprevention/acestudy/

Centers for Disease Control and Prevention. (2015a, October 14). Teen drivers: Get the facts. As of February 18, 2016: http://www.cdc.gov/motorvehiclesafety/teen_drivers/teendrivers_factsheet.html

Centers for Disease Control and Prevention. (2015b, December 29). Cost data and prevention policies. As of February 18, 2016:

http://www.cdc.gov/motorvehiclesafety/costs/index.html

Centers for Medicare \& Medicaid Services. (n.d.). Last Year of Life Study. As of February 18, 2016:

https://www.cms.gov/Research-Statistics-Data-and-Systems/Research/ActuarialStudies/ downloads/last_year_of_life.pdf

Chandra, A., Lara-Cinisomo, S., Jaycox, L., Tanielian, T. L., Han, B., Burns, R. M., \& Ruder, T. (2011). Views from the homefront: The experiences of youth and spouses from military families (TR-913-NMFA). Santa Monica, CA: RAND Corporation. As of February 25, 2016:

http://www.rand.org/pubs/technical_reports/TR913.html

Chandra, A., Williams, M., Plough, A., Stayton, A., Wells, K. B., Horta, M., \& Tang, J. (2013). Getting actionable about community resilience: The Los Angeles County Community Disaster Resilience project. American Journal of Public Health, 103(7), 1181-1189. doi: 10.2105/AJPH.2013.301270

Chang, V. W., \& Christakis, N. A. (2005). Income inequality and weight status in U.S. metropolitan areas. Social science \& medicine, 61(1), 83-96.

MChavis, D. (2006). Strategic factors for building community: The Five C's: Community, connections, control, cash \& collective action. Baltimore, MD: Campaign Consultation, Inc.

Chida, Y., \& Steptoe, A. (2008). Positive psychological well-being and mortality: A quantitative review of prospective observational studies. Psychosomatic Medicine, 70(7), 741-756. doi: 10.1097/PSY.0b013e31818105ba

Christian, H., Zubrick, S. R., Foster, S., Giles-Corti, B., Bull, F., Wood, L., . . . Boruff, B. (2015). The influence of the neighborhood physical environment on early child health and development: A review and call for research. Health \& Place, 33, 25-36. doi: 10.1016/j.healthplace.2015.01.005

Chu, C., Breucker, G., Harris, N., Stitzel, A., Gan, X., Gu, X., \& Dwyer, S. (2000). Healthpromoting workplaces: International settings development. Health Promotion International, 15(2), 155-167. doi: 10.1093/heapro/15.2.155

Chung, C. (2014, November 4). [Personal communication with author].

Chung, C., \& Pennebaker, J. W. (2007). The psychological functions of function words. In K. Fiedler (Ed.), Social Communication (pp. 343-359). New York: Psychology Press.

City of Santa Monica. (2015). The wellbeing project. As of January 21, 2016: http://wellbeing.smgov.net/

CMS - see Centers for Medicare \& Medicaid Services.

Coaffee, J., \& Healey, P. (2003). "My voice: My place": Tracking transformations in urban governance. Urban Studies, 40(10), 1979-1999.

Cohen, R. (2011). The impacts of affordable housing on health: A research summary. Washington, DC: Center for Housing Policy. 
Cohen, S. (2004). Social relationships and health. American Psychologist, 59(8), 676.

Colby, S. L., \& Ortman, J. M. (2015). Projections of the size and composition of the U.S. population: 2014 to 2060. Report Number: P25-1143. U.S. Census Bureau.

Coleman, E. A., Parry, C., Chalmers, S., \& Min, S.-J. (2006). The care transitions intervention: Results of a randomized controlled trial. Archives of Internal Medicine, 166(17), 18221828. doi: 10.1001/archinte.166.17.1822

Coleman, K., Austin, B. T., Brach, C., \& Wagner, E. H. (2009). Evidence on the Chronic Care Model in the new millennium. Health Affairs, 28(1), 75-85. doi: 10.1377/hlthaff.28.1.75

Collins, S., Rasmussen, P., \& Doty, M. (2014). Gaining ground: Americans' health insurance coverage and access to care after the Affordable Care Act's first open enrollment period. New York: Commonwealth Fund.

The Community Guide. (2015). Promoting health equity through education programs and policies: School-based health centers: Task force and rationale statement. As of January 19, 2016:

http://www.thecommunityguide.org/healthequity/education/

RRschoolbasedhealthcenters.html

Connors, A., Dawson, N., Desbiens, N., et al. (1995, November 22). A controlled trial to improve care for seriously ill hospitalized patients: The study to understand prognoses and preferences for outcomes and risks of treatments (SUPPORT). JAMA. 274(20).

Coombes, E., Jones, A. P., \& Hillsdon, M. (2010). The relationship of physical activity and overweight to objectively measured green space accessibility and use. Social Science \& Medicine, 70(6), 816-822. doi: 10.1016/j.socscimed.2009.11.020

Cornish, F., Montenegro, C., van Reisen, K., Zaka, F., \& Sevitt, J. (2014). Trust the process: Community health psychology after Occupy. Journal of Health Psychology, 19(1), 60-71. doi: $10.1177 / 1359105313500264$

Crompton, T., \& Kasser, T. (2010). Human identity: A missing link in environmental campaigning. Environment, 52(4), 23-33. doi: 10.1080/00139157.2010.493114

Culture of Health. (2016). Home page. As of February 13, 2016: http://www.cultureofhealth.org

Cutter, S. L., Mitchell, J. T., \& Scott, M. S. (2000). Revealing the vulnerability of people and places: A case study of Georgetown County, South Carolina. Annals of the Association of American Geographers, 90(4), 713-737. doi: 10.1111/0004-5608.00219

Dannenberg, A. L., Jackson, R. J., Frumkin, H., Schieber, R. A., Pratt, M., Kochtitzky, C., \& Tilson, H. H. (2003). The impact of community design and land-use choices on public health: A scientific research agenda. American Journal of Public Health, 93(9), 15001508. doi: 10.2105/AJPH.93.9.1500

Davies, S. M., McDonald, K. M., Schmidt, E., Schultz, E., Geppert, J., \& Romano, P. S. (2009). Expanding use of the AHRQ prevention quality indicators. AHRQ.

Davis, K., Stremikis, K., Squires, D., \& Schoen, C. (2014). Mirror, mirror on the wall, 2014 update: How the U.S. health care system compares internationally (Vol. 16). New York: The Commonwealth Fund.

Deaton, A. (2007). Income, aging, health and wellbeing around the world: Evidence from the Gallup World Poll (NBER Working Paper No. 13317). National Bureau of Economic Research. 
Devaney, B., Haines, P., \& Moffitt, R. (1989). Assessing the dietary effects of the food stamp program. Volumes 1 and 2. Alexandria, VA: U.S. Department of Agriculture, Food and Nutrition Service.

DeVol, R., Bedroussian, A., Charuworn, A., Chatterjee, A., Kim, I. K., Kim, S., \& Klowden, K. (2007). An unhealthy America: The economic burden of chronic disease. Washington, DC: Milken Institute.

Diabetes Prevention Program Research Group. (2002). Reduction in the incidence of type 2 diabetes with lifestyle intervention or metformin. New England Journal of Medicine, 346(6), 393-403. doi: 10.1056/NEJMoa012512

Diabetes Prevention Program Research Group. (2009). 10-year follow-up of diabetes incidence and weight loss in the Diabetes Prevention Program Outcomes Study. The Lancet, 374(9702), 1677-1686. doi: 10.1016/S0140-6736(09)61457-4

Diener, E., \& Chan, M. Y. (2011). Happy people live longer: Subjective well-being contributes to health and longevity. Applied Psychology: Health and Well-Being, 3(1), 1-43. doi: 10.1111/j.1758-0854.2010.01045.x

Diener, E., Ng, W., Harter, J., \& Arora, R. (2010). Wealth and happiness across the world: Material prosperity predicts life evaluation, whereas psychosocial prosperity predicts positive feeling. Journal of Personality and Social Psychology, 99(1), 52-61. doi: $10.1037 / \mathrm{a} 0018066$

Diener, E., \& Seligman, M. E. (2004). Beyond money toward an economy of well-being. Psychological Science in the Public Interest, 5(1), 1-31. doi: 10.1111/j.09637214.2004.00501001.x

Diener, E., \& Suh, E. (1997). Measuring quality of life: Economic, social, and subjective indicators. Social Indicators Research, 40(1-2), 189-216. doi: 10.1023/A:1006859511756

Diez Roux, A. V., \& Mair, C. (2010). Neighborhoods and health. Annals of the New York Academy of Sciences, 1186(1), 125-145. doi: 10.1111/j.1749-6632.2009.05333.x

Ding, D., Sallis, J. F., Kerr, J., Lee, S., \& Rosenberg, D. E. (2011). Neighborhood environment and physical activity among youth: A review. American Journal of Preventive Medicine, 41(4), 442-455. doi: 10.1016/j.amepre.2011.06.036

diversitydatakids.org. (n.d.). Family and Medical Leave Act. As of February 24, 2016: http://www.diversitydatakids.org/data/policy/5/family-and-medical-leave-act

Docherty, I., Goodlad, R., \& Paddison, R. (2001). Civic culture, community and citizen participation in contrasting neighbourhoods. Urban Studies, 38(12), 2225-2250.

Dolan, P., Peasgood, T., \& White, M. (2008). Do we really know what makes us happy? A review of the economic literature on the factors associated with subjective well-being. Journal of Economic Psychology, 29, 94-122.

Dorfman, D. (1998). Mapping community assets workbook. Portland: Northwest Regional Educational Laboratory.

Dubowitz, T., Ghosh-Dastidar, M., Cohen, D. A., Beckman, R., Steiner, E. D., Hunter, G. P., . . Sloan, J. C. (2015). Diet and perceptions change with supermarket introduction in a food desert, but not because of supermarket use. Health Affairs, 34(11), 1858-1868. doi: 10.1377/hlthaff.2015.0667

Duncan, G. J., Dowsett, C. J., Claessens, A., Magnuson, K., Huston, A. C., Klebanov, P., . . . Japel, C. (2007). School readiness and later achievement. Developmental Psychology, 43(6), 1428-1446. doi: 10.1037/0012-1649.43.6.1428 
Duncan, M. J., Spence, J. C., \& Mummery, W. K. (2005). Perceived environment and physical activity: A meta-analysis of selected environmental characteristics. International Journal of Behavioral Nutrition and Physical Activity, 2(1), 11. doi: 10.1186/1479-5868-2-11

Dunn, J. R. (2000). Housing and health inequalities: Review and prospects for research. Housing Studies, 15(3), 341-366. doi: 10.1080/02673030050009221

Durand, C. P., Andalib, M., Dunton, G. F., Wolch, J., \& Pentz, M. A. (2011). A systematic review of built environment factors related to physical activity and obesity risk: Implications for smart growth urban planning. Obesity Reviews, 12(5), e173-e182. doi: 10.1111/j.1467-789X.2010.00826.x

Ebi, K. L., Sussman, F. G., \& Wilbanks, T. J. (2008). Analyses of the effects of global change on human health and welfare and human systems. Washington, DC: U.S. Environmental Protection Agency.

Econsult Corporation. (2013). The economic impacts of supermarkets on their surrounding communities (Vol. Issue 4). Philadelphia, PA: The Reinvestment Fund.

Edwards, J. B., Rauseo, M. S., \& Unger, K. R. (2013). Community centered: 23 reasons why your library is the most important place in town. Public Libraries.

Ehrlich, T. (2000). Civic responsibility and higher education. Westport, CT: Greenwood Publishing Group.

Eichstaedt, J. C., Schwartz, H. A., Kern, M. L., Park, G., Labarthe, D. R., Merchant, R. M., .. . Weeg, C. (2015). Psychological language on Twitter predicts county-level heart disease mortality. Psychological Science, 26(2), 159-169. doi: 10.1177/0956797614557867

Engeland, A., Tretli, S., \& Bjørge, T. (2004). Height and body mass index in relation to esophageal cancer; 23-year follow-up of two million Norwegian men and women. Cancer Causes \& Control, 15(8), 837-843.

Erickson, D., \& Andrews, N. (2011). Partnerships among community development, public health, and health care could improve the well-being of low-income people. Health Affairs (Millwood), 30(11), 2056-2063. doi: 10.1377/hlthaff.2011.0896

Feigin, V., Forouzanfar, M., Krishnamurthi, R., Mensah, G., Connor, M., Bennett, D., . . . Truelsen, T. (2014). Global Burden of Diseases, Injuries, and Risk Factors Study 2010 (GBD 2010) and the GBD Stroke Experts Group. Global and regional burden of stroke during 1990-2010: findings from the Global Burden of Disease Study 2010. The Lancet, 383(9913), 245-254.

Feinberg, L., Reinhard, S. C., Houser, A., \& Choula, R. (2011). Valuing the invaluable: 2011 update: The growing contributions and costs of family caregiving. AARP Public Policy Institute.

Felitti, V. J., Anda, R. F., Nordenberg, D., Williamson, D. F., Spitz, A. M., Edwards, V., . . . Marks, J. S. (1998). Relationship of childhood abuse and household dysfunction to many of the leading causes of death in adults: The Adverse Childhood Experiences (ACE) Study. American Journal of Preventive Medicine, 14(4), 245-258. doi: http://dx.doi.org/10.1016/S0749-3797(98)00017-8

Fisk, W. J. (2002). How IEQ affects health, productivity. ASHRAE Journal, 44(5), 56-56.

Flatau, P., Galea, J., \& Petridis, R. (2000). Mental health and wellbeing and unemployment. Australian Economic Review, 33(2), 161-181. doi: 10.1111/1467-8462.00145

Foege, W. H. (2010). Social determinants of health and health-care solutions. Public Health Reports, 125(Suppl 4), 8-10. 
Fox, S. E., Levitt, P., \& Nelson III, C. A. (2010). How the timing and quality of early experiences influence the development of brain architecture. Child Development, 81(1), 28-40.

Frank, L. D., Andresen, M. A., \& Schmid, T.L. (2004). Obesity relationships with community design, physical activity, and time spent in cars. American Journal of Preventive Medicine, 27(2):87-96.

Frank, L., \& Kavage, S. (2009). A national plan for physical activity: The enabling role of the built environment. Journal of Physical Activity \& Health, 6(2), S186-S195.

Frank, L. D., Sallis, J. F., Conway, T. L., Chapman, J. E., Saelens, B. E., \& Bachman, W. (2006). Many pathways from land use to health: Associations between neighborhood walkability and active transportation, body mass index, and air quality. Journal of the American Planning Association, 72(1), 75-87.

Friedberg, M. W., Lai, D. J., Hussey, P. S., \& Schneider, E. C. (2009). A guide to the medical home as a practice-level intervention. American Journal of Managed Care, 15(12), S291S299.

Friedland, D. S., \& Price, R. H. (2003). Underemployment: Consequences for the health and well-being of workers. American Journal of Community Psychology, 32(1-2), 33-45. doi: 10.1023/A:1025638705649

Friedman-Krauss, A., and Barnett, S. B. (2013, April). Early childhood education: Pathways to better health. NIEER Policy Brief, Vol. 25.

Furukawa, M. F., King, J., Patel, V., Hsiao, C. J., Adler-Milstein, J., \& Jha, A. K. (2014). Despite substantial progress in EHR adoption, health information exchange and patient engagement remain low in office settings. Health Affairs, 33(9), 1672-9679. doi: $10.1377 /$ hlthaff.2014.0445

Galea, S., Tracy, M., Hoggatt, K. J., DiMaggio, C., \& Karpati, A. (2011). Estimated deaths attributable to social factors in the United States. American Journal of Public Health, 101(8), 1456-1465. doi: 10.2105/AJPH.2010.300086

Galea, S., \& Vlahov, D. (2005). Urban health: Evidence, challenges, and directions. Annual Review of Public Health, 26, 341-365. doi: 10.1146/annurev.publhealth.26.021304.144708

Geertz, C. (1973). The interpretation of cultures: Selected essays (Vol. 5019). New York: Basic Books.

Gitlin, L. N., Belle, S. H., Burgio, L. D., Czaja, S. J., Mahoney, D., Gallagher-Thompson, D., . . . Schulz, R. (2003). Effect of multicomponent interventions on caregiver burden and depression: The REACH multisite initiative at 6-month follow-up. Psychology and Aging, 18(3), 361-374. doi: 10.1037/0882-7974.18.3.361

Graves, M. A., Harris, J. R., Hannon, P. A., Hammerback, K., Ahmed, F., \& Zhou, C. (2014). Workplace-based influenza vaccination promotion practices among large employers in the United States. Journal of Occupational and Environmental Medicine, 56(4), 397-402. doi: 10.1097/JOM.0000000000000115

Green, C. G., \& Klein, E. G. (2011). Promoting active transportation as a partnership between urban planning and public health: The Columbus Healthy Places program. Public Health Reports, 126(Suppl 1), 41-49.

Greenberg, M. R. (1999). Improving neighborhood quality: A hierarchy of needs. Housing Policy Debate, 10(3), 601-624. doi: 10.1080/10511482.1999.9521345 
Guo, S. S., \& Chumlea, W. C. (1999). Tracking of body mass index in children in relation to overweight in adulthood. American Journal of Clinical Nutrition, 70(1), 145s-148s.

Halfon, N., \& Conway, P. H. (2013). The opportunities and challenges of a lifelong health system. New England Journal of Medicine, 368(17), 1569-1571. doi: 10.1056/NEJMp1215897

Hamlin, C. (1998). Public health and social justice in the age of Chadwick: Britain, 1800-1854. Cambridge, UK: Cambridge University Press.

Handy, S. L., Boarnet, M. G., Ewing, R., \& Killingsworth, R. E. (2002). How the built environment affects physical activity: Views from urban planning. American Journal of Preventive Medicine, 23(2), 64-73. doi: 10.1016/S0749-3797(02)00475-0

Hannon, P. A., Harris, J. R., Sopher, C. J., Kuniyuki, A., Ghosh, D. L., Henderson, S., . . . Albano, D. L. (2012). Improving low-wage, midsized employers' health promotion practices: A randomized controlled trial. American Journal of Preventive Medicine, 43(2), 125-133. doi: 10.1016/j.amepre.2012.04.014

Harlow, R. E., \& Cantor, N. (1996). Still participating after all these years: A study of life task participation in later life. Journal of Personality and Social Psychology, 71(6), 1235.

Harris, J. R., Hannon, P. A., Beresford, S. A., Linnan, L. A., \& McLellan, D. L. (2014). Health promotion in smaller workplaces in the United States. Annual Review of Public Health, 35, 327-342. doi: 10.1146/annurev-publhealth-032013-182416

Health Research \& Educational Trust. (2014, October). Hospital-based strategies for creating a Culture of Health. Chicago. As of February 17, 2016: http://www.rwjf.org/en/library/research/2014/10/ hospital-based-strategies-for-creating-a-culture-of-health.html

Heath, B., Romero, P. W., \& Reynolds, K. (2013). A standard framework for levels of integrated healthcare. Washington, DC: SAMHSA-HRSA Center for Integrated Health Solutions.

Henrich, C. C., Schwab-Stone, M., Fanti, K., Jones, S. M., \& Ruchkin, V. (2004). The association of community violence exposure with middle-school achievement: A prospective study. Journal of Applied Developmental Psychology, 25(3), 327-348. doi: 10.1016/j.appdev.2004.04.004

Henry J. Kaiser Family Foundation. (2012). Emerging Medicaid accountable case organizations: The role of managed care. Menlo Park, CA.

HERO. (2015a). HERO employee health management best practicies scorecard in collaboration with Mercer: Annual report 2014. Edina, MN: HERO and Mercer.

HERO. (2015b, October 30). Culture of health measures: HERO Phase III preliminary report.

Hertz, E., Hebert, J. R., \& Landon, J. (1994, July). Social and environmental factors and life expectancy, infant mortality, and maternal mortality rates: Results of a cross-national comparison. Social Science \& Medicine, 39(1):105-114.

Herzog, A., Franks, M. M., Markus, H. R., \& Holmberg, D. (1998). Activities and well-being in older age: Effects of self-concept and educational attainment. Psychology and Aging, 13(2), 179.

Hillsdon, M., Panter, J., Foster, C., \& Jones, A. (2006). The relationship between access and quality of urban green space with population physical activity. Public Health, 120(12), 1127-1132. doi: 10.1016/j.puhe.2006.10.007

Hong, C., Siegel, A., \& Ferris, T. (2014). Caring for high-need, high-cost patients: What makes for a successful care management program? Commonwealth Fund Issue Brief. 
Howell, R. T., Kern, M. L., \& Lyubomirsky, S. (2007). Health benefits: Meta-analytically determining the impact of well-being on objective health outcomes. Health Psychology Review, 1(1). doi: 10.1080/17437190701492486

HRET - see Health Research \& Educational Trust.

Hume, C., Salmon, J., \& Ball, K. (2005). Children's perceptions of their home and neighborhood environments, and their association with objectively measured physical activity: A qualitative and quantitative study. Health Education Research, 20(1), 1-13. doi: 10.1093/her/cyg095

Huppert, F. A. (2009). Psychological well-being: Evidence regarding its causes and consequences. Applied Psychology: Health and Well-Being, 1(2), 137-164. doi: 10.1111/j.1758-0854.2009.01008.x

Huppert, F. A., \& So, T. T. C. (2013). Flourishing across Europe: Application of a new conceptual framework for defining well-being. Social Indicators Research, 110(3), 837861.

Iceland, J., \& Sharp, G. (2013). White residential segregation in U.S. metropolitan areas: Conceptual issues, patterns, and trends from the U.S. Census, 1980 to 2010. Population Research and Policy Review, 32(5), 10.1007/s11113-013-9277-6. http://doi.org/10.1007/s11113-013-9277-6

IHME — see Institute for Health Metrics and Evaluation.

Inglehart, R., \& Klingemann, H.-D. (2000). Genes, culture, democracy, and happiness. In E. Diener \& E. M. Suh (eds.), Culture and subjective well-being (pp. 165-183). Cambridge, MA: MIT Press.

Initiative for a Competitive Inner City. (2011, June). Creating shared value: Anchors and the inner city. As of January 19, 2016: http://www.icic.org/ee_uploads/images/Anchor_Paper_Web2.pdf

Inman, D. D., van Bakergem, K. M., Larosa, A. C., \& Garr, D. R. (2011). Evidence-based health promotion programs for schools and communities. American Journal of Preventive Medicine, 40(2), 207-219. doi: 10.1016/j.amepre.2010.10.031

Institute for Health Metrics and Evaluation. (2016). GBD publications. As of January 19, 2016: http://www.healthdata.org/node/961

Institute of Medicine. (1998). The future of public health. Washington, DC: National Academies Press.

Institute of Medicine. (2001). Crossing the quality chasm: A new health system for the 21st century. Washington, DC: National Academies Press.

Institute of Medicine. (2002). Care without coverage: Too little, too late. Washington, DC: National Academies Press.

Institute of Medicine. (2010). Future of nursing. Washington, DC: National Academies Press. Institute of Medicine. (2012). Primary care and public health: Exploring integration to improve population health. Washington, DC: National Academies Press.

Institute of Medicine. (2013). New directions: What we've learned about strategies and interventions for child abuse and neglect. Washington, DC: National Academies Press.

Institute of Medicine. (2014a). Capturing social and behavioral domains in electronic health records: Summary of selected domains. Washington, DC: National Academies Press.

Institute of Medicine. (2014b). Financing population health improvement: Workshop summary. Washington, DC: National Academies Press. 
Institute of Medicine Committee on the Future Health Care Workforce for Older Americans. (2008). Retooling for an aging America: Building the health care workforce. Washington, DC: National Academies Press.

Isaacs, J. B. (2012). Starting school at a disadvantage: The school readiness of poor children. The Social Genome Project. Washington, DC: Brookings Institution.

Jackson, S. A., Anderson, R. T., Johnson, N. J., \& Sorlie, P. D. (2000). The relation of residential segregation to all-cause mortality: A study in black and white. American Journal of Public Health, 90(4), 615-617.

Jamison, E. A., Jamison, D. T., \& Hanushek, E. A. (2007). The effects of education quality on income growth and mortality decline. Economics of Education Review, Elsevier, 26(6), 771-788.

Joshi, P., Geronimo, K., Romano, B., Earle, A., \& Acevedo-Garcia, D. (2015). Family and Medical Leave Act policy equity assessment. Waltham, MA: Institute for Child, Youth and Family Policy, Heller School for Social Policy \& Management, Brandeis University.

Juszczak, L., Schlitt, J., and Moore, A. (2007, May). 2004-2005 National School-Based Health Care Census. Washington, DC: National Assembly on School-Based Health Care. As of February 17, 2016:

http://www.sbh4all.org/wp-content/uploads/2015/06/ 2004-NASBHC-Census-Brochure.pdf

Juszczak, L., Schlitt, J., Odlum, M., Barangan, C., \& Washington, D. (2003, May). 2001-2002 National School-Based Health Care Census. Washington, DC: National Assembly on School-Based Health Care. As of February 17, 2016: http://www.sbh4all.org/wp-content/uploads/2015/06/Census-data-report.pdf

Kabir, Z., Arora, M., \& Alpert, H. R. (2011). Have public smoking bans resulted in an improvement in quality of life? Expert Review of Pharmacoeconomics and Outcomes Research, 11(4), 375-377.

Kahneman, D., Krueger, A. B., Schkade, D. A., Schwarz, N., \& Stone, A. A. (2004). A survey method for characterizing daily life experience: The day reconstruction method. Science, 306(5702), 1776-1780. doi: 10.1126/science.1103572

Kapucu, N., Arslan, T., \& Demiroz, F. (2010). Collaborative emergency management and national emergency management network. Disaster Prevention and Management, 19(4), 452-468.

Kawachi, I., Kennedy, B. P., Lochner, K., \& Prothrow-Stith, D. (1997). Social capital, income inequality, and mortality. American Journal of Public Health, 87(9), 1491-1498. doi: 10.2105/AJPH.87.9.1491

Kawachi, I., Subramanian, S. V., \& Kim, D. (2008). Social capital and health. New York: Springer.

Keeler, E. B., Brook, R. H., Goldberg, G. A., Kamberg, C. J., \& Newhouse, J. P. (1985). How free care reduced hypertension in the health insurance experiment. JAMA, 254(14), 19261931.

Keeton, V., Soleimanpour, S., \& Brindis, C. D. (2012). School-based health centers in an era of health care reform: Building on history. Current Problems in Pediatric and Adolescent Health Care, 42(6), 132-156. doi: 10.1016/j.cppeds.2012.03.002

Kelly, C. M., Schootman, M., Baker, E. A., Barnidge, E. K., \& Lemes, A. (2007). The association of sidewalk walkability and physical disorder with area-level race and 
poverty. Journal of Epidemiology and Community Health, 61(11), 978-983. doi: 10.1136/jech.2006.054775

Khanani, I., Elam, J., Hearn, R., Jones, C., \& Maseru, N. (2010). The impact of prenatal WIC participation on infant mortality and racial disparities. American Journal of Public Health, 100(S1), S204-S209. doi: 10.2105/AJPH.2009.168922

Kickbusch, I., \& Gleicher, D. (2012). Governance for health in the 21st century. Copenhagen: World Health Organization Regional Office for Europe.

Kim, I. H., Muntaner, C., Khang, Y. H., Paek, D., \& Cho, S. I. (2006). The relationship between nonstandard working and mental health in a representative sample of the South Korean population. Social Science \& Medicine, 63(3), 566-574.

Kimmelman, M. (2013, October 2). Next time, libraries could be our shelters from the storm. New York Times. As of February 18, 2016: http:/www.nytimes.com/2013/10/03/arts/design/ next-time-libraries-could-be-our-shelters-from-the-storm.html?_r=0

Kindig, D., \& Stoddart, G. (2003). What is population health? American Journal of Public Health, 93(3), 380-383. doi: 10.2105/AJPH.93.3.380

Kivimäki, M., Vahtera, J., Virtanen, M., Elovainio, M., Pentti, J., \& Ferrie, J. E. (2003). Temporary employment and risk of overall and cause-specific mortality. American Journal of Epidemiology, 158(7), 663-668.

Kleinman, J. C., Feldman, J. J., \& Mugge, R. H. (1976). Geographic variations in infant mortality. Public Health Reports, 91(5), 423-432.

Komro, K. A., Tobler, A. L., Delisle, A. L., O’Mara, R. J., \& Wagenaar, A. C. (2013). Beyond the clinic: Improving child health through evidence-based community development. $B M C$ Pediatrics, 13, 172. doi: 10.1186/1471-2431-13-172

Kott, P., Hedden, S., Aldworth, J., Bose, J., Chromy, J., Gfroerer, J., . . . Abuse, S. (2013). 2012 National Survey on Drug Use and Health: A revised strategy for estimating the prevalence of mental illness. SAMHSA.

Kovalchik, S. A., Elliott, M. N., Quigley, D. D., \& Martin, L. (under review). Measuring geographic variation in patient experience of care: A multi-setting regional index of health care experience. Health Affairs.

Kramer, M. R., \& Hogue, C. R. (2009). Is segregation bad for your health? Epidemiologic Review, 31(1), 178-194. doi: 10.1093/epirev/mxp001

Kreider, B., Pepper, J. V., Gundersen, C., \& Jolliffe, D. (2012). Identifying the effects of SNAP (Food Stamps) on child health outcomes when participation is endogenous and misreported. Journal of the American Statistical Association, 107(499), 958-975. doi: $10.1080 / 01621459.2012 .682828$

Krieger, J., \& Higgins, D. L. (2002). Housing and health: Time again for public health action. American Journal of Public Health, 92(5), 758-768. doi: 10.2105/AJPH.92.5.758

Kroeber, A. L., \& Kluckhohn, C. (1952). Culture: A critical review of concepts and definitions. Cambridge, MA: Peabody Museum of Archaeology \& Ethnology.

Kruk, J. (2007). Physical activity in the prevention of the most frequent chronic diseases: An analysis of the recent evidence. Asian Pacific Journal of Cancer Prevention, 8(3), 325327.

Kuo, F. E. (2001). Coping with poverty impacts of environment and attention in the inner city. Environment and Behavior, 33(1), 5-34. 
Kuo, F. E., \& Sullivan, W. C. (2001). Aggression and violence in the inner city effects of environment via mental fatigue. Environment and Behavior, 33(4), 543-571.

Lasker, R. D., \& Weiss, E. S. (2003a). Broadening participation in community problem solving: A multidisciplinary model to support collaborative practice and research. Journal of Urban Health, 80(1), 14-47. doi: 10.1093/jurban/jtg014

Lasker, R. D., \& Weiss, E. S. (2003b). Creating partnership synergy: The critical role of community stakeholders. Journal of Health and Human Services Administration, 26(1), 119-139.

Lasker, R. D., Weiss, E. S., \& Miller, R. (2001). Partnership synergy: A practical framework for studying and strengthening the collaborative advantage. Milbank Quarterly, 79(2), 179205. doi: 10.1111/1468-0009.00203

Laverack, G. (2006). Improving health outcomes through community empowerment: A review of the literature. Journal of Health, Population and Nutrition, 24(1), 113-120.

Lavizzo-Mourey, R. (2014, February 10). Building a Culture of Health; President's message. Robert Wood Johnson Foundation. As of February 9, 2016: http://www.rwjf.org/en/about-rwjf/annual-reports/presidents-message-2014.html

Lawrence, R. G. (2004). Framing obesity: The evolution of news discourse on a public health issue. Harvard International Journal of Press-Politics, 9(3), 56-75. doi: 10.1177/1081180X04266581

Lear, J. G., Eichner, N., \& Koppelman, J. (1999). The growth of school-based health centers and the role of state policies: Results of a national survey. Archives of Pediatrics and Adolescent Medicine, 153(11), 1177-1180. doi: 10.1001/archpedi.153.11.1177

LeClere, F. B., Rogers, R. G., \& Peters, K. (1997). Ethnicity and mortality in the United States: Individual and community correlates. Social Forces, 76:169-198.

Lee, H. (2012). The role of local food availability in explaining obesity risk among young school-aged children. Social Science \& Medicine, 74(8), 1193-1203. doi: 10.1016/j.socscimed.2011.12.036

Leppo, K., Ollila, E., Pena, S., Wismar, M., \& Cook, S. (2013). Health in all policies: Seizing opportunities, implementing policies. Helsinki, Finland: Ministry of Social Affairs and Health. As of February 18, 2016:

http://www.euro.who.int/_data/assets/pdf_file/0007/188809/ Health-in-All-Policies-final.pdf

Link, B. G., \& Phelan, J. (1995). Social conditions as fundamental causes of disease. Journal of Health and Social Behavior, 35, 80-94.

Littman, T. (2010). Evaluating public transportation health benefits. Victoria, British Columbia: Transport Policy Institute.

Lofink, H., Kuebler, J., Juszczak, L., Schlitt, J., Even, M., Rosenberg, J., \& White, I. (2013). 2010-2011 school-based health alliance census report. Washington, DC: School-Based Health Alliance. As of February 9, 2016: http://www.sbh4all.org/wp-content/uploads/2015/02/ CensusReport_2010-11CensusReport_7.13.pdf

Long, S., Karpman, M., Shartzer, A., Wissokerer, D., Kenney, G. M., Zuckerman, S., . . . Hempstead, K. (2014). Taking stock: Health insurance coverage under the ACA as of September 2014. Washington, DC: Urban Institute.

Long, S. K., Kenney, G. M., Zuckerman, S., Wissoker, D., Shartzer, A., Karpman, M., \& Anderson, N. (2014). Quicktake: Number of uninsured adults continues to fall under the 
ACA: Down by 8.0 million in June 2014. Washington, DC: Urban Institute, Health Policy Center.

Lu, M. C., \& Halfon, N. (2003). Racial and ethnic disparities in birth outcomes: A life-course perspective. Maternal and Child Health Journal, 7(1), 13-30. doi: 10.1023/A:1022537516969

Ludwig, J., Duncan, G. J., Gennetian, L. A., Katz, L. F., Kessler, R. C., Kling, J. R., \& Sanbonmatsu, L. (2012). Neighborhood effects on the long-term well-being of lowincome adults. Science, 337(6101), 1505-1510. doi: 10.1126/science.1224648

Lund, H. (2002). Pedestrian environments and sense of community. Journal of Planning Education and Research, 21(3), 301-312.

Lund, H. (2003). Testing the claims of new urbanism: Local access, pedestrian travel, and neighboring behaviors. Journal of the American Planning Association, 69(4), 414-429.

Lupton, D. (1992). Discourse analysis: A new methodology for understanding the ideologies of health and illness. Australian Journal of Public Health, 16(2), 145-150. doi: 10.1111/j.1753-6405.1992.tb00043.x

Lyubomirsky, S., Sheldon, K. M., \& Schkade, D. (2005). Pursuing happiness: The architecture of sustainable change. Review of General Psychology, 9(2), 111-131. doi: 10.1037/10892680.9.2.111

Maas, J., Verheij, R. A., Groenewegen, P. P., De Vries, S., \& Spreeuwenberg, P. (2006). Green space, urbanity, and health: How strong is the relation? Journal of Epidemiology and Community Health, 60(7), 587-592. doi: 10.1136/jech.2005.043125

Maas, J., Verheij, R. A., Spreeuwenberg, P., \& Groenewegen, P. P. (2008). Physical activity as a possible mechanism behind the relationship between green space and health: A multilevel analysis. BMC Public Health, 8(1), 206. doi: 10.1186/1471-2458-8-206

Maben, J., Cornwell, J., \& Sweeney, K. (2010). In praise of compassion. Journal of Research in Nursing, 15(1), 9-13. doi: 10.1177/1744987109353689

Martin, L., \& Luoto, J. E. (2015). From coverage to care: Strengthening and facilitating consumer connections to the health system (PE-158-CMS). Santa Monica, CA: RAND Corporation. As of February 29, 2016: http://www.rand.org/pubs/perspectives/PE158.html

Mathews, T., \& MacDorman, M. F. (2010). Infant mortality statistics from the 2006 period linked birth/infant death data set. National Vital Statistics Reports, 58(17), 1-31.

Mays, G. P., \& Hogg, R. A. (2015). Economic shocks and public health protections in US metropolitan areas. American Journal of Public Health, 105 Suppl 2, S280-287. doi: 10.2105/ajph.2014.302456

Mays, G. P., Scutchfield, F. D., Bhandari, M. W., \& Smith, S. A. (2010). Understanding the organization of public health delivery systems: An empirical typology. Milbank Quarterly, 88(1), 81-111. doi: 10.1111/j.1468-0009.2010.00590.x

McCarthy, D., Klein, S., Cohen, A., \& Fund, C. (2014). The road to accountable care: Building systems for population health management. Washington, DC: Commonwealth Fund.

McMillan, D. W., \& Chavis, D. M. (1986). Sense of community: A definition and theory. Journal of Community Psychology, 14(1), 6-23.

McQueen, D. V., Wismar, M., Lin, V., Jones, C. M., \& Davies, M. (2012). Intersectoral governance for health in all policies: Structures, actions and experiences. Brussels: European Observatory on Health Systems and Policies. 
McWilliams, M. J. (2009). Health consequences of uninsurance among adults in the United States: Recent evidence and implications. Milbank Quarterly, 87(2), 443-494.

Mechanic, D., \& Tanner, J. (2007). Vulnerable people, groups, and populations: Societal view. Health Affairs, 26(5), 1220-1230. doi: 10.1377/hlthaff.26.5.1220

Mehta, N. B., \& Partin, M. H. (2007). Electronic health records: A primer for practicing physicians. Cleveland Clinic Journal of Medicine, 74(11), 826-830.

MetLife. (2011). The MetLife study of cargiving costs to working caregivers. New York.

Mitchell, L., Frank, M. R., Harris, K. D., Dodds, P. S., \& Danforth, C. M. (2013). The geography of happiness: Connecting Twitter sentiment and expression, demographics, and objective characteristics of place. PLOS One, 8(5), e64417. doi: 10.1371/journal.pone.0064417

Mitchell, R., \& Popham, F. (2008). Effect of exposure to natural environment on health inequalities: An observational population study. The Lancet, 372(9650), 1655-1660. doi: 10.1016/S0140-6736(08)61689-X

Mokdad, A. H., Marks, J. S., Stroup, D. F., \& Gerberding, J. L. (2004). Actual causes of death in the United States, 2000. JAMA, 291(10), 1238-1245. doi: 10.1001/jama.291.10.1238

Molassiotis, A., Wilson, B., Blair, S., Howe, T., \& Cavet, J. (2011). Unmet supportive care needs, psychological well-being and quality of life in patients living with multiple myeloma and their partners. Psycho-Oncology, 20(1), 88-97. doi: 10.1002/pon.1710

Moreland-Russell, S., Eyler, A., Barbero, C., Hipp, J. A., \& Walsh, H. (2013). Diffusion of Complete Streets policies across US communities. Journal of Public Health Management and Practice, 19, S89-S96. doi: 10.1097/PHH.0b013e3182849ec2

Morrow, V. (2001). Using qualitative methods to elicit young people's perspectives on their environments: Some ideas for community health initiatives. Health Education Research, 16(3), 255-268. doi: 10.1093/her/16.3.255

Muhlestein, D. (2013, October 29). What, really, is an ACO? Leavitt Partners. As of February 17, 2016: http://leavittpartners.com/2013/10/really-aco/

Muhlestein, D. (2014, January 29). Accountable care growth in 2014: A look ahead. Health Affairs Blog. As of January 8, 2016: http://healthaffairs.org/blog/2014/01/29/accountable-care-growth-in-2014-a-look-ahead/

Murray, C. J., Vos, T., Lozano, R., Naghavi, M., Flaxman, A. D., Michaud, C., . . Memish, Z. A. (2012). Disability-adjusted life years (DALYs) for 291 diseases and injuries in 21 regions, 1990-2010: A systematic analysis for the Global Burden of Disease Study 2010. The Lancet, 380(9859), 2197-2223. doi: 10.1016/s0140-6736(12)61689-4

NACCHO_see National Association of County and City Health Officials.

National Alliance for Caregiving \& AARP Public Policy Institute. (2015). Executive summary: Caregiving in the U.S. Bethesda, MD: National Alliance for Caregiving.

National Association of County and City Health Officials. (2009). 2008 national profile of local health departments. Washington, DC.

National Association of County and City Health Officials. (2014, January). 2013 national profile of local health departments. Washington, DC. As of February 17, 2016: http://nacchoprofilestudy.org/

National Center for Education Statistics. (2006). The health literacy of America's adults: Results from the 2003 National Assessment of Adult Literacy. Washington, DC: U.S. Department of Education.

National Collaborative on Childhood Obesity Research. (2014). Annual Report 2014. 
National Complete Streets Coalition. (2015). The many benefits of complete streets. As of January 8, 2016:

http://www.smartgrowthamerica.org/complete-streets/complete-streets-fundamentals/ benefits-of-complete-streets/

National Education Association. (2004a). The effects of state public K-12 education expenditures on income distribution. NEA Working Research Paper.

National Education Association. (2004b). K-12 education in the U.S. economy: Its impact on economic development, earnings and housing values. NEA Working Research Paper.

National Education Association. (2004c). School funding, taxes and economic growth: An analysis of the 50 states. NEA Working Research Paper.

National Institute for Early Education Research. (2004). The state of preschool. As of February 18, 2016: http://nieer.org/sites/nieer/files/2004yearbook.pdf

National Research Council. (2011). Explaining divergent levels of longevity in high-income countries. Crimmins, E.M., Preston, S.H., \& Cohen, B. (eds.), Panel on Understanding Divergent Trends in Longevity in High-Income Countries. Committee on Population, Division of Behavioral and Social Sciences and Education. Washington, DC: National Academies Press.

National Research Council and Institute of Medicine. (2013). U.S. health in international perspective: Shorter lives, poorer health. Washington, DC: National Academies Press. doi:10.17226/13497 As of February 18, 2016:

http://www.nap.edu/catalog/13497/

us-health-in-international-perspective-shorter-lives-poorer-health

National Scientific Council on the Developing Child. (2010). Persistent fear and anxiety can affect young children's learning and development. Working Paper No. 9. Cambridge, MA: Harvard University. As of February 17, 2016:

http://developingchild.harvard.edu/wp-content/uploads/2010/05/

Persistent-Fear-and-Anxiety-Can-Affect-Young-Childrens-Learning-and-

Development.pdf

National Scientific Council on the Developing Child. (2012). The science of neglect: The persistent absence of responsive care disrupts the developing brain. Working Paper 12. Cambridge, MA: Harvard University. As of February 17, 2016 :

http://developingchild.harvard.edu/wp-content/uploads/2012/05/

The-Science-of-Neglect-The-Persistent-Absence-of-Responsive-Care-Disrupts-theDeveloping-Brain.pdf

National Survey of Child and Adolescent Well-Being. (2013). National survey of child and adolescent well-being: Child well-being spotlights. As of January 21, 2016: http://www.acf.hhs.gov/programs/opre/resource/ national-survey-of-child-and-adolescent-well-being-ii-nscaw-ii-child-well

NEA—see National Education Association.

Neckerman, K. M., Lovasi, G. S., Davies, S., Purciel, M., Quinn, J., Feder, E., . . Rundle, A. (2009). Disparities in urban neighborhood conditions: Evidence from GIS measures and field observation in New York City. Journal of Public Health Policy, 30, S264-S285. doi: 10.1057/jphp.2008.47

NIEER — see National Institute for Early Education Research. 
Norris, F. H., Stevens, S. P., Pfefferbaum, B., Wyche, K. F., \& Pfefferbaum, R. L. (2008). Community resilience as a metaphor, theory, set of capacities, and strategy for disaster readiness. American Journal of Community Psychology, 41(1-2), 127-150. doi: 10.1007/s10464-007-9156-6

O’Campo, P., Xue, X., Wang, M. C., \& Caughy, M. (1997, July). Neighborhood risk factors for low birthweight in Baltimore: A multilevel analysis. American Journal of Public Health, 87(7):1113-1118.

O’Mara-Eves, A., Brunton, G., McDaid, G., Oliver, S., Kavanagh, J., Jamal, F., . . Thomas, J. (2013). Community engagement to reduce inequalities in health: A systematic review, meta-analysis and economic analysis. Public Health Research, 1(4).

O’Malley, A. S., Grossman, J. M., Cohen, G. R., Kemper, N. M., \& Pham, H. H. (2010). Are electronic medical records helpful for care coordination? Experiences of physician practices. Journal of General Internal Medicine, 25(3), 177-185. doi: 10.1007/s11606009-1195-2

Oakley, P. (1999). Community involvement in health development: An examination of the critical issues. Geneva: World Health Organization.

OECD_see Organisation for Economic Co-operation and Development.

Organisation for Economic Co-operation and Development. (2013). Health at a glance 2013: OECD indicators. Paris.

Pant, A. T., Kirsch, T. D., Subbarao, I. R., Hsieh, Y.-H., \& Vu, A. (2008). Faith-based organizations and sustainable sheltering operations in Mississippi after Hurricane Katrina: Implications for informal network utilization. Prehospital and Disaster Medicine, 23(1), 48-54. doi: 10.1017/S1049023X00005550

Panter, J. R., \& Jones, A. P. (2008). Associations between physical activity, perceptions of the neighbourhood environment and access to facilities in an English city. Social Science \& Medicine, 67(11), 1917-1923. doi: 10.1016/j.socscimed.2008.09.001

Patterson, C., \& Chambers, L. (1995). Preventive health care. The Lancet, 345(8965), 16111615. doi: 10.1016/S0140-6736(95)90119-1

Peluso, M. A. M., \& Andrade, L. H. S. G. d. (2005). Physical activity and mental health: The association between exercise and mood. Clinics, 60(1), 61-70. doi: 10.1590/S180759322005000100012

Petersen, P. E., Bourgeois, D., Ogawa, H., Estupinan-Day, S., \& Ndiaye, C. (2005). The global burden of oral diseases and risks to oral health. Bulletin of the World Health Organization, 83(9), 661-669. doi: 10.1590/S0042-96862005000900011

Pfefferbaum, B. J., Reissman, D. B., Pfefferbaum, R. L., Klomp, R. W., \& Gurwitch, R. H. (2005). Building resilience to mass trauma events. In Doll, L. S., Bonzo, S. E., Mercy, J. A., \& Sleet, D. A. (eds.), Handbook on injury and violence prevention interventions. New York: Kluwer Academic Publishers.

Pickett, K. E., \& Pearl, M. (2001). Multilevel analyses of neighbourhood socioeconomic context and health outcomes: A critical review. Journal of Epidemiology \& Community Health, 55(2), 111-122.

Pinquart, M., \& Sörensen, S. (2003). Associations of stressors and uplifts of caregiving with caregiver burden and depressive mood: A meta-analysis. Psychological Sciences and Social Sciences, 58(2), P112-P128. doi: 10.1093/geronb/58.2.P112

Plough, A., Chandra, A., Leviton, L., Miller, C., Orleans, C. T., Trujillo, M., ... Yeung, D. (2015). From vision to action: A framework and measures to mobilize a Culture of 
Health. Robert Wood Johnson Foundation. As of February 29, 2015:

http://www.rwjf.org/content/dam/files/rwjf-web-files/Research/2015/

RWJF_From_Vision_to_Action_2015-FullReport.pdf

Podobnik, B. (2002). New urbanism and the generation of social capital: Evidence from Orenco Station. National Civic Review, 91(3), 245-255.

Pol, L. G., \& Thomas, R. K. (2000). The demography of health and health care. New York: Plenum Press.

Polednak, A. P. (1996, May). Trends in U.S. urban black infant mortality, by degree of residential segregation. American Journal of Public Health. 86(5):723-726.

Pollack, C. E., Griffin, B. A., \& Lynch, J. (2010). Housing affordability and health among homeowners and renters. American Journal of Preventive Medicine, 39(6), 515-521. doi: 10.1016/j.amepre.2010.08.002

Portes, A. (1998). Social capital: Its origins and applications in modern sociology. Annual Review in Sociology, 24, 1-24.

Pressman, S. D., \& Cohen, S. (2005). Does positive affect influence health? Psychological Bulletin, 131(6), 925-971. doi: 10.1037/0033-2909.131.6.925

Price, R. A., \& Elliott, M. (2014, December 10). The great patient experience survey myth. The Health Care Blog. As of February 18, 2016:

http://thehealthcareblog.com/blog/2014/12/10/

debunking-the-patient-experience-survey-myth/

Pronk, N. P., Baase, C., Noyce, J., \& Stevens, D. E. (2015). Corporate America and community health: Exploring the business case for investment. Journal of Occupational and Environmental Medicine, 57(5), 493-500. doi: 10.1097/JOM.0000000000000431

Putnam, R. D. (2000). Bowling Alone: The Collapse and Revival of American Community. New York: Simon \& Schuster.

Raina, P., O’Donnell, M., Rosenbaum, P., Brehaut, J., Walter, S. D., Russell, D., .. . Wood, E. (2005). The health and well-being of caregivers of children with cerebral palsy. Pediatrics, 115(6), e626-e636.

Ranji, S. R., Rennke, S., \& Wachter, R. M. (2013, March). Computerized provider order entry with clinical decision support systems: Brief update review. In Making health care safer II: An updated critical analysis of the evidence for patient safety practices. Rockville, MD: Agency for Healthcare Research and Quality (Evidence Reports/Technology Assessments, No. 211, Chapter 41). As of February 18, 2016: http:/www.ncbi.nlm.nih.gov/books/NBK133383/

The Reinvestment Fund. (2013). Access to supermarkets in inner-city communities. Reinvestment Brief (Issue 5). Oakland, CA.

Ribisl, K. M., Lee, R. E., Henriksen, L., \& Haladjian, H. H. (2003). A content analysis of web sites promoting smoking culture and lifestyle. Health Education \& Behavior, 30(1), 6478. doi: $10.1177 / 1090198102239259$

Riley, G. F., \& Lubitz, J. D. (2010). Long-term trends in Medicare payments in the last year of life. Health Services Research, 45(2), 565-576.

Rittenhouse, D. R., Thom, D. H., \& Schmittdiel, J. A. (2010). Developing a policy-relevant research agenda for the patient-centered medical home: A focus on outcomes. Journal of General Internal Medicine, 25(6), 593-600. doi: 10.1007/s11606-010-1289-x

Robert Wood Johnson Foundation. (2011). Does food and beverage marketing influence children's food choices? Princeton, NJ. 
Robert Wood Johnson Foundation. (2014). The relative contribution of multiple determinants to health outcomes. Health Policy Brief.

Rongen, A., Robroek, S. J., van Lenthe, F. J., \& Burdorf, A. (2013). Workplace health promotion: A meta-analysis of effectiveness. American Journal of Preventive Medicine, 44(4), 406-415. doi: 10.1016/j.amepre.2012.12.007

Rose, G. (1992). The strategy of preventive medicine. New York: Oxford University Press.

Rosen, G. (1993). A history of public health, expanded edition. Baltimore, MD: Johns Hopkins University Press.

Roussos, S. T., \& Fawcett, S. B. (2000). A review of collaborative partnerships as a strategy for improving community health. Annual Review of Public Health, 21(1), 369-402. doi: 10.1146/annurev.publhealth.21.1.369

Roy, M., \& Payette, H. (2006). Meals-on-wheels improves energy and nutrient intake in a frail free-living elderly population. The Journal of Nutrition, Health \& Aging, 10(6), 554-560.

RTI International. (2011). Accountable care organization 2012 program analysis quality performance standards narrative measure specifications. Waltham, MA.

Rudin, R. S. (2010). The litmus test for health information exchange success: Will small practices participate? Comment on "health information exchange." Archives of Internal Medicine, 170(7), 629-630. doi: 10.1001/archinternmed.2010.59

Rudin, R. S., Salzberg, C. A., Szolovits, P., Volk, L. A., Simon, S. R., \& Bates, D. W. (2011). Care transitions as opportunities for clinicians to use data exchange services: How often do they occur? Journal of the American Medical Informatics Association, 18(6), 853-858. doi: 10.1136/amiajnl-2010-000072

Saarni, S. I., Härkänen, T., Sintonen, H., Suvisaari, J., Koskinen, S., Aromaa, A., \& Lönnqvist, J. (2006). The impact of 29 chronic conditions on health-related quality of life: A general population survey in Finland using 15D and EQ-5D. Quality of Life Research, 15(8), 1403-1414. doi: 10.1007/s11136-006-0020-1

Saleem, J. J., Flanagan, M., Militello, L. G., Arbuckle, N., Russ, A. L., Burgo-Black, A. L., \& Doebbeling, B. N. (2011). Paper persistence and computer-based workarounds with the electronic health record in primary care. Paper presented at the Proceedings of the Human Factors and Ergonomics Society Annual Meeting.

Sallis, J. F., Frank, L. D., Saelens, B. E., \& Kraft, M. K. (2004). Active transportation and physical activity: Opportunities for collaboration on transportation and public health research. Transportation Research Part A: Policy and Practice, 38(4), 249-268. doi: 10.1016/j.tra.2003.11.003

Sallis, J. F., Slymen, D. J., Conway, T. L., Frank, L. D., Saelens, B. E., Cain, K., \& Chapman, J. E. (2011). Income disparities in perceived neighborhood built and social environment attributes. Health \& Place, 17(6), 1274-1283. doi: 10.1016/j.healthplace.2011.02.006

SAMHSA - see Substance Abuse and Mental Health Services Administration.

Sampson, R. J. (2003). The neighborhood context of well-being. Perspectives in Biology and Medicine, 46(3), S53-S64. doi: 10.1353/pbm.2003.0073

Schlitt, J., Santelli, J., Juszczak, L., Brindis, C., Nystrom, R., Klein, J., Kaplan, D., and Seibou, M. D. (2000, June). Creating access to care: School-Based Health Center Census 199899. Washington, DC: National Assembly on School-Based Health Care. As of February 18, 2016: http://www.sbh4all.org/wp-content/uploads/2015/08/1998-Census-Report.pdf 
Schneider, K. M., O’Donnell, B. E., \& Dean, D. (2009). Prevalence of multiple chronic conditions in the United States' Medicare population. Health Quality Life Outcomes, 7, 82. doi: 10.1186/1477-7525-7-82

Schwartz, H. A., Eichstaedt, J., Kern, M. L., Park, G., Sap, M., Stillwell, D., . . Ungar, L. (2014). Towards assessing changes in degree of depression through Facebook. Paper presented at the Proceedings of the Workshop on Computational Linguistics and Clinical Psychology: From Linguistic Signal to Clinical Reality, Baltimore, MD.

Seligman, M. E. (2002). Positive psychology, positive prevention, and positive therapy. New York: Oxford University Press.

Seville, W. (2009). Libraries bringing values to our communities. Planning Commissioners Journal, 75.

Seymour, R. A., Preshaw, P. M., \& Steele, J. G. (2002). Oral health and heart disease. Primary Dental Care, 9(4), 125-130. doi: 10.1308/135576102322481947

Sheiham, A., \& Watt, R. G. (2000). The common risk factor approach: A rational basis for promoting oral health. Community Dentistry and Oral Epidemiology, 28(6), 399-406. doi: 10.1034/j.1600-0528.2000.028006399.x

Shouls, S., Congdon, P., \& Curtis, S. (1996, June). Modelling inequality in reported long term illness in the UK: combining individual and area characteristics. Journal of Epidemiology and Community Health, 50(3):366-376.

Shumacher Center for a New Economics. (2011). Measuring for the future: An overview of measurements of progress and sustainability on the state-level. As of January 8, 2016: http://www.centerforneweconomics.org/publications/ measuring-future-overview-measurements-progress-and-sustainability-state-level

Sleddens, E. F., Kroeze, W., Kohl, L. F., Bolten, L. M., Velema, E., Kaspers, P., . . Brug, J. (2015). Correlates of dietary behavior in adults: An umbrella review. Nutrition Reviews, 73(8), 477-499. doi: 10.1093/nutrit/nuv007

Smart Growth America. (2016). Complete streets fundamentals. As of February 15, 2016: http://www.smartgrowthamerica.org/complete-streets/ complete-streets-fundamentals\#presentation

Smith, J. C. (2004). Alterations in brain and immune function produced by mindfulness meditation: Three caveats. Psychosomatic Medicine, 66(1), 148-149.

Solís-Vivanco, R., Mondragón-Maya, A., León-Ortiz, P., Rodríguez-Agudelo, Y., Cadenhead, K. S., \& de la Fuente-Sandoval, C. (2014). Mismatch negativity reduction in the left cortical regions in first-episode psychosis and in individuals at ultra high-risk for psychosis. Schizophrenia Research, 158(1), 58-63.

Sommers, B. D., Musco, T., Finegold, K., Gunja, M. Z., Burke, A., \& McDowell, A. M. (2014). Health reform and changes in health insurance coverage in 2014. New England Journal of Medicine, 371(9), 867-874. doi: 10.1056/NEJMsr1406753

Sprangers, M. A., de Regt, E. B., Andries, F., van Agt, H. M., Bijl, R. V., de Boer, J. B., . . . Kempen, G. I. (2000). Which chronic conditions are associated with better or poorer quality of life? Journal of Clinical Epidemiology, 53(9), 895-907. doi: 10.1016/S08954356(00)00204-3

Stange, K. C., Nutting, P. A., Miller, W. L., Jaén, C. R., Crabtree, B. F., Flocke, S. A., \& Gill, J. M. (2010). Defining and measuring the patient-centered medical home. Journal of General Internal Medicine, 25(6), 601-612. doi: 10.1007/s11606-010-1291-3 
Stewart, G. T., Kolluru, R., \& Smith, M. (2009). Leveraging public-private partnerships to improve community resilience in times of disaster. International Journal of Physical Distribution and Logistics Management, 39(5), 343-364.

Stoto, M. A. (2013a). Community health needs assessments: An opportunity to bring public health and the healthcare delivery system together to improve population health. Improving Population Health. As of January 14, 2016: http://www.improvingpopulationhealth.org/blog/2013/04/ community-health-needs-assessments-an-opportunity-to-bring-public-health-and-thehealthcare-delivery.html

Stoto, M. A. (2013b, February 21). Population health in the Affordable Care Act era. AcademyHealth.

Stoto, M. A., Abel, C., \& Dievler, A. (1996). Healthy communities: New partnerships for the future of public health. Washington, DC: National Academies Press.

Strozer, J., Juszczak, L., \& Ammerman, A. (2010). 2007-2008 National School-Based Health Care Census. Washington, DC: National Assembly on School-Based Health Care. As of February 17, 2016:

http://www.sbh4all.org/wp-content/uploads/2015/06/ NASBHC-2007-08-Census-Report-Final.pdf

Sturm, R., \& Cohen, D. (2014). Proximity to urban parks and mental health. Journal of Mental Health Policy and Economics, 17(1), 19-24.

Subramanian, S., Kim, D., \& Kawachi, I. (2005). Covariation in the socioeconomic determinants of self rated health and happiness: A multivariate multilevel analysis of individuals and communities in the USA. Journal of Epidemiology and Community Health, 59(8), 664669. doi: 10.1136/jech.2004.025742

Substance Abuse and Mental Health Services Administration, Center for Behavioral Health Statistics and Quality. (2013, January). The NSDUH report: Trends in insurance coverage and treatment utilization by young adults. Rockville, MD. As of February 17, 2016: http://www.samhsa.gov/data/sites/default/files/SR-1887/SR-1887.pdf

Swidler, A. (1986). Culture in action: Symbols and strategies. American Sociological Review, 51(2), 273-286.

Talen, E. (1999). Sense of community and neighbourhood form: An assessment of the social doctrine of new urbanism. Urban Studies, 36(8), 1361-1379.

Tan, C. E., \& Glantz, S. A. (2012). Association between smoke-free legislation and hospitalizations for cardiac, cerebrovascular, and respiratory diseases: A meta-analysis. Circulation, 126(18), 2177-2183. doi: 10.1161/CIRCULATIONAHA.112.121301

Taylor, M. (2000). Communities in the lead: Power, organisational capacity and social capital. Urban Studies, 37(5/6), 1019.

Taylor, Z. E., Doane, L. D., \& Eisenberg, N. (2013). Transitioning from high school to college: Relations of social support, ego-resiliency, and maladjustment during emerging adulthood. Emerging Adulthood, 2(2), 105-115. doi: 10.1177/2167696813506885

Teicher, M. H., Anderson, C. M., \& Polcari, A. (2012). Childhood maltreatment is associated with reduced volume in the hippocampal subfields CA3, dentate gyrus, and subiculum. Proceedings of the National Academy of Sciences, 109(9), E563-572.

Teno, J. M., Gozalo, P. L., Bynum, J. P., Leland, N. E., Miller, S. C., Morden, N. E., . . Mor, V. (2013). Change in end-of-life care for Medicare beneficiaries: Site of death, place of care, 
and health care transitions in 2000, 2005, and 2009. JAMA, 309(5), 470-477. doi: 10.1001/jama.2012.207624

Thomson, H., Petticrew, M., \& Douglas, M. (2003). Health impact assessment of housing improvements: Incorporating research evidence. Journal of Epidemiology and Community Health, 57(1), 11-16. doi: 10.1136/jech.57.1.11

Tinetti, M. E., Fried, T. R., \& Boyd, C. M. (2012). Designing health care for the most common chronic condition - multimorbidity. JAMA, 307(23), 2493-2494. doi: 10.1001/jama.2012.5265

Tomlinson, M., Rotheram-Borus, M. J., Swartz, L., \& Tsai, A. C. (2013). Scaling up mHealth: Where is the evidence? PLOS One, 10(2), e1001382. doi: 10.1371/journal.pmed.1001382

Trochim, W. M., \& Linton, R. (1986). Conceptualization for planning and evaluation. Evaluation and Program Planning, 9(4), 289-308. doi: 10.1016/0149-7189(86)90044-3

Trochim, W. M., Milstein, B., Wood, B. J., Jackson, S., \& Pressler, V. (2004). Setting objectives for community and systems change: An application of concept mapping for planning a statewide health improvement initiative. Health Promotion Practice, 5(1), 8-19. doi: $10.1177 / 1524839903258020$

Turner, S., Merchant, K., Kania, J., \& Martin, E. (2012). Understanding the value of backbone organizations in collective impact: Part 1. Standford Social Innovation Review.

U.S. Census Bureau. (2010). Homepage. As of January 21, 2016: http://www.census.gov/

U.S. Department of Agriculture, Economic Research Service. (2013, March 1). Food Access Research Atlas: About the Atlas. As of February 15, 2016: http://www.ers.usda.gov/data-products/food-access-research-atlas/about-the-atlas.aspx

U.S. Department of Health and Human Services. (2006). The health consequences of involuntary exposure to tobacco smoke: A report of the Surgeon General. Secondhand Smoke-What It Means to You. U.S. Department of Health and Human Services, Centers for Disease Control and Prevention, Coordinating Center for Health Promotion, National Center for Chronic Disease Prevention and Health Promotion, Office on Smoking and Health.

U.S. Department of Housing and Urban Development. (n.d.[a]). Affordable housing. As of February 15, 2016: http://portal.hud.gov/hudportal/HUD?src=/program_offices/comm_planning/ affordablehousing/

U.S. Department of Housing and Urban Development. (n.d.[b]). Moving to Opportunity for fair housing. As of February 15, 2016: http://portal.hud.gov/hudportal/HUD?src=/programdescription/mto

U.S. Department of Transportation. (2009, February). Assessing the full costs of congestion on surface transportation systems and reducing them through pricing. As of February 18, 2016: https://www.transportation.gov/sites/dot.dev/files/docs/ Costs\%20of\%20Surface\%20Transportation\%20Congestion.pdf

U.S. Department of Transportation. (2015). Transportation and health tool. As of January 19, 2016: https://www.transportation.gov/transportation-health-tool

Umberson, D., \& Montez, J. K. (2010). Social relationships and health: A flashpoint for health policy. Journal of Health and Social Behavior 51(1), S54-S66. doi: $10.1177 / 0022146510383501$ 
UnitedHealth Group. (2013). Doing good is good for you: 2013 health and volunteering study. Minnetonka, MN. As of February 17, 2016:

http://www.unitedhealthgroup.com/ /media/uhg/pdf/2013/

unh-health-volunteering-study.ashx

United Nations Development Programme (UNDP). (2014). Homepage. As of January 21, 2016: http://www.undp.org/

United States Global Change Research Program. (2009). Global climate change impacts in the United States. New York.

University of Wisconsin Population Health Institute. (2014). County health rankings key findings.

Uscher-Pines, L., Chandra, A., \& Acosta, J. (2013). The promise and pitfalls of community resilience. Disaster Medicine and Public Health Preparedness, 7(6), 603-606. doi: $10.1017 / \mathrm{dmp} .2013 .100$

USDA Food and Nutrition Service. (2013a). National School Lunch Program.

USDA Food and Nutrition Service. (2013b, November 18, 2013). Women, Infants and Children (WIC): About WIC-How WIC helps. As of November 9, 2015: http://www.fns.usda.gov/wic/about-wic-how-wic-helps

Van Dijk, T. A. (1993). Principles of critical discourse analysis. Discourse \& Society, 4(2), 249283.

Varda, D. M., Forgette, R., Banks, D., \& Contractor, N. (2009). Social network methodology in the study of disasters: Issues and insights prompted by post-Katrina research. Population Research and Policy Review, 28, 11-29.

Ver Ploeg, M. (2012). Access to affordable and nutritious food: Updated estimates of distance to supermarkets using 2010 data. United States Department of Agriculture, Economic Research Service.

Verba, S., Nie, N. H., \& Kim, J. (1978). Political participation and political equality: A sevennation comparison. New York and London: Cambridge University Press.

W.K. Kellogg Foundation. (2007). The collective leadership framework: A workbook for cultivating and sustaining community change. Battle Creek, MI.

Wagner, E. H., Austin, B. T., \& Von Korff, M. (1996). Organizing care for patients with chronic illness. Milbank Quarterly, 74(4), 511-544. doi: 10.2307/3350391

Ward, B. W., Schiller, J. S., \& Goodman, R. A. (2014). Multiple chronic conditions cmong US adults: A 2012 update. Preventing Chronic Disease, 11(E62). doi: 10.5888/pcd11.130389

Warr, P. (1988). Work, jobs and unemployment: Item 8. SET: Research Information for Teachers.

Weisburd, D., \& Eck, J. E. (2004). What can police do to reduce crime, disorder, and fear? Annals of the American Academy of Political and Social Science, 593(1), 42-65.

Weisburd, D., Bennett, T., Gill, C., \& Telep, C. (2012). Community-oriented policing to reduce crime, disorder and fear and increase legitimacy and citizen satisfaction in neighborhoods. Oslo, Norway: Campbell Collaboration.

Whetten, K., Ostermann, J., Whetten, R. A., Pence, B. W., O’Donnell, K., Messer, L. C., \& Thielman, N. M. (2009). A comparison of the wellbeing of orphans and abandoned children ages 6-12 in institutional and community-based care settings in 5 less wealthy nations. PLoS One, 4(12), e8169. doi: 10.1371/journal.pone.0008169 
Whitaker, R. C., Wright, J. A., Pepe, M. S., Seidel, K. D., \& Dietz, W. H. (1997). Predicting obesity in young adulthood from childhood and parental obesity. New England Journal of Medicine, 337(13), 869-873. doi: 10.1056/NEJM199709253371301

White, L. A. (1949). The science of culture, a study of man and civilization. New York: Farrar, Straus.

Whittemore, R. (2000). Strategies to facilitate lifestyle change associated with diabetes mellitus. Journal of Nursing Scholarship, 32(3), 225-232.

WHO_ see World Health Organization.

Wholey, D. R., Gregg, W., \& Moscovice, I. (2009). Public health systems: A social networks perspective. Health Services Research, 44(5p2), 1842-1862. doi: 10.1111/j.14756773.2009.01011.x

Willard, R., Shah, G. H., Leep, C., \& Ku, L. (2012). Impact of the 2008-2010 economic recession on local health departments. Journal of Public Health Management and Practice, 18(2), 106-114. doi: 10.1097/PHH.0b013e3182461cf2

Williams, D. R., \& Collins, C. (1995). US socioeconomic and racial differences in health: Patterns and explanations. Annual Review of Sociology, 349-386.

Wiseman, J., \& Brasher, K. (2008). Community wellbeing in an unwell world: Trends, challenges, and possibilities. Journal of Public Health Policy, 29(3), 353-366. doi: 10.1057/jphp.2008.16

Witt, W. P., Park, H., Wisk, L. E., Cheng, E. R., Mandell, K., Chatterjee, D., \& Zarak, D. (2015). Neighborhood disadvantage, preconception stressful life events, and infant birth weight. American Journal of Public Health, 105(5), 1044-1052. doi: 10.2105/AJPH.2015.302566

Wolf, M. S., Gazmararian, J. A., \& Baker, D. W. (2007). Health literacy and health risk behaviors among older adults. American Journal of Preventive Medicine, 32(1), 19-24. doi: 10.1016/j.amepre.2006.08.024

World Health Organization. (n.d.). The Ottawa Charter for health promotion. As of February 17, 2016: http://www.who.int/healthpromotion/conferences/previous/ottawa/en/

World Health Organization. (1948). Constitution of the World Health Organization. Paper presented at the International Health Conference, New York.

World Health Organization. (2005). Ecosystems and human well-being: Health synthesis. Geneva, Switzerland.

World Health Organization. (2006). Health in all policies: Prospects and potentials. Geneva, Switzerland.

Yach, D., Hawkes, C., Gould, C. L., \& Hofman, K. J. (2004). The global burden of chronic diseases: Overcoming impediments to prevention and control. JAMA, 291(21), 26162622. doi: 10.1001/jama.291.21.2616

Yong-Chan, K., \& Jinae, K. (2010, April). Communication, neighbourhood belonging and household hurricane preparedness. Disasters, 34(2):470-488.

Young, P. L., Saunders, R., \& Olsen, L. (2010). The healthcare imperative: Lowering costs and improving outcomes: Workshop series summary. Washington, DC: National Academies Press.

Zahner, S. J., \& Corrado, S. M. (2004). Local health department partnerships with faith-based organizations. Journal of Public Health Management and Practice, 10(3), 258-265.

Zatzick, D. F., Marmar, C. R., Weiss, D. S., Browner, W. S., Metzler, T. J., Golding, J. M., . . . Wells, K. B. (2014). Posttraumatic stress disorder and functioning and quality of life 
outcomes in a nationally representative sample of male Vietnam veterans. American Journal of Psychiatry, 154(12), 1690-1695. doi: 10.1176/ajp.154.12.1690

Zickuhr, K., Rainie, H., Purcell, K., \& Duggan, M. (2013). How Americans value public libraries in their communities. Washington, DC: Pew Research Center. 


\section{Appendix A. Frequently Asked Questions About the Culture of Health}

This appendix summarizes key questions often posted about the Culture of Health, the action framework, and associated measures.

\section{Overview and Purpose of Action Framework}

\section{What is the origin of the action framework?}

In 2013, RWJF proposed a vision of America in which we all strive together to build a national Culture of Health that enables all in our diverse society to lead healthier lives, now and for generations to come. Ten underlying principles provided the foundation for this vision and the inspiration for the action framework, developed to mobilize a national Culture of Health and achieve an outcome of improved population health, well-being, and equity.

\section{How was the action framework developed?}

The action framework and its components - the action areas, drivers, and measures - are drawn from rigorous research and analysis of the systemic problems holding the United States back from a higher level of health. RWJF developed the action framework in collaboration with the RAND Corporation and with valuable input from more than 1,000 experts, partners, colleagues, community members, focus group participants, and global leaders during a two-year period.

There were three analytic steps used to move from the ten Culture of Health principles to the action framework. First, we examined how principles may cluster together. Team members independently organized the principles into clusters, then reviewed and named these clusters or action areas. After the principles were grouped, the team created logic models to determine how action areas would link together to drive or influence the Culture of Health. Ultimately, we collapsed those models to create the single action framework. The exercise ensured that our choice of groupings aligned with available evidence and conceptual clarity. Finally, as part of the extensive engagement efforts with diverse stakeholders, we checked our assumptions about the action areas, specifically to determine whether the conceptual groupings we had recommended were useful and understood by a diverse set of stakeholders across the country.

\section{Why is the action framework needed?}

Despite ongoing efforts to improve the health of our nation, positive change is not occurring at a promising pace. The Culture of Health action framework is intended to be broad and strategic, setting a national agenda and mobilizing a movement to improve health, well-being, and equity. The framework includes an integrated, evidence-based, and comprehensive approach to addressing the societal influences of health and well-being. In doing so, it calls for unprecedented collaboration between individuals; organizations in the private and public sectors; and communities representing a range of social, demographic, and geographic characteristics. 


\section{What is the purpose of the From Vision to Action report?}

The report (Plough et al., 2015) is meant to describe the urgent need to catalyze change in health, well-being, and equity. The Culture of Health action framework is intended to spur dialogue and collaborative action between individuals, communities, and organizations across the public and private sectors. Additional research and other supporting materials will be developed to further outline RWJF's strategy and specific activities, and also to show how communities are embracing the framework. The Culture of Health website will provide an additional platform for sharing new information and promising practices.

\section{Details of Action Framework}

\section{Why use the term action framework?}

Unlike a model — which implies something formulaic, fixed, and final - $\mathrm{a}$ framework speaks to a built-in fluidity. The action framework invites individuals, communities, and organizations to utilize the structure and find entry points relevant to their unique needs and goals. The framework is not a blueprint but, rather, suggests multiple pathways for action.

\section{Why is equity an overarching theme?}

In order to truly build a Culture of Health, we cannot leave anyone behind. Everyone should have the opportunity to pursue the healthiest life possible, regardless of where they live or work, the color of their skin, or the amount of money they have. This is why achieving health equity is a goal that permeates the entire action framework.

\section{The Culture of Health term seems somewhat abstract. Why use the term culture?}

Culture allows us to reflect all of the deep components that drive social change. We define culture as the sharing and alignment of beliefs, attitudes, values, and actions across a set of individuals, organizations, and decision environments (e.g., where policies or laws are made). It follows, then, that a Culture of Health is a set of social ideas and practices that promote healthy individuals, households, neighborhoods, communities, states, and nations. These shared ideas and practices are key drivers for whether and how sectors work together to improve health environments. We should note that there is not necessarily a single, common culture. Rather, cultures can develop in communities, as defined by geographic boundaries and demographic characteristics. In communities with a strong Culture of Health, we expect to observe individuals valuing good health and making healthy choices about their physical, mental, spiritual, and social well-being, and we expect organizations and the environment to support those healthy choices. A brief review of successful, large-scale social change (e.g., drastic reductions in highway deaths and smoking and increases in recycling) suggests that these changes were not the result of any single intervention or even of a small set of interventions. Instead, these shifts arose from many different kinds of actors at all levels of society pulling the levers that were available to them because each actor had a vested interest in contributing to the desired outcomes. 


\section{What is the key difference between the action areas, drivers, and measures?}

The action areas and drivers constitute the essential and enduring structure of the action framework. They establish the priorities we should focus on as nation, and they will remain constant over time. The measures are adaptable and will evolve to keep pace with changing conditions.

\section{Details of Measures}

\section{How were the Culture of Health measures selected?}

The measures are intended to be illustrative indicators of where our nation needs to improve in order to advance a Culture of Health. The measures have been rigorously identified as having the evidence base necessary to lead to improved health and well-being. Through an intensive process of screening and winnowing, an initial list of hundreds of potential measures was honed down to the set included in the report. Individual measures were reviewed using criteria such as relevance, validity, and potential for collaborative action. Then the full set was reviewed for its cohesiveness in broadly increasing health equity, while also considering whether major national health challenges, such as obesity, were addressed. The measures include protective factors that, if increased, would improve health; they also include risk factors that, if reduced, would improve health and well-being. They are intended to be clear and understandable-empowering various audiences to make positive changes in health behaviors and policies.

Additionally, the measures are intended to

- inspire dialogue and action

- highlight priority areas that have eluded our focus as a nation but which are crucial to building a Culture of Health

- encourage a broader view of health, incorporating all aspects of well-being

- welcome sectors not traditionally associated with health or health care services, encouraging cross-sector collaboration

- provide entry points for individuals, families, and communities across diverse populations

- focus on both drivers and outcomes of health and well-being

- include data that are accessible and represent powerful signals of change.

\section{Why were these 41 measures selected? What was the process? What were the criteria?}

The team conducted an extensive process to review and select potential measures. This included a literature review to inform development of the Culture of Health action framework, including selection of action areas, drivers, and measure areas. In addition, the team used stakeholder analysis, including concept mapping and other qualitative techniques, to further refine the model and, ultimately, the measures. The team also engaged in several iterative, decisionmaking processes to review potential measures and winnow the list. Criteria for measures selection included the following:

- Relevance. The measure can apply to one or more Culture of Health action areas.

- Validity. The measure is tested or at least has good face and content validity.

- Signal value, catalytic value, and feed-forward value. The measure would provide good information on national and/or local Culture of Health progress to inform intervention/improvement processes.

- Potential for collaboration. The measure would support or galvanize partnership between two or more sectors (with RWJF where relevant), moving activity in a sector on behalf of a Culture of 
Health.

- Accessible/understandable by range of audiences. Measures, individually and as a group, can be explained to and readily understood by users. The measures will resonate with audiences.

- Balance across outcomes, drivers, and important contextual factors. Measures represent balance across the influencers and the results of action.

- Mixture of risk and protective factors. Measures reflect Culture of Health vulnerabilities and assets.

\section{How did you/will you validate these measures?}

We will be working nationally and with a sample of communities (called sentinel communities) to review the validity of measures. For example, we may conduct small studies to determine how well our hospital partnership measure signals collaboration and collective action for community health. RWJF will also devote some research funding to furthering the science surrounding these measures. However, RWJF also invites the broader research community to offer its methods for measures validation through existing or future studies.

\section{Over what period of time will you track these measures? Five years? Ten years? Longer?}

We will track progress in the action framework over at least a generation, approximately 20 years. This means using the measures to assess how well the nation is moving toward a Culture of Health over that time period. However, we expect that the exact measures will evolve over time as the nation adopts the Culture of Health action framework. The action framework will not change, but measures may change as new data are available or significant progress is made in a particular area of the action framework.

\section{How are measures likely to evolve over time?}

Currently, we are including measures based on the criteria noted earlier (signal value, validity, etc.), but we are also using measures for which data are currently available. We hope that as the Culture of Health action framework permeates national dialogue, we can start to work on what available data we have in the action areas, or drivers and measure areas. This will ensure that we have measures that further capture the signal or catalytic value to chart progress in a Culture of Health. For example, we may include stable health insurance as a measure now, given current political and social context, but as insurance coverage become less of a national concern, this may evolve into another measure of access. The action framework and the first set of measures may highlight the need for new and different types of data and measures to track progress. Additionally, RWJF will be supporting ongoing and new areas of research to further build the evidence base to support the Culture of Health model, and that research will likely identify new measures.

\section{How do these measures address issues of disparities and inequities in the drivers of health and well- being?}

Issues of equity apply to many of the Culture of Health measures, and addressing societal disparities and inequities is a pivotal goal in building a Culture of Health. Every measure speaks to being able to better address health and health care disparities, and equity is in the title of action area 3 (creating healthier, more equitable community environments). The Culture of Health framework and measures are put forward, in part, with the intention of eliminating health disparities based on the social, 
environmental, and economic determinants of health and well-being. We intend to disaggregate and present data on age, race/ethnicity, socioeconomic status, gender, and geography whenever possible.

How is this set of measures different from existing national metrics, such as Healthy People 2020, America's Health Rankings, and the Institute of Medicine's Vital Signs?

The Culture of Health measures have been purposely selected to build upon such efforts by highlighting underlying factors that influence health not represented in other national reports. The measures are uniquely focused on tracking upstream social, economic, and policy indicators that, if improved, should significantly enhance population health and well-being on a national level. The Institute of Medicine and federal health officials recognize how these measures align with their work to improve national health metrics and how these measures advance the way our nation gauges actions aimed at addressing the social determinants of health. The measures use a variety of data sources and methodologies by design - not just to engage different sectors, but also to reflect the complexity of decisionmaking and the multipronged ways communities get things done. For example, this is the only set of measures that uniquely combines broad influences, from business investment to early childhood development to urban design. While some measures directly address health care services and the patient experience, many reflect underlying societal issues that influence health and well-being. The measures speak to the importance of social support and community, public discussions around health promotion, and policies that facilitate collaboration across sectors.

\section{How do the Culture of Health measures complement the County Health Rankings \& Roadmaps?}

The development of the national measures was closely aligned with the framework used for RWJF's "sister set" of data, the County Health Rankings \& Roadmaps. Like the county health data, the measures presented in this report highlight many factors that influence health. However, we did not duplicate any of the County Health Rankings measures, which serve the specific purpose of ranking counties along standard health outcomes to facilitate local dialogue and action.

The Culture of Health measures address specific, systemic factors that drive health at a national level, broaden the dialogue about what cross-sector actions improve health, and inform the evolution of local and national improvement efforts.

\section{How do you actually measure "culture" using this set of measures?}

The complexity of culture as a concept requires us to review the full set of measures together to determine the extent to which all sectors and actors are working in concert with each other. But that only tells a part of the national story. We will also employ our analyses in 30 sentinel communities to examine the ways in which a Culture of Health evolves at a local level, using such methodologies as key informant interviews, ethnography, and policy review. These analyses will ensure that we can assess what factors are influencing cultural change and the extent to which a community is realizing all action areas of the Culture of Health action framework. 


\section{End Users for Measures}

\section{Who do you see as principal audiences for these measures?}

We anticipate that these measures will be used by a wide variety of stakeholders and sectors, since every sector has a role in building a Culture of Health; this is a shared American responsibility. As such, we hope that leaders in all communities and sectors will review the measures and the action framework and find their work represented. RWJF will help to lead a part of the dialogue on how different sectors can become involved, but it cannot be the sole voice.

The audiences include (1) thought leaders (to include researchers, policymakers, and philanthropic leaders), (2) program architects (to include city and regional planners and community development organizations), and (3) advocacy groups (to include grant makers) - in sum, many of those with whom we already work.

\section{How can individuals and families use the action framework?}

In a Culture of Health, individuals and families play an important part in spearheading sustainable local change. The action framework is intended to inspire, strengthen, and support individuals, families, caregivers, and communities in making healthier choices and engaging in healthier behavior. Individuals and families should review the action framework and consider the ways in which they are promoting better health and well-being. Individuals and families can engage with their communities and promote the kind of collective action that is needed.

\section{How can public- and private-sector organizations use the action framework?}

Because every sector has a role in building a Culture of Health, we anticipate that a wide variety of stakeholders and sectors will use the action framework. As such, it is our hope that leaders across communities and sectors will review the action framework, see their work reflected in it, and contribute to it through their own efforts and ingenuity. The framework builds upon the energy and legacy of those who have worked to improve health, well-being, and equity for years. It also opens the door to new allies and provides entry points showing the transformative roles they can play — or may already be playing — in this national effort.

\section{RWJF Uses of the Action Framework and Measures}

\section{How will RWJF use the action framework and these measures?}

The Culture of Health action framework describes the broad aims and actionable elements of a longterm effort of RWJF, both in catalyzing national action and guiding our own investments. We intend that this framework will develop and guide our work over the next 20 years, with annual updates.

Additionally, RWJF developed a user-friendly, interactive website (www.cultureofhealth.org). This will be a go-to source for deeper analysis of the measures, including detailed breakdowns by race/ethnicity, income levels, and geography, where possible. The website will connect individuals and communities from across the nation, providing information and tools that they can use in their own settings. It will also offer opportunities to join in conversation about the action framework, the corresponding measures, and the overall vision of building a Culture of Health with researchers and policymakers. 


\section{What do you intend to do with the measures? Will there be a national report each year?}

We will update the information behind these measures as quickly as data are refreshed (generally every year or two). As new measures are included to replace those in the first set of measures, we will provide updates on our national progress for a Culture of Health. We will also provide annual updates and spotlights on how different sectors are engaging with the Culture of Health action framework and what strategies they are employing. The Culture of Health website will also be updated regularly.

\section{What does this mean for funding by RWJ in the future? Will you fund studies on the measures or using the measures, even ones on housing or transportation?}

While RWJF does not see itself as the exclusive driver of change for any of the specific measures, many RWJF research and action programs will incorporate these and related measures in their efforts, as goals or focus areas for programming or measurement. Several new research programs and solicitations make use of these measures to assess and help catalyze progress toward a Culture of Health and toward greater equity in opportunities for health and well-being. New surveys will help to clarify how U.S. residents in varied communities think about, prioritize, and value health and how they view community cohesion and its impact on health and well-being.

RWJF has been supporting action programs, demonstrations, and research in most of the areas and subareas of the Culture of Health action framework. For instance, the foundation has supported analysis through its past and present work to inform and support actions to improve individual and community health, health care, and public health services (e.g., childhood obesity prevention; public health systems and services research; health care quality; cross-cutting efforts to reduce disparities in health and wellbeing; and access to the social, environmental and economic drivers of health and well-being in multiple sectors).

\section{What are next steps for the measures?}

The specific action areas and critical subareas are intended to provide a robust focus for action by many persons and organizations. The specific measures we have selected for this 1.0 version have an evidence base linking improvement in these indicators to improved health or well-being. However, these are starting points for broader consideration of other related measures that might be more significant and more actionable for specific community or sector. We will substitute some different measures in our reports going forward, as we learn more about what most powerfully improves the broader action framework.

\section{Involvement with the Culture of Health}

\section{Is the foundation partnering with other groups or organizations to apply, evolve, and learn from these} measures?

Yes, RWJF is working with many national and local organizations to review and test measures. The Culture of Health work relies on these networks to inform how the measures will evolve and for providing key insights for the action framework. 


\section{I want to learn more—how do I get more information? How can I (or my organization) get involved?}

The launch of the metrics was accompanied by the report From Vision to Action (Plough et al., 2015), which provides a description of all of the measures and the ways in which they link to improved health and well-being. In the fall of 2015, we launched the Culture of Health website, which provides more data for each measure, data visualizations, and stories and narratives about how a Culture of Health is emerging across the country in sentinel communities. The website also includes links to county health rankings (county-level data and roadmaps to action) and RWJF's Datahub (state-level data), as well as other data and toolkit linkages. If you would like to get involved, there are several ways to share stories and other information via the Culture of Health website.

I want to contribute to this work using measures my team or organization has been developing. Are there ways to collaborate?

We are seeking input from you and your organization. There will be several ways to contribute insight. First, if you are a member of the RWJF leadership network, we will maintain a forum for ideas and recommendations. Second, the Culture of Health website will include forms for offering insights into measures and sharing stories and lessons learned from applying the action framework. We also are open to your ideas regarding how we may collaborate to advance a Culture of Health in your organization and your sector.

\section{Will the actual dataset be available for public use and analysis?}

The data will be made available; however, because the data for these measures come from a wide variety of sources (mostly publicly available), were collected using different data collection methods, are in different formats, and cover different time periods, creating one dataset is not possible. The Culture of Health website will provide data or links to the data. Data from the Culture of Health survey will be made available on the website. We also will make the survey instrument and data set available on RWJF's Health and Medical Care Archive at the Interuniversity Consortium of Political and Social Research (ICPSR). 


\section{Appendix B. Sentinel Community Overview and Frequently Asked Questions}

This appendix includes a brief overview of the sentinel community purpose and design via a frequently asked questions document describing the sentinel community process.

\section{What is the Culture of Health Initiative?}

The Robert Wood Johnson Foundation (RWJF) has embarked on a bold new strategy to achieve a Culture of Health in America, a culture that enables all in our diverse society to lead healthier lives now and for generations to come. Building a comprehensive Culture of Health has become the central aim of our research and our investments. (See RWJF's 2015 Annual Report for more information and detail on our new vision.) In the 2014 annual message, Building a Culture of Health, RWJF presents ten underlying principles that provide both the foundation and the goal for the Culture of Health.

1. Good health flourishes across geographic, demographic, and social sectors.

2. Attaining the best health possible is valued by our entire society.

3. Individuals and families have the means and the opportunity to make choices that lead to the healthiest lives possible.

4. Business, government, individuals, and organizations work together to build healthy communities and lifestyles.

5. Everyone has access to affordable, quality health care because it is essential to maintain, or reclaim, health.

6. No one is excluded.

7. Health care is efficient and equitable.

8. The economy is less burdened by excessive and unwarranted health care spending.

9. Keeping everyone as healthy as possible guides public and private decisionmaking.

10. Americans understand that we are all in this together.

Our nation is splendidly diverse, and a national movement toward better health must reflect our individual beliefs, our family customs, and our community values. When the United States ultimately achieves a Culture of Health, it will be as multifaceted as the population it serves.

\section{What is the Culture of Health action framework?}

In collaboration with the RAND Corporation - and with valuable input from partners and colleagues across the country - RWJF has developed a framework to mobilize our nation's progress toward building a Culture of Health. By clustering the ten principles into four action areas, we established an action framework to chart and catalyze progress toward the outcome of improved population health and wellbeing for all.

The Culture of Health action framework is a long-term initiative that will guide RWJF's work and investments over the next 20 years. The entire action framework is designed to give special attention to equity and not to merely describe the health disparities that exist, but to eliminate them. 
Each action area is accompanied by a set of national measures, intended to inspire a national movement and also provide a menu of possible actions at the local level. Rather than being definitive, the measures are intended as starting points for action and conversation about the many factors that influence and improve health. They will illustrate progress and evolve to keep pace with changing needs.

The measures will provide an overarching view of the nation's progress toward the goal of building a Culture of Health for all. However, we understand that transformative activities are currently being developed and implemented in communities around the country and that fundamental change toward a Culture of Health needs to occur at a local level. This is why we are embarking on a complementary effort in the Culture of Health sentinel communities to understand how individual communities are developing a Culture of Health and how local activities align with our action framework.

\section{What is a sentinel community, and why observe a community?}

A sentinel community is a geographically defined community (city, county, metropolitan statistical area, or state) that is selected for the purpose of observing how a Culture of Health takes hold and evolves at the local level. Sentinel communities will provide a window into the various ways communities are bringing the Culture of Health action framework to life and will allow us to amplify successes and refine the measures over time. These communities will serve as a national sample group, enabling RWJF to track changes being made in real time and identify signals of progress in building a Culture of Health.

\section{Why have you invited my community? Why this set of communities?}

The 30 sentinel communities will reflect diversity by geography, demographic features, approaches to improving the health of their community, and collective action to improve well-being across sectors. There is no single way to build a Culture of Health, and many approaches may provide valuable insight on how to make progress toward achieving an outcome of improved population health and well-being.

\section{What kinds of activities will this Culture of Health sentinel community effort involve?}

Sentinel community participation will be limited to data collection only. Selection as a sentinel community is independent from and not linked to any other grant funding decision by RWJF (past, current, or future). Sentinel communities may or may not have current grant funding from RWJF, and future RWJF funding is not constrained or enhanced by being selected a sentinel community.

Data collection will be coordinated with a community point of contact (likely the lead public health entity for the jurisdiction). Some data collection will be from existing data and will not require any new data collection activity or assistance from your community. Data will include some administrative information on health, social, and economic indicators; interviews with community leaders regarding their activities to support or advance a Culture of Health; and policy review regarding plans and programs that may support aspects of a Culture of Health. In some communities, additional activities may include analysis of local surveys, media, or other data.

\section{What is the benefit of being a sentinel community?}

As a sentinel community, you will be at the forefront of informing how a Culture of Health develops and evolves. Lessons and insights from your community may be applicable to many other settings. Your community's experiences, challenges, and ideas will be shared broadly. For your community's efforts to 
collect or share data or information, we will provide sufficient monetary support to cover all data collection costs. We will share all collected data and analysis with the lead health department point of contact in the community, but there will be no ranking or rating of sentinel communities in any way. Aggregate information from all sentinel communities will be linked to an interactive Culture of Health website, designed to connect individuals and communities from across the nation by providing tools they can use in their own settings.

\section{How long will being a sentinel community last?}

We will work with the local health department or lead public health entity (and other organizations

locally, as needed) to collect data on an annual basis for at least five years. This effort is not intended to require significant involvement of community stakeholders each year.

\section{How will data collection be coordinated?}

RWJF will work with a national coordinating organization, which will oversee data collection responsibilities. Data collection will occur via staff of the national coordinator. Teams deployed by the national coordinator will work with the sentinel communities, principally through the local health department or lead public health entity, to gather administrative data or organize primary data collection (e.g., key informant interviews). 
Because health is a function of more than medical care, solutions to U.S. health problems must encompass more than reforms to health care systems. But those working to improve health, well-being, and equity still too often find themselves traveling on parallel paths that rarely intersect. In 2013, the Robert Wood Johnson Foundation (RWJF) embarked on a pioneering effort to advance a Culture of Health. A Culture of Health places well-being at the center of every aspect of life, with the goal of enabling everyone in our diverse society to lead healthier lives, now and for generations to come. To put this vision into action, RWJF worked with RAND to develop an action framework that identifies how the nation will work toward achieving these outcomes. This report provides background on the development of this action framework. The Culture of Health action framework is designed around four action areas and one outcome area. Action areas are the core areas in which investment and activity are needed: (1) making health a shared value; (2) fostering cross-sector collaboration to improve well-being; (3) creating healthier, more equitable communities; and (4) strengthening integration of health services and systems. Each action area contains a set of drivers indicating where the United States needs to accelerate change and a set of measures illustrating places for progress. Within the primary Culture of Health outcome-improved population health, well-being, and equity - the authors identified three outcome areas: enhanced individual and community well-being, managed chronic disease and reduced toxic stress, and reduced health care costs.

\section{www.rand.org}

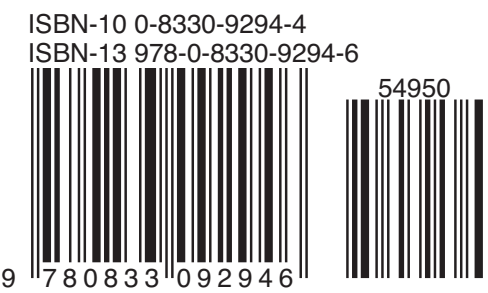

